The abstracts are only available online, free of charge, under www.karger.com/doi/10.1159/000343874

\title{
The 5th Asia-Pacific Primary Liver Cancer Expert Meeting (APPLE 2014)
}

A Bridge to a Consensus on HCC Management

Taipei, Taiwan, July 11-13, 2014

\section{Abstracts}

Guest Editors

Ann-Lii Cheng, Taipei

Chih-Hung Hsu, Taipei

Chiun Hsu, Taipei 


\section{Contents}

\section{Main Session}

Pre-Congress Meeting: How to Control HCC in Asia-Pacific Region 288

Session 1: Anti-Viral Therapy for HCC Prevention 291

Session 2: Unsolved Problems for the Treatment of HCC by TACE 294

Session 3: New Frontiers of Curative Treatment for HCC 297

Session 4: State-of-the Art Lecture: HCC Prevention: Looking into the Future 300

Session 5: Professor Juei-Low Sung Award Lecture 303

Session 6: Future Treatment for HCC 304

Session 7: Can We Find New Therapeutic Targets for HCC? 307

Session 8: Treatment of Advanced HCC: Exploring the Uncharted Territories 309

Session 9: Asian Perspectives for Pivotal Trials of HCC: Why Data Are Different? 312

Session 10: APPLE Consensus Workshop: Searching Consensus for Controversies 315

Satellite Symposium

Satellite 1: Current Evidence and Efforts to Integrate Radiotherapy into HCC Guidelines 318

Satellite 2: Biliary Tract Cancer 321

Satellite 3: Biomarkers and Drug Development 324

Satellite 4: Bridging Clinical and Histological Diagnosis of HCC 327

Satellite 5: Novel Imaging Modality 329

Poster Session

Category 1. Prevention of HCC 333

Category 2. Prediction of HCC Recurrence 338

Category 3. Surveillance and Diagnosis of Early HCC 346

Category 4. Controversial Issues in Early HCC 354

Category 5. Curative Treatment by Surgery 356

Category 6. Current Understanding of the Biology of HCC 359

Category 7. Recent Progress of Transarterial Treatment 365

Category 8. Unresolved Issues for Advanced HCC 370

Category 9. Controversies for the Locally Advanced HCC 380

Category 10. Novel Approach and Innovative Trials 384 


\section{Hosting Chairman's Note}

\section{Prof. Ann-Lii Cheng}

Department of Oncology, National Taiwan University Hospital, Taipei, Taiwan

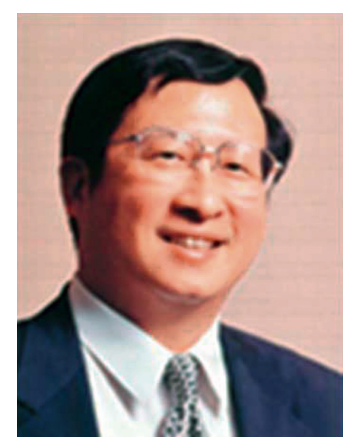

Dear Colleagues,

Thank you very much for your great support to APPLE 2014. In continuation of APPLE tradition, this year's meeting will have a comprehensive coverage on topics of diagnosis, treatment, and prevention of hepatocellular carcinoma. In addition, APPLE 2014 has the following new features:

1. The APPLE consensus development sessions. They will be divided into 5 groups. Each group will identify topics pertinent to Asian-Pacific practice for consensus development. Consensus on these topics will be derived primarily by experts' opinions and will become useful references for real-world practice and future evidence-pursuing clinical trials.

2. New topics in APPLE-sponsored satellite symposiums, including biliary tract cancers and HCC pathology.

We would like to express our most sincere gratitude to the contributions from every expert and we hope you will have an inspiring and fruitful meeting in Taipei.

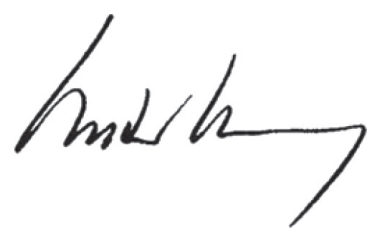




\section{Abstracts}

\section{Pre-Congress Meeting}

\section{How to Control HCC in Asia-Pacific Region}

08:30-10:00, July 11, 2014 (Auditorium)

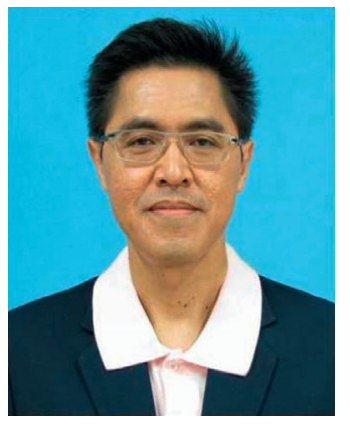

Chair

Tawesak Tanwandee

Associate Professor and Head, Division of Gastroenterology, Department of Medicine, Siriraj Hospital, Mahidol University, Bangkok, Thailand

\section{Speech Title 1}

\section{Unresolved Issues in Current Guidelines}

Diana Payawal

Cardinal Santos Medical Center, Fatima Medical University Hospital, Manila, Philippines

Dr. Diana Payawal is a graduate of University of Santo Tomas of which she took her fellowship training in Gastroenterology. She pursued further studies in Hepatology and Interventional Sonology with University of Tokyo under the MOMBUSHO scholarship. She has been the Past President of Philippine Society of Gastroenterology (2010) and the Past President of the Hepatology Society of the Philippines (2012-2014). She is now a member of the Executive Committee of the Asian Pacific Study of the Liver (APASL, 2011-present), and was the Chairperson of the Liver Cancer Single Topic Conference of the APASL 2013. Presently, she is the Chairperson of the Advocacy Committee of the said organization. She is the Chair of Section of Gastroenterology of Fatima Medical University Hospital and Asst. Chair of Section of Gastroenterology of Cardinal Santos Medical Center. She remains to be the Clinical Asst. Professor of University of Santo Tomas Hospital.

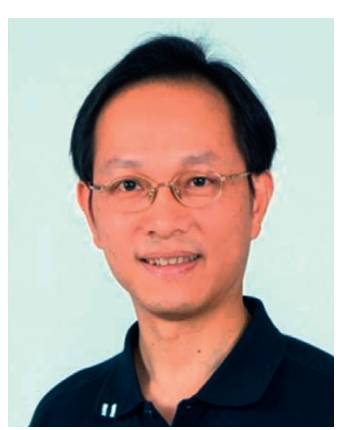

Chair

Chun-Jen Liu

Professor,

Graduate Institute of Clinical Medicine, National Taiwan University College of Medicine, Taipei, Taiwan

\section{Speech Title 2 \\ Prevention of Viral Hepatitis B in Korea Ok Park}

Division of Vaccine Preventable Diseases Control and National Immunization Program, Korea Center for Disease Control and Prevention, Seoul, Korea

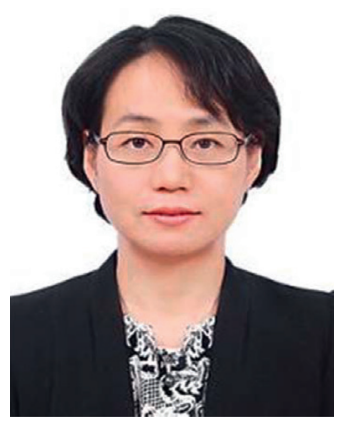

Dr. Ok Park is the director of Division of Vaccine Preventable Diseases Control and National Immunization Program in Korea Center for Disease Control and Prevention. She has worked for Korea Center for Disease Control and Prevention since 2000 in the field of communicable disease surveillance and response. She was involved in development of Electronic Reporting System of National Notifiable Diseases Surveillance System (NNDSS) and development of several sentinel surveillance systems such as Korea Influenza and Respiratory Virus Surveillance System and School Sentinel Surveillance System, etc. 
The 5th Asia-Pacific Primary Liver Cancer Expert Meeting (APPLE 2014)

\section{Abstract}

Liver cancer has posed a huge disease burden on the Korean population. Liver cancer is the 4 th most common cancer in Korea after stomach cancer, colon cancer and lung cancer in order. Chronic viral hepatitis B and C are the major causes of hepatocellular carcinoma (HCC) in Korea.

Especially, hepatitis B virus infection rate is very high and over $70 \%$ of HCC is related to hepatitis B infection. In the prevaccination era before 1980 s, $6.6 \sim 8.6 \%$ of the general Korean population tested positive for HBsAg. To reduce the high disease burden of hepatitis B, the Korean Government implemented public health programs such as universal viral hepatitis B vaccination for neonates in 1995 and vertical transmission prevention program for the neonates from HBsAg positive mothers in 2002. TheHBsAgpositiveratehas been decreasing thanks to the success of those vaccination programs. The HBsAg positive rate in adults aged over 20 years is about $4 \%$ and less than $1 \%$ in aged less than 20 years in 2012 according to the Korea National Health and Nutrition Survey data. Through vertical transmission prevention program initiated in 2002, hepatitis Bimmunoglobulin and hepatitis $B$ vaccination were provided to about 170,000 neonates and the success rate of the vertical transmission reached about $97 \%$. Also the cost for the diagnosis and treatment of acute and chronic hepatitis B is covered by the National Health Insurance.

Hepatitis C infection rate is less than $1 \%$ in individuals aged less than 50 years $-0 \%$ in aged 10 18, 0.1\% in aged $19 \sim 29,0.3 \%$ in aged $20 \sim 39,0.9 \%$ in aged $40 \sim 49-$ and $1 \sim 2 \%$ in aged older than 50 years. The cost for the diagnosis and treatment of chronic hepatitis $C$ is covered by the National Health Insurance like hepatitis B as well.

Despite of the successful implementation of the National Immunization Program, the disease burden of viral hepatitis and liver cancer is still high in Korea, various programs to reduce the disease burden should be in place in the future.

\section{Speech Title 3 \\ Issues of Under-Represented Patient Populations in Current HCC Trials}

\section{Deepak Amarapurkar}

Department of Gastroenterology and Hepatology, Bombay Hospital and Medical Research Centre, Mumbai, India

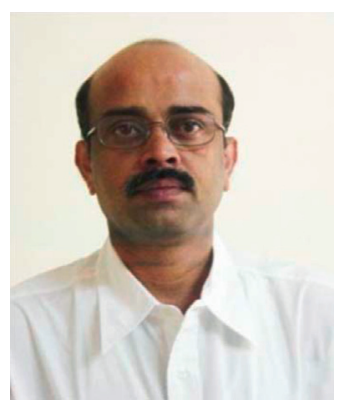

Dr. Amarapurkar is a consulting Gastroenterologist \& Hepatologist in Bombay Hospital \& Jagjivanram Western Railway Hospital since 1994. He is actively involved in various research projects; areas of interest have included Hepatitis $B$, Hepatitis C, NAFLD and HCC. He serves on the editorial boards of Journal of Gastroenterology \& Hepatology, World Journal of Gastroenterology, and Euro-Asian Journal of Gastroenterology. He has served as Treasurer, Secretary \& vice President of Indian National Association of Study of Live. He has presented 200 papers and is the author or co-author of more than 400 publications and contributed more than 20 chapters in various text books.

\section{Abstract}

Hepatocellular carcinoma (HCC) accounts for $4 \%$ of all newly diagnosed cancers worldwide. Around 6 lacs of patients are diagnosed with HCC every year. More than $80 \%$ of patients have viral infections and more than $90 \%$ of patients have liver cirrhosis. Median survival rates are $21 \%$ and $6 \%$ at the end of 1 year and 3 years respectively. $80 \%$ of the patients with HCC are in the Asian countries. Improved surveillance, diagnostic modalities and treatment options have led to the improvement in survival in patients with HCC in Japan and other developed countries. In spite of these advances care of HCC patients is suboptimal in Asian countries because surveillance practices are not followed. Treatment options in advanced HCC are limited and complicated. The prognosis and treatment eligibility depends on liver status and tumour status. Majority of the patients present with advanced HCC and hence reducing the chance of curative treatment. Treatment modalities like locoregional ablative therapy surgical resection and liver transplantation are not feasible with patients with multi-centric and recurrent disease. Trans-arterial chemoembolization and trans-arterial radio-embolization are the main stay of treatment for intermediate HCC. They are not useful in patients with advanced disease. Sorafenib a multi-kinase inhibitor with antiangiogenic and anti-proliferative properties is recommended in patients who have a performance status of zero, no constitutional symptoms and Child class A/B, by two large randomised controlled trials. Clinical trials constitute the gold standard to assess the efficacy and safety of new medicines. However, because they are conducted in standardized conditions far from the real world of prescription and use, discrepancies in patient selection or treatment conditions may alter both the effectiveness and risks. Clinical trial for Sorafenib had included mostly patients with Child Class A and good performance status. Subsequently in post marketing studies drug was found to be useful in patients with Child Class B. Still there is no appropriate treatment for all the patients with Child Class $\mathrm{C}$ and poor performance status which constitute more than $40 \%$ patients in clinical practice. Efficacy of a drug in clinical trials is assessed under highly selected motivated patients, experienced physicians and support staff and optimal conditions. Medication and evaluation tests are free. These results may not be applicable in real life scenario where all patients need treatment. Physicians 
The 5th Asia-Pacific Primary Liver Cancer Expert Meeting (APPLE 2014)

with varying knowledge and experience are treating. Monitoring of response and side effects are sub-optimal. The new medical therapies should be evaluated in patients with advance stage i.e. BCLC stage $\mathrm{C}$ and good liver function where they have proven positive impact on patients survival. Medical therapies in adjuvant settings need to be evaluated. Efficacy of medical treatment for patients with advanced HCC and less favourable disease characteristic such as portal invasion or reduced liver function i.e. Child Class C and poor performance status are not available as yet. No effective treatments are available for patients with BCLC stage D, current therapies are unlikely to offer them any benefit. Future treatment approaches need to address the patients who are underrepresented in clinical trials.

\section{Speech Title 4 Opportunities of Early Phase Clinical Trials in Asia}

Chee-Kiat Tan

Department of Gastroenterology and Hepatology, Singapore General Hospital, Singapore

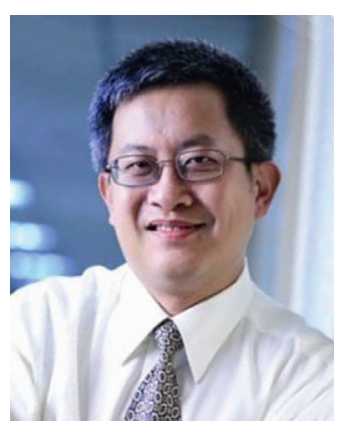

Dr. Tan is currently a senior consultant in the Department of Gastroenterology and Hepatology in the Singapore General Hospital. He is also the Deputy Director for Solid Organ Transplantation in SingHealth and was previously Director of the Liver Transplant Service in SGH from 2004-2012. He holds positions of Adjunct Associate Professor in the Duke-NUS Graduate Medical School as well as Clinical Associate Professor in the Yong Loo Lin School of Medicine, NUS. Dr. Tan sits on the Ministry of Health's Drug Advisory Committee and Transplantation Advisory Committee as well as the Medicines Advisory Committee of the Health Sciences Authority. His special interests are in liver transplantation, liver cancer and viral hepatitis and he has started a national registry on hepatocellular carcinoma. He has more than 50 publications, 2 book chapters and more than 100 abstracts presented at various regional and international meetings.

\section{Abstract}

Almost $80 \%$ of all HCCs occur in Asia. The 2 most populous countries in the world viz China and India are both in Asia and both have high rates of HCC. Hence one would expect a large number of HCC early phase clinical trials to be running in Asia.

However, this is not so. A check with ClinicalTrials.org shows that over the past 5 years, there were at most 13 phase I and 35 phase II clinical trials in Asia. This is a mere fraction compared to the 108 and 190 phase I and II clinical HCC trials worldwide over the same 5 year period. It is even more telling that in India, the second most populous country in the world, there were no phase I clinical HCC trial and only 1 phase II clinical HCC trial over the past 5 years. That phase II trial was a local trial registered with ClinicalTrials.gov.

From these data, one can surmise that the opportunities of early phase clinical trials in Asia are not being realised despite $80 \%$ of HCCs worldwide occurring in Asia. Why is this so?

One obvious reason is the lack of infrastructure in many developing nations in Asia to support the running of a clinical trial. Of all the infrastructures, perhaps the most important is communications. Opportunities to run early phase clinical trials can be realised only in the better developed Asian nations with good and efficient communications system as communication with study HQ and study leads are critical for the timely dissemination of data to ensure the safety of study subjects.

A second reason is the advanced disease stage of many patients at the time of diagnosis of HCC. In the developing Asian countries, access to medical care is not always readily available and running HCC surveillance programmes are a challenge. Hence patients often present at a late stage of disease with poor liver functional status thus rendering them ineligible for clinical trials as most HCC clinical trials require Child-Pugh A patients with good performance status.

Although it is routinely feasible to run early phase HCC clinical trials in the few developed Asian countries, it is a pity that developing nations in Asia with their large number of HCC patients are deprived of the opportunities for early phase clinical trials. It is difficult to transform a whole nation to enable it to have the opportunity to run early phase HCC clinical trials but it may be possible that attention be focused on selected high volume centres within the country to enable it to run an early phase HCC clinical trial. Keeping this in mind, there may be avenues for early phase HCC trials to flourish in Asia in the future. 


\title{
Session 1
}

\section{Anti-Viral Therapy for HCC Prevention}

\author{
10:30-12:00, July 11, 2014 (Auditorium)
}

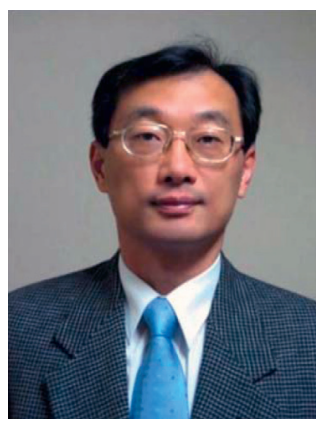

\author{
Chair \\ Kwan Sik Lee \\ Professor, \\ Yonsei University College of \\ Medicine, Seoul, Korea
}

Speech Title 1

Anti-HBV Therapy for Secondary and Tertiary Prevention of HCC

Tetsuya Hosaka

Toranomon Hospital, Tokyo, Japan

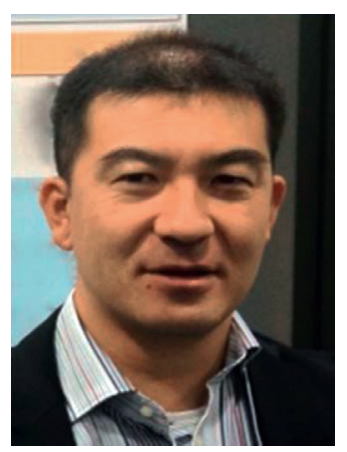

Dr. Hosaka obtained M.D. degree in 1998 from Osaka University and started training as a physician of gastroenterology and Hepatology. He has been working on the clinical research of viral hepatitis (HBV \& HCV) and hepatocellular carcinoma since he was enrolled to the Toranomon Hospital, which has the largest numbers of patients who are suffering from liver disease in Japan.

\section{Abstract}

The tumor recurrence rate after curative resection and local ablation is still high even in patients with small HCCs. Recurrences in the remnant liver can occur based on two characteristics of HCC: intrahepatic metastasis from the primary tumor and de novo multicentric carcinogenicity. Although tumor status at the first treatment is associated with recur-

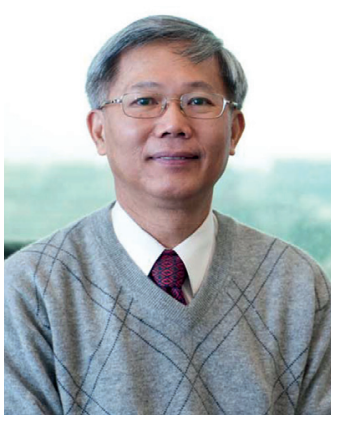

Chair

Pei-Jer Chen

Professor,

Graduate Institute of Clinical

Medicine, National Taiwan

University and Hospital, Taipei,

Taiwan rence and prognosis, the status of chronic liver disease (viral hepatitis) is also important prognostic factors. Oral nucleot(s) ide analogues (NAs) have been used as the mainstay therapeutic strategy against chronic hepatitis B. Entecavir and tenofovir which have high antiviral and low drug-resistant potency have already been available. Although patients with HBV-related cirrhosis have a significantly high risk of developing HCC, NA therapy can delay the progression of liver disease and reduce the risk of HCC in patients with cirrhosis by strong viral suppression. The benefit of adjuvant NA therapy after curative resection and local ablation is not confirmed yet because randomized control trials and largescale cohort studies which were published are few. Theoretically, NA seems to improve liver function of the remnant liver by strong viral suppressions, and subsequently reduce HCC recurrences. The recurrence rate of HBV-related HCC after curative resection is estimated to be high, and is associated with viral factors, including HBeAg positivity and the viral load before surgery, besides host and tumor factors. This presentation will review the necessity of adjuvant NA therapy for preventing recurrence. 


\section{Speech Title 2}

\section{Anti-HCV Therapy for Secondary and Tertiary} Prevention of HCC

\section{Lai Wei}

Peking University Hepatology Institute, Beijing, China; Peking University People's Hospital, Beijing, China

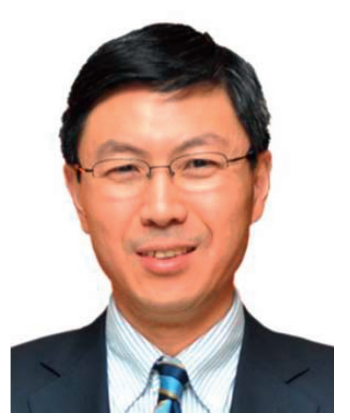

Dr. Wei is a Professor of Hepatology \& Medicine, and is the Director of Peking University Hepatology Institute since 2004 and Chief of Department of Hepatology at Peking University People's Hospital since 2001. His current research interests involve antiviral treatment of hepatitis B and C. Dr. Wei is the President of the Chinese Society of Hepatology. He served to develop the Chinese Guideline for Management of Hepatitis B, and Management, Diagnosis and Prevention of Hepatitis $C$ virus Infection. In addition, Dr. Wei is involved in Kidney Disease Improving Global Outcome to develop a Guideline for HCV Infection, Diagnosis, Prevention and Management in Kidney Disease and contributes to APASL for hepatitis $C$ guideline.

\section{Abstract}

The World Health Organization (WHO) estimates that more than 185 million people worldwide or $2.8 \%$ of the human population had been infected with HCV; of these 130-170 million are chronically infected and 350,000 deaths occur each year as a result of HCV-related cirrhosis and liver cancer. The prevalence of HCV varies from $1.2 \%$ to $3.8 \%$ in different regions of the world. HCV is recognized as the most common cause of hepatocellular carcinoma (HCC) and the most frequent indication for liver transplantation in North America, Western Europe as well as in an Asia country, Japan. In Asian HBV or HCV related cirrhotic patients without any antiviral therapy, HCC and mortality rates were significantly higher in patients with HCV than HBV-related cirrhosis.

Elevated serum levels of HCV RNA and HCV genotype 1 infection are identified as independent risk predictors of hepatocellular carcinoma in Asian, compared with non genotype-1. Genotype 3 infection was found to have a higher risk of developing cirrhosis and HCC than HCV genotype 1 patients in US.
The most effective approach to preventing HCC is to prevent HBV and HCV infection through vaccination. However, no effective prophylaxis vaccine for HCV is available up to now. Therefore, preventing of HCC is completely depended on antiviral therapy for persons chronically infected with HCV, in term of secondary and tertiary prevention of HCC development.

The regimens combining pegylated interferon and ribavirin for 24 to 48 weeks, depending on the genotype, was considered as standard of care for chronic hepatitis $\mathrm{C}$ for decade. It is well accepted that sustained virologic response (SVR), i.e. undetectable HCV RNA at 24 weeks after treatment stop, is associated with resolution of liver disease including fibrosis. Cumulated evidence indicated that antiviral therapy works well on the prevention of HCC development a few of randomized controlled trials by Pegylated interferon plus ribavirin. Meanwhile more evidence concluded that long-term virological response can improve liver histology, therefore, prevent HCC by slowing progression of liver disease and possibly even reversing liver damage. However, HCC incidence is higher in patients with cirrhosis; there still exist the risk of HCC in patients with chronic HCV infection if treatment is initiated after cirrhosis is established. Because of this, treatment at early stage might be of greater benefit. Even for cirrhotic patients, anti-HCV treatment can also reduce the risk of HCC development, although the SVR is lower compared with non-cirrhosis. Recently developed directly-acting antivirals might be tolerated for cirrhotic patients and increase SVR.

There is limited evidence for the role of anti-HCV treatment in tertiary prophylaxis of HCC in chronic Hepatitis $\mathrm{C}$ patients. Some studies suggested that the 3- and 5-year overall survival rates of patients who received peg-IFN therapy after hepatic resection were significantly higher than those of patients who did not receive IFN therapy. Metaanalysis analysis also showed that IFN-alpha treatment after curative treatment of primary tumor may be effective for the prevention of HCC recurrence, and higher SVR rate may be associated with better preventive effect. 
The 5th Asia-Pacific Primary Liver Cancer Expert Meeting (APPLE 2014)

\section{Speech Title 3 \\ Anti-Viral Therapy: Health Policy and Resource Allocation Issues}

Chun-Ying Wu

Division of Gastroenterology, Taichung Veterans

General Hospital, Taichung, Taiwan

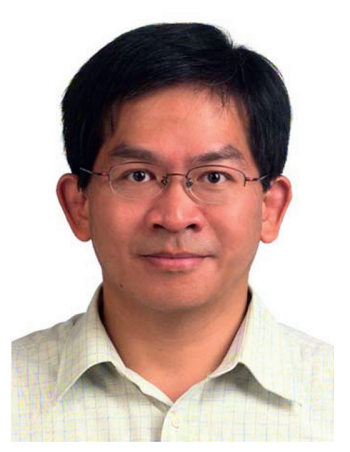

Prof. Wu has positions as Professor of Medicine at the National Yang-Ming University, the National Chung Hsing University, and the China Medical University. He is now the ViceSecretary General of both the Gastroenterological Society of Taiwan and the Digestive Endoscopy Society of Taiwan, the executive of the Taiwan Liver Cancer Association, and the control board of the Taiwan Evidence-based Medicine Association. His research interests focus on translational medicine of digestive cancers and peptic ulcer diseases. He has published many articles in top ranking peer-reviewed journals such as JAMA, J Clin Oncology, Gastroenterology, Hepatology, Gut, Clinical Cancer Research, Arthritis Rheumatology, etc. Prof. Wu is also very active in legal societies. His legal research interests include medical malpractice litigation and biotechnology regulation. He has published two law textbooks, four chapters in law books, and many articles in peer-reviewed journals including Taiwan Law Review, Internal Medical Journal, FT Law Review, Journal of New Perspective on Law, etc.

\section{Abstract}

More evidences suggest the essential roles of antiviral therapy not only in preventing hepatocellular carcinoma (HCC) development and recurrence, but also attenuating hepatic and extra-hepatic disease progressions. However, its high cost limits the widely use of antiviral therapy. The regulation of antiviral therapy has become an important healthy policy and also a resource allocation issue. In this talk, we will discuss our most updated studies regarding these issues.

Based on nationwide cohort studies, we approved the secondary and tertiary chemopreventive effects of antiviral therapy in HCC. In patients with chronic hepatitis B virus (HBV) infection, the 7-year incidences of HCC for antiviral therapy treated patients and the matched hepatoprotectants treated patients were $7.32 \%$ and $22.7 \%$, respectively. The adjusted hazard ratio (HR) for antiviral therapy was 0.37 (Gastroenterology 2014). For HBV-related HCC after liver resection, antiviral therapy use is associated with lower risks of HCC recurrence and mortality. The treated cohort had significantly lower 6-year HCC recurrence rate (untreated vs. treated: $54.6 \%$ vs. $45.6 \%$ ) and overall mortality (untreated vs. treated: $42.4 \%$ vs. $29.0 \%$ ), respectively. Antiviral therapy was independently associated with a reduced risk of HCC recurrence (HR = 0.67) (JAMA 2012). For those with chronic hepatitis $\mathrm{C}$ virus (HCV) infection after liver resection, the 5-year HCC recurrence rate was significantly lower in the antiviral therapy treated cohort (52.1\%) than untreated cohort (63.9\%). The adjusted HR was 0.64 for antiviral therapy (Hepatology 2013).

In addition to HCC chemoprevention, antiviral therapy also attenuates hepatic and extra-hepatic disease progressions. Based on population-based cohort studies, we found antiviral therapy in chronic HBV-infected patients was associated with a significantly lower risk of liver decompensation, liver failure and overall mortality. For those infected with chronic HCV, antiviral therapy was associated with reduced 8-year cumulative incidences of ESRD (treated vs. untreated: $1.1 \%$ vs. $9.3 \%$ ), ischemic stroke (treated vs. untreated: $3.1 \%$ vs. $5.3 \%)$, and acute coronary syndrome ( $4.1 \%$ vs. $6.6 \%)$, respectively. The adjusted HRs of antiviral therapy were 0.16 for ESRD, 0.53 for ischemic stroke, and 0.64 for acute coronary syndrome, respectively (Hepatology 2014).

How to integrate these important evidences found in the real world into health policies and allocate health care resources are important issues. More studies to identify those who benefit most and to find the cost-benefit health care practices will be needed for regulating antiviral therapy. 


\title{
Session 2
}

\section{Unsolved Problems for the Treatment of HCC by TACE}

\author{
13:45-14:55, July 11, 2014 (Auditorium)
}

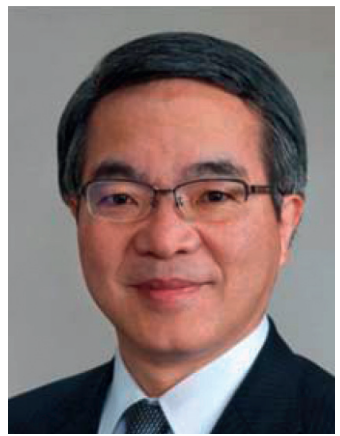

Chair

Namiki Izumi

Vice President,

Musashino Red-Cross Hospital,

Tokyo, Japan

\section{Speech Title 1 \\ Defining the Boundary between Surgery and TACE}

Thomas Yau

Department of Clinical Oncology, Li Ka Shing Faculty of Medicine, The University of Hong Kong, Hong Kong

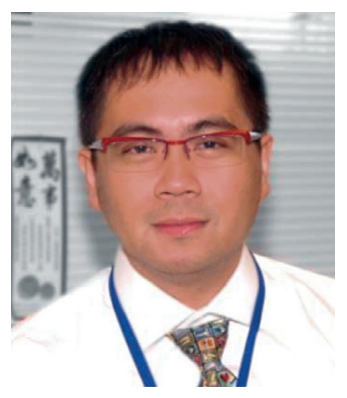

Dr. Yau is currently a clinical assistant professor at the University of Hong Kong. His main research interests are gastrointestinal oncology, early phase clinical trials and translational research. He had published more than 90 peer-review publications with articles in various leading oncology journals, namely Cell Stem Cell, Lancet Oncology, Gastroenterology, Hepatology, Clinical Cancer Research, Oncologist and Cancer. He currently serves as editor-in-chief, associate editor and the editorial board members of many international peer-reviewed journals. Dr. Yau is currently the Vice President of Asia Pacific Neuroendocrine Tumour Society, international Faculty Board Member of International Association of Surgeons Gastroenterologist and Oncologists, General Secretary of Hong Kong Liver Cancer Foundation, and member of many international professional societies.

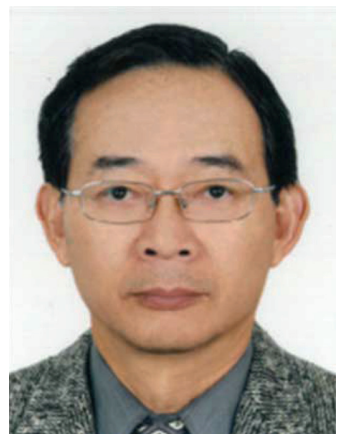

Chair

Chung-Kwe Wang

Deputy Superintendent,

Taipei City Hospital Renai Branch,

Taipei, Taiwan

\section{Abstract}

One of the controversies issues in the management of patients with hepatocellular carcinoma is to define the clear boundary between surgery and TACE. BCLC treatment algorithm was aimed to provide the treatment algorithm for HCC patients worldwide. Nevertheless, it is mostly used in Europe and North America but not yet in Asia due to the differences in the clinical management of HCC. Notably, the simplicity of the BCLC classification came at a price, in particular compromising its usefulness in stratifying patients to receive either surgical or loco-regional therapies. Recently, our group developed a new prognostic staging system (HKLC) with treatment guidelines based on a large HCC cohort in Hong Kong. Overall, our HKLC treatment algorithm yielded better survival outcomes when compared with the BCLC treatment algorithm. Our proposed HKLC treatment guidelines expand the criteria for potential curative therapies to include patients with large or multiple tumors, and even with intrahepatic venous invasion. Our results showed that hepatic resection in a properly selected advanced HCC subgroup of these patients might still derive substantial survival benefits. Moreover, TACE might also achieve survival benefits in some of the BCLC-C patients. 
The 5th Asia-Pacific Primary Liver Cancer Expert Meeting (APPLE 2014)

\section{Speech Title 2 \\ Drug Eluting Microspheres: Basic Principles and Evidence}

David Liu

University of British Columbia, Vancouver, Canada

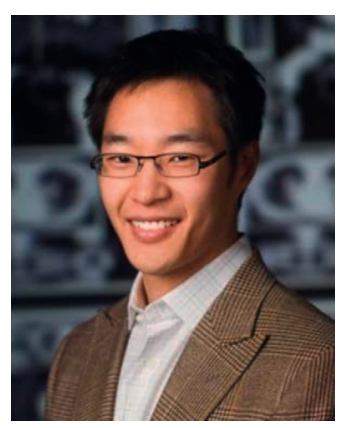

Dr. Liu is a Clinical Associate Professor of Radiology at the University of British Columbia. He maintains an active interventional oncology practice incorporating all aspects of embolization, ablation, venous access, and palliative therapy in all organ systems. Dr. Liu is the co-founder and co-chair of SHOW, and has been credited with 4 book chapters, over 40 publications, and 70 invited lectures, and is an active member and inducted fellow of the Society of Interventional Radiology. He holds specific interest in the treatment of hepatocellular carcinoma and metastatic colorectal carcinoma. Dr. Liu is actively involved in both translational research and clinical trials.

\section{Abstract \\ Concept and Theoretical Benefits}

The rapid adaptation of drug-eluting microparticle platforms is based on the premise that the synergistic actions of the physical particle acting as an embolic device, married with the ability to provide sustained compartmentalized drug exposure within the region of particle delivery results in optimized locoregional tumoricidal effect, and as a result, improvement in systemic toxicity, local tumor kill, and ultimately improvement in overall survival.

\section{Embolic}

Controversy remains regarding the optimal use and progression of particle amount and size. Ultimately, it appears that the ideal particle size should be optimized for the embolic effect (i.e. terminal embolization as an endpoint) with carrier based delivery of drug functioning as a supplement, providing 'collateral coverage' in the arena of hypervascular lesions such as HCC and NET. The profound embolic effect of super absorbant microspheres (SAP-MS) appear to result in improved histological, and radiographic response, suggesting more effective tumoricidal effect. Optimal size for application in less vascular tumors (in addition to optimal drug) has remained elusive for $\mathrm{mCRC}$ and cholangiocarcinoma with a trend towards carrier based delivery as opposed to embolic coverage.

\section{Carrier}

Current drug eluting platforms avail the ability to load and elute chemotherapeutic via the inherent difference in the ionic milieu at the time of loading, as compared to the targeted parenchyma/tumor. This phenomenon manifests as the ability to load ionically charged molecules of therapeutic onto oppositely charged moieties on microsphere surface in a controlled environment (i.e. pharmacy fume hood). The loaded particle is then delivered to the target organ, where over time, osmosis, ionic exchange, changes in the $\mathrm{pH}$, particle deterioration, and perhaps the acute inflammatory response result in predictable, sustained release of drug (with a varying proportion imbedding permanently, and irreversibly to the particle matrix).

Exposed surface area of the particle is a significant factor in loading capacity and elution. The surface area of the particle is inversely proportional to the size of the particle by the following relationship:

\section{Surface Area $=4 \pi r^{2}$}

Thus, a change in mean particle size from 600 to $200 \mathrm{um}$ will result in an increase in surface area (assuming same mass or volume) of 9 fold, resulting in a significantly faster loading and elution.

\section{Chemotherapies (Disease state)}

As previously outlined, the ability to load drug molecule into drug-eluting platform is dependent on the ionic interaction between particle and drug molecule. Particle size (ie surface area), polarity and charge of the therapeutic molecule (collectively referred to as the Zeta) play a significant role in the loading capacity and elution rates of drug.

Anthracycline class drugs, commonly used in the treatment of HCC demonstrate high polarity and as a result load rapidly and at higher concentration, with slower elution times (days to weeks). Other drugs such as CPT-11 (irinotecan) demonstrates very low Zeta and as a result demonstrates very low overall loading capacity and very rapid elution. (minutes to hours)

In comparison, completely neutral molecules, such as platinum class chemotherapeutics (e.g., cisplatin) may only load and elute due to non-ionic interaction with particle matrix, of which the primary mechanism of drug retention and delivery is unknown.

Anthracycline class chemotherapeutics such as adriamycin and epirubicin demonstrate direct effects on tumoral cells, however fundamental mechanisms of action remain unknown but have demonstrated efficacy in the treatment of HCC. Adriamycin has not been proven to be effective in the treatment of mCRC either as a systemic or locoregionally delivered drug. Interestingly, irinotecan itself is not active, and its metabolite, $\mathrm{SN}-38$ serves as the active metabolite (and is only metabolized in normal liver, not tumor). Thus, the true effect of DEBIRI is likely activation of the loaded irinotecan as a result of accumulation of particles along the tumor/liver 
The 5th Asia-Pacific Primary Liver Cancer Expert Meeting (APPLE 2014)

interface, first pass extraction after systemic circulation (pk analysis makes this highly unlikely), or as a result of the nontargeted embolization of particles in the liver parenchyma.

\section{Summary}

The use of statically charged drug eulting matrices in embolics represent a different class of embolotherapy with many factors effecting the potential for optimal therapy and response. Decreased toxicity has been demonstrated with elution platforms, primarily due to decreased systemic exposure, with a trend towards improvement in radiographic response with a more embolic component in HCC and NET. The user should be cognizant of the biology of the targeted cancer, type of chemotherapeutic, the size of the particle and the potential for irreversible sequestration of drug when determining the ideal approach, for current 'standardized' approaches to therapy remain elusive based on the relative lack of data outside of the HCC domain.

\section{Speech Title 3 \\ Expert Panel Opinion for TACE in HCC: The EPOIHCC View}

\section{Seung Woon Paik}

Division of Gastroenterology, Department of Medicine, Samsung Medical Center, Sungkyunkwan University School of Medicine, Seoul, Korea

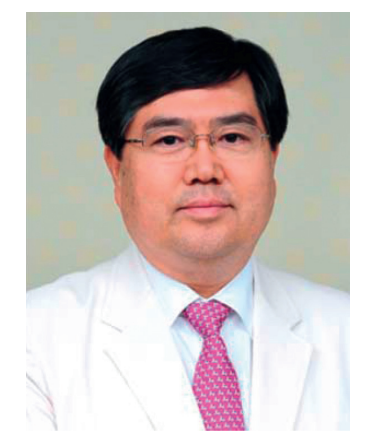

Prof. Paik is currently a Professor in the Department of Medicine/Gastroenterology at Samsung Medical Center and a Director of Liver Cancer Center at Samsung Medical Center in Seoul, Korea. He served as a Secretary General of the Korean Association of the Study of the Liver during 2010-2011. In 2011, he hold APASL single topic conference successfully in Jeju as a Secretary General of the organizing committee. Currently he serves as a President of the Korean Liver Cancer Study Group since July of 2013. Prof Paik has participated in several global phase II and III clinical trials for hepatitis and hepatocellular carcinoma and has published many international scientific papers in well-regarded journals.

\section{Abstract}

Patients with unresectable hepatocellular carcinoma (HCC) usually receive transarterial chemoembolization (TACE) or systemic therapies with intermediate and advancedstage disease. However, intermediate-stage HCC patients often have unsatisfactory clinical outcomes with repeated TACE and there is considerable uncertainty surrounding the criteria for repeating or stopping TACE treatment. In July 2012, an Expert Panel Opinion on Interventions in Hepatocellular Carcinoma (EPOIHCC) was re-convened in Shanghai in an attempt to provide a consensus on the practice of TACE, particularly in regard to evaluating TACE 'failure'. To that end, current clinical practice throughout Asia was reviewed in detail including safety and efficacy data on TACE alone as well as in combination with targeted systemic therapies for intermediate HCC. This review summarizes the evidence discussed at the meeting and provides expert recommendations regarding the use of TACE for unresectable intermediatestage HCC. A key consensus of the Expert Panel was that the current definitions of TACE failure are not useful in differentiating between situations where TACE is no longer effective in controlling disease locally vs. systemically. By redefining these concepts, it may be possible to provide a clearer indication of when TACE should be repeated and more importantly, when TACE should be discontinued (Liver Int. 2014 Feb;34(2):174-183). 


\title{
Session 3
}

\section{New Frontiers of Curative Treatment for HCC}

\author{
15:15-16:25, July 11, 2014 (Auditorium)
}

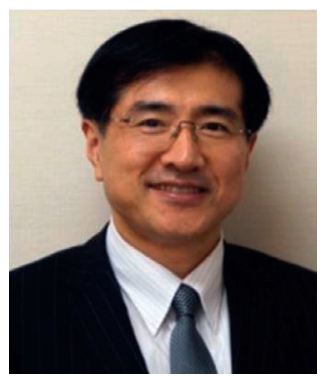

\author{
Chair \\ Norihiro Kokudo \\ Professor and Chairman, \\ Hepato-Biliary-Pancreatic \\ Surgery Division and Artificial \\ Organ and Transplantation \\ Division, Department of Surgery, \\ University of Tokyo Hospital, \\ Tokyo, Japan
}

Speech Title 1

\section{Fluorescent Image-Guided Liver Surgery}

Norihiro Kokudo

Hepato-Biliary-Pancreatic Surgery Division and Artificial Organ and Transplantation Division, Department of

Surgery, University of Tokyo Hospital, Tokyo, Japan

Dr. Norihiro Kokudo is the professor and chairman at Hepato-Biliary-Pancreatic Surgery Division and Artificial Organ and Transplantation Division, Department of Surgery, University of Tokyo. His research interest focuses on surgical treatment of HCC, colorectal liver metastases, and living donor liver transplantation. Prof. Kokudo is currently the President of Japan Surgical Society and the president elect for Asian-Pacific Hepato-Pancreato-Biliary Association (A-PHPBA). He oversaw the compilation of the 3rd version of Japanese clinical practice guidelines for HCC in 2013. He is an associate editor of Hepatology Research and Liver Cancer, and on the editorial board of many scientific journals, including Annals of Surgery and World Journal of Surgery.

\section{Abstract}

Indocyanine green (ICG) is a water-soluble, anionic tricarbocynanine dye, and is frequently used to evaluate liver functional reserve. Intra-venously administered ICG is taken into the liver, and exclusively excreted into the bile, but a portion of the injected ICG is retained in the liver tumors for about two weeks; in addition, protein-bound ICG emits light $(830 \mathrm{~nm})$ when illuminated with near-infrared light. Therefore, ICG fluorescent imaging can be used for the identification of HCC as well as liver segments during liver surgery.

For cancer imaging, ICG that was administered intravenously at the dose of $0.5 \mathrm{mg} / \mathrm{kg}$ for preoperative liver function

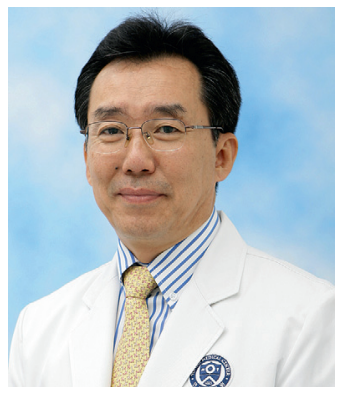

Chair

Kwang-Hyub Han

Professor and Chairman, Internal Medicine, Yonsei University College of Medicine, Seoul, Korea testing can be utilized as a fluorescence source. Intraoperatively, fluorescence images of HCC are visualized on the liver surfaces by using commercially-available fluorescence imaging system. According to our updated data, 273 nodules out of 276 HCC lesions (98.9\%) were visualized by ICG fluorescence and fluorescence pattern collated well with pathological differentiation.

For ICG guided anatomic resection, only 1/50 vial of ICG injected into the corresponding portal branch together with indigocarmine was used for the identification of the liver segment. Staining with indigocarmine only, a conventional method, sometimes fails in visualizing the border of liver segments, especially in cirrhotic livers or in cases of liver tumors compressing the portal branch. The ICG fluorescent imaging is very sensitive and identification of segmental border is possible in almost all cases. Injected ICG inside the liver parenchyma is also visible during parenchymal transection so that intersegmental plane can be identified three dimensionally. 
The 5th Asia-Pacific Primary Liver Cancer Expert Meeting (APPLE 2014)

\section{Speech Title 2 Novel Techniques: Ablation}

Shuichiro Shïna

Department of Gastroenterology, Juntendo University, Tokyo, Japan

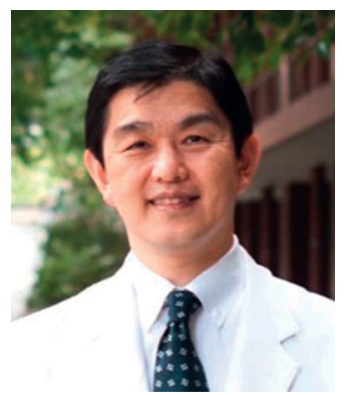

Prof. Shiina has been a pioneer of image-guided percutaneous ablation therapies for liver tumors, such as percutaneous ethanol injection, percutaneous microwave ablation, and radiofrequency ablation. His research interests are "Interventional Oncology, such as Radiofrequency Ablation", "Diagnosis and Treatment of Liver Neoplasms", and "Chemotherapy of GI tract and Liver Cancers". He published over 200 International scientific papers in high quality English Journal and over 300 domestic scientific papers. He is a member of The American Gastroenterological Association, Asian Pacific Association for the Study of the Liver, The International Liver Cancer Association, The Japanese Society of Gastroenterology, The Japan Society of Hepatology, The Japan Society of Ultrasonics in Medicine, The Liver Cancer Study Group of Japan, Study Group of Microwave Surgery, The Japan Society of Clinical Oncology, The Japan Pancreas Society, and Japanese Society for Gastroenterological Carcinogenesis and others.

\section{Abstract}

Only 20 percent of HCC patients are candidates for resection. Furthermore, recurrence is frequent even after apparently curative resection. Liver transplantation is restricted by organ donor shortage.

Thus, various nonsurgical therapies have been introduced. Among these, image-guided percutaneous ablation is considered best for early-stage HCC. Ablation has been widely performed on patients with HCC, generally for those with Child-Pugh class A or B liver dysfunction who have three or fewer tumors each $3 \mathrm{~cm}$ or less in diameter.

Ethanol injection was formerly the standard procedure among the various percutaneous ablation techniques. Our 20-year outcomes showed that ethanol injection was potentially curative for HCC, resulting in survival for more than 20 years. With a median follow-up of 51.6 months, 5-, 10- and 20 -year survival rates were $49.0 \%, 17.9 \%$, and $7.2 \%$, respectively (Shiina S, et al. Liver Int 2012).
Randomized controlled trials, however, have demonstrated that radiofrequency ablation (RFA) is superior to ethanol injection. RFA had a $46 \%$ smaller risk of death (adjusted relative risk, 0.54 [95\% CI: 0.33-0.89], $\mathrm{P}=0.02$ ), and an $88 \%$ smaller risk of local tumor progression (relative risk, 0.12 [95\% CI: 0.03-0.55], P $=0.006$ ) than ethanol injection (Shiina S, et al. Gastroenterology 2005).

In our 10-year experience of RFA, final CT showed complete tumor ablation in 2,964 (99.4\%) of 2,982 treatments performed for the 1,170 primary HCC patients. With a median follow-up of 38.2 months, 5- and 10-year survival rates were $60.2 \%$ (95\% CI $56.7 \%$ to $63.9 \%$ ) and $27.3 \%$ (95\% CI $21.5 \%$ to $34.7 \%$ ), respectively. Multivariate analysis demonstrated that age, anti-HCV, Child-Pugh class, tumor size, tumor number, serum des-gamma-carboxy-prothrombin (DCP) level, and serum lectin-reactive alpha-fetoprotein level (AFP-L3) were significantly related to survival. Five- and 10-year local tumor progression rates were both 3.2\% (95\% CI $2.1 \%$ to $4.3 \%$ ). Serum DCP level alone was significantly related to local tumor progression. Five- and 10-year distant recurrence rates were $74.8 \%$ (95\% CI $71.8 \%$ to $77.8 \%$ ) and $80.8 \%$ (95\% CI $77.4 \%$ to $84.3 \%$ ), respectively. Anti-HCV, Child-Pugh class, platelet count, tumor size, tumor number, serum AFP level, and serum DCP level were significantly related to distant recurrence (Shiina S, et al. Am J Gastroenterol 2012).

There is still an argument over which is superior in the treatment of HCC, ablation or resection. Nowadays, however, most patients prefer ablation to hepatic resection in Japan. Refined ablation is potentially curative, minimally invasive and easily repeatable for recurrence. Sophisticated ablation might be not an alternative but a superior to surgery in the treatment of small- and middle-sized HCC. 
The 5th Asia-Pacific Primary Liver Cancer Expert Meeting (APPLE 2014)

\section{Speech Title 3 \\ Surgery vs. Ablation: A New Balance in the Era of New Technologies?}

Shi-Ming Lin

Division of Hepatology, Chang Gung Memorial

Hospital, Linkuo and Taipei, Taiwan

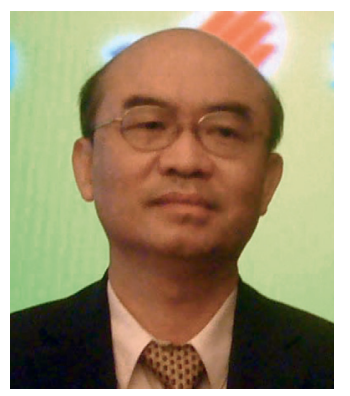

Prof. Lin is one of the pioneers of RFA for liver tumors in Taiwan; His research interest focused on antiviral treatment for hepatitis B, local ablation for HCC and tertiary prevention of HCC recurrence. Prof. Lin has published 96 papers in international and domestic scientific journals including Gastroenterology Hepatology, Gut, Journal of Hepatology, Cancer, and British Journal of Surgery. He has 4 papers (published in Hepatology 1999 and Gastroenterology 2004, Gut 2005 and Journal of Hepatology 2007) ranked as the highly cited papers. Prof. Lin has given 103 invited lectures in the area of his expertise on occasions to international audiences including APASL (2006, 2008-9, 2012, 2013), APDW (2007, 09), Annual Microwave Surg Meeting Jpn (2008, 09), Kobe HCC symposium (2009), APPLE (2010-2013), 2013 Korea-Japan Image-guide Tumor Ablation Meeting, Japan JSH 2012, HK IDD 2011, HK Interventional Radiology Symposium 2009, South East Asian Nations conference (2008, 2010, 2012), and Singapore GI Annual Meeting 2009, Asian Congress of Tumor Ablation (ACTA) 2014, and 4th International Kyoto Liver Cancer Symposium 2014.

\section{Abstract}

Resection, liver transplantation and local ablation remain the three curative treatments for very early stage (BCLC 0) and early stage (BCLC A) HCC. In general, resection is the major choice of treatment for resectable HCC without cirrhosis. However, ablation can achieve long-term survival in selected patients with resectable HCC, particularly for BCLC stage 0 and A. Some RCTs and comparative studies with propensity score matching have shown a comparative 3 to 5 -year survival after resection and conventional RFA but an equal or slight higher recurrence rate after RFA. Although recurrence-free survival after RFA is slightly lower, easy repeatability of RFA can achieve comparable long-term survival. Moreover, RFA with tumor non-touching ablation method for HCC $<3-4 \mathrm{~cm}$ by multi-electrodes are being applicable. Comparable overall survival and recurrence-free survival rate can also be anticipated.

Among RFA, conventional RFA with sequential overlapping ablation with single RF electrode has some limitations and show suboptimal results in $\mathrm{HCC}>3 \mathrm{~cm}$ and at peri-vascular location. To overcome these limitations, numerous novel ablations are undergoing in practice, including RFA with multielectrodes and switching RF controller, microwave ablation (MWA), and irreversible electroporation. More recently, some preliminary data have shown a better tumor ablation rate in early or intermediate stage HCC (3-7 cm) following novel RFA or MWA with 3-6 electrodes or antennas as compared with conventional RFA or MWA. Therefore, after longer follow-up (median 3 years), 5-year survival and recurrence rate might be improved after novel ablations. Therefore, whether patients at BCLC A and selected BCLC B can be offered local ablation or resection as a first line treatment option is a topic of controversy, particularly in patients with compensated liver cirrhosis and borderline portal hypertension. No RCT has been conducted to compare resection and novel RFA with multi-electrodes or MWA with multi-antennas in patients with BCLC stage A with multiple tumors or BCLC stage B with $\mathrm{HCC}<7 \mathrm{~cm}$ so far.

\section{References}

Seror 0, et al: Large ( $\geqq 5.0-\mathrm{cm})$ HCCs: multipolar RF ablation with three internally cooled bipolar electrodes - initial experience in 26 patients. Radiolog 2008;248:288-296.

2. Lee J, et al: Percutaneous radiofrequency ablation with multiple electrodes for medium-sized hepatocellular carcinomas. Korean J Radiol 2012;13:34-43.

3. Feng K, et al: A randomized controlled trial of radiofrequency ablation and surgical resection in the treatment of small hepatocellular carcinoma. J Hepatol 2012;57:794802.

Hung HH, et al: Survival rates are comparable after radiofrequency ablation or surgery in patients with small hepatocellular carcinomas. Clin Gastroenterol Hepatol 2011;9:79-86.

5. Wang JH, et al: Survival comparison between surgical resection and radiofrequency ablation for patients in BCLC very early/early stage hepatocellular carcinoma. J Hepatol 2012;56:412-418.

76 . Lin SM: Ultrasonography-guided radiofrequency ablation in hepatocellular carcinoma: current status and future prospects. J Med Ultrasound 2013;21:9-15.

Lin SM: Local ablation for hepatocellular carcinoma in Taiwan. Liver Cancer 2013;2:73-83. 


\title{
Session 4
}

\section{State-of-the Art Lecture: HCC Prevention: Looking into the Future}

\author{
08:00-09:15, July 12, 2014 (Auditorium)
}

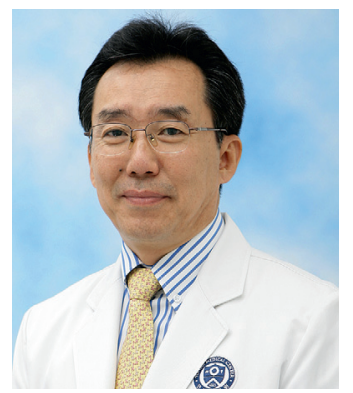

Chair

\section{Kwang-Hyub Han}

Professor and Chairman,

Internal Medicine, Yonsei

University College of Medicine,

Seoul, Korea

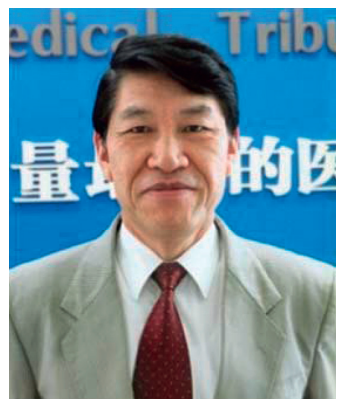

Chair

\section{Sheng-Long Ye}

Professor,

Liver Cancer Institute, Zhongshan

Hospital, Fudan University,

Shanghai, China

\section{Speech Title 1 \\ The WHO Framework for Global Actions on Viral Hepatitis: from Asia to the World}

Ding-Shinn Chen

National Taiwan University College of Medicine, Taipei, Taiwan; Academia Sinica, Taiwan

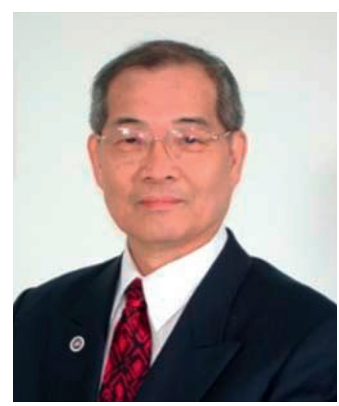

Prof. Chen is currently a Distinguished Chair Professor, National Taiwan University College of Medicine and a Distinguished Visiting Fellow, Academia Sinica. Prof. Chen's research encompasses both basic and clinical aspects of viral hepatitis and liver disease, covering from prevention to management. He

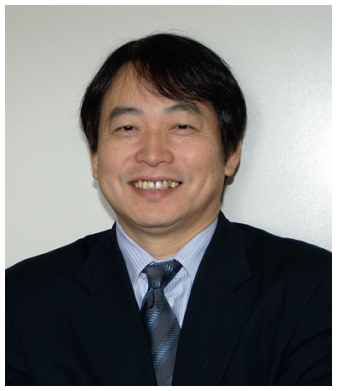

Chair

Masatoshi Kudo

Professor and Chairman,

Department of Gastroenterology and Hepatology, Kinki University School of Medicine, Osaka, Japan has a key role in the National Hepatitis B Mass Immunization Program in Taiwan, which successfully reduces the HBV infection rate and childhood HCC in Taiwan and is now one of the most successful examples of cancer prevention in the world. Prof. Chen has received numerous awards for his scientific achievement, including Academician, Academia Sinica (Taiwan); Fellow, Third World Academy of Sciences; Foreign Associate, US National Academy of Sciences; Caring Physicians of the World, World Medical Association; International Recognition Award, European Association for the Study of the Liver; Okuda Lectureship Award, Asian Pacific Association for the Study of the Liver; Distinguished Clinician/Mentor Award, American Association for the Study of Liver Diseases.

\section{Abstract}

Introduction: Worldwide, at least one third of the population have been infected by hepatitis B or C viruses, and 500 million people are chronically infected. Each year, about one million people died from viral hepatitis-related diseases, $60 \%$ of hepatic cirrhosis and $80 \%$ of hepatocellular carcinoma (HCC) were attributed to hepatitis B or C infections.

Notably, Asia Pacific carries the major part of the global burden of viral hepatitis. An estimated 74\% of all people with 
The 5th Asia-Pacific Primary Liver Cancer Expert Meeting (APPLE 2014)

hepatitis B live in South-East Asia (100 million) and Western Pacific (160 million). $20 \%$ of the total hepatitis $\mathrm{C}$ population live in the region. In mortality, $70 \%$ of deaths relating to viral hepatitis occurred in Asia Pacific (1,012,873/1,444,554), and this was more than those died of tuberculosis $(827,567), \mathrm{HIV} /$ AIDS $(304,628)$ and malaria $(106,729)$.

Methods: Because of the serious disease burden, several countries have taken actions on the control of viral hepatitis, and Taiwan was one of the earliest in forming a national program. Dating back to $>30$ years ago, in the beginning of 1980s, the government implemented a hepatitis control program to curb viral hepatitis which had been rampant in Taiwan. Three aspects were focused in the program, namely, research, public health measures and public education. Among the numerous activities implemented, mass vaccination of hepatitis B turned out to be most prominent. In 1984, we started to immunize neonates of hepatitis B surface antigen (HBsAg) carrier mothers and the immunization was extended to all neonates since 1986 . The coverage rate was $>97 \%$. The results were excellent. After the mass vaccination, HBsAg carrier rate decreased from $\sim 10-15 \%$ to $<1 \%$. More importantly, in the vaccinated cohort, the sequelae caused by HBV infection also decreased drastically including fulminant hepatitis B, membranous nephropathy, chronic liver disease (CLD) and HCC. Most recent results showed the mortality rate of CLD decreased from 0.65 per 105 of those born in 1977-80 to 0.02 per 105 of those born in 2001-04, and for HCC, from 0.83 to 0.05 per 105 persons. The decrease of HCC after hepatitis B vaccination has also been observed in China, Thailand, Japan, Korea and Alaska.

Results: Despite the success shown above, many challenges remain to be solved. Although HBV vaccines have been available since 1982, worldwide, $21 \%$ of infants still had not covered by 3 doses of the vaccine. Apparently, awareness of viral hepatitis is inadequate both in the public and the governments. In the last decade, efforts from nongovernmental organizations have also been initiated. Those from Global Alliance on Vaccines and Immunization (GAVI), World Hepatitis Alliance (WHA) and Coalition to Eradicate Viral Hepatitis in Asia Pacific (CEVHAP) are excellent examples. Most importantly, the World Health Organization (WHO) recently put forward four strategic axes for policy development in the prevention and control of viral hepatitis in 2012. The Framework for Global Action consists of 1). Raising awareness, promoting partnerships and mobilizing resources, 2). Evidence-based policy and data for action, 3). Prevention of transmission, and 4). Screening, care and treatment.

Conclusion(s): With all these efforts, we hope that viral hepatitis can be controlled as soon as possible, so that the sequelae caused by chronic viral hepatitis can be reduced as much as possible in the near future.

\section{Speech Title 2 Gut Microbiota and HCC}

Robert F. Schwabe

The Columbia University, New York, USA

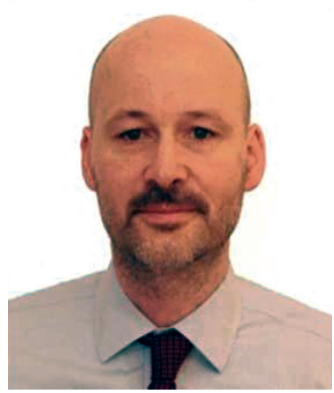

Prof. Schwabe is now an Associate Professor at the Columbia University. He is an Associate Editor for Gastroenterology (since 2011) and Journal of Hepatology (since 2009). He has received Harold and Golden Lamport Award for Excellence in Clinical Science Research (2012) and the AGA Research Scholar Award (2003-2006)

Prof. Schwabe's research focuses on mechanisms regulating liver injury, inflammation, fibrogenesis and carcinogenesis. Key areas of interest include (1) the interactions between different cell types in regulating hepatic wound healing; (2) the functional links between liver fibrosis and HCC development; and (3) the roles of the gut-liver axis in promoting liver fibrosis and HCC. His research has been published in prestigious journals including Nature Medicine, Cancer Cell, Gastroenterology, etc.

\section{Abstract}

More than $80 \%$ of hepatocellular carcinomas (HCC) develop in the setting of liver fibrosis, cirrhosis and chronic inflammation. Increased bacterial translocation is one of the hallmarks of chronic liver disease, causing many of the many infectious complications of end-stage liver disease. Moreover, increased translocation of bacteria and inflammatory bacterial products, termed pathogen-associated molecular patterns (PAMPs), such as LPS contribute to chronic liver inflammation and fibrogenesis. Here we will discuss data from murine HCC models providing strong evidence that increased bacterial translocation plays an essential role in murine hepatocarcinogenesis, and discuss clinical implications. To test the hypothesis that increased bacterial translocation is causatively involved in hepatocarcinogenesis, we employed four complementary approaches in murine HCC models: (i) Hepatocarcinogenesis was assessed in gut-sterilized mice in which the intestinal bacterial microbiota was depleted using a quadruple antibiotics cocktail; (ii) hepatocarcinogenesis was determined in germ-free mice; (iii) hepatocarcinogenesis was tested in mice that expressed non-functional TLR4, the receptor for LPS; (iv) hepatocarcinogenesis was tested in mice 
that received chronic LPS treatment via subcutaneous pumps. All four approaches demonstrated a significant contribution of the gut microbiota-LPS-TLR4 axis in hepatocarcinogenesis, with TLR4 ablation, germ-free status and gut sterilization reducing HCC development by about $80 \%$ and LPS treatment increasing HCC development. Mechanistically, the gut microbiota-LPS-TLR4 pathway prevented cell death in the malignant liver, and promoted the expression of growth factors such as epiregulin and hepatocyte growth factor (HGF). Surprisingly, HCC prevention was most efficient when antibiotics where given in late stages of hepatocarcinogenesis, suggesting that for HCC prevention by antibiotics may be considered for patients with end-stage liver disease. To further determine potential clinical applications, we tested whether Rifaximin, a well-tolerated antibiotic that is widely used in patients with chronic liver disease, may also prevent HCC. Although effects of Rifaximin were weaker than complete gut sterilization by quadruple antibiotics, it reduced HCC development by $50 \%$ when given in late stages of hepatocarcinogenesis. These results suggest that targeting the microbiota, e.g. by treatment with non-absorbable antibiotics such as Rifaximin, may be useful for prevention of HCC in patients with advanced liver disease. Implications for human hepatocarcinogenesis and strategies for clinical studies will be discussed. 


\title{
Session 5
}

\section{Professor Juei-Low Sung Award Lecture}

\author{
09:15-09:40, July 12, 2014 (Auditorium)
}

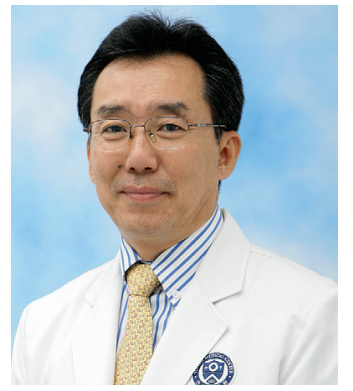

Chair

\section{Kwang-Hyub Han}

Professor and Chairman, Internal Medicine, Yonsei University College of Medicine, Seoul, Korea

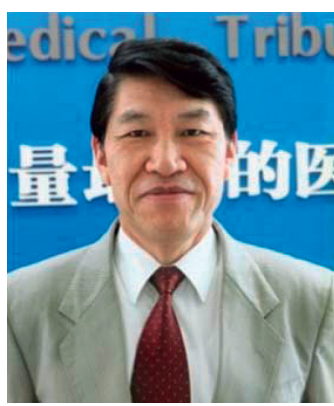

Chair

\section{Sheng-Long Ye}

Professor,

Liver Cancer Institute, Zhongshan Hospital, Fudan University, Shanghai, China

Speech Title

Predictive, Personalized and Preventive Medicine of Hepatocellular Carcinoma: Implications of REVEAL-HBV/HCV Study

Chien-Jen Chen

Academia Sinica, Taipei, Taiwan

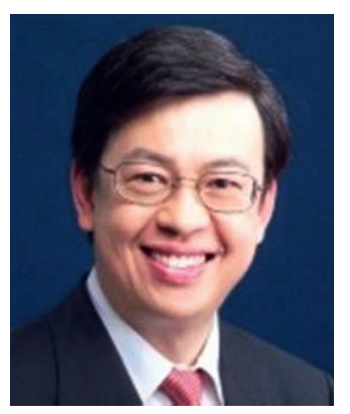

Prof. Chen is an internationally well-known epidemiologist and public health practitioner, whose pioneering researches on the natural history, disease progression, and risk estimation of end-stage liver diseases of chronic hepatitis B have been used to prevent hepatocellular carcinoma and deaths of millions of people worldwide. Prof. Chen leads the R.E.V.E.A.L.-HBV study,

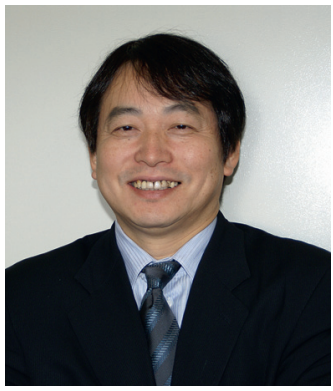

Chair

Masatoshi Kudo

Professor and Chairman,

Department of Gastroenterology and Hepatology, Kinki University School of Medicine, Osaka, Japan

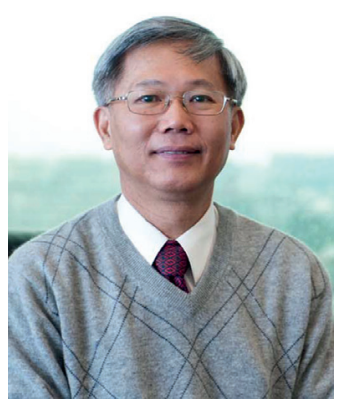

Chair

Pei-Jer Chen

Professor,

Graduate Institute of Clinical

Medicine, National Taiwan

University and Hospital, Taipei,

Taiwan which has established a hepatitis B viral load paradigm in the natural history of chronic hepatitis $B$. This discovery has led to a new clinical guideline for the management of chronic hepatitis $B$ to improve the treatment of million patients globally, especially in Asia-Pacific and Sub-Saharan countries. Prof. Chen also involves in the study of liver cancer prevention by hepatitis $B$ vaccination. This finding has led to the new era of vaccination against cancers. Prof. Chen has been invited as a member of the WHO advisory committee for cancer prevention by vaccination, a member of USA National Institutes of Health advisory committee for management of chronic hepatitis $B$, and an IARC working group member to evaluate the cancer risk associated with various viral infections. 


\title{
Session 6
}

\section{Future Treatment for HCC}

\author{
10:00-11:55, July 12, 2014 (Auditorium)
}

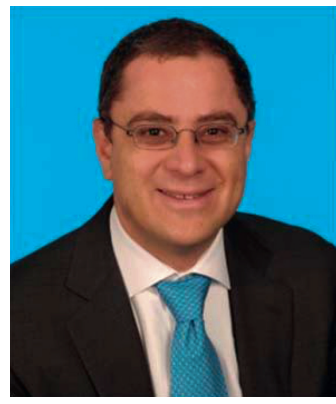

Chair

Ghassan K. Abou-Alfa

Professor,

Memorial Sloan-Kettering Cancer

Center, New York, USA

\section{Speech Title 1 \\ New Molecular Targeted Agents on the Horizon}

Joong-Won Park

National Cancer Center, Seoul, Korea

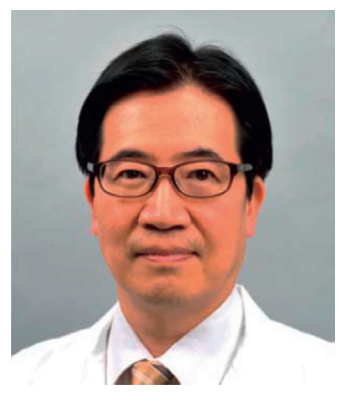

Prof. Park is a Principal Scientist of the National Cancer Center, Korea and a Professor of Graduate School of Cancer Science and Policy. He was the Head of the Center for Liver Cancer, NCC, Korea from 2002 to 2010, and was the Head of Translational and Clinical Research at the National Cancer Center Research Institute from 2008 to 2011. Prof. Park has published extensively in both International and Korean journals and given many invited lectures on hepatitis and liver cancer. He serves as Chair of the Committee for the Hepatocellular Carcinoma Management Guidelines of the Korea Liver Cancer Study Group (KLCSG)-NCC Korea and is a member of the Korean Association for the Study of the Liver (KASL), the Asian-Pacific Association for the Study of the Liver (APASL), the American Association for the Study of Liver Diseases (AASLD), and the International Liver Cancer Association (ILCA). He served as Chair of the Scientific Committee for the APASL 2008 Seoul meeting.

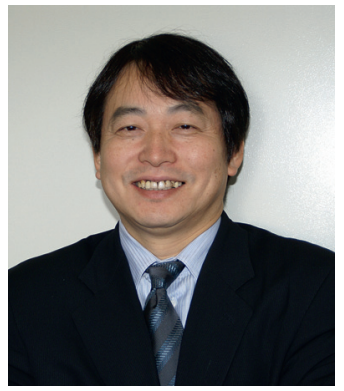

Chair

Masatoshi Kudo

Professor and Chairman,

Department of Gastroenterology

and Hepatology, Kinki University

School of Medicine, Osaka, Japan

\section{Abstract}

Hepatocellular carcinoma (HCC), the sixth most commonly occurring cancer, is ranked as the third leading cause of cancer death worldwide. Because a considerable number of HCC patients are diagnosed with advanced disease, development of systemic therapeutic agents is a serious unmet need under current conditions.

Although sorafenib is only one approved molecular targeted agent (MTA) for the advanced HCC, the agent is not widely used largely in Asia due to affordability, more frequent adverse events, and modest effects; a median time-toprogression (TTP) of 2.8 months and a median overall survival (OS) of 6.5 months in Asian trial. Interestingly, multiple phase III randomized controlled trials (RCT) recently showed an about 10-month median OS in the sorafenib treatment group. Unfortunately, recent multiple RCTs with potent antiangiogenic MTAs (sunitinib, brivanib, linifanib) resulted in failure and those suggest that this might be a pitfall of single-antiangiogenic MTA therapy. Overcoming this pitfall requires MTA of other mechanism or the combination of antiangiogenic MTA with cytototoxic chemotherapy, or locoregional therapy such as transarterial chemoembolization (TACE).

HCCs are highly vascular tumors and vascular endothelial growth factor (VEGF) is linked to hepatocarcinogenesis. Encouraged by the efficacy seen with sorafenib, several more selective and more potent antiangiogenic tyrosine-kinase inhibitors (TKIs) and antibodies are being developed in HCC; pazopanib in phase I, axitinib in phase II, lenvatinib and ramucirumab in phase III. Based on positive data from a phase II trial of sorafenib plus doxorubicin, a phase III RCT of sorafenib plus doxorubicin versus sorafenib alone is underway. Ongoing phase II trials include the combination of sorafenib with gemcitabine/oxaliplatin (GEMOX), modified FOLFOX, or capecitabine/oxaliplatin.

Signaling through the fibroblast growth factor (FGF)/ FGF receptor (FGFR) pathway may play a role in fibrosis of 
cirrhosis and hepatocarcinogenesis. The dual opportunity to possibly inhibit the progression of cirrhosis and hepatocarcinogenesis makes the FGF/FGFR pathway an attractive therapeutic target in HCC. Brivanib, a dual inhibitor of VEGFR and FGFR, is representative of the FGFR inhibitor. Despite positive phase II data in HCC, brivanib failed in the phase III RCT in both the first- and second-line settings in advanced HCC patients. Ongoing first-line trials of FGFR inhibitor include lenvatinib (phase III RCT), dovitnib (phase II RCT), and nintedanib in combination with sorafenib (phase I/II).

Emerging data provide evidence that the pathogenesis and progression of HCC are mediated by a number of molecular defects and deregulated pathways. Among those, deregulation of c-Met and HGF are common in HCC. c-MET inhibitors have shown modest efficacy in phase II trials. Tivantinib, a selective, non-ATP-competitive inhibitor of c-MET, showed improved TTP, particularly for patients with MET-high tumors. Cabozantinib (XL184), a RTK inhibitor of c-MET/ VEGFR2, demonstrated a median PFS of 4.2 months and is also undergoing phase III evaluation in patients where sorafenib failed or could not be tolerated.

The Wnt pathway have been shown to be involved in hepatocarcinogenesis and the regulation of cancer stem cells (CSCs). OMP-54F28 is a recombinant protein inhibiting Wnt signaling. A phase I/II trial of OMP-54F28 in combination with sorafenib for the first-line treatment of advanced HCC is ongoing.

mTOR regulates protein translation, angiogenesis, and cell cycle progression in many cancers including HCC. The mTOR inhibitor everolimus in a phase III RCT in the secondline treatment of advanced HCC patients unfortunately did not meet its primary endpoint of OS.

Current immunotherapy trials in advanced HCC patients include a phase I trial of tremelimumab, a human IgG2 monoclonal antibody to CTLA-4 in combination with chemoembolization or radiofrequency ablation. Additionally, histone deacetylase inhibitors, MEK inhibitors, inhibitors of the IGF/ IFGR pathway, and oncolytic viruses are under being studied in patients with advanced HCC.

\section{Speech Title 2 Immunotherapy for Hepatocellular Carcinoma (HCC): Anti-Tumor and Anti-Viral Effects}

Bruno Sangro

Clinica Universidad de Navarra, Pamplona, Spain

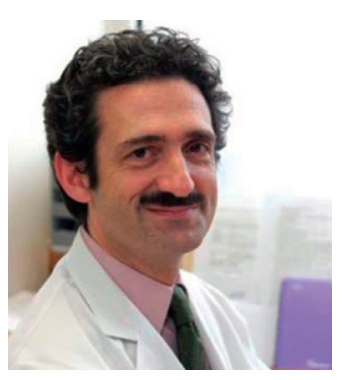

Prof. Sangro is Director of the Liver Unit at Clinica Universidad de Navarra, Aggregate Professor of Medicine at the University of Navarra School of Medicine, and senior researcher in the National Biomedical Research Network Center for Liver and Digestive Diseases (CIBERehd). He also serves in the Governing Board of the Division of Hepatology and Gene Therapy at the Center for Applied Biomedical Research (CIMA), in Pamplona, Spain, and is advisor for the Spanish and the European Agency for Medicinal Products. His research has mainly focused on liver cancer, with a high priority on therapeutic innovation. The Liver Unit of Clinica Universidad de Navarra has pioneered several approaches to the treatment of liver cancer from expanded criteria for liver transplantation to locoregional vascular therapy including intraarterial infusion chemotherapy, transarterial embolization and radioembolization, and systemic advanced therapies including gene therapy or immunotherapy.

\section{Abstract}

Newer immunotherapy agents may break the barrier that tumors create to evade the attack from the immune system. Dendritic cell vaccination has shown encouraging clinical activity and a favorable safety profile in advanced tumor stages. However, optimal cell maturation status, choice of tumor antigens, and route of administration have not been established. Single or multiple peptides derived from tumorassociated antigens may also be used for cancer vaccination. Intratumoral delivery of oncolytic viruses expressing immunostimulating cytokines like GM-CSF has produced stimulating clinical results that need further verification.

But it is probably T-cell checkpoint modulation that has recently attracted the highest expectations. In fact, targeting cytotoxic T-lymphocyte-associated antigen 4 (CTLA-4), programmed death 1 (PD-1) and programmed death ligand 1 (PD-L1) by use of monoclonal antibodies has revolutionized the field of cancer immunotherapy by showing substantial 
effect across a variety of tumor types. Tremelimumab is a human monoclonal antibodies that upon binding CTLA-4 enhances T-cell activation and proliferation through various mechanisms still under study, which may include partial intratumoral depletion of regulatory T cells. In a pilot phase II clinical trial targeting the population of HCC patients with chronic hepatitis $\mathrm{C}$ virus (HCV) infection, tremelimumab has recently shown signs of antitumor and antiviral activity. Twenty-one patients with mostly advanced tumors and varying degrees of liver dysfunction received tremelimumab at a dose of $15 \mathrm{mg} / \mathrm{kg}$ every 90 days until tumor progression or unacceptable toxicity. Treatment was overall well tolerated with an itching skin rash being the most frequent adverse event. Despite the reduced number of patients, revealing signs of efficacy were observed. Three patients (18\%) had a partial response and 10 patients (59\%) had a stable disease that in almost half of the cases lasted longer than 6 months. Furthermore, a $>50 \%$ drop in alpha-fetoprotein was observed in $36 \%$ of patients with high baseline levels (> $100 \mathrm{ng} / \mathrm{mL}$ ). Time to progression (median 6.5 months; $95 \%$ CI 3.95 to 9.14 ) compares well with most phase 2 trials of targeted agents and provides further evidence of antitumor activity. Besides this antitumor effect, tremelilmumab produced a decline in viral load and elicited specific $\mathrm{T}$ cell responses against hepatitis $\mathrm{C}$ virus antigens in most patients.

PD-1 receptor is expressed by T lymphocytes preferentially with long-term exposure to antigens and contributes to $\mathrm{T}$ cell exhaustion. Nivolumab is a human antibody that blocks PD-1 and has produced impressive durable objective responses in patients with melanoma, renal-cell cancer, and non-small-cell lung cancer. Following these results, a doseescalating phase II clinical trial is exploring the safety and efficacy of Nivolumab for the treatment of HCC, including cohorts that explore viral etiology cases. In a placebocontrolled single-ascending-dose study in patients with chronic HCV infection, 5 patients who received Nivolumab met the primary study endpoint of a reduction in HCV RNA $\geq 0.5 \log 10 \mathrm{IU} / \mathrm{mL}$ on at least 2 consecutive visits while 2 patients achieved HCV RNA below the lower limit of quantitation. Altogether, these data suggest that $\mathrm{T}$ cell checkpoint inhibitors may have substantial activity against HCC and HCV.

\section{Speech Title 3 Targeting Liver Cancer Stem Cells: Hype or Hope? \\ Stephanie $\mathrm{Ma}$ \\ Department of Anatomy, Li Ka Shing Faculty of Medicine, The University of Hong Kong, Hong Kong}

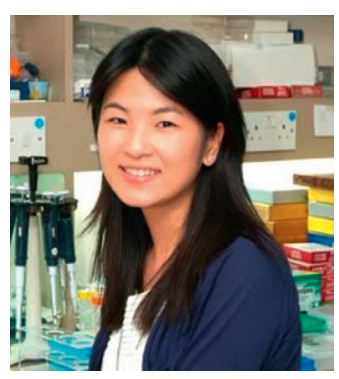

Prof. Ma is currently an Assistant Professor in the Department of Anatomy at the Faculty of Medicine of The University of Hong Kong. Her research interest focuses on the identification, characterization, OMIC profiling and therapeutic targeting of liver cancer stem cells. She is the recipient of numerous awards including the 2008 Hong Kong Young Scientist Award in Life Sciences (Hong Kong Institution of Science), the Faculty of Medicine Outstanding Research Output Awards (The University of Hong Kong) for two consecutive years in 2011 and 2012, as well as the 2013 Outstanding Young Researcher Award (The University of Hong Kong). Dr. Ma is listed as the top 1\% most cited scholars in 'Clinical Medicine' and 'All Fields' ranked by the prestigious ISI's Essential Science Indicators since 2010. She has published extensively in liver cancer stem cell research with original research findings in leading international journals including those of Cell Stem Cell, Journal of Clinical Investigation, Gastroenterology, Hepatology, Cancer Research and Oncogene. Dr. Ma is also a Principal Investigator of the State Key Laboratory for Liver Research and an active member of the Centre for Cancer Research at The University of Hong Kong.

\section{Abstract}

Cancer stem cells is a progressive concept to account for the phenotypic and functional heterogeneity in cancer cells. Despite the "controversies" regarding the cancer stem cell model, it has the potential to provide a foundation for new innovative treatment targeting the roots of cancer. There has been tremendous growth in liver cancer stem cell research studies in the last several years; and solid data do now exist to show that hepatocellular carcinoma (HCC) is at least in part dictated and maintained by a subset of cancer stem cells. There is also mounting evidence to show that this particular subset of cells are to blame for HCC relapse and drug resistance and thus targeting them can be an important aspect of HCC therapy in the future. In this talk, a summary of the recent work in the area with regard to the possibility and challenges of targeting liver cancer stem cells from different perspectives (biomarkers, signaling pathways, cancer stem cell niche) will be discussed. 


\title{
Session 7
}

\section{Can We Find New Therapeutic Targets for HCC?}

\author{
13:40-14:55, July 12, 2014 (Auditorium)
}

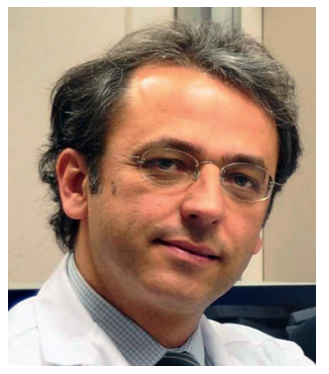

Chair

Josep M. Llovet

Professor,

Liver Unit, IDIBAPS-Hospital

Clinic of Barcelona, Barcelona,

Spain

Mount Sinai School of Medicine

New York University, New York, USA

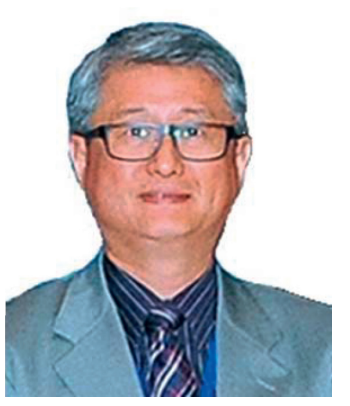

Chair

Yee Chao

Professor,

Cancer Centre Veterans General

Hospital, Taipei, Taiwan

\section{Speech Title 1 \\ Whole-Genome Sequencing Analysis in HBV- Related HCC in Asia}

Mao Mao

WuXi AppTec, China

Dr. Mao is as Senior Vice President of Translational Bioscience and Diagnostics at WuXi AppTec, the largest CRO in Asia. He has adjunct professorships in several academic institutions in Asia. Prior to joining WuXi, he held various positions at Pfizer and Merck. During his early years with Rosetta/Merck, he made significant contributions to the Ink Jet DNA microarray technology development and subsequent applications. He was one of the inventors of the 70-gene breast cancer prognosis test (MammaPrint), which became the world's first In Vitro Diagnostic Multivariate Index Assay (IVDMIA) to acquire market clearance from the US FDA in 2007. As one of the founding members of the National Human Genome Center in Shanghai, he established the first high-throughput DNA sequencing facility and pioneered genome research in China. He was also a principal investigator of National Key Programs of the 9th Five Year Plan in China. He has published over 60 articles in the peerreviewed journals including Nature.

\section{Abstract}

Hepatocellular carcinoma (HCC) is one of the most common cancers worldwide and yet the molecular basis of hepatocarcinogenesis remains largely unknown. NGS studies on HCCs have not only confirmed previously known mutations in CTNNB1 and TP53 in HCC, but also identified novel genetic alterations including JAK1 actionable mutations. These findings have started to depict a genetic landscape in HCC and facilitate development of novel therapeutics for the treatment of this deadly disease. In this talk, a published work of the Asian Cancer Research Group (ACRG) consortium will be introduced, together with some newly generated JAK1 mutation and in vivo pharmacology data.

\section{Speech Title 2 \\ Massive Cell Line Analyses to Find New Targeted Agents for HCC}

Alan Huang

Oncology Translational Medicine, Novartis Institute for Biomedical Research, Cambridge, USA

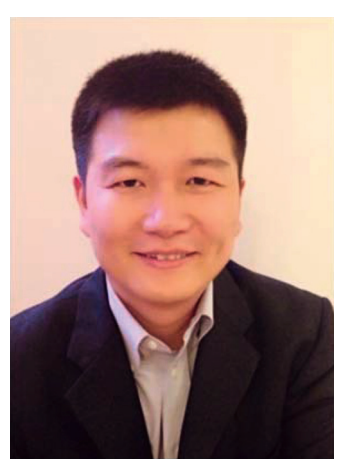

Dr. Huang is a Senior Director at Novartis Oncology Translational Medicine (OTM). In this role, he has been the scientific champion of numerous oncology clinical programs, including various PI3K inhibitors, mTOR inhibitors, HSP9O and cMET inhibitors by providing enabling pre-clinical rationales derived from cutting-edge experimental systems such as "Cancer Cell Line Encyclopedia". Dr. Huang is also overseeing OTM's molecular epidemiology and translational research effort of 
The 5th Asia-Pacific Primary Liver Cancer Expert Meeting (APPLE 2014)

Asian-Pacific diseases to support the clinical expansion in the region. Prior to formation of OTM in 2009, he has been working on target and drug discovery as a Research Investigator/Senior Research Investigator at NIBR Oncology Research for more than 6 years.

\section{Abstract}

Cancer Cell Line Encyclopedia (CCLE) is a compilation of gene expression, chromosomal copy number, next generation sequencing, proteomics and epigenetics data from 947 human cancer cell lines (Barretina J et al 2012). This systematic cancer genomic and pathway characterization, coupled with massive pharmacological profiling efforts, has enabled us to generate genetic and pathway prediction hypothesis of drug response in the preclinical setting that could be translated into clinical investigation in a speedy way. Hepatocellular carcinoma (HCC) is one of the leading cause of cancer related death worldwide, nonetheless, the understanding of the underlying molecular pathogenesis mechanism as well as successful therapeutic interventions are lacking in this highly un-met medical need. CCLE has provided us the opportunity to explore and identify of genetic and gene-expression-based predictors of drug sensitivity and combination strategies. Some of the key findings, including the predictive feature for mTOR inhibitor will be discussed. Features derived from the CCLE data were also further validated using patient tumor samples and patient derived primary xenograft models.

\section{Speech Title 3 \\ Molecular Targeted Therapies in HCC: Recent RCT Failures and Emerging Drugs}

Josep M. Llovet

Liver Unit, IDIBAPS-Hospital Clinic of Barcelona, Barcelona, Spain; Mount Sinai School of Medicine New York University, New York, USA

Prof. Llovet is Chairman of the European Clinical Practice Guidelines of management of liver cancer (EASL-EORTC) and serves as Senior Editor of Clinical Cancer Research and Special Editor of Gastroenterology and Journal of Hepatology. He contributes tremendously to advancing knowledge in both clinical and preclinical research of HCC, including (1) clinical classification of HCC (the BCLC classification); (2) establishment of chemoembolization as standard of care in patients with intermediate HCC; (3) establishment of sorafenib as standard of care in patients with advanced HCC; (4) molecular diagnosis and classification of HCC. Prof. Llovet has published more than 180 articles in peer-reviewed journals including New England Journal of Medicine, Nature Genetics, Lancet, Cancer Cell, Journal Clinical Investigation, etc. He has been awarded with the AACR-Landon International Award (2009), the Interna- tional Hans Popper award (2012), and Premi Josep Trueta (2013).

\section{Abstract}

Hepatocellular carcinoma (HCC) is a major health problem. Most HCC patients recur after resection/ablation or are diagnosed at advanced stages. Sorafenib remains the only approved systemic drug. Molecular therapies targeting signaling cascades involved in HCC development have been explored in phase III clinical trials. However, none of the drugs tested have shown positive results in first (brivanib, sunitinib, erlotinib and linifanib) or second line (brivanib, everolimus) after sorafenib progression. Reasons for failure are heterogeneous and include lack of understanding of critical drivers of tumor progression/dissemination, liver toxicity, flaws in trial design or marginal antitumoral potency. These trials are also challenging time to progression as a surrogate end-point of survival. Trials ongoing testing drugs head-to-head vs. sorafenib in "all comers" might have difficulties in achieving superior results in first line. Novel trials are also designed testing drugs in biomarker-based subpopulations of HCC patients. Two types of studies are proposed: 1) Phase II pivotal proof-of-concept studies testing drugs blocking potential oncogenic addiction loops, such as the one testing MEK inhibitors in RAS+ patients or amplification of FGF19 as a target, 2) Phase II-III studies using biomarker-based trial enrichment for defining HCC subpopulations, such as the case of enriching for MET-positive tumors. These strategies have deemed successful in breast, melanoma and lung cancer, and are expected to change the landscape of HCC trial design. 


\title{
Session 8
}

\section{Treatment of Advanced HCC: Exploring the Uncharted Territories}

15:15-16:25, July 12, 2014 (Auditorium)

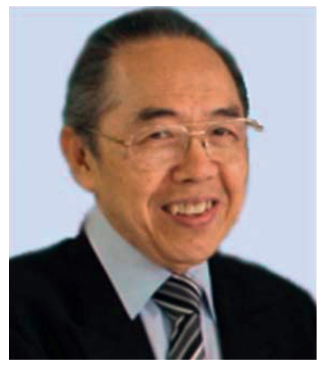

Chair

Laurentius A. Lesmana

Professor,

Division of Hepatology, University

of Indonesia, Jakarta, Indonesia

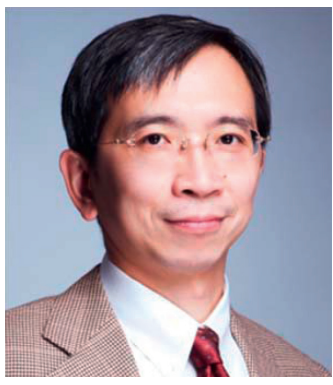

\author{
Chair \\ Chih-Hung Hsu \\ Associate Professor, \\ Graduate Institute of Oncology, \\ National Taiwan University \\ College of Medicine, Taipei, \\ Taiwan \\ Department of Oncology, National \\ Taiwan University Hospital \\ Taipei, Taiwan
}

\section{Speech Title 1 \\ Design Issues of Multi-Modality Trials in HCC \\ Peter R. Galle \\ University Medical Center Mainz, Mainz, Germany}

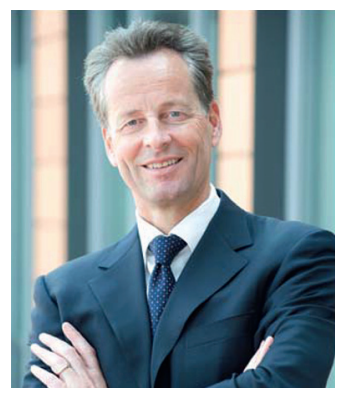

Prof. Galle received his M.D. degree from Marburg University and Ph.D. degree from Heidelberg University. He worked as postdoctoral fellow in Molecular Biology at the Centre for Molecular Biology Heidelberg, working on the replication of hepatitis B viruses. In 1998 he became Director of the I. Medical Department in Mainz and from 2005-2008 he hold the CEO position of Mainz University Hospital. Prof. Galle is the current President of the International Liver Cancer Association (ILCA). He serves as Co-editor for the Journal of Hepatology and is on the Editorial Boards of several other Journals. His research has focused on elucidating important aspects of apoptotic cell death in the liver, immune escape of tumour cells, and preclinical and clinical research in HCC. He was awarded several prizes, amongst others the prestigious Tannhauser award, the highest prize of the German Society for Digestive Diseases.

\section{Abstract \\ Background}

Treatment of advanced HCC remains a challenge. Sorafenib has been established as standard of care after it had been demonstrated to significantly improve overall survival. However, the gain in live expectancy has been modest. Meanwhile six phase III trials in advanced stage have failed to reach their primary endpoint, further demonstrating the difficulties to treat HCC.

One option to improve therapeutic outcome is the combination of loco-regional and systemic therapy. Today limited data are available on the combination of TACE with Sorafenib. Most data have been developed in the setting of intermediate stage HCC, where Sorafenib is added to TACE. Little data are available on advanced stage HCC, adding TACE to Sorafenib.

Rationale for Combined Treatment Approaches

BCLC-B: Most patients progress after TACE as a noncurative treatment approach resulting in a 3-year survival rate $<30 \%$. The extent of necrosis after TACE is dependent on the embolized vessels; the peripheral part of the tumor has a tendency to survive TACE. Hypoxia in the surviving margin may upregulate growth- and angiogenic-factors (e.g. HIF-1 $\alpha$, VEGF, IGF-2). High levels of angiogenic factors are associated with poor outcome and metastatic progression. This could be counterbalanced by additional treatment with Sorafenib.

BCLC-C: The addition of TACE to Sorafenib in advanced stage HCC provides an option of local control, taking into consideration that intrahepatic tumor progression is survival limiting.

\section{Where Do We Stand?}

Current phase I-III trials with combined treatment approaches are heterogeneous and comprise different endpoints (TTP, OS, safety), different times of randomization, different TACE procedures (conventional [CTACE] vs. doxorubicin-eluting beads [DEB-TACE]) and schedules (predefined vs. on-demand), heterogeneous sorafenib schedules (sequential 
The 5th Asia-Pacific Primary Liver Cancer Expert Meeting (APPLE 2014)

vs. interrupted vs. continuous), heterogeneous patient selection (BCLC B vs. BCLC C), and heterogeneous liver function (ChildPugh A vs. Child-Pugh B). Based on this heterogeneity the results are inconclusive.

\section{Future Perspectives and Challenges}

Trials will have to be harmonised in order to reduce the confounding impact of patient and treatment factors.

Patient selection: It will be necessary to exclude large, diffuse tumors poorly responding to TACE. Assessment of combined treatment of advanced HCC (BCLC C) will further require the exclusion of Child-Pugh $\mathrm{B}$ patients and of patients with segmental portal vein invasion.

Treatment: It may be better to avoid predefined schedules versus TACE-on-demand (real-life practice!) and it needs to be decided whether to apply cTACE vs DEB-TACE (real-life practice!) and continuous (overlapping side effects) vs interrupted schedules (maximizing the potential efficacy).

\section{Speech Title 2 \\ Is There A Role of Cytotoxic Chemotherapy in Advanced Unresectable Hepatocellular Carcinoma?}

Winnie Yeo

Department of Clinical Oncology, Chinese University of Hong Kong, Hong Kong

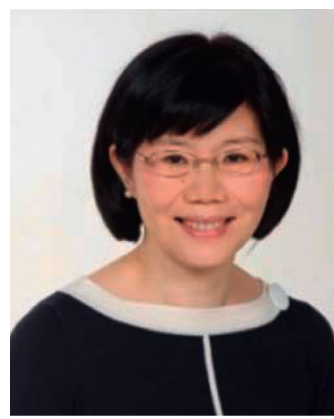

Prof. Yeo is currently a clinical professor of the Department of Clinical Oncology, Faculty of Medicine, the Chinese University of Hong Kong. Prof. Yeo has been the Chairman of the Medical Oncology Specialty of the Hong Kong College of Physicians between 2007 and 2013. She serves as a member and advisor in various expert panels and committees within the University, the Hong Kong Hospital Authority and various health advisory panels. She has authored and coauthored over 180 papers and her main research interests are management of liver, breast and gastric cancer patients as well as hepatitis B virus-related complications in cancer patients.

\section{Abstract}

Hepatocellular carcinoma (HCC) is the second most common cause of cancer-related death in men and sixth in women worldwide. At present, only surgical resection and liver transplantation are considered curative measures, in terms of disease-free survival. However, these approaches can only be applied to patients with early stage HCC. A significant proportion of patients diagnosed with HCC are not amenable to a curative resection, or eventually progress despite locoregional treatments at which point systemic treatment, with a palliative intent, is considered. To date, the only systemic agent that has been shown to provide survival benefit over best supportive care is the multikinase inhibitor of VEGFR, PDGFR and Raf, sorafenib. However, the overall prognosis of HCC patients remains poor. There is thus a need to identify better therapies for patients with advanced disease.

Prior to the era of targeted therapies, the efficacy of systemic cytotoxic chemotherapy had been widely assessed in patients with advanced HCC. Unfortunately, trials on systemic chemotherapy had been offset by two limitations. First, most trials were conducted in small scale, in the format of single arm phase II setting. Doxorubicin, whether in combination to other agents or as a single treatment, is the most commonly studied cytotoxic agent in HCC. Doxorubicin has shown response rates of $10-20 \%$ in clinical trials. However, apart from one early randomized study which showed some survival advantage in patients who were treated with doxorubicin when compared to those did not, there had been no other studies to confirm such finding. Unfortunately, the potential benefit was outweighed by the treatment-related toxicities. Cytotoxic chemotherapy is associated with significant side effects, and for HCC patients in our geographical areas, the disease is often associated with chronic hepatitis $\mathrm{B}$ or $\mathrm{C}$ virus infections; as such the tolerability to chemotherapy may be compromised by the concomitant chronic liver diseases. Supportive therapies, especially with regards to anti-viral therapies for hepatitis B infected HCC patients were suboptimal until the last decade.

In this talk, data on phase II trials that assessed various cytotoxic agents either as single agent or in combination regimen, as well as phase III trials that assessed newer cytotoxic agent or combination regimen in comparison with single agent doxorubicin, and the possibilities of combining cytotoxics with novel targeted agents will be discussed. 
Speech Title 3

\section{Radiotherapy; Will It Have A Place in the} Guidelines of Hepatocellular Carcinoma?

Jinsil Seong

Department of Radiation Oncology, Yonsei University

College of Medicine, Seoul, Korea

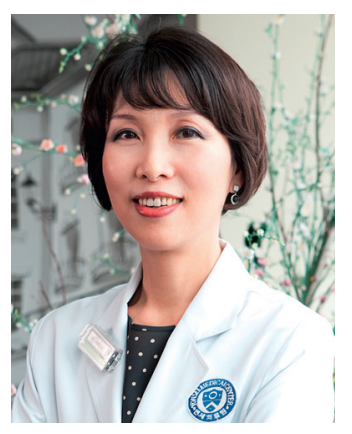

Prof. Seong is a former President of Yonsei Liver Cancer Study Group She served as a member of the Editorial Board of International Journal of Radiation Oncology Biology Physics for the past 10 years. Currently she is a council member of Asian Clinical Oncology Society as well as Asia Pacific Primary Liver Cancer Expert Meeting. She is also working as a consultant in International Atomic Energy Agency. She has bee awarded many times for her credit including Korean Cancer Association Distinguished Scientific Award (2012), Best Presentation Award (bronze) in $2^{\text {nd }}$ Asia Pacific Primary Liver Cancer Expert Meeting (2011) and Young Investigator Award in International Congress of Liver Disease (2004). Prof. Seong has published 97 articles in non-SCI listed journals and 103 articles in SCI listed journals. She has also delivered 106 talks at major national and international congresses.

\section{Abstract}

Therapeutic decision in hepatocellular carcinoma (HCC) is determined by tumor staging, preferentially adopting BCLC staging and guideline system. BCLC system clearly defines treatment modality that can be recommended in each stage; potentially curative therapies for HCC are well established for early stages and sorafenib, for advanced stage.

However, there are a plenty of occasion, in which clinical outcome can be further improved within each subset of BCLC stages. Radiotherapy can either be complementary to weakness of BCLC-recommended treatment or be effective as alone. Current radiotherapy technology has evolved remarkably during the past decade. It can be precisely delivered, thereby permitting higher doses to the tumor and reduced doses to surrounding normal tissues.

According to the Korean Liver Cancer Study Group (KLCSG) practice guidelines, radiation therapy is considered as one of options for unresectable, locally advanced HCC with level II evidence. In some situations where other popular local approaches wouldn't be feasible, radiotherapy is most recommended option. Based on accumulated experiences and evidences, importance of radiotherapy is recognized more widely and increasing number of institutes apply radiotherapy in clinic. Radiotherapeutic strategies will be further discussed according to each clinical setting for patients with HCC. 


\section{Session 9}

\section{Asian Perspectives for Pivotal Trials of HCC: Why Data Are Different?}

08:00-09:40, July 13, 2014 (Auditorium)

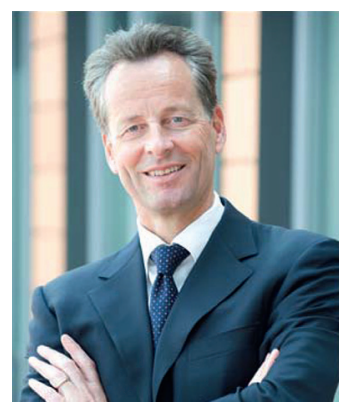

Chair

Peter R. Galle

Professor,

University Medical Center Mainz,

Mainz, Germany

\section{Speech Title 1 \\ Adjuvant Therapy after Surgery/Ablation}

Ronnie T.P. Poon

The University of Hong Kong, Division of Hepatobiliary and Pancreatic Surgery, Queen Mary Hospital, Hong Kong

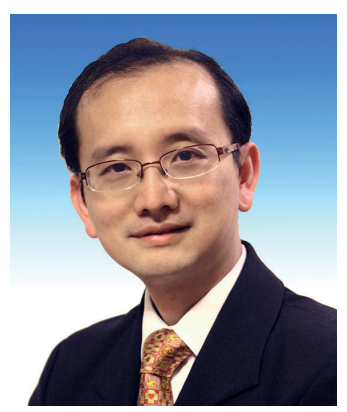

Prof. Poon is the bearer of Suen Chi-Sun Endowed Professorship in the University of Hong Kong. He is currently the Chief of the Division of Hepatobiliary and Pancreatic Surgery at Queen Mary Hospital. Prof. Poon has devoted much of his research effort on management of liver cancer, ranging from surgical resection or ablation for early cancer to molecular targeted therapy for advanced disease. He has recently developed the Hong Kong Liver Cancer Staging System and contributed to the establishment of consensus guidelines on management of liver cancer by various international organizations. Prof. Poon has served as the Chairman of the Scientific Committee of the International Hepato-Pancreato-Biliary Association. He is the Founding Board and Governing Council Member of the International Liver Cancer Association, and Secretary of the Asia-Pacific Primary Liver Cancer Expert Association. He is also the founder and Chairman of the Hong Kong

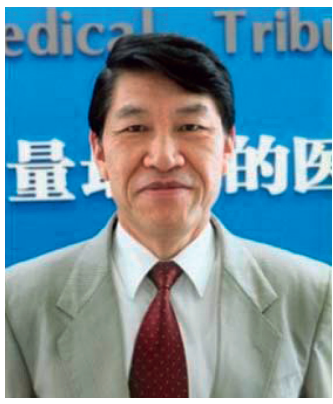

Chair

Sheng-Long Ye

Professor,

Liver Cancer Institute, Zhongshan

Hospital, Fudan University,

Shanghai, China
Liver Cancer Foundation. He has served as Associate Editor of the World Journal of Surgery, and he also serves in the editorial board of several journals including Cancer Therapy, World Journal of Gastroenterology, HBP Surgery, Journal of Gastrointestinal Surgery and Annals of Surgical Oncology.

\section{Speech Title 2 \\ Lessons from the TACE Trials \\ Masatoshi Kudo}

Department of Gastroenterology and Hepatology, Kinki University School of Medicine, Osaka, Japan

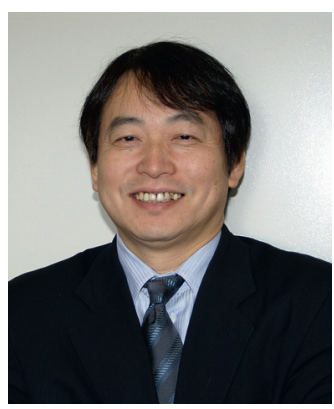

Prof. Kudo is currently a Professor and Chairman at the Department of Gastroenterology and Hepatology, Kinki University School of Medicine since 1999 and a President of Kinki University Medical Center since 2008. He has published 476 International scientific peer review papers in wellregarded journals in addition to 786 domestic scientific papers. Prof. Kudo serves as an Executive Council Board Member for Liver Cancer Study Group of Japan (LCSGJ), Chairman of Nationwide Survey Committee of LCSGJ, and a representative 
of LCSGJ Head Office. He is also an Executive Board Member of Japan Society of Hepatology (JSH), a Founding Board member of International Liver Cancer Association (ILCA), and the Editor-in-Chief of LIVER CANCER (Karger). Prof. Kudo's research interest is "Diagnosis and treatment of HCC". He is the first author of "Consensus-based Practice Manual of HCC Proposed by Japan Society of Hepatology" published in 2007 and 2010 revised version.

\section{Abstract}

Introduction:

There have been 3 clinical trials of sorafenib combined with or adjuvant therapy to transarterial chemoembolization (TACE). All of 3 trials showed negative results. In this lecture, what we learned from these 3 TACE combination trials will be presented.

\section{Clinical Trials:}

1. Post TACE phase III trial $(\mathrm{n}=458)$ conducted in Japan and Korea did not meet its primary endpoint of prolonging time to progression (TTP) after TACE in sorafenib treated group. However, subset analysis clearly showed that Korean patients with sorafenib group showed better TTP than those with placebo, whereas Japanese patients could not show the difference. Median treatment duration of sorafenib in Japan and Korea were 16.1 weeks and 30.9 weeks, respectively. Hazard ratios for TTP in Japan and Korea were 0.94 and 0.38, respectively (Kudo M. Eur J Cancer, 2011).

2. SPACE phase II trial $(\mathrm{n}=307)$ was conducted in USA and Asian countries except Japan. Drug eluting beads (DEB) was used as an embolic agent and scheduled TACE was adapted. Since this trial was phase II, $\alpha$ value was set as 0.15 . Therefore, primary endpoint of prolonging TTP of sorafenib combination over placebo was met, however HR was 0.797 $(\mathrm{p}=0.072)$

This does not seem positive result since median TTP in patients with sorafenib and that with placebo was 5.6 month and 5.5 month, respectively. Subset analysis clearly showed that median treatment duration of sorafenib in Asian patients (30.3 weeks) was longer than that in non-Asian patients (17.4 weeks), resulting in better hazard ratio for overall survival in Asian patients (HR: 0.678) than that (HR: 1.062) in non-Asian patients.

3. BRISK-TA trial $(n=502)$ is a randomized, doubleblind, multicenter phase 3 study of brivanib versus placebo as adjuvant therapy to TACE in patients with unresectable HCC. However, this study was terminated 2 years early when phase 3 studies of brivanib as first-and second line treatment in advanced HCC patients failed to meet their primary end point of OS. Therefore, this data are preliminary and immature. Although some positive signals such as time to extrahepatic spread or vascular invasion in patients with brivanib being better than that in patients with placebo, this study did not meet its primary endpoint of OS. Post-hoc analysis of treatment duration revealed shorter treatment duration (2.1 months in Japan) resulted in worse hazard ratio for OS (0.86 in Japan) as compared with longer treatment duration (10.1 months in Korea) and better hazard ratio (0.55 in Korea).

\section{Conclusion:}

What we learned from the 3 negative TACE trials is that longer treatment duration of sorafenib resulted in the better result.

\section{Speech Title 3 \\ Asian Subgroup Analysis of the Brivanib vs. Sorafenib Trial}

Jeong Heo

Internal Medicine, Pusan National University School of Medicine, Busan, Korea; Gastroenterology and Hepatology, Pusan National University Hospital, Busan, Korea

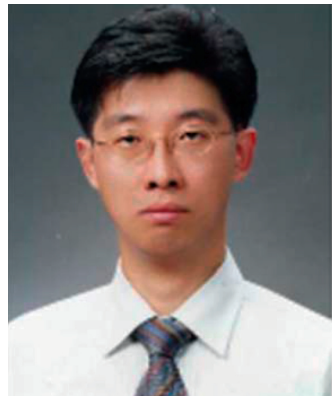

Prof. Heo is Director of Gastroenterology and Hepatology at Pusan National University Hospital. During his career, Prof. Heo held a number of academic positions, university and hospital appointments as well as principal investigator in many clinical trials for Phase I-IV of hepatitis B, C and hepatocellular carcinoma. He has also received research grants from the Korean Ministry of Education, Science and Technology. Prof. Heo has published more than 100 peer-reviewed manuscripts.

\section{Abstract}

Sorafenib (SOR) is only approved drug as a standard systemic therapy in advanced hepatocellular carcinoma (HCC), which is an oral multi-tyrosine kinase inhibitor that targets Raf/MEK/ERK, VEGF receptor and PDGF receptor that have given a next step for further development of HCC treatment. However, given marginal efficacy of the drug, especially in Asian-pacific area, many new molecular targeted agents have been tried with different pathways involved in hepatocarcinogensis. Brivanib (BRIV) was one potent and promising candidate of them, which is a dual inhibitor of vascular-endothelial growth factor and fibroblast growth factor receptors that are implicated in pathogenesis of HCC. A large multinational, randomized, double-blind, phase 3 trial compared BRIV with SOR as first-line treatment for HCC has 
given many experiences and informations according to areas and countries.

Patients were randomized (1:1) to BRIV (800 mg) once daily orally plus placebo or SOR (400 mg) twice daily orally plus placebo. Primary endpoint was overall survival (OS). Secondary efficacy and safety endpoints were evaluated. A total of 1,155 patients were randomized (Asia overall, $\mathrm{n}=718$ [62\%], with 223 [19\%] from China; non-Asia, $n=437$ [38\%]). Baseline characteristics were balanced between treatment arms. The study did not meet its primary objective of demonstrating OS non-inferiority for BRIV vs. SOR since the upper limit of $95 \%$ confidence interval (CI) for the hazard ratio (HR) exceeded the non-inferiority boundary (1.08) in the per protocol population (HR, 1.06; 95\% CI, 0.93-1.22). OS results for BRIV vs. SOR was consistent between the intent-to-treat (ITT) population (9.5 vs. 9.9 mos; HR, 1.07; 95\% CI, $0.94-$ 1.23) and Chinese (7.9 vs. 8.9 mos; HR, 1.18; 95\% CI, 0.871.62), Asian (8.7 vs. 8.9 mos; HR, 1.08; 95\% CI, 0.91-1.28), and non-Asian (10.9 vs. 11.8 mos; HR, 1.11; 95\% CI, 0.881.40 ) subgroups (table 1$)$. The most frequent $(\geq 5 \%$ in either arm) grade 3-5 adverse events (AEs) from any cause for BRIV and SOR were hyponatremia $(24 \%, 10 \%)$, fatigue $(15 \%, 7 \%)$, hypertension $(14 \%, 5 \%)$, decreased appetite $(8 \%, 3 \%)$, proteinuria $(5 \%, 1 \%)$, and hand-foot skin reaction $(2 \%$, $15 \%$ ). Discontinuation for AEs was $43 \%$ for BRIV and $33 \%$ for SOR.

Survival results with BRIV vs. SOR were consistent among the ITT, Chinese, Asian, and non-Asian groups. BRIV had an acceptable safety profile, but was generally less well-tolerated than SOR. This analysis will guide the development of new drugs for the treatment of HCC in terms of different areas and countries.
Table 1. Exploratory analysis: overall survival in geographic subsets

\begin{tabular}{|c|c|c|c|}
\hline & \multicolumn{2}{|c|}{$\begin{array}{l}\text { Median OS, months } \\
\text { (events/patients) }\end{array}$} & \multirow[t]{2}{*}{$\operatorname{HR}(95 \% \mathrm{CI})^{\mathrm{a}}$} \\
\hline & Sorafenib & Brivanib & \\
\hline ITT Population & $\begin{array}{l}9.9 \\
(412 / 578)\end{array}$ & $\begin{array}{l}9.5 \\
(425 / 577)\end{array}$ & $\begin{array}{l}1.07 \\
(0.94-1.23)\end{array}$ \\
\hline Non-Asia & $\begin{array}{l}11.8 \\
(131 / 206)\end{array}$ & $\begin{array}{l}10.9 \\
(157 / 231)\end{array}$ & $\begin{array}{l}1.11 \\
(0.88-1.40)\end{array}$ \\
\hline Asiab $^{\mathrm{b}}$ & $\begin{array}{l}8.9 \\
(281 / 372)\end{array}$ & $\begin{array}{l}8.7 \\
(268 / 346)\end{array}$ & $\begin{array}{l}1.08 \\
(0.91-1.28)\end{array}$ \\
\hline China & $\begin{array}{l}8.9 \\
(87 / 122)\end{array}$ & $\begin{array}{l}7.9 \\
(77 / 101)\end{array}$ & $\begin{array}{l}1.18 \\
(0.87-1.62)\end{array}$ \\
\hline Japan & $\begin{array}{l}11.6 \\
(34 / 52)\end{array}$ & $\begin{array}{l}12.8 \\
(38 / 55)\end{array}$ & $\begin{array}{l}1.00 \\
(0.62-1.60)\end{array}$ \\
\hline Korea & $\begin{array}{l}8.4 \\
(58 / 67)\end{array}$ & $\begin{array}{l}7.4 \\
(60 / 70)\end{array}$ & $\begin{array}{l}0.88 \\
(0.61-1.27)\end{array}$ \\
\hline Taiwan & $\begin{array}{l}9.4 \\
(57 / 72)\end{array}$ & $\begin{array}{l}9.2 \\
(50 / 65)\end{array}$ & $\begin{array}{l}0.94 \\
(0.64-1.38)\end{array}$ \\
\hline
\end{tabular}

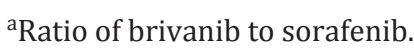

bPatient numbers were low in some Asian subsets.

\section{References}

Johnson PJ, Qin S, Park JW, et al: Brivanib versus sorafenib as first-line therapy in patients with unresectable, advanced hepatocellular carcinoma: results from the randomized phase III BRISK-FL study. J Clin Oncol 2013;31:3517-3524.

2. Cheng AL, Qin S, Park JW, et al: First-line brivanib versus sorafenib in unresectable, advanced hepatocellular carcinoma: Asia/non-Asia survival results in phase 3 BRISK-FL study. APASL 2013. 


\section{Session 10}

\section{APPLE Consensus Workshop: Searching Consensus for Controversies}

10:15-11:45, July 13, 2014 (Auditorium)

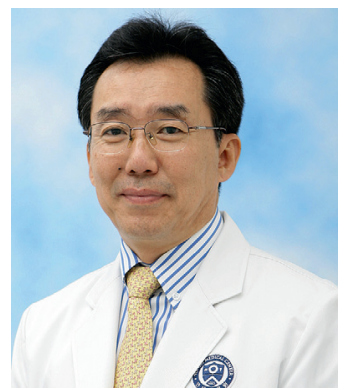

Chair

\section{Kwang-Hyub Han}

Professor and Chairman, Internal Medicine, Yonsei University College of Medicine, Seoul, Korea

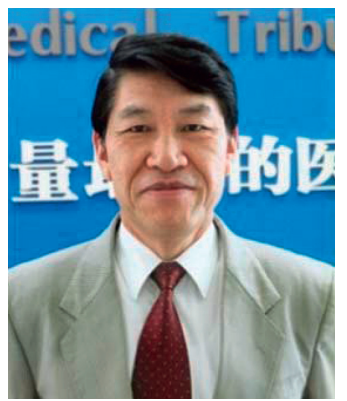

\section{Chair}

\section{Sheng-Long Ye}

Professor,

Liver Cancer Institute, Zhongshan

Hospital, Fudan University,

Shanghai, China

\section{Speech Title 1}

\section{Surgery for Intermediate/Advanced Stage} HCC

Norihiro Kokudo

Hepato-Biliary-Pancreatic Surgery Division and Artificial Organ and Transplantation Division, Department of Surgery, University of Tokyo Hospital, Tokyo, Japan

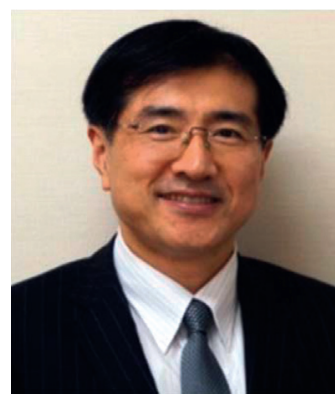

Prof. Kokudo's research interest focuses on surgical treatment of HCC, colorectal liver metastases, and living donor liver transplantation. Prof. Kokudo is currently the President of

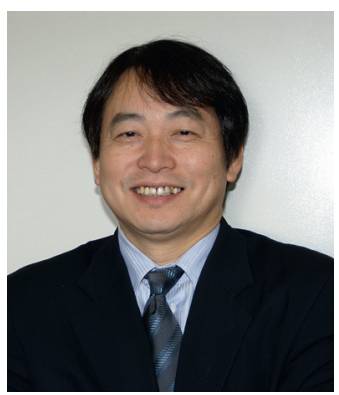

Chair

Masatoshi Kudo

Professor and Chairman,

Department of Gastroenterology and Hepatology, Kinki University School of Medicine, Osaka, Japan
Japan Surgical Society and the president elect for Asian-Pacific Hepato-Pancreato-Biliary Association (A-PHPBA). He oversaw the compilation of the $3 r d$ version of Japanese clinical practice guidelines for HCC in 2013. He is an associate editor of Hepatology Research and Liver Cancer, and on the editorial board of many scientific journals, including Annals of Surgery and World Journal of Surgery. 


\section{Speech Title 2}

\section{Prevention of HCC after Curative Therapy}

\section{Ji-Dong Jia}

Liver Research Center, Beijing Friendship Hospital, Capital Medical University, Beijing, China

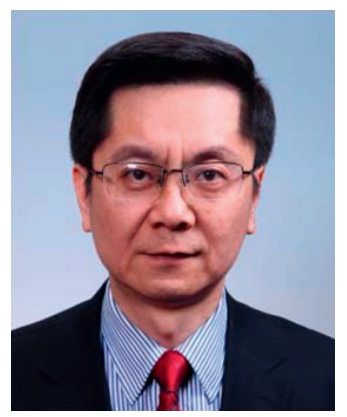

Prof. Jia's clinical interests include chronic viral hepatitis and autoimmune liver diseases. His research focuses on cellular and molecular biology of the extracellular matrix of the liver and the pathogenesis and therapeutic study of experimental liver fibrosis. Prof. Jia currently serves as vice president for the China Foundation of Viral Hepatitis Prevention and Control. He is also immediate past President of the Chinese Society of Hepatology, Past President of APASL (2009-2010), and Vice President of IASL. Prof. Jia serves as associate editor for Journal of Gastroenterology \& Hepatology, Hepatology International and Liver International.

\section{Speech Title 3 \\ Optimizing Imaging Diagnosis}

Takamichi Murakami

Department of Radiology, Kinki University Faculty of Medicine, Osaka, Japan

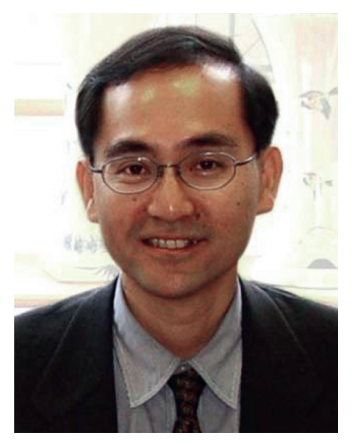

Prof. Murakami's research interest is "Radiological diagnosis and Interventional Radiology of abdominal disease", including dynamic MDCT and MR studies for detection and diagnosis of liver tumor, imaging evaluation of segmental liver function, and image navigation system to improve efficacy of local treatment for HCC. Prof. Murakami has published more than 500 articles and is on the review board of 14 peer-review journals, including American Journal of Roentgenology, Investigative Radiology, Journal of Magnetic Resonance Imaging, and European Radiology.

\section{Speech Title 4 \\ Radiotherapy-Current Practice and Future Clinical Trials \\ Jinsil Seong \\ Department of Radiation Oncology, Yonsei University College of Medicine, Seoul, Korea}

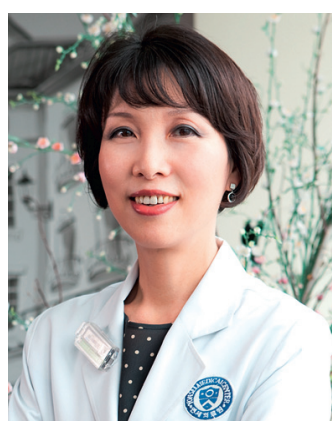

Professor Seong is a former President of Yonsei Liver Cancer Study Group and served as a member of the Editorial Board of International Journal of Radiation Oncology Biology Physics for the past 10 years. Currently she is a council member of Asian Clinical Oncology Society and the Asia Pacific Primary Liver Cancer Expert Meeting. She also works as a consultant in International Atomic Energy Agency. She has been awarded many times for her credit including Korean Cancer Association Distinguished Scientific Award (2012), Best Presentation Award (bronze) in 2nd Asia Pacific Primary Liver Cancer Expert Meeting (2011) and Young Investigator Award in International Congress of Liver Disease (2004). She has published 97 articles in non-SCI listed journals and 103 articles in SCI listed journals. Research interest is "Radiotherapy of HCC" in clinical approach as well as in translational research. 


\section{Speech Title 5}

\section{The Roles of Cytotoxic Chemotherapy}

Winnie Yeo

Department of Clinical Oncology, Faculty of Medicine, the Chinese University of Hong Kong, Hong Kong

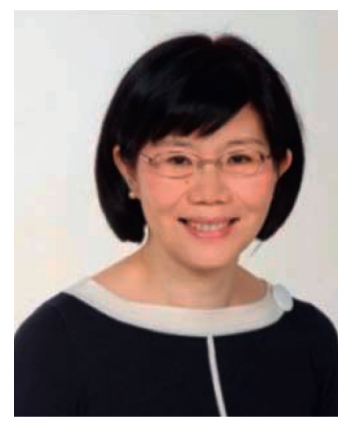

Prof. Yeo is currently a clinical professor of the Department of Clinical Oncology, Faculty of Medicine, the Chinese University of Hong Kong. Prof. Yeo has been the Chairman of the Medical Oncology Specialty of the Hong Kong College of Physicians between 2007 and 2013. She serves as a member and advisor in various expert panels and committees within the University, the Hong Kong Hospital Authority and various health advisory panels. She has authored and coauthored over 180 papers and her main research interests are management of liver, breast and gastric cancer patients as well as hepatitis B virus-related complications in cancer patients. 


\section{Satellite 1}

\section{Current Evidence and Efforts to Integrate Radiotherapy into HCC Guidelines}

10:30-12:00, July 11, 2014 (Song Bo Room)

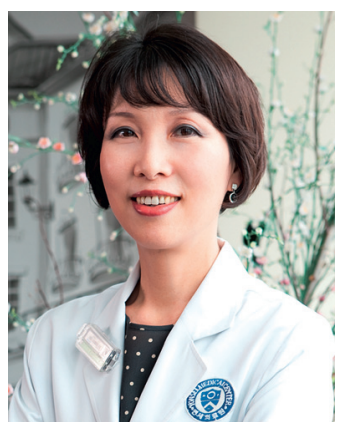

Chair

Jinsil Seong

Professor,

Department of Radiation

Oncology, Yonsei University

College of Medicine, Seoul, Korea

\section{Speech Title 1}

\section{IMRT and Other Novel Modalities}

Jason Chia-Hsien Cheng

Division of Radiation Oncology, National Taiwan

University Hospital Taipei, Taiwan

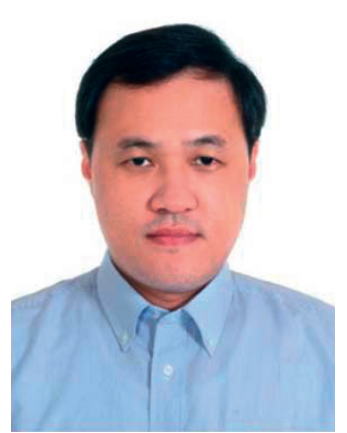

Prof. Cheng is currently a Full Professor at Graduate Institute of Oncology, National Taiwan University College of Medicine. He serves as a Principal Investigator of Affiliated Member Site of National Taiwan University Hospital for Radiation Therapy Oncology Group (RTOG) and is a Panel Member of Asian Consensus Statement for National Comprehensive Cancer Network (NCCN). Prof. Cheng also serves as the Associate Editor of International Journal of Radiation, Oncology, Biology, Physics and Journal of Radiation Oncology. His research interest is "Radiation Sensitizer and Radiotherapy Related Translation Research in HCC". He was the first author of "Radiation enhanced hepatocellular carcinoma cell invasion with MMP-9 expression through PI3K/Akt/NF- $\kappa B$ signal transduction pathway" published in Oncogene in 2006, which have been frequently cited for articles on metastasis. He is also the

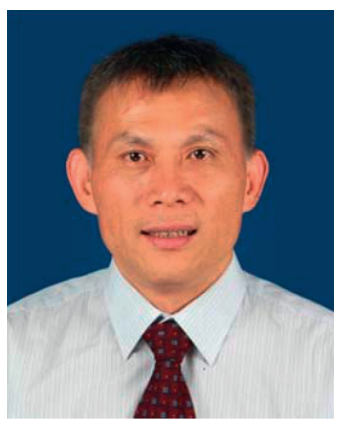

Chair

Zhao-Chong Zeng

Professor and Chairperson, Department of Radiation Oncology, Zhongshan Hospital, Fudan University, Shanghai, China

corresponding author of "MMP-9 from sublethally irradiated tumor promotes Lewis lung carcinoma cell invasiveness and pulmonary metastasis" which is published in Oncogene in 2012.

\section{Abstract}

As radiotherapy (RT) is being integrated into multimodality treatment for hepatocellular carcinoma (HCC), various modalities, including external RT and internal RT, have been implemented for this purpose. External RT has been widely used with either long-fractionated treatments or higher dose-per-fraction and fewer fraction-number stereotactic body radiation therapy (SBRT). Internal RT mostly uses yttrium-90 microsphere by embolization. It has been concerned of the altered patterns of failure. Despite the acceptable local control in the irradiated hepatic tumor, a certain proportion of patients develop intrahepatic and/or extrahepatic metastasis. The early published series using 1.8-2 Gy per fraction reported more than half of patients with metastasis out of RT field. Such an RT associated metastasis, especially in the liver, compromises the hepatic reserve and offsets the survival. Rare complete response and most partial response or stable disease of irradiated tumors indicate the sublethal effect of long fractionated RT. Higher-intensity RT by higher doseper-fraction and fewer fraction-number SBRT, and by higher-dose proton therapy demonstrates improved control of irradiated tumor and somehow reduced incidence of outof-field metastasis. The developing radiation sensitizers, such as histone deacetylase inhibitor, aurora kinase inhibitor, PI3K/mTOR inhibitors, and Sonic Hedgehog inhibitor may shed light in potentially improved effect on HCC when combined with RT from the preclinical studies. 
The 5th Asia-Pacific Primary Liver Cancer Expert Meeting (APPLE 2014)

The proposed RTOG trial to combine RT with sorafenib is also aimed to target on the RT activated signaling pathways for the radio-resistance and metastasis of HCC. Given no settled role of RT in the treatment guidelines for HCC, Asian cooperative group for multi-center trial is needed to form the consensus and establish evidence.

\section{Speech Title 2 \\ Clinical Results and Future Direction of Proton Beam Therapy for Hepatocellular Carcinoma}

Tetsuo Akimoto

Division of Radiation Oncology and Particle Therapy, National Cancer Center Hospital East, Chiba, Japan

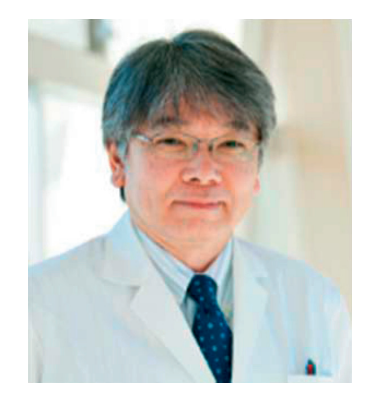

Dr. Akimoto is currently the Vice Director and Division Head, Division of Radiation Oncology and Particle Therapy, National Cancer Center Hospital East, Chiba, Japan. He is also a Professor at Tokyo Women's Medical University since 2010. Dr. Akimoto's research interest includes novel technology of radiation therapy and radiation biology for solid cancers. He has published 80 papers in his research field in international, peerreviewed scientific journals.

\section{Abstract}

The standard treatment option for hepatocellular carcinoma (HCC) are consisted from surgery, transcatheter arterial embolization (TAE), radiofrequency ablation (RFA) and hepatic transplantation according to the stages, liver function and tumor locations. Photon radiation therapy (RT), especially stereotactic body RT, is also a treatment option although indication is limited due to excessive dose to the normal liver. Proton beam therapy (PBT) enables us to deliver more conformal radiation to the target due to its unique physical character called as Bragg Peak. The results of the phase II study in National Cancer Center Hospital East (NCCHE) demonstrated that a 2-tear local control rate of $96 \%$ in treatment of 76GyE in 20 fractions, and a favorable liver function reserve that was determined by the ICG R15 was related to good overall survival. The similar treatment outcomes have been reported from the Tsukuba University.
Main advantage of PBT compared with surgery is non-invasive nature of PBT, indicating that PBT can be applied for patients with older ages or who are not suited for surgery due to commodities. In Japan, more than 3000 new patients were treated with PBT in 2012, and about $20 \%$ of all patients were HCC. Hence, particle therapy including PBT is considered to be an established treatment approach for HCC, although there has been no prospective trials evaluated the efficacy or local control compared with that after surgery. From these backgrounds, we are now planning the multi-institutional study that compared survival rate between PBT and surgery in the patients with resectable HCC. In this symposium, we will present advantages and effectiveness of PBT for patients with HCC, and summarize the current status of PBT in the treatment of HCC.

\section{Speech Title 3 \\ Application of Selective Internal Radiation Therapy (SIRT)}

David Liu

University of British Columbia, Vancouver, Canada

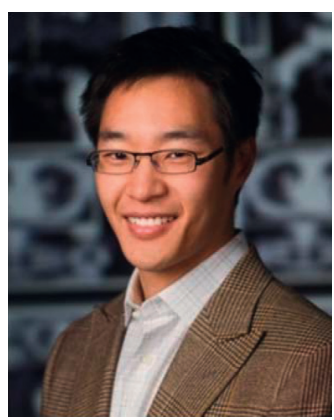

Dr. Liu is a Clinical Associate Professor of Radiology at the University of British Columbia. He maintains an active interventional oncology practice incorporating all aspects of embolization, ablation, venous access, and palliative therapy in all organ systems. Dr. Liu is the co-founder and co-chair of SHOW, and has been credited with 4 book chapters, over 40 publications, and 70 invited lectures, and is an active member and inducted fellow of the Society of Interventional Radiology. He holds specific interest in the treatment of hepatocellular carcinoma and metastatic colorectal carcinoma. Dr. Liu is actively involved in both translational research and clinical trials.

\section{Abstract \\ SIRT Biology and Physics}

SIRT, also known as radioembolization and/or radiomicrosphere therapy, uses $90 \mathrm{Y}$ as a form of brachytherapy whereby microspheres loaded with $90 \mathrm{Y}$ are permanently 
implanted in the target tumor vascular bed within the liver following intra-arterial administration. SIRT aims to selectively delivery radiation to liver tumors whilst limiting the dose delivered to normal liver parenchyma through exploitation of the neovascularization of hepatic primary tumors, since their blood supply preferentially arises from the hepatic arteries as opposed to the portal veins. This preferential supply allows the deposition of $90 \mathrm{Y}$ microspheres delivered through the hepatic artery to preferentially implant within liver tumors in a 3:1 ratio as compared with the 20:1 ratio in normal liver parenchyma. Although whole liver radiotherapy treatment is not well tolerated, radiotherapy to smaller portions of the liver is tolerated without significant complications provided a sufficient amount of normal liver is spared. Treatment with 90Y can be delivered as a resin (SIR-Spheres; Sirtex, Australia) or as glass microspheres (TheraSpheres; MDS Nordion, Canada). As 90Y decays, it emits high energy $\beta$-radiation $(0.97 \mathrm{mEv})$ within the tumor capillary bed, thereby delivering tumoricidal doses of radiation (100-1000 Gy) over a limited range (approximately $2.5 \mathrm{~mm}$ of tissue penetration) for a limited time (90Y decays to $90 \mathrm{Z}$ (zirconium-90) with a half-life of $64.2 \mathrm{~h}$ ). The microparticle size used for SIRT are on average between $20-60 \mu \mathrm{m}$, which is small enough to allow them to enter the vascular network of the tumor, but too big to pass through the capillary bed into the venous circulation.

\section{Mechanism of Action of SIRT}

The mechanism of action of SIRT differs from chemoembolization. Chemoembolization relies on the occlusion of vessels feeding the tumor, thus allowing stasis and hence maximum exposure of the chemotherapeutic agent to the ischemic environment created within the tumor. In contrast, blood flow into the tumor is required for SIRT to allow free oxygen radical generation via the ionization of water molecules from the emitted $\beta$-radiation. This, in turn, results in damage to DNA and activation of apoptosis via an increase in the ratio of BAX:BCL-2 gene expression. Cancer cells are more susceptible to oxidative stress due to their relatively low concentration of superoxide dismutase (SOD) and their increased production of superoxide $\left({ }^{\circ} \mathrm{O}_{2}{ }^{-}\right)$from their higher aerobic metabolism. SOD is an antioxidant which quenches ${ }^{\circ} \mathrm{O}_{2}{ }^{-}$before it can react with hydroxyl radicals $\left(\mathrm{OH}^{*}\right)$ to produce hydrogen peroxide $\left(\mathrm{H}_{2} \mathrm{O}_{2}\right)$. Direct double strand DNA breaks also account for a significant proportion of cellular death. As such, the resin and glass microspheres used for SIRT are small enough to have little or no embolic effect on the medium-to-small sized hepatic arteries, thereby maintaining adequate tissue oxygenation.

\section{Current Evidence}

Significant insights were gained through the retrospective analysis that was conducted by the European Network on Radioembolization with Y90 (ENRY), providing comprehensive analysis from multiple institutions of the cohort of 325 patients. As the population represented the full spectrum of European-based hepatocellular carcinoma, subset analysis revealed an acceptable rate of toxicity associated with nontargeted embolization, and radiation-induced liver disease.
Furthermore, overall gastrointestinal toxicities, and systemic toxicities were within acceptable range to perform the procedure on an outpatient basis. A large number of these patients underwent whole liver single session therapy and did not require hospitalization.

Analysis of at risk and advanced patient populations revealed the following median survival is based on Barcelona score (BCLC) A: $24.4 \mathrm{~m}, \mathrm{~B}: 16.9 \mathrm{~m}, \mathrm{C}: 10.0 \mathrm{~m}, \mathrm{D}: 5.2 \mathrm{~m}$. This data suggested that patients presenting with Barcelona being or greater would experience survival on par with the title the chemoembolization. Further analysis of this population revealed CPA survival estimated at 14.9 months, and CPB at 10.0 months, both favorable outcomes.

As expected, survival in patients with portal venous occlusion secondary tumor thrombus was dependent upon the degree of thrombus. Patients undergoing Y 90 with Peyton portal veins had an aggregate median survival of 15.3 months, while branch occlusion, a meeting occlusion resulted in decreased survival at 10.7 months, and 9.7 months respectively.

Data arising from prospective AHCC05 trial demonstrated very encouraging results for the use and incorporation of SIRT with systemic therapy in an Asian population, demonstrating median survival in excess of 20 months in BCLC B, and $8.2 \mathrm{~m}$ in BCLC C.

Ultimately it has been the experience of the author's group that for patients with good performance status, and healthy liver parenchyma in non-advanced disease state [BCLC A], the clear benefit of SIRT is in the outpatient-based single session nature of its treatment, with minimal post embolization syndrome, and no requirement for hospitalization. Patients that are indicated for downstage to surgical resection or transplantation also benefit from the profound necrosis associated with radiation-induced tumor kill. In addition, patient populations that were traditionally contraindicated for chemoembolization due to advanced disease states [older patients, concerns over cardiac or renal toxicity, portal vein thrombosis, and lower performance status] tolerate the procedure well, with acceptable outcomes.

\section{Consensus Guidelines}

A number of consensus guidelines have established the use of SIRT in specific instances of HCC, including the European Society of Medical Oncology (ESMO), The National Comprehensive Cancer Network (NCCN), the Canadian Provinces of British Columbia and Alberta, and the American Society of Clinical Oncology.

\section{Clinical Studies}

Several major phase 3 clinical trials will address the role of radio embolization with reference to systemic therapies such as sorafenib. The ongoing recruitment of Phase III trials in HCC include SIRveNIB (sorafenib vs SIRT) in Asia, SARAH (sorafenib vs SIRT) in France, and SORAMIC (Sorafenib vs SIRT+sorafenib) in the EU, YES-PVT (SIRT in PVT), STOP-HCC (SIRT vs Sorafenib) will provide strong Level I evidence for broad based incorporation of SIRT in the HCC therapeutic paradigm. 
Satellite 2

\section{Biliary Tract Cancer}

13:45-14:55, July 11, 2014 (Song Bo Room)

Chair

\section{Yasuni Nakanuma}

Professor of Emeritus, Department of Pathology, Shizuoka

Cancer Center, Shizuoka, Japan

Department of Human Pathology,

Kanazawa University Graduate School of

Medicine, Kanazawa, Japan

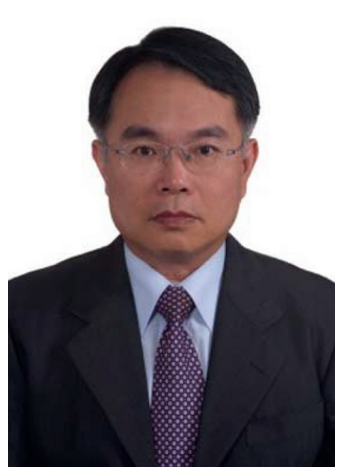

Chair

Jen-Shi Chen

Associate Professor,

Division of Hematology-Oncology,

Department of Internal Medicine,

Linkou Chang Gung Memorial

Hospital and Chang Gung

University College of Medicine,

Taoyuan, Taiwan

\section{Speech Title 1 \\ Diagnosis and Staging of Biliary Tract Cancer \\ Yasuni Nakanuma \\ Department of Pathology, Shizuoka Cancer Center, Shizuoka, Japan; Department of Human Pathology, Kanazawa University Graduate School of Medicine, Kanazawa, Japan}

Prof. Nakanuma is currently Professor Emeritus at Kanazawa University Graduate School of Medicine and Councilor at Department of Pathology, Shiuzoka Cancer Center, Shizuoka. His research interest is hepatobiliary pathology, including benign and malignant diseases. Prof. Nakanuma has won many academic prizes for his scientific achievement, including the Clemens von Pirquet Award (University of California, Davis, USA, 1999), the Oda Award (Annual best Award of Japanese Hepatological Society, 2004), the Japan Pathology Award (Annual best Award of Japanese Association of Pathologists, 2004), and the Robert S. Totten Visiting Professor Award (Pittsburg University, 2013).

\section{Abstract}

Biliary tract cancer remains an intractable malignancy and is clinically detectable at the advanced stage in a majority of cases. Histologically, this carcinoma shows usually tubular and/micropapillary adenocarcinoma with variable fibrous stroma. Recently, two types of pre-neoplastic or early neoplastic lesions of bile duct carcinoma have been proposed in WHO 2010 tumor classification: biliary intraepithelial neoplasm (BilIN) and intraductal papillary neoplasm of bile duct (IPN-B). BilIN is a flat lesion and recognizable microscopically, and is graded into BilIN-1 (low grade), BilIN-2 (intermediate grade), and BilIN-3 (high grade) according to the atypia. BilIN is frequently found in the bile ducts around carcinoma and also in chronic biliary diseases such hepatolithiasis which is occasionally associated with cholangiocarcinoma. BillN-3 is regarded to be followed by invasion of carcinoma into the surrounding tissue. IPN-B is a grossly visible intraductal papillary lesion in variably dilated bile duct lumen and is histologically graded into low, intermediate and high grade according to their atypia, and show four phenotypes (pancreatobiliary, gastric, intestinal and oncocytic). IPN-B of high grade is associated with an invasion into the surrounding tissue. IPN-B occurs in an apparently normal bile duct and also in chronic biliary diseases such as hepatolithiasis and liver fluke infection. In this symposium, pathological diagnosis and staging of biliary tract cancer with an emphasis on pre-and early invasive lesions will be presented.

\section{Speech Title 2 Systemic Treatment of Biliary Tract Cancer Do-Youn Oh}

Division of Medical Oncology, Department of Internal Medicine, Seoul National University Hospital, Seoul, Korea

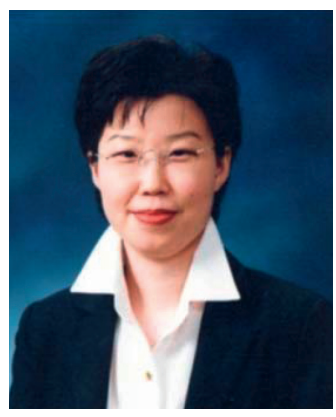


The 5th Asia-Pacific Primary Liver Cancer Expert Meeting (APPLE 2014)

Dr. Oh is currently an Associate Professor in Division of Medical Oncology, Department of Internal Medicine, Seoul National University Hospital. She graduated from Seoul National University College of Medicine in 1997, and got her Ph.D Degree in the Department of Internal Medicine, Seoul National University College of Medicine in 2005. Dr. Oh has lots of committee memberships in the medical society, including Korean Cancer Association, Korean Association of Clinical Oncology, Korean Cancer Study Group, American Society ofClinical Oncology and American Association for Cancer Research. Her main research interest is gastric cancer, pancreatic cancer and biliary tract cancer with particular interest in the translational research and early drug development. She has published over 100 peer-reviewed journal articles.

\section{Abstract}

Systemic chemotherapy is the main treatment modality in advanced biliary tract cancer. During past two decades, the most commonly used cytotoxic chemotherapeutics have been gemcitabine, 5-FU and platinum including cisplatin and oxaliplatin. Gemcitabine and 5-FU have not been compared with each other in terms of efficacy and toxicity in biliary tract cancer. However, with the advent of ABS-02 trial result in 2010, the gemcitabine/cisplatin combination is the most highly recognized current regimen in biliary tract cancer. Biliary tract cancer is a heterogeneous group not only in the primary location but also in molecular characteristics. Somatic alterations in the KRAS, TP53, CDKN2A, SMAD4, BAP1, ARID1A, $P B R M 1$ and $I D H 1 / 2$ have been reported. Targeting those molecular alterations are now being tried in biliary tract cancer. The early phase III trial comparing gemcitabine/oxaliplatin/erlotinib with gemcitabine/oxaliplatin in unselected population failed to show the benefit of erlotinib. Similar design of randomized phase II trial comparing gemcitabine/ oxaliplatin/cetuximab with gemcitabine/oxaliplatin also showed the negative result in terms of overall survival in unselected population. The strategy of adding targeted agent on cytotoxic chemotherapy without selection of optimal target population is not good enough. Biomarker-driven clinical trial is one of the go-throughs. Such strategy has been already initiated in biliary tract cancer. In biomarker-driven trial of panitumumab and chemotherapy combination, only KRAS wild-type patients were selected for the inclusion. This suggests that biomarker-driven trial is feasible in biliary tract cancer, too. Still, the clinical outcomes of systemic treatment in biliary tract cancer are unsatisfactory. Therefore, there is a huge unmet medical needs in this field. The bright side of this is that the opportunity for new drug development is also enormous in this field.

\section{Speech Title 3 \\ Marker-Driven Treatment of BTC: Future Prospective}

Li-Tzong Chen

National Institute of Cancer Research, National Health Research Institutes, Miaoli, Taiwan

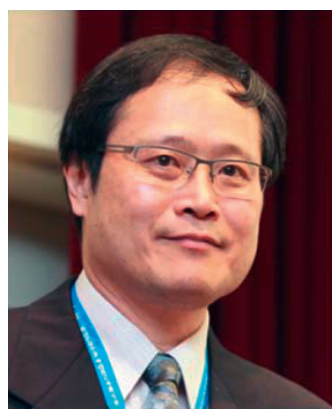

Prof. Chen received his medical training and completed his residency and gastrointestinal fellow trainings at the Kaohsiung Medical University, Kaohsiung, Taiwan. He then participated the first Medical Oncology Training Program, Academia Sinica in 1988-90. He joined National Institute of Cancer Research, National Health Research Institutes (NHRI), Taiwan in 1995. He is now the Acting Director, National Institute of Cancer Research, NHRI, and holds adjunct professorship in several universities in Taiwan. Prof. Chen is a qualified medical oncologist as well as gastroenterologist, whose research interests focus on experimental therapy to develop optimal treatment strategies for gastrointestinal cancers, including hepatocellular carcinoma, biliary tract cancer, pancreatic cancer, and mucosa-associated lymphoid tissue (MALT)-related lymphoma.

\section{Abstract}

Biliary tract cancer (BTC) accounts for approximately $3 \%$ of all gastrointestinal malignancies and has dismal clinical outcome with 5-year survival of less than 5\%. The overall incidence of BTC appears to increasing over the last 3 decade, notably intrahepatic cholangiocarcinoma. Standard chemotherapy for advanced diseased was not really established until very recently. In a phase III study of ABC-02 (Valle et al, NEJM 2010) and a phase II trial of BT22 (Okusaka et al, BJC 2010), patients received gemcitabine and cisplatin (GC) combination had superior overall survival and progression-free survival than those with gemcitabine alone, 11.6 months vs. 8.0 months; hazard ratio $=0.65$; $95 \% \mathrm{CI}$, $0.54-0.78 ; P<0.001$, and 8.8 months vs. 6.7 months; hazard ratio $=0.64 ; 95 \% \mathrm{CI}, 0.53-0.76 ; P<0.001$, respectively, in meta-analysis (Valle et al, Ann Oncol 2014). Recently, Japanese investigators also demonstrated the superiority of gemcitabine plus S-1 (GS) over S-1 in a randomized phase II trial, JCOG0805, with a median OS of 12.5 and 9.0 months, respectively (Morizane et al. 2013 Cancer Sci), which led to an 
The 5th Asia-Pacific Primary Liver Cancer Expert Meeting (APPLE 2014)

ongoing phase III trial comparing GS with GC in advanced BTC (ABTC).

To further improve the therapeutic efficacies of chemotherapy in ABTC, the strategy of inhibiting EGFR in combination with chemotherapy has also been tested in 3 randomized trials for ABTC. Of our own T1210 study (Chen et al, ASCO 2013), the 16.7\% ORR, 4.1 months PFS and 9.8 months OS of patients with GEMOX (gemcitabine/ oxaliplatin) were almost identical to those of a Korean phase III study (Lee et al, Lancet Oncol 2012), and largely compatible with BINGO trial (Malka et al, ASCO 2012). Of intent-to-treat analysis, all three studies showed that adding cetuximab was associated with borderline improvement in PFS but not OS. Of the 122 patients in T1210 study, the preplanned subgroup analysis supposed KRAS mutation was a marginal negative prognostic factor for IIT population, however, it did but could not preclude the potential benefit of adding cetuximab to GEMOX in ABTC (Chen et al, ASCO 2013). In our T1210 population, we then further investigated the predictive and prognostic significance of ROS1, ALK and c-MET, which are negative predictors for the response of lung adenocarcinoma to EGFR TKI and potentially druggable targets. Of the 110 patients, with available tissue for all 3 markers evaluation, 18 (16.3\%) were found to overexpress one of ROS1, ALK or c-MET (RAM) proteins by immunohistochemistry (IHC). All 18 patients had primary IHCC. Of the IHCC subpopulation $(\mathrm{N}=80)$, patients with high RAM expressing tumors $\left(\mathrm{RAM}^{\text {high }}, \mathrm{N}=18\right.$ ) had significant inferior OS than those with low RAM expressing tumors (RAM ${ }^{\text {low }}, \mathrm{N}=62$ ), with a median OS of 5.7 (3.7-9.9) months vs $11.8(9.8-14.5)$ months $(\mathrm{p}=0.018)$. Of the RAM ${ }^{\text {low }}$ IHCC subpopulation, cetuximab-GEMOX treatment was associated with significantly better ORR $(39.3 \%$ vs $14.7 \%, \mathrm{p}=$ $0.041)$, durable DCR (67.9\% vs 38.2\%, p = 0.024) and PFS (median, 7.3 vs 5.2 months, $\mathrm{p}=0.031$ ), and marginally longer OS (median, 13.0 vs 9.8 months, $\mathrm{p}=0.107$ ) than those with GEMOX alone. On the other hand, adding cetuximab did not significantly affect the durable DCR, PFS and OS of patients with RAM ${ }^{\text {high }}$ tumor and GEMOX treatment. Accordingly, we proposed that patients with RAM ${ }^{\text {high }}$ IHCC could have ALK or c-MET inhibitor as the first-line treatment; while patients with RAM ${ }^{\text {low }}$ IHCC should be included into randomized trial to validate the benefit of EGFR antagonists for IHCC. 


\title{
Satellite 3
}

\section{Biomarkers and Drug Development}

\author{
15:15-16:25, July 11, 2014 (Song Bo Room)
}

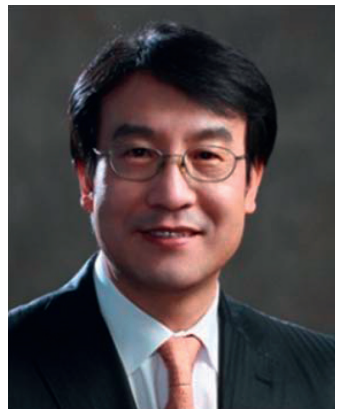

Chair

Ho Yeong Lim

Professor,

Division of Hematology-Oncology,

Department of Medicine, Samsung

Medical Center, Sungkyunkwan

University School of Medicine,

Seoul, Korea

\section{Speech Title 1 \\ Marker-Driven Treatment of HCC: Present Status and Future Prospective}

Ho Yeong Lim

Division of Hematology-Oncology, Department of Medicine, Samsung Medical Center, Sungkyunkwan University School of Medicine, Seoul, Korea

Prof. Lim is a professor of Hematology-Oncology, Samsung Comprehensive Cancer Center, Samsung Medical Center, Sungkyunkwan University, School of Medicine, Seoul, Korea. From 1993 to 2006, he worked as a director of medical oncology at Ajou University, Suwon, Korea. Since 2006, he is a professor of medicine, Samsung Medical Center, Sungkyunkwan University, Seoul, Korea, and, from 2009 to 2011 he was appointed Head of the Division of Hematology-Oncology at Samsung Medical Center. Prof. Lim is a member of numerous societies including: the Korean Association for Clinical Oncology, the Korean Cancer Association, the Korean Association of Internal Medicine, the Korean Medical Association, the Korean Cancer Study Group, the American Association of Cancer Research and the American Society of Clinical Oncology. Prof Lim has published extensively in local and international journals and has been principle investigator in over 90 clinical trials.

\section{Abstract}

Hepatocellular carcinoma (HCC) currently represents the fifth most frequently diagnosed cancer worldwide but the second most frequent cause of cancer death, and is highly prevalent in Asia. In Korea, HCC accounts for approximately $10 \%$ of all malignancies and is one of the leading causes of cancer death.

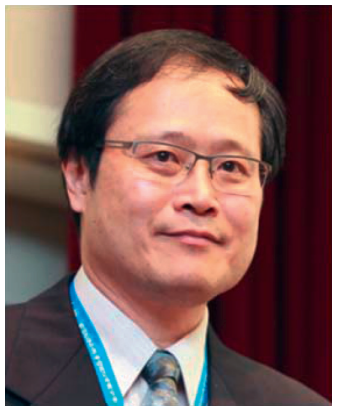

\section{Chair \\ Li-Tzong Chen}

Professor and Director, National Institute of Cancer Research, National Health Research Institutes, Miaoli, Taiwan
With recent advances in understanding of the molecular mechanisms of hepatocarcinogenesis, molecular targeted therapy has offered new prospects and attracted a great deal of attention with regard to its use in the standardized treatment of HCC. Systemic treatment with sorafenib, a multikinase inhibitor targeting Raf kinase and receptor tyrosine kinases (RTKs) including platelet-derived growth factor receptor (PDGFR), vascular endothelial growth factor receptor (VEGFR), and c-kit (a receptor specific for stem cell factor), is recommended for patients with advanced HCC. Sorafenib is currently the first and so far the only drug that has shown survival advantage in HCC patients.

However, sorafenib provides a modest clinical efficacy with low objective response and short duration of disease stabilization. For improving of treatment outcome with sorafenib and other promising drugs, it is important to understand the key oncogenic drivers and the resistance mechanisms to drugs in HCC. In addition, the translational research to find predictive markers is required to facilitate the selection of patients who are most likely to derive benefit from molecular targeted agents. Unfortunately, the investigations to date did not find any known reliable predictive markers to identify patients who can have benefit from sorafenib or other targeted agents.

The investigation for strategy to improve clinical outcome beyond sorafenib in HCC remains an urgent unmet medical need, therefore we need continuing research to identify key molecular targets and reliable biomarkers to overcome this challenge. Predictive markers or specific markers relevant to mechanism of drug could provide more enhanced treatment option to selected patients and this strategy could significantly improve the clinical efficacy in HCC. Recently, several clinical trials are ongoing to identify survival difference according to the presence of certain markers. 


\section{References}

1. LLovet JM, Ricci S, Mazzaferro V, et al: Sorafenib in advanced hepatocellular carcinoma. N Engl J Med 2008;359:378.

2. Cheng AL, Kang YK, Chen Z, et al: Efficacy and safety of sorafenib in patients in the Asia-Pacific region with advanced hepatocellular carcinoma: a phase III randomized, double blind, placebo-controlled trial. Lancet Oncol 2009;10:25.

3. Zhu AX: Molecularly targeted therapy for advanced hepatocellular carcinoma in 2012: current status and future perspectives. Semin Oncol 2012;39:493.

4. Santoro A, Rimassa L, Borbath I, et al: Tivantinib for secondline treatment of advanced hepatocellular carcinoma: a randomized, placebo-controlled ohase 2 study. Lancet Oncol 2013;14:55.

5. Lim HY, Yen C-J, Tak W-Y, et al: A phase II trial of MEK inhibitor BAY 86-9766 in combination with sorafenib as first-line systemic treatment for patients with unresectable hepatocellular carcinoma (HCC). J Clin Oncol 2012; 30(suppl):abstr 4103).

6. Zhu AX, Gold PJ, El-Khoueiry AB, et al: First-in-man phase 1 study of GC33, a novel recombinant humanized antibody against glypican-3, in patients with advanced hepatocellular carcinoma. Clin Cancer Res 2013;19(4):920.

\section{Speech Title 2 \\ Biomarker for Hepatocellular Carcinoma: Current Status and Future Perspectives}

\section{Wei Tang}

Hepato-Biliary-Pancreatic Surgery Division, Department of Surgery, Graduate School of Medicine, the University of Tokyo, Tokyo, Japan

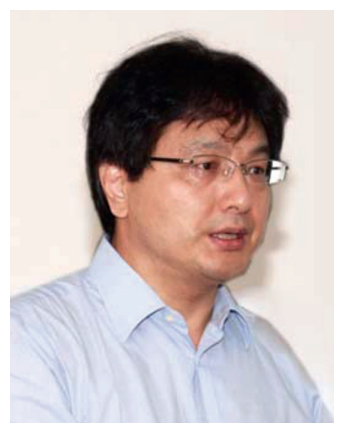

Dr. Tang is currently an assistant professor at Department of Surgery, The University of Tokyo Hospital and holds adjunct professorship in several universities in China. He has published more than 140 English papers until now. His research interests include intrahepatic invasion and metastasis of HCC, gastroenterological cancer, HCC, clinical pathology, metastasis, invasion, angiogenesis, chemotherapy, cancer drug targets, glycoprotein and tumor maker. In the research field of tumor marker for HCC, he devotes to promoting the Japan-China joint research projects in recent years, and has published a number of papers in this field. Dr. Tang is Associate Editor-in-Chief ofJournal of Pathogen
Biology and Executive Editor of several journals, including BioScience Trends, Drug Discoveries \& Therapeutics, and Intractable \& Rare Diseases Research.

\section{Abstract}

Hepatocellular carcinoma (HCC) is one of the most common liver neoplasms in the world, with an estimated global incidence of 782,000 new cases in 2012. It is prevalent in Asia and Africa, and its prevalence is increasing in the America, Canada and Europe. The molecular pathogenesis of HCC is a complex multistep process in which many signaling cascades are altered, leading to a heterogeneous molecular profile. The management of HCC including screening, diagnosis, therapy and recurrence prevention remains a lot of challenges. Current means of HCC screening or diagnosis mainly depend on the tumor imaging techniques, especially the ultrasonography. Surgical resection and liver transplantation are most promising therapies which offer the best therapeutic outcome of treating HCC with a 5-year overall survival more than $50 \%$, however they are only feasible for early and interim-stage. Though there is diversity between the eastern and western countries in the application of serum biomarkers, it always plays a supporting role for the radiological modalities in the current clinical study of HCC. Alpha-fetoprotein (AFP) is the first and foremost used biomarker for HCC detection and follow-up. The sensitivity and specificity of AFP vary widely; up to $50 \%$ of patients with HCC represent a serum AFP level less than $20 \mathrm{ng} / \mathrm{mL}$, on the contrary AFP elevation might also be found in patients with non-malignant chronic liver disease, such as liver cirrhosis. Since some new biomarkers in the circulation system has been discovered due to the increase of understanding of cancer biology and technological advances, the era that AFP is considered as the single and standard biomarker of HCC has come to an end. AFP is currently not recommended for screening for HCC-high-risk population and is even abandoned from the diagnostic guideline of American Association for the Study of Liver Disease (AASLD). These newly discovered biomarkers can be roughly divided into the following categories: oncofetal and glycoprotein antigens (including AFP-L3, GPC3); enzymes and isoenzymes (including DCP, GGT, AFU, Human Carbonyl Reductase 2. et al); growth factors and their receptors (including TGF-Beta, TSGF, EGFR, HGF/SF, et al); molecular markers (mRNAs) and pathological biomarkers. This study is to overview current status of these novel biomarkers of HCC, their clinical application and the potential use in the future. We found that most of the conclusions of biomarkers are drawn by the retrospective studies. The current study and application of biomarkers is far from comprehensive. These novel biomarkers of HCC could not only be applied in screening but also be potentially helpful in optimize modality, prediction of prognosis or recurrence and leading to novel targets for therapeutic interventions in near future. The potential and reliability of serum biomarkers needs more fine-designed evaluation trials. 
The 5th Asia-Pacific Primary Liver Cancer Expert Meeting (APPLE 2014)

\section{Speech Title 3 \\ Circulating Tumor Cells}

Jian Zhou

Liver Cancer Institute and Department of Liver Surgery, Zhongshan Hospital, Fudan University, Shanghai, China

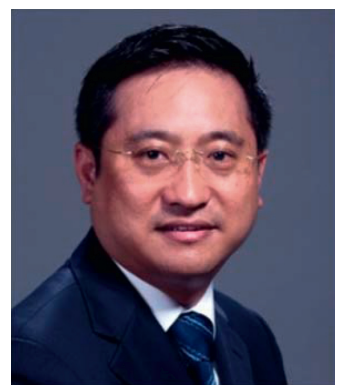

As the Professor and Director of Liver Surgery Department, Prof. Zhou has experienced more than 6,000 cases of hepatectomy for liver cancer patients and more than 1,000 cases of orthotopic liver transplantation. His research focused on early diagnosis, prevention and treatment for recurrence and metastasis of hepatocellular carcinoma. As PI, Prof. Zhou has 16 research programs including "China National Funds for Distinguished Young", "National High Technology Research and Development Program of China (863 program)", "National Natural Science Foundation of China" etc., regarding hepatocellular carcinoma in the fields of early diagnosis, mechanism exploring of recurrence and metastasis, and translational medicine. He has published 55 SCI papers as the first or correspondence author in journals including Journal of Clinical Oncology, Hepatology, Gastroenterology, Cancer Research, and Clinical Cancer Research.

\section{Abstract}

The spread of circulating tumor cells (CTCs) in the blood plays a major role in the initiation of metastases and tumor recurrence after surgery. In our previous work, we confirmed that EpCAM+ HCC cells derived from cell lines and tumor specimens were highly invasive and tumorigenic, and EpCAM could serve as a biomarker for tumor-initiating cells in HCC. Thus, detection of CTCs by EpCAM expression may indicate the more aggressive stem cell-like CTCs in HCC.

Blood samples from 123 HCC patients were tested by CellSearch system prior to resection and 1 month thereafter. The EpCAM+ CTCs were present in $66.67 \%$ of patients, and the cell count measured in $7.5 \mathrm{ml}$ of blood (CTC7.5) ranged between $1-34$. Fifty-one patients had CTC7.5 of $\geq 2$ preoperatively, and these individuals developed tumor recurrence earlier than those with CTC7.5 of $<2$ CTCs. A preoperative CTC7.5 of $\geq 2$ was an independent prognostic factor for tumor recurrence. Its prognostic significance also applied to patients with alpha-fetoprotein (AFP) levels of $<400 \mathrm{ng} / \mathrm{ml}$ or subgroups with low recurrence risk. Significant decrease of CTC-positive rates $(66.67 \%$ to $28.15 \%, \mathrm{P}<0.05)$ and CTC7.5 values $(2.60 \pm 0.43$ to $1.00 \pm 0.36, \mathrm{P}<0.05)$ was observed 1 month after resection. Patients with consistent CTC7.5 $<2$ had lower recurrence rates than those with values consistently $\geq 2$ (15.5\% vs. 87.50\%, P < 0.001). The EpCAM+ CTCs displayed cancer stem cell biomarkers (CD133 and ABCG2), epithelialmesenchymal transition, Wnt pathway activation, high tumorigenic potential and low apoptotic propensity.

We further investigated the epithelial-mesenchymal transition (EMT) status and clinical significance of CTCs at different vascular sites to gain insights into the metastatic route and the dynamic changes of CTC biologic features during their trafficking. CTCs and CTMs in suprahepatic inferior vena cava (SHIVC) might be readily trapped in the lung initiating metastatic lesions, while CTCs and CTMs detected in peripheral circulation re-seeded to remnant liver causing local recurrence. Because all CTMs released directly from primary HCC were filtered by lung, the CTMs in PV might be the product of spontaneous re-clustering of individual CTCs. Moreover, the abundance of epithelial CTCs in SHIVC and mesenchymal CTCs in PV implicated that CTCs dynamically toggled EMT status during dissemination, potentiating their migratory capability.

Compared with the CellSearch system, CTC detection by qRT-PCR has the advantage of improved sensitivity, versatility, and cost-effectiveness. Therefore, we further constructed a novel optimized negative enrichment and qRT-PCR-based CTC detection platform that exhibits high sensitivity, specificity, and reproducibility with a small sample volume requirement for HCC patients.

Pretreatment EpCAM mRNA-positive (EpCAMmRNA+) CTC level showed prognostic significance in HCC patients treated with resection, TACE, and radiotherapy (all $\mathrm{P}<0.050$ ). Most of the patients showed a decrease in CTC levels after treatment that reflected tumor response. In contrast, patients with an increased CTC level showed disease progression after treatment. Detection of EpCAMmRNA+ CTC by our qRT-PCR platform might serve as a novel indicator for the risk of recurrence or progression in patients with HCC and a promising surrogate tool for surveillance of the therapeutic response instead of radiologic scans with the benefits of increased convenience and safety. 


\title{
Satellite 4
}

\section{Bridging Clinical and Histological Diagnosis of HCC}

\author{
15:15-16:25, July 12, 2014 (Song Bo Room)
}

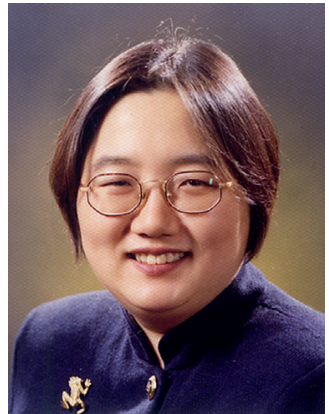

Chair

Young Nyun Park

Distinguished Professor,

Department of Pathology, Yonsei

University College of Medicine,

Seoul, Korea

\section{Speech Title 1 \\ Histological Diagnosis of HCC: Inquiry from the Clinic}

Chien-Hung Chen

Department of Internal Medicine, National Taiwan University Hospital, Taipei, Taiwan

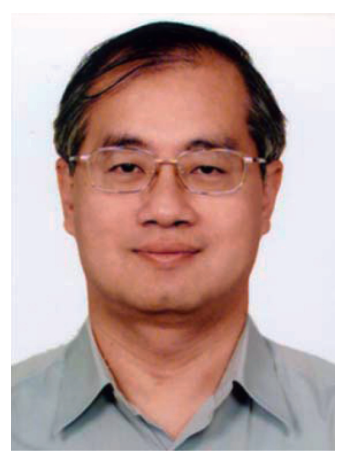

Dr.ChengothisM.D.degreefrom CollegeofMedicine, National Taiwan University, Taipei, Taiwan in 1987. He got his Ph.D. in the same University in 2001. He received his resident and fellow training in Department of Internal Medicine, National Taiwan University Hospital. He also spent two years (1997 1999) as PostDoctor Fellow in Department of Oncology, School of Medicine, Johns Hopkins University. He is now an Associate Professor of Internal Medicine, College of Medicine, National Taiwan University and a Staff physician, Department of Internal Medicine, National Taiwan University Hospital. Dr. Chen's research interest includes clinicalstudies in human hepatocellular carcinoma, proteomics in human hepatocellular carcinoma, and hepatic immunology.

\section{Abstract}

Hepatocellular carcinoma (HCC) is probably the only one malignancy that diagnosis could be made without tissue proof.

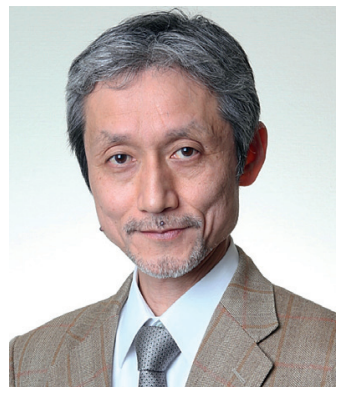

Chair

Michiie Sakamoto

Professor,

Department of Pathology, Keio University School of Medicine,

Tokyo, Japan
American Association for the Study of Liver Diseases (AASLD) and European Association for the Study of the Liver (EASL) guidelines suggest that HCC could be diagnosed by one or two imaging technique (CT or MRI) showing the HCC radiological hallmark if the hepatic tumor is larger than $1 \mathrm{~cm}$ in the cirrhotic background. Biopsy is reserved for tumors without typical images characteristics of HCC. In Asian Pacific Association for the Study of the Liver (APASL) consensus recommendations, there is limitation of tumor size by imaging diagnosis. APASL consensus does not mention the role of liver biopsy in the diagnosis of HCC. Because of the recent advances in the molecular therapy, it is emphasized that tumor tissues are necessary to help us understand the molecular pathways of cancers. This might be important in the future personalized therapy for HCC. Some patients are not suitable for tumor biopsy because of severe coagulopathy or other contraindication for liver biopsy. The risks of liver biopsy must be balanced against the possible benefits. For those with non-diagnostic images studies, liver biopsy is indicated. However, differentiation between welldifferentiated HCC vs. dysplasia is not always easy. Several molecular biomarkers have been proposed for the differentiation. But these biomarkers are far from perfect. Taken together, it is still in debate whether routine tumor biopsy should be done to all patients with suspicious HCC.

\section{Speech Title 2 \\ Mixed Hepatobiliary Carcinoma: Pathological and Clinical Features \\ Young Nyun Park \\ Distinguished Professor, Department of Pathology, Yonsei University College of Medicine, Seoul, Korea}

Prof. Park is a professor in the Department of Pathology at Yonsei University College of Medicine, in 2006. She was appointed to the Vice Dean for Graduate Affairs of Yonsei 
The 5th Asia-Pacific Primary Liver Cancer Expert Meeting (APPLE 2014)

University College of Medicine since 2008. Prof Park has focused on understanding the early stage of hepatocarcinogenesis and cancer stem cells of hepatocellular carcinoma. She has published about 160 peer reviewed papers in international journals, including Hepatology, Journal of Hepatology, American Journal of Pathology, etc. She has contributed to the sections of hepatocellular carcinoma and combined hepatocellular-cholangiocarcinoma in the 4th edition of WHO Classification of Tumours of the Digestive System in 2010 and the textbook entitled Practical Hepatic Pathology (Elsevier, 2011). She has received 16 awards, including the best research awards from the Korean Society of Pathologists in 2006, the Korean Association for the Study of the Liver in 2002, Seoul Medical Association in 2013, and Yonsei University in 2010, 2011, 2013 and 2014.

\section{Abstract}

Cancer cells in hepatocellular carcinoma (HCC) are heterogeneous, and cancer cells exhibiting stem cell-like features ("cancer stem cells") can be found in HCCs. Recent studies have introduced a new subtype of HCC, which shows a fraction of tumor cells (> 5\%) expressing stem/progenitor cell markers but is otherwise not recognizable by routine hematoxylin-eosin stain. HCCs expressing stem/progenitor cell markers reportedly show more frequent vascular invasion, more fibrous stroma, and more aggressive behavior, compared to conventional HCCs without stem/progenitor cell marker expression. Additionally, there are two other entities that exhibit stem cell-like features: The first one is combined hepatocellular-cholangiocarcinoma, classical type that has areas of typical HCC and areas of typical cholangiocarcinoma. Interestingly, some of these tumors may comprise a component with stem cell features in the transitional zone. The second one is combined hepatocellular-cholangiocarcinoma with stem cell features, which is a rare tumor that has been variously described as "hepatic stem cell tumors," "primary liver carcinoma of intermediate (hepatocyte-cholangiocyte) phenotype," and "cholangiolocellular carcinoma." These terms were recently defined to describe, as a whole, combined hepatocellular-cholangiocarcinoma with stem cell features, and were subtyped as typical subtype, intermediate-cell subtype, and cholangiolocellular subtype, respectively, in the 4th edition of the WHO Blue Book. In these tumors, the majority of tumor cells show stem/progenitor-like cells or intermediate features between hepatocytes and cholangiocytes, and there are no or only focal areas of typical HCC and/ or typical cholangiocarcinoma. Accordingly, there is increasing demand for functional classification that can predict the biological behavior of HCC, and new ways to classify HCC using liver cancer stem cell markers are now under investigation. Further studies are required to facilitate this classification.

\section{Speech Title 3 Clinical Diagnosis of HCC: Feedback from Pathologists}

Shiu-Feng Huang

Institute of Molecular and Genomic Medicine, National Health Research Institute, Miaoli, Taiwan

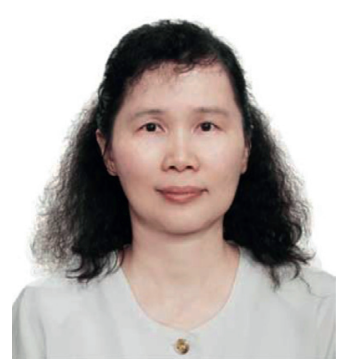

Dr. Huang is an experienced senior pathologist, especially in lung, liver and pediatric pathology. She has up to 188 publications so far. Dr. Huang's research interests are biobank establishment and molecular genetic studies related to tumorigenesis of solid tumors, mainly focusing on hepatocellular carcinoma and lung cancers. She also has expertise in diagnostic molecular pathology, focusing on molecular biomarker research in solid cancers.

\section{Abstract}

The diagnosis of hepatocellular carcinoma, unlike the other human malignancies, a pathology diagnosis by tissue sampling is often not necessary, which is quite unusual and has become a controversial issue. According to the current AASLD guidelines, any hepatic lesion larger than $1 \mathrm{~cm}$ that demonstrate the classical pattern of HCC by image study (CT or MRI) can be deemed as such and treated accordingly without biopsy. This guideline has resulted in a dramatic decrease of liver biopsy for the diagnosis of HCC. Currently, only around $36 \%$ of HCCs in Taiwan have tissue proof. However, even with typical image findings of HCC, it is still not $100 \%$ true. Several examples will be shown. In addition, the histology patterns of cancers often are related to prognosis or treatment response, which is only available by tissue sampling. With the increasing number of targeted therapy for human cancers, identifying the drug targets or its mechanism also often rely on the patient's tumor samples. For example, identification of the EGFR mutation from patient's tumor tissue has become indispensible for the current non-small cell lung cancer treatment. The histology patterns of lung adenocarcinoma also have been correlated with the prognosis, and have become predictors for adjuvant therapy in stage I patients. All of the above conditions may also become true for new anti-HCC drugs in the future. Lack of pathology diagnosis for HCC could be a drawback for future new anticancer drug development. 
The 5th Asia-Pacific Primary Liver Cancer Expert Meeting (APPLE 2014)

\section{Satellite 5 \\ Novel Imaging Modality}

08:00-09:40, July 13, 2014 (Song Bo Room)

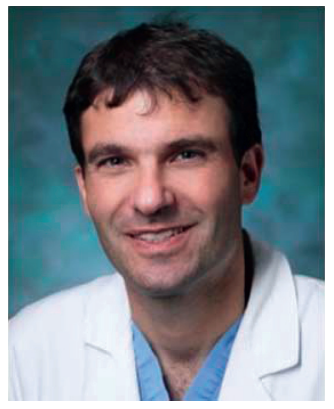

\author{
Chair \\ Jeff Geschwind \\ Professor and Director \\ Johns Hopkins University School \\ of Medicine, Baltimore, USA \\ Interventional Radiology Center, \\ Johns Hopkins Hospital, \\ Baltimore, USA
}

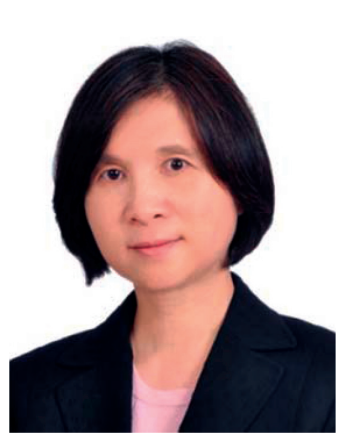

Chair

Li-Ying Liao

Chief,

Department of Gastroenterology,

Taipei City Hospital Renai Branch,

Taipei, Taiwan

\section{Speech Title 1 \\ Assessment of Tumor Response after Loco- Regional Therapy}

Jeff Geschwind

Johns Hopkins University School of Medicine, Baltimore, USA; Interventional Radiology Center, Johns Hopkins Hospital, Baltimore, USA

Dr. Geschwind is currently a Professor of Radiology, Surgery and Oncology at the Johns Hopkins University School of Medicine. He has focused his clinical expertise on treating hepatic cancer and other malignancies and continued to improve current therapies for patients with liver cancer, in particular catheter-based therapies such as chemoembolization and radioembolization. Dr. Geschwind has authored or co-authored more than 370 articles on interventional oncology and specifically the treatment of liver cancer, many of them published in the most prestigious peer-reviewed journals, including Clinical Cancer Research, Journal of Clinical Oncology, and Radiology. He serves as the Consultant to the Editor of the Journal Radiology and is on several Editorial Boards including the main interventional radiology journal Cardiovascular and Interventional Radiology. In 2011, Dr. Geschwind was honored with a "Top Doctor" award from US News and World Report, and he was recognized as one of the "Top Ten Radiologists" in the United States by Medical Imaging Magazine.

\section{Abstract}

Assessing tumor response of HCC lesions after intraarterial therapies is of major clinical interest. Although overall survival (OS) continues to be the ultimate end point of clinical cancer research, most trials rely on imaging biomarkers in order to assess local tumor status as well as to compare the efficacy of different intraarterial modalities. The primary clinical purpose of follow-up imaging, however, remains a reliable and early identification of non-responders in order to then subject these patients to re-treatment. Over the last two decades, tumor response criteria have come a long way from purely size-based, anatomic methods such as the Response Evaluation Criteria in Solid Tumors (RECIST) and the World Health Organization (WHO) guidelines towards more functional, enhancement- and diffusion-based parameters with a strong emphasis on MRI as the ultimate imaging modality. This trend is based on the mechanism of most intraarterial therapies that involve the element of embolization in order to induce tumor infarction which in return, leads to tissue necrosis without immediate effects on the overall lesion size. Because of that, enhancement-based response criteria such as the European Association for the Study of the Liver (EASL) guideline as well as modified (m) RECIST have become increasingly popular and were shown to represent tumor pathology more accurately as compared to RECIST. However, these techniques have considerable limitations because of their limited reproducibility as well as their inability to evaluate entire lesions, which makes them inevitably inaccurate in predicting the true extent of the mostly heterogeneous, post-therapeutic tumor necrosis. The narrow applicability of the above mentioned techniques provided the rationale for the development of new, 3D quantitative assessment techniques for tumor response after transarterial chemoembolization as well as other catheter-based intraarterial therapies. As a first milestone, a goal was to develop and to validate a workflow-efficient tumor segmentation technique which would address the issue of accuracy and clinical practicability. The competing concepts of fully automated tumor segmentation vs. a semi-automated technique were tested and the latter was identified as a more accurate and clinically intuitive approach primarily because it allowed for a 
radiological reader to modify the enclosed liver tumor volume. Because of the 3D quantitative character of this technique, this method has been referred to as volumetric (v) RECIST. The need for new 3D quantitative approaches that would encompass the functional parameters of HCC lesions led to the advent of two additional MR-based parameters, the quantitative (q) EASL as well as the quantitative apparent diffusion coefficient (qADC). Both techniques are anchored in a regionof-interest (ROI)-based voxel-by-voxel analysis and quantification of the brightness signal, which allows for a wholetumor analysis. These techniques provide the radiological reader with a precise quantification of the enhancing or diffusion-restricted tumor portions and were shown to provide an accurate correlation with tumor pathology as seen on tumor explants. Recent studies have also confirmed the intuitive advantages of these techniques over the non-3D methods in predicting survival both, in primary and secondary liver cancer. However, a major drawback continues to be the necessity of dedicated software which would allow for a dissemination of these techniques beyond academic institutions. In summary, new 3D quantitative tumor assessment techniques continue to be a hot topic of clinical research in interventional oncology and the great wealth of knowledge that has been added over the last decade continues to grow. A future development might very well be the inclusion of 3D quantitative tumor analysis techniques into novel staging systems in order to finally achieve the perfect symbiosis between peri-procedural imaging with intra-procedural treatment decisions.

\section{Speech Title 2 \\ Gd-EOB-DTPA Enhanced MRI for Hepatocellular Carcinoma: Correlation with Molecular and Genetic Pathology}

Osamu Matsui

Department of Advanced Medical Imaging, Kanazawa University Graduate School of Medical Sciences, Kanazawa, Japan

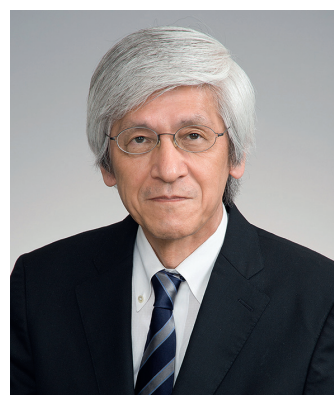

Prof. Matsui was promoted to full professor and chairman for the Department of Radiology, Kanazawa University in 1999.
In 2010, he was appointed for the dean of Kanazawa University Graduate School of Medical Science, and is now the specially appointed research professor of the Department of Advanced Medical Imaging and professor of emeritus at the same institute. His main clinical and research contributions have been in the diagnostic imaging and interventional radiology, especially for liver cancer. He is the author of around 400 original English papers which appeared in Medline. He was the editor-in-chief of Japanese Journal of Radiology which is the official journal of Japan Radiological Society, and now an associate editor of gastrointestinal section of Radiology. He was selected one of the distinguished scientists of Japan in 2009 by the Minister of Education, Culture, Sports, Science and Technology.

\section{Abstract}

Gd-EOB-DTPA enhanced MRI (EOB-MRI) has proved to be extremely accurate in the detection and characterization of HCCs, and the imaging algorithm of HCC is drastically changing now in Japan. To understand the molecular mechanism of EOB-MRI is important for the accurate interpretation of imaging findings and personalized medicine of HCCs. [Correlation between signal intensity of hepatocellular carcinoma (HCC) on hepatobiliary (HB) phase of EOB-MRI and hepatocyte uptake transporter OATP1B3 (OATP8) expression in HCC cells] Our analysis revealed the intense correlation between signal intensity of HCC on hepatobiliary (HB) phase of EOB-MRI and hepatocyte uptake transporter OATP8 (1B3) expression in HCC cells. According to our experiences, there is a pinpoint correlation between hyperintensity on $\mathrm{HB}$ phase of EOB-MRI in hepatic mass lesions and OATP1B3 expression. Therefore, HB phase of EOB-MRI is an extremely sensitive (indirect) molecular imaging for hepatocyte uptake transporter OATP1B3 expression in HCC cells. (Kitao A, Matsui O et al. Radiology 2010; 256:817-26). [Correlation between OATP1B3 expression and signal intensity on HB phase of EOB-MRI in hepatocellular nodules during multi-step hepatocarcinogenesis] Semiquantitative analysis of immunohistochemistry of OATP1B3 expression correlated with tumor differentiation revealed that the expression of OATP1b3 was significantly decreased in accordance with the elevation of the grade of malignancy of the nodules from dysplastic nodule to poorly differentiated HCCs. In addition, OATP1B3 expression decreased in around $80 \%$ of early HCC, resulting in very high detection rate of hypovascular early HCC on HB phase of EOB-MRI. On the other hand, in moderately differentiated HCCs, around $12 \%$ showed equivalent or increased expression probably due to some genetic alteration during hepatocarcinogenesis. (Kitao A, Matsui O, etal. Eur Radiol.2011;21:205666). [Hypervascular classic HCC showing hyperintensity on HB phase of EOB-MRI is biologically less aggressive] $82 \%$ of hypervascular HCCs showing hyperintensity on HB phase were moderately differentiated HCC, remaining 18\% well differentiated HCC and no poorly differentiated HCC. The differentiation grade was higher and portal vein invasion was less frequent in hyperintense HCC than hypointense HCCs. 
The 5th Asia-Pacific Primary Liver Cancer Expert Meeting (APPLE 2014)

The percentage of patients showing normal serum levels of AFP, L3 fraction and PIVKA-II in the hyperintense HCCs were higher than those of hypointense HCCs. The patients with hyperintense HCCs showed a significant lower recurrence rate than those with hypointense HCCs. The patients with hyperintense HCCs tended to show longer survival than those with hypointense HCCs. (Kitao A, Matsui O, et al. Radiology. 2012;265:780-9) Immunohistochemical analysis revealed the mature hepatocyte origin with beta-catenin mutation of hyperintense HCCs. [Hyperintense HCCs shows a peculiar gene and molecule expression] To understand the transcriptomic characteristics of HCCs overexpressing OATP1B3, we analyzed microarray data of HCCs and found that these HCC had a peculiar pattern of overexpressed genes. Genes associated with mature hepatocyte function significantly up-regulated in these HCCs, and transcription factor analysis revealed that HNF4A was most strongly activated. Knockdown of HNF4A in HCC cells showing an uptake of Gd-EOB-DTPA resulted in the loss of OATP1B3 expression. EOB-MRI is considered to be valuable in the non-invasive diagnosis of genetic subtype of HCC and for the personalized medicine. (Yamashita T. Matsui O. Kaneko S. et al. Hepatology in press).

\section{Speech Title 3 Imaging Tumor Angiogenesis Bang-Bin Chen}

Department of Medical Imaging and Department of Radiology, National Taiwan University Hospital, Taipei, Taiwan

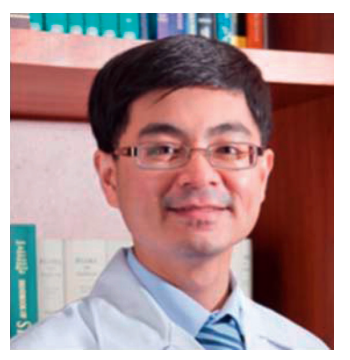

Dr. Chen received residency training in the Department of Radiology, National Taiwan University Hospital, and became attending staff since 2008. I got the master degree of Graduate Institute of Clinical Medicine in National Taiwan University in 2009. His research interests mainly focus on functional imaging, especially dynamic contrast-enhanced MRI in the various diseases, including acute myeloid leukemia, myelodysplastic disease, hepatic fibrosis, hepatocellular carcinoma, and metastatic brain tumors. He is also interested in the application of hepatocyte-specific contrast agent in the evaluation of hepatic tumor and fibrosis.

\section{Abstract}

Dynamic contrast-enhanced magnetic resonance imaging (DCE-MRI) enables tumor vascular physiology to be assessed. Within the tumor tissue, contrast (gadolinium chelated agent) extravasates from intravascular into the extravascular extracellular space (EES), which results in signal enhancement on T1-weighted MRI. These signal intensity - time curves can be deconvoluted by mathematical modeling to extract quantitative parameters that may reflect tumor perfusion, vascular volume, vessel permeability and angiogenesis. Because HCC is a hypervascular tumor, many emerging therapies focused on the inhibition of angiogenesis. DCE-MRI combined with a pharmacokinetic model allows us to produce highly reproducible and reliable parametric maps of quantitative parameters in HCC. Successful therapies change quantitative parameters on DCE-MRI, which may prove to be accurate and early indicators of tumor response to anti-angiogenesis agents. In the setting of clinical trials, dynamic contrast enhanced MR imaging may provide relevant clinical information of the pharmacodynamic effect of novel drugs, including an early indication of biologic effect. However, current DCE-MRI technique lacks standardization across multiple MR platforms and institutions, making it difficult to implement the technique in a multicenter setting. Besides, there is a need to establish clear thresholds for a significant response when using quantitative DCE MR imaging parameters for assessment of therapy response.

Similar to DCE-MRI, Perfusion CT imaging of the liver is performed by acquisition of serial images after contrast bolus injection to obtain various perfusion indices, including regional tumor blood flow, blood volume, flow-extraction product, and permeability-surface area product. Previous reports have suggested that CT perfusion parameters can be used for quantifying tumor vascularity and angiogenesis in HCC, or as biomarkers to monitor response to chemoembolization, chemotherapy and a range of different targeted agents ENREF_40. For example, in one study of locally advanced HCCs receiving bevacizumab and cytotoxic therapy, high pretreatment Ktrans by perfusion CT indicated those patients had a RECIST response. The main drawback associated with perfusion CT was radiation dose, but recent advances in MDCT technology many help achieve acceptable radiation dose in HCC patients. 


\section{Poster Session}

\section{Poster Display Time/Place:}

09:00-16:30, July 11, 2014 (The Grand Ballroom)

09:00-16:30, July 12, 2014 (The Grand Ballroom)

09:00-11:00, July 13, 2014 (The Grand Ballroom)

All the accepted abstracts will be presented in poster format during APPLE 2014. The Scientific Program Committee will select abstracts with special scientific or clinical interest for oral presentation in the Poster Discussion Sessions as follows:

\section{Poster Discussion Session 1}

\section{Screening/Diagnosis/Early-Stage Disease}

10:00-11:55, July 12, 2014 (Song Bo Room)

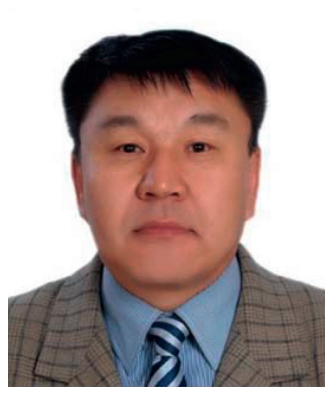

\section{Chair \\ Oidov Baatarkhuu}

Professor and Chairman,

Department of Infectious

Diseases, School of Medicine,

Health Sciences University of

Mongolia, Ulaanbaatar, Mongolia

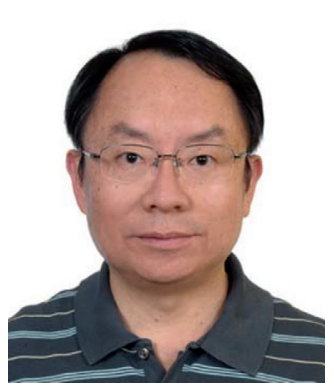

Chair

Rong-Nan Chien

Professor,

Liver Research Unit, Chang Gung

Memorial Hospital and University,

Keelung, Taiwan

\section{Poster Discussion Session 2}

\section{Treatment for Intermediate-Advanced Stage Disease}

13:15-15:00, July 12, 2014 (Song Bo Room)

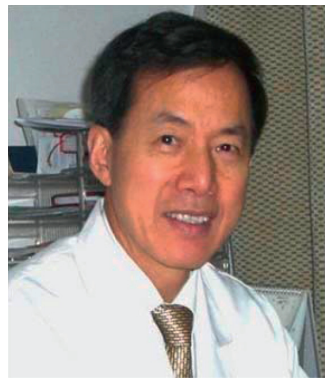

Chair

Ren-Jie Yang

Professor and Director,

Peking University Cancer

Hospital, Beijing, China

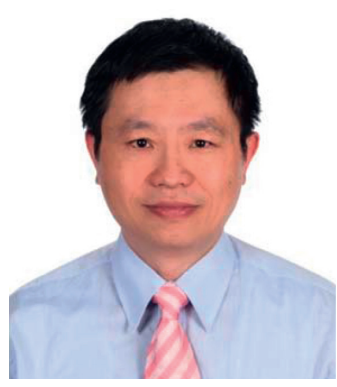

Chair

Ji-Hong Hong

Chair and Professor,

Department of Radiation

Oncology, Chang Gung Memorial

Hospital at Linkou, Taoyuan,

Taiwan 


\section{Category 1 Prevention of HCC}

\begin{abstract}
PS01-001
Inhibitory Effect of Guttiferone K on Tumor Metastasis in Hepatocellular Carcinoma

Kaikai Shen ${ }^{1}$, Zhichao Xi ${ }^{1}$, Hua Wang ${ }^{2}$, Qihan Dong ${ }^{3}$, Hongxi $\mathrm{Xu}^{1 *}$

${ }^{1}$ School of Pharmacy, Shanghai University of Traditional Chinese Medicine, Shanghai, China; ${ }^{2}$ Department of Physiology and Institute of Vascular Medicine, Chinese University of Hong Kong, HongKong, China; ${ }^{3}$ School of Science and Health, the University of Western Sydney, Sydney, Australia
\end{abstract}

Introduction: Hepatocellular carcinoma (HCC) has a high incidence in China, Australia, Europe and the United States. Alarmingly, of all cancers in China, liver cancer has the fastest growing death rate. HCC is highly malignant with considerable metastatic potential, which underlines the necessity for developing novel potential drug candidate. Guttiferone K (GUTK), a bioactive polyprenylated acylphloroglucinol derivative isolated from Garcinia yunnanensis $\mathrm{Hu}$ in Bull. Fan., has been proved to suppress the proliferation of human colorectal cancer cells in our previous study. The present study is to determine whether or not GUTK could inhibit HCC metastasis.

Methods: Proteomic analysis was used 2D gel electrophoresis followed by Mass spectrometry. The expression of p115RhoGEF, p-Rac1, Rac1, Diap1, and profilin 1 were determined by western blotting. The presence of $\beta$-tubulin and profilin 1 was confirmed by immunofluorescence staining.

Results: GUTK concentration- and time-dependently reduced the migration and invasion of human hepatoma cell HepG2 without noted cytotoxicity. GUTK is able to block metastasis of HCC in mice. The pulmonary metastasis of HepG2 injected via tail veins was significantly reduced over 28 days in a GUTK dose-dependent manner. At high dose $(10$ $\mathrm{mg} / \mathrm{kg}$ ), GUTK was more effective than 5 -FU (25 mg/kg). No toxicity was noted by body weight and macroscopic analysis of vital organs. Proteomic analysis identified 30 proteins that were differentially expressed (18 overexpressed and 12 underexpressed) response to GUTK in HepG2 cells. Profilin 1 was increased by 7.4 fold in the presence of GUTK, confirmed by western blotting. Subsequent study showed that siRNAmediated profilin 1 knockdown reversed the suppressive effect of HepG2 cell migration and invasion induced by GUTK. In addition, GUTK changed cytoskeleton and increased the expression of profilin 1 by immunofluorescence. GUTK impaired Arp2/3-dependent actin polymerization and suppressed RhoGEF/Rac1/Diap1 involved signaling pathway.

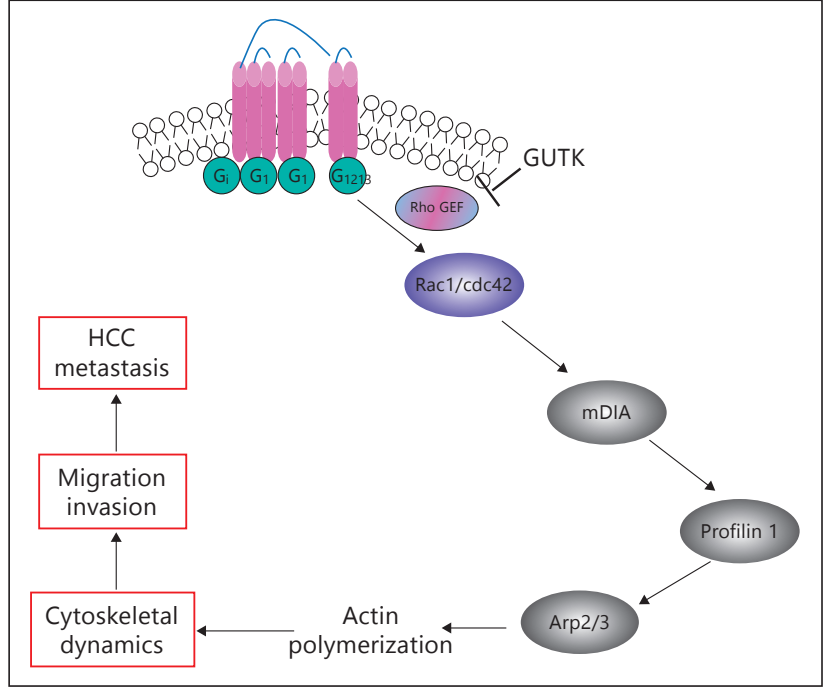

Fig. 1. Signaling pathway by which GUTK inhibited tumor metastasis in HCC (for abstract PS01-001).

Conclusion(s): The natural compound GUTK has the potential to prevent metastasis of HCC by restoration of profilin 1 expression (Figure 1). Also, present results imply that the silence of profilin 1 in HCC appears to be necessary and sufficient for metastasis of HCC.

\section{PS01-002 \\ Novel Retinoid, Peretinoin, Prevents HCC Recurrence Due to Multicentric Carcinogenesis after Radical Treatments; Survival Survey Result after Peretinoin Phase II/III Study \\ Kiwamu Okita $^{1 *}$, Hiromitsu Kumada², Masatoshi Kudo ${ }^{3}$ \\ ${ }^{1}$ Graduate School of Medicine, Yamaguchi University, Shimonoseki, Japan; ${ }^{2}$ Department of Hepatology, Toranomon Hospital Kajigaya, Kanagawa, Japan; ${ }^{3}$ Department of Gastroenterology and Hepatology, Kinki University School of Medicine, Osaka, Japan}

Introduction: Hepatocellular carcinoma (HCC) has a high recurrence rate and a poor prognosis even after curative treatment and the majority of reason for death is HCC recurrence. Acyclic retinoid, peretinoin, was suggested to have 


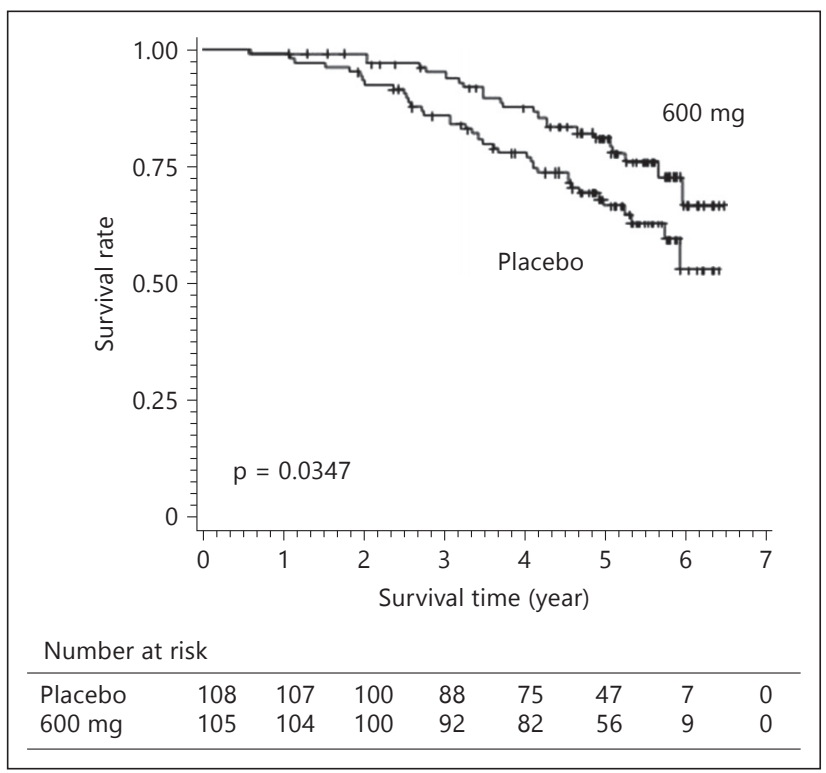

Fig. 1. Kaplan-Meier survival curves of Child-Pugh class A patients (for abstract PS01-002).

preventive effects on recurrence, particularly multicentric carcinogenesis, at the dose level of $600 \mathrm{mg} /$ day by a phase II/ III study with patients after HCC treatments. This survey aimed to evaluate the peretinoin's effects on survival after 3 years of phase II/III study completion.

Methods: The phase II/III study was a multicenter, double-blind, placebo-controlled, randomized study on HCV positive HCC patients. Of 401 registered patients were randomized to peretinoin $600 \mathrm{mg} /$ day, peretinoin $300 \mathrm{mg} /$ day, or placebo group. The maximum duration of study drug administration was 2 years. All patients who received the treatments, 392 patients from 41 facilities that performed the study, were analyzed for this survey; those patients received study drug only in phase II/III study period. The timing of this survey was designed to achieve median follow-up time of 5 years. Primary endpoint was overall survival. The main comparison was made between $600 \mathrm{mg}$ group and placebo group, and subgroup analysis on Child-Pugh A (CP-A) patients was performed based on the international recommendation. Comparison of the groups was performed by log-rank test, and hazard ratio was estimated by Cox regression analysis.

Results: The median follow-up time was 4.9 years. Analyzed patients were 132 in 600 mg group and 129 in placebo group. Number of deaths reported in $600 \mathrm{mg}$ group was 36 , and in placebo group was 47 and lost to follow-up were 19 and 15 in $600 \mathrm{mg}$ and placebo group, respectively. Survival at 2 years and 5 years in 600 mg group were $93 \%$ and $74 \%$ and those in placebo group were $93 \%$ and $64 \%$. There was no statistically significant difference on overall survival between $600 \mathrm{mg}$ and placebo group $(\mathrm{P}=0.1475$, HR: 0.726, 95\% CI: 0.470-1.122). For CP-A patients, 105 in 600 mg group and 108 patients in placebo group were analyzed. Survival at
2 years and 5 years were $99 \%$ and $81 \%$ in $600 \mathrm{mg}$ group and $94 \%$ and $67 \%$ in placebo group, respectively. Significantly longer survival was observed between in $600 \mathrm{mg}$ and placebo group ( $\mathrm{P}=0.0347$, HR: 0.575, 95\% CI: 0.341-0.967).

Conclusion(s): By $600 \mathrm{mg} /$ day of peretinoin, 5-year survival rate was higher than placebo group. Particularly for CP-A patients, $600 \mathrm{mg}$ group had significantly longer survival compared to placebo group. This survey strongly suggests that peretinoin $600 \mathrm{mg} /$ day improves overall survival by preventing HCC recurrence. Currently, study to verify the peretinoin's preventive effects on recurrence in CP-A patients after HCC treatments is ongoing.

Acknowledgements: We thank the patients and their families who took part in the study and all those involved in the research activities.

\section{PS01-009 \\ Incidence of HCC in Chronic Hepatitis B (CHB) Patients Receiving Entecavir and Lamivudine Therapy}

Yun Nah Lee, Hee Jun Kim Young Seok Kim*, Sang Gyune Kim, Jung Hwan Park, Sae Hwan Lee, Soung Won Jeong, Jae Young Jang, Hong Soo Kim, Boo Sung Kim

Digestive Disease Center and Research Institute, Department of Internal Medicine, Soon Chun Hyang University School of Medicine, Bucheon, Korea

Introduction: Although nucleos(t)ide analogues (NUCs) play a great role to reduce the incidence of HCC, it is not clear what factors contribute the occurrence of HCC during continuing treatment. The aim of this study was to assess and compare the incidence and risk factor for the development of HCC during long-term entecavir (ETV) and lamivudine (LAM) therapy in CHB patients.

Methods: Total $535 \mathrm{CHB}$ patients who have been treated with NUCs starting with ETV (293 patients) and LAM (242 patients) for more than 1 year were included. The incidence and the risk factors of HCC were analyzed.

Results: HCC developed in 19 of 293 (6.5\%) patients and 16 of $242(6.6 \%)$, respectively. The rate of LAM having undetectable HBV DNA at 1 year after treatment was lower when compared with the ETV-treated group (56.6\% vs $87.4 \%$, p < $0.001)$. The cumulative incidence rates of HCC was not significantly difference between ETV and LAM ( $p=0.175)$. In multivariable analysis, old age (OR 4.22; $\mathrm{p}=0.022$ ), failure to normalize ALT (OR 4.31; $\mathrm{p}=0.002$ ), LC (OR 3.31; $\mathrm{p}=0.009$ ) were independently associated with HCC development in ETV and LC (OR 5.87; $\mathrm{p}=0.005)$. In LAM, albumin lower than 3.8 $\mathrm{mg} / \mathrm{dL}$ (OR 3.68; $\mathrm{p}=0.028$ ) and diabetes mellitus (OR 3.51; $\mathrm{p}=0.015$ ) were independent risk factors of HCC development.

Conclusion(s): ETV therapy showed more excellent efficacy in nucleoside-naïve CHB patients than LAM therapy, 
but there was no difference in cumulative incidence rates of HCC. Therefore, the study about effect of antiviral potency in development of HCC will be needed.

\section{PS01-010 \\ External Validation of HCC Occurrence Risk Score Models in Patients with CHB in Korea \\ Kyu Sik Jung, Beom Kyung Kim*, Seung Up Kim, Jun Yong Park, Do Young Kim, Sang Hoon Ahn, Kwang-Hyub Han \\ Department of Internal Medicine, Yonsei University College of Medicine, Seoul, Korea}

Introduction: Recently, several risk scoring models for development of hepatocellular carcinoma (HCC) in chronic hepatitis B virus (HBV) infection were developed with promising results. However, prognostic performances of these systems still need to be validated more, especially in the era of antiviral therapy.

Methods: From 2005 to 2011, patients with chronic HBV infection were enrolled and followed-up for development of HCC. At enrollment, liver stiffness (LS) was measured in all patients using transient elastography (TE) and predictive performances of 5 HCC risk scores (CU-HCC score, CAG-HCC score, REACH-B score, LSM-HCC score, and modified REACH-B [mREACH-B] score) for HCC development were assessed with areas under the receiver operating characteristic curves (AUROCs). mREACH-B score was calculated using same mathematical formula of REACH-B score except serum HBV-DNA level being substituted with LS values, weighing 0,1 , and 2 points for an LS value $<8.0 \mathrm{kPa}, 8.0-13.0 \mathrm{kPa}$, and $>13.0 \mathrm{kPa}$, respectively.

Results: Among a total of 1308 subjects analyzed, the median age (883 men) was 50.0 years and all had wellpreserved liver function at enrollment. During follow-up (median 75.3 months), antiviral therapy was commenced in 848 patients (64.9\%) if necessary, and HCC developed in 125 (9.6\%) patients with a cumulative incidence of $1.78 \%$ per year. AUROCs of mREACH-B score at 3/5-year were 0.828 , 95\% CI [confidence interval] 0.779-0.876/0.806, 95\% CI $0.767-0.846)$ were higher than those of CU-HCC score $(0.698$ 95\% CI $0.628-0.767 / 0.700,95 \%$ CI $0.645-0.754)$, CAG-HCC score $(0.717,95 \%$ CI $0.688-0.814 / 0.757,95 \%$ CI $0.710-$ $0.804)$, REACH-B score $(0.717,95 \%$ CI $0.651-0.783 / 0.699$, 95\% CI 0.647-0.751), and LSM-HCC score $(0.777,95 \% \mathrm{CI}$, 0.722-0.831/0.759, 95\% CI 0.713-0.805).

Conclusion(s): Prognostic performances of mREACH-B score seemed better compared to other models including REACH-B model, suggesting the importance of fibrotic burden in the era of antiviral therapy rather than serum HBV-DNA level as a conventional biomarker.

\section{PS01-011 \\ Prevention of HCC in HCV Carriers with Food Supplement \\ David C.P. Chen $^{1 *}$, Christopher Duong Bui ${ }^{2}$ \\ ${ }^{1}$ Radiology, University of S. California, Los Angeles, United States; ${ }^{2}$ Medicine, Chris Bui Liver Disease Clinic, Orange County, United States}

Introduction: Hepatocellar carcinoma (HCC) is the most common cancer in the world. Hepatitis B or C(HCV) is the major cause of liver cancer. The total population of hepatitis $\mathrm{C}$ is 170 million. They represent $5 \%$ of total population. Generally, about 20 to 30 years later, $20-30 \%$ of HCV carriers will develop to cirrhosis and HCC. High virus load is the key to predict liver cancer. Reducing the virus load is an important issues in management the HCV carriers. Current therapy is interferon with Ribavirin and with about $30-50 \%$ cure rate. However, the side effects of interferon are severe such as general weakness, nausea, vomiting, low WBC and platelet and loss of hair. Therefore, searching for an alternative therapy is needed. We developed a combination of immune regulatory factors with herbs (Liver Strong), which can overcome the problems. This abstract reports our preliminary results.

Methods: Twenty-one hepatitis C carriers were included. They took these supplements two capsules, two times a day. General conditions, blood liver function and virus counts were performed at month 0 and month 3,6 and 9. The quantitative virus counts were performed with PCR method in the same lab. The results were evaluated with pair-Student's T test for statistics significance.

Results: In 21 hepatitis C carriers, 15 of them (71\%) were improved in general conditions, blood liver function and virus counts. For those improved cases, averagely about 90\% reducing rate of virus counts in hepatitis $\mathrm{C}$ carriers in month 3 . Five of 21 patient $s$ do not response to the treatment of Liver Strong. Three of them (60\%) are Type Ib. One is Type VI and the other is Type II.

Conclusion(s): Our animal study performed on mice showed increased natural killer cells killing activity and interferon-r. These two factors are important for suppressing and killing the virus within and outside the liver cell. With the success in animal study, we did a clinical trial of this supplement for hepatitis $\mathrm{C}$ carriers. The preliminary results are much encouraged with good reduction of virus counts without any side effect. The future plan will be a double blind study and isolated the key peptide within this compound. 


\section{PS01-013 \\ Characteristics of Patients with Hepatocellular Carcinoma in Taiwan: Analysis of National Cancer Registration Data in 2011}

Sheng-Nan Lu" ${ }^{1 *}$ Ching-Shui Huang ${ }^{2}$, Chen-Chun Wu ${ }^{3}$ Tsang-Wu Liu' ${ }^{4}$, Xiu-Ling Lin ${ }^{5}$, Chein-Fu Hung, Yee-Min Jen ${ }^{7}$, Shiu Feng Huang ${ }^{8}$, Jui-Ting $\mathrm{Hu}^{9}$, Chun-Ju Chiang ${ }^{10}$, Chien-Hung Chen ${ }^{11}$, Gar-Yang Chau ${ }^{12}$

${ }^{1}$ Division of Hepatogastroenterology, Department of Internal Medicine, Kaohsiung Chang Gung Memorial Hospital and Chang Gung University College of Medicine, Kaohsiung, Taiwan; ${ }^{2}$ Division of General Surgery, Cathay Medical Center, Kaohsiung, Taiwan; ${ }^{3}$ Department of Surgery, Taichung Veterans General Hospital, Taichung, Taiwan; ${ }^{4}$ National Institute of Cancer Research, National Health Research Institutes, Zhunan, Taiwan; ${ }^{5}$ National Taiwan University Hospital, National Taiwan University College of Medicine, Taipei, Taiwan; ${ }^{6}$ Department of Diagnostic Radiology, Linkou Chang Gung Memorial Hospital, Taoyuan, Taiwan; ${ }^{7}$ Departments of Radiation Oncology, Tri-Service General Hospital, Taipei, Taiwan; ${ }^{8}$ Division of Molecular and Genomic Medicine, National Health Research Institute, Taipei, Taiwan; ${ }^{9}$ Director of Liver Unit, Cathay Pacific Medical Center, Taipei, Taiwan; ${ }^{10}$ Taiwan Cancer Registry, Taiwan Cancer Registry, Taipei, Taiwan; ${ }^{11}$ Department of Internal Medicine, National Taiwan University Hospital and National Taiwan University College of Medicine, Taipei, Taiwan; ${ }^{12}$ Division of General Surgery, Department of Surgery, Taipei Veterans General Hospital, Taipei, Taiwan

Introduction: Although several published papers reported the characteristics of hepatocellular carcinoma (HCC) in Taiwan, it should be local and previous data. The National Cancer Registration included Barcelona Clinic of Liver Cancer (BCLC) staging system from 2007, and added site-specific factors (SSF) in 2011. It makes possible to have updated nationwide information.

Methods: From the National Cancer Registration database, we selected cases with HCC (ICD $9=155.0$ ), those who were diagnosed in 2011 and analyzed their sociodemographic information, SSF and BCLC staging.

Results: A total of 7942 cases were enrolled. Among them 7927 (99.8\%) met at least one cytological/pathological (51\%), radiological $(31 \%)$, serological alpha-fetoprotein (AFP) $>200 \mathrm{ng} / \mathrm{ml}(17.8 \%)$ diagnosis criteria of HCC. There were 5709 (71.9\%) men and 2233 (28.1\%) women. The male to female ratio was 2.56 . Their mean age was 63.6 years with a standard deviation of 12.6 years. Among them, $40.4 \%$ have a AFP level less than $20 \mathrm{ng} / \mathrm{ml}$ and another $23.9 \%$ have a AFP level between 20 and $200 \mathrm{ng} / \mathrm{ml}$. Limiting the 2116 cases underwent surgical treatment, 927 (43.8\%) showed patho- logic cirrhosis (Ishak fibrosis score, F5 and F6). Among 4961 cases without surgical treatment and available image information, $73.9 \%$ had cirrhosis. Concerning Child-Pugh classification, $61.6 \%$ were class $\mathrm{A}, 16.6 \%$ were class B, $5.9 \%$ were class $C$ and $15.8 \%$ had not available information. Twenty-one percent of male and $8.3 \%$ of female patients have higher creatinine levels. For HBsAg carrier status, $36.7 \%$ were with positive laboratory results, another $7 \%$ had positive history, $47 \%$ were negative for laboratory test and/or family history, and $8.8 \%$ had not available information. For anti-HCV, they were $29.6 \%, 7 \%, 55 \%$ and $8.7 \%$, respectively. There were $5.7 \%, 35.6 \%, 25 \%, 24.6 \%$ and $9.2 \%$ in BCLC stages $0, A, B, C$ and $\mathrm{D}$, respectively.

Conclusion(s): This report provided current status of incident HCC patients in Taiwan.

\section{PS01-015 \\ Risk Factors of Hepatocellular Carcinoma in Korean Patients with Antiviral Treatment for Chronic Hepatitis B: A Nationwide, Retrospective Cohort Study in Real-Life Setting}

T.Y. Kim ${ }^{1}$, J.H. Sohn ${ }^{1 *}$, W.Y. Tak ${ }^{2}$, B.H. Han ${ }^{3}$, W.J. Chung ${ }^{4}$, H.J. Min ${ }^{5}$, H.P. Shin', S.W. Nam ${ }^{7}$, I.H. Kim ${ }^{8}$

${ }^{1}$ Gastroenterology, Hanyang University Guri Hospital, Guri, Korea; ${ }^{2}$ Gastroenterology, Kyungpook National University Hospital, Daegu, Korea; ${ }^{3}$ Gastroenterology, Kosin University College of Medicine, Busan, Korea; ${ }^{4}$ Gastroenterology, Keimyung University Dongsan Medical Center, Daegu, Korea; ${ }^{5}$ Gastroenterology, Gyeongsang National University Hospital, Jinju, Korea; ${ }^{6}$ Gastroenterology, Kyung Hee University Hospital at Gandong, Seoul, Korea; ${ }^{7}$ Gastroenterology, The Catholic University of Korea, Daejeon St. Mary's Hospital, Daejeon, Korea; ${ }^{8}$ Gastroenterology, Chonbuk National University College of Medicine, Jeonju, Korea

Introduction: This study aimed to analyze risk factors of hepatocellular carcinoma (HCC) development in Korean patients with oral antiviral treatment for chronic hepatitis B (CHB) in real-life practice setting.

Methods: Clinical data were collected retrospectively but consecutively from 1,028 patients who were naïve and started oral antiviral treatment at nationwide-located 8 academic hospitals from January 2007 to December 2009. Among them, 164 were excluded because of pre-existing HCC $(n=136)$, HCC development within 6 months $(n=8)$, and initial drug-resistant mutation within 6 months $(\mathrm{n}=20)$. Finally 864 patients treated with entecavir $(n=408)$ or nucleoside analogues $(n=456)$ were analyzed. Cumulative incidence and independent risk factors for HCC development were analyzed by Kaplan-Meier curve and multivariable Cox proportional hazards model, respectively. 
The 5th Asia-Pacific Primary Liver Cancer Expert Meeting (APPLE 2014)

Results: During a median follow-up of 3.04 (range 0.5-5.0) years, HCC developed in 54 patients (6.3\%). Cumulative incidences of HCC were significantly different between non-cirrhotic and cirrhotic patients $(0 \%, 0.5 \%, 1.6 \%, 3.0 \%$ vs. $3.0 \%, 6.8 \%, 12.4 \%, 19.0 \%$ at 1-, 2-, 3-, and 4-year; $\mathrm{P}<0.001$ ). HCC risk was highest in patients with baseline HBV DNA level of 6-8 $\log 10$ copies $/ \mathrm{ml}$ than other levels $(\mathrm{P}=0.016)$, with cumulative incidences of $1.9 \%, 4.3 \%, 7.4 \%, 11.4 \%$ at $1-, 2-, 3-$, and 4-year. However, there was no significant difference according to the type of initial antiviral agents and 6-month complete virological response $(\mathrm{P}=0.231$ and 0.541 , respectively).

Independent risk factors for HCC on multivariate analysis were age per 10 years (HR 2.01, 95\% CI 1.34-3.02, P = 0.001), baseline serum AFP > 20 ng/ml (HR 4.47, 95\% CI 1.99-10.06, $\mathrm{P}<0.001$ ), presence of cirrhosis (HR 6.38, 95\% CI 1.89-21.53, $\mathrm{P}=0.003$ ) and HBV DNA level of 6-8 $\log 10$ copies $/ \mathrm{ml}$ (HR $5.10,95 \%$ CI 1.73-15.05, $\mathrm{P}=0.003)$. In subgroup of cirrhosis, still 3 other factors were significant.

Conclusion(s): In real life setting, age, presence of cirrhosis, baseline AFP and HBV DNA levels are significant risk factors for HCC in CHB patients even after oral antiviral treatment, whereas the type of initial antiviral agents and 6 month complete virological response are not significant.

\section{PS01-016 \\ A Comparative Study on Hepatitis C and Hepatitis B Infection on Viral Hepatitis- Associated Hepatocellular Carcinoma - Cohort from Liver Center}

Alvina Ahmed, Muhammad Memon*

Gastroenterology \& Hepatology, Asian Institute of Medical Sciences, Hyderabad, Pakistan

Introduction: Globally, Hepatocellular carcinoma (HCC) took the third place in cancer related mortality and is also thought to be the first cause of death in cirrhotic patients. In Pakistan prevalence of HCC varies from $3.7 \%$ of all malignant tumors to $16 \%$. A great proportion of HCC is caused by viral infections mainly hepatitis $\mathrm{C}$ virus (HCV) and hepatitis $\mathrm{B}$ virus (HBV). Available data focused on the HCV-HCC group or HBV-HCC group, but to our best knowledge till now no study was conducted from South-east Asia that shows combined prevalence of HCV-HCC and HBV-HCC along with combined clinical and pathological association of these viruses with HCC. Therefore, this study was indicated for estimating the true prevalence of HCV and HBV related HCC and also we explored the demographic and clinical-pathological association among them.

Methods: This cross sectional observational study enrolled clinical data base of HCC patients who attended the Asian Institute of Medical Sciences (AIMS) hospital, Hyderabad, Pakistan between 2011 to 2013. HCC was diagnosed on the characteristic features on CT scan/MRI. Variables included from baseline; age, sex, area of residence to Clinical-pathological features such as underlying co-morbidity, presence or absence of liver cirrhosis, Macrovascular involvement of HCC, tumor extension and metastasis, liver lobes involved, serum alpha-Fetoprotein level, and hepatitis serologies in 188 patients.

Results: Overall prevalence of HCV and HBV related HCC was $65.95 \%$ and $30.24 \%$, respectively. Patients with HCV were more likely to develop HCC at advanced age $(52.35 \pm 11.9$ vs. $40.67 \pm 12.09$ - years), predominantly male $62 \%$ (HBV $75.4 \%$ ), highly raised serum AFP levels ( $\geq 400 \mathrm{ng} / \mathrm{ml}$ ) $78.2 \%$ (HBV 67.1\%), large tumor size (HCV-66\% >5 cm, HBV-59.3\% $>5 \mathrm{~cm}$ ), and presence of portal vein thrombosis $8.06 \%$ (HBV $1.56 \%$ ). A binominal multivariate analysis showed that HCC due to HCV infection were more likely to be cirrhotic $(\mathrm{OR}=$ $0.245,95 \%$ CI: $0.117,0.516$ ) and had more than two times higher rate of solitary Macrovascular involvement $(\mathrm{OR}=$ 2.533, 95\% CI: 1.162, 5.521) as compared with HBV associated HCC; $\mathrm{p}<0.05$.

Conclusion(s): Statistically significant variations were observed from baseline to clinical-pathological characteristics in HCV vs. HBV associated HCC. Our study suggests prompt and early screening for HCC in high risk patients so that the rate of progression of these chronic viral diseases to cirrhosis and cancer can be decreased. 


\section{Category 2 \\ Prediction of HCC Recurrence}

\author{
PS02-003 \\ Is Hepatitis B SuRFAce Antigen (HBsAg) \\ Quantification Associated with Aggressive \\ and/or Recurrent Hepatocellular Carcinoma \\ (HCC)? \\ Adrian Zhongxian Poh ${ }^{1 *}$, Yi Hao Clement Lin ${ }^{1}$, \\ Kwai Peng Chan' ${ }^{2}$ Wan Cheng Chow', \\ Pik-Eu Jason Chang ${ }^{1}$, Boon Bee George Goh ${ }^{1}$, \\ Hiang Keat Tan ${ }^{1}$, Chee Kiat Tan ${ }^{1}$ \\ ${ }^{1}$ Gastroenterology \& Hepatology, Singapore General \\ Hospital, Singapore; ${ }^{2}$ Pathology, Singapore General \\ Hospital, Singapore
}

Introduction: High transcriptional activity of cccDNA within hepatocytes indicates less sustained viral suppression. Serum HBsAg titre, which correlates with hepatocyte cccDNA transcriptional activity, is useful in predicting HCC in patients with HBV DNA $<2000 \mathrm{IU} / \mathrm{ml}$. There is no data on its association with aggressive and/or recurrent HCC. We aim to study the association of HBsAg quantification and other parameters with aggressive and/or recurrent HCC in CHB patients.

Methods: In this cross-sectional case control study, serum samples from 172 HCC patients collected between 2004 to 2010 were screened. Inclusion criteria were CHB infection and HCC. Eighty-five were excluded due to HBsAg negativity, HCV co-infection, inadequate serum for analysis and insufficient clinical data. Another 31 were excluded due to loss to follow-up (3), non-liver related death (2), declined treatment (3) and serum collection exceeding 6 months from date of diagnosis/treatment (23). The study group comprised of cases with aggressive (microvascular invasion, portal vein involvement or metastases), multifocal and/or recurrent HCC. Recurrence is defined as HCC recurring within 2 years of initial HCC treatment. Controls were those with unifocal HCC without recurrence. HBsAg was quantified using Architect HBsAg assay, a chemiluminescent microparticle immunoassay from Abbott Laboratories, in accordance with the manufacturer's instructions.

Results: There were 40 cases and 16 controls. Treatment in the study group included resection (11), TACE (4), RFA (2), PEIT (2), capecitabine (2), SIR-Spheres (1) and thalidomide (1). The remaining 17 did not receive any treatment. In the control group, 15 underwent resection and 1 received RFA. Baseline characteristics between study and control groups were similar in terms of age, serum ALT, AST, HBeAg status, presence of cirrhosis and the use of anti-viral agents. Mean HBsAg quantification titre was higher in the study group (1739.1 $\pm 4536.12 \mathrm{IU} / \mathrm{mL}$ vs $1363.4 \pm 978.12 \mathrm{IU} / \mathrm{mL} ; \mathrm{p}=0.132$ ). There were more smokers $(62.2 \%$ vs $22.2 \% ; \mathrm{p}=0.059)$ and presence of diabetes mellitus ( $52.6 \%$ vs $20.0 \%$; $=0.084$ ) in the study group. The following were significantly higher in the study group: male gender ( $95.0 \%$ vs $62.5 \% ; p=0.005)$, serum ALP (154 U/L vs $30 \mathrm{U} / \mathrm{L} ; \mathrm{p}<0.0001)$, AFP (477.0 umol/L vs $10.1 \mathrm{umol} / \mathrm{L} ; \mathrm{p}=0.006)$ and median diameter of HCC $(7 \mathrm{~cm}$ vs $4 \mathrm{~cm} ; \mathrm{p}=0.035$ ). Multivariable analysis was not performed due to small sample size.

A sub-group of 35 with only single HCC lesion who underwent resection/RFA were further analysed. Eight out of the 35 recurred within 2 years and were labelled as the study group. The remaining 27 were the control group. Baseline characteristics between study and control groups were similar in terms of age, gender, serum ALT, AST, ALP, HBeAg status, presence of cirrhosis and anti-viral agents. HBsAg titres were lower in study group $(981.7 \pm 925.9 \mathrm{IU} / \mathrm{mL}$ vs $1362.6 \pm 1868.9 \mathrm{IU} / \mathrm{mL} ; \mathrm{p}=0.132$ ). However, median diameter of HCC ( $5 \mathrm{~cm}$ vs $3 \mathrm{~cm} ; \mathrm{p}=0.026$ ) was significantly higher in the study group.

Conclusion(s): HBsAg titres were not shown to be associated with aggressive and/or recurrent HCC in this study. Factors significantly associated with aggressive and/or recurrent HCC include male gender, serum ALP, AFP and diameter of HCC. The limitations of our study were its small sample size and being a retrospective study. Larger prospective studies are needed to confirm these associations.

\section{PS02-005 \\ The Prediction for Tumor Progression and Survival after Transarterial Chemoembolization in Intermediate Stage Hepatocellular Carcinoma Using 18F-Fluorodeoxyglucose Positron Emission Tomography \\ Min Jin Kim 1 , Jung Hwan Park' ${ }^{1}$ Young Seok Kim ${ }^{1 *}$, Sang Gyune Kim¹, Sae Hwan Lee ${ }^{1}$, Hee Jun Kim¹, Soung Won Jeong', Jae Young Jang', Hong Soo Kim', Won Hyung Lee'2, Jung Mi Park' ${ }^{2}$ Jae Myung Lee ${ }^{3}$, Min Hee Lee ${ }^{3}$, Deuk Lin Choi ${ }^{3}$ \\ ${ }^{1}$ Digestive Disease Center and Research Institute, Department of Internal Medicine, Soon Chun Hyang University School of Medicine, Bucheon, Korea; ${ }^{2}$ Nuclear Medicine, Soon Chun Hyang University School of Medicine, Bucheon, Korea; ${ }^{3}$ Radiology, Soon Chun Hyang University School of Medicine, Bucheon, Korea}

Introduction: 18F-Fluorodeoxyglucose (FDG) positron emission tomography (PET) has been used to assess the biological behavior of hepatocellular carcinoma (HCC). In this 
study, we investigated the usefulness of 18F-FDG PET usefulness for evaluating tumor progression and survival of intermediate Barcelona Clinic Liver Cancer (BCLC)-stage HCC patients treated with transarterial chemoembolization (TACE).

Methods: From February 2006 to March 2013, 210 patients treated with TACE, including BCLC intermediatestage HCC patients, were examined via 18F-FDG PET. FDG uptake was calculated based on tumor maximum (Tmax) standardized uptake values (SUVs), liver mean (Lmean) SUVs, and the ratio of maximal tumor SUV to mean liver SUV (Tmax/ Lmean).

Results: The mean follow-up period for the 77 patients (52 males, 25 females, average age 63.3 years) was 22.2 months. The mean number of TACE sessions was 2.4. Average tumor size and number were $5.6 \mathrm{~cm}$ and 3.3, respectively. The mean Tmax and Tmax/Lmean values were 2.1 \pm 3.0 and $2.4 \pm 1.3$, respectively. Multivariate analysis revealed that Tmax/Lmean was an independent predictor of overall survival (hazard ratio [HR] 1.96, 95\% confidence interval [CI] 1.2103.156, $\mathrm{P}=0.006)$. In addition, Tmax/Lmean was an independent predictor of tumor progression (HR 2.05, 95\% CI 1.264-3.308, $\mathrm{P}=0.004)$.

Conclusion(s): FDG uptake calculated with Tmax/Lmean using PET predicted the tumor progression and survival of BCLC intermediate-stage HCC patients who underwent TACE.

\section{PS02-010 \\ Inter Alpha Inhibitor H4 and Carbonic Anhydrase I as Potential Prognostic Factors in Patients with Hepatocellular Carcinoma Treated with Radiotherapy}

\author{
Hyejung Cha Eun Jung Lee, Seung Hyun Yang, \\ Geun Yeong Jeong, Jinsil Seong* \\ Radiation oncology, Yonsei University College of \\ Medicine, Seoul, Korea
}

Introduction: The early prognosis of treatment outcome is essential to increase treatment efficacy and survival in patients with hepatocellular carcinoma (HCC) treated with radiotherapy (RT). In this study, we carried out two-dimensional electrophoresis (2D) followed by proteins profiling to identify the prognostic factors in HCC patients treated with RT.

Methods: Each ten patients with good and poor treatment outcome were selected. Patients with poor treatment outcome were defined as patients showing short progression-free survival. Blood samples were collected from each patient before and after RT. 2D analysis was conducted in pooling serum of each group and the protein profiling was carried out by matrix-assisted laser desorption/ionization time-offlight mass spectrometry (MALDI-TOF MS) to compare the serum proteomic profiles of two groups. Candidate peaks were processed to partial purification, followed by protein identification by amino acid sequence analysis. Identified proteins were confirmed by the enzyme-linked immunosorbent assay (ELISA).

Results: Patient, tumor, and treatment characteristics between two groups were not different significantly. Three proteins were significantly increased ( $>5$ fold) in post-RT serum of group with good treatment outcome compared to pre-RT and two were significantly decreased ( $<5$ fold) in post-RT serum of group with poor treatment outcome compared to pre-RT. WE identified four proteins that were up-regulated by RT, including inter alpha inhibitor $\mathrm{H} 4$, fibrinogen gamma chain and keratin 9/1 complex in group of good treatment outcome. Three proteins decreased by RT that were identified, including carbonic anhydrase I and carbonmonoxy hemoglobin S in group of poor treatment outcome. These were confirmed by ELISA in each patient's sera as results inter alpha inhibitor $\mathrm{H} 4$ and carbonic anhydrase I were confirmed as identical results.

Conclusion(s): In this study, serum proteins profiling of patient with HCC treated with RT revealed that Inter alpha inhibitor $\mathrm{H} 4$ and Carbonic anhydrase I could be potential prognostic factors.

\section{PS02-011 \\ Impact of HBV DNA Level \& Antiviral Agent on the Recurrence of Patients after Liver Resection for HBV Related Hepatocellular Carcinoma \\ Tae Yoo ${ }^{1}$, Kyung-Suk Suh ${ }^{2 *}$, Kwang-Woong Lee ${ }^{2}$, Nam-Joon Yiं ${ }^{2}$, Hae Won Lee ${ }^{2}$, Geun Hong ${ }^{2}$, YoungRok Choi ${ }^{2}$, Hyeyoung Kim², Min-Soo Park ${ }^{2}$, Sukwon Suh' ${ }^{2}$, Jung Moo Lee ${ }^{2}$ \\ ${ }^{1}$ Surgery, Dongtan Sacred Heart Hospital, Hallym University College of Medicine, Seoul, Korea; ${ }^{2}$ Surgery, Seoul National University College of Medicine, Seoul, Korea}

Introduction: To investigate the significance of HBV DNA levels \& antiviral agent for predicting recurrence in HCC patients who underwent curative liver resection.

Methods: From 2005 to 2010, 341 HBV related HCC patients who underwent tumor resection in Seoul National University Hospital were enrolled. HBV DNA levels (pre-, postop. viral load) and antiviral treatment were analyzed for association with HCC recurrence, together with other clinical variables.

Results: Of the 294 patients, patients $(n=197)$ with low preop. HBV DNA level (2.5 X 100000 IU) had better recurrence outcome than those with high level $(p=0.01)$. Also in postop. period, the group $(n=164)$ with low viral titer(1 X $1000 \mathrm{IU}$ ) had better outcome than those with high viral load in recurrence $(\mathrm{p}<0.001)$. In terms of Antiviral agents, 


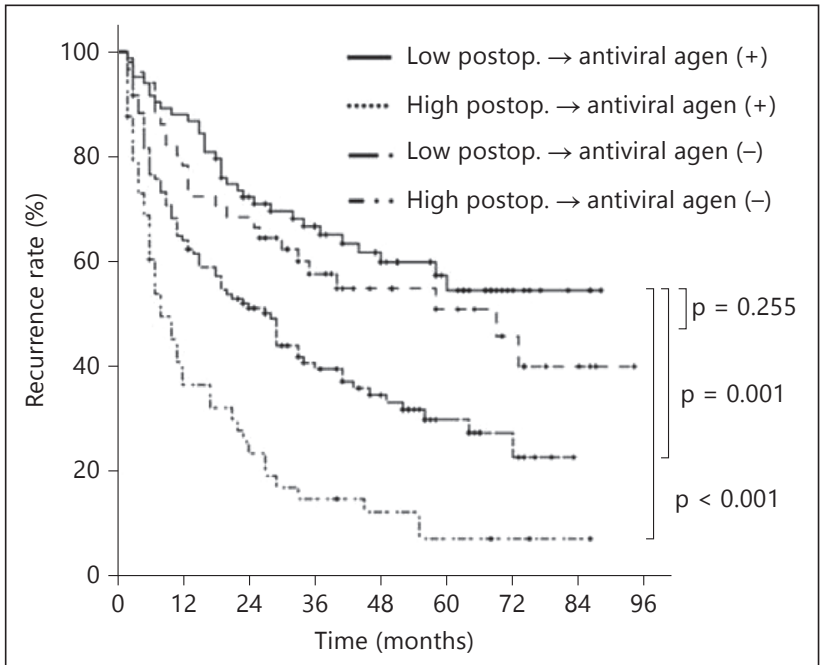

Fig. 1. Figure shows the recurrence outcome of HCC patients who underwent liver resection. If the antiviral treatment group had high HBV viremia postoperatively, they had good recurrence free survival (RFS) as group with low viremia (5yr RFS $=54.1$ vs. $58.3 \%$ ). But, even if untreated patients had low viremia, they had poor outcomes as untreatment group with high viremia (5-yr RFS $=37.1$ vs. $11.9 \%$ ) (for abstract PS02-011).

untreatment group $(n=136)$ had worse outcome than treatment group in recurrence $(p<0.001)$. In subgroup analysis, if the treatment group had high HBV viremia postoperatively, they had good RFS as group with low viremia. But, even if untreated patients had low viremia, they had poor outcomes as untreatment group with high viremia ( $\mathrm{p}<$ 0.001). Moreover, whether it is advanced stage (III) or not, low postop. HBV viral load showed the better recurrence outcome but, antiviral treatment did not present difference in advanced stage. Finally, postop AFP levels (HR = 3.52, $\mathrm{p}<$ 0.001), postop HBV DNA (HR $=2.2, \mathrm{p}<0.001)$, antiviral therapy ( $\mathrm{HR}=2.28, \mathrm{p}<0.001)$, tumor size $(\mathrm{HR}=1.54, \mathrm{p}=$ $0.025)$ \& microvascular invasion ( $H R=1.997, p=0.005)$ were independent risk factors for RFS in multivariated analysis.

Conclusion(s): Low HBV DNA load and antiviral therapy may be important factors after the curative treatment of HBVrelated hepatocellular carcinoma in terms of tumour recurrence. Therefore, to maintain the low level of HBV viremia, antiviral therapy should be considered after curative treatment of hepatocellular carcinoma.

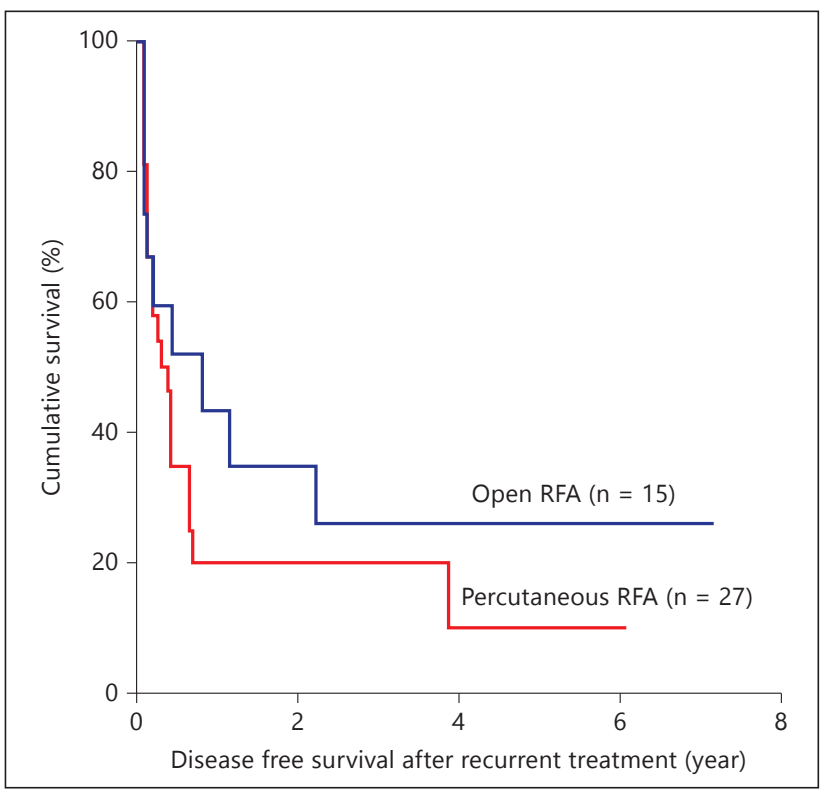

Fig. 1. Disease free survival after RFA for HCC recurrence (for abstract PS02-013).

\section{PS02-013 \\ Is Open RFA Better Than Percutaneous RFA for Recurrent Hepatocellular Carcinoma? A Ten-Year Review of a Single Center Practice

\author{
Peng Soon Koh*, Albert Chi Yan, Chi Yan Chan, \\ Tan To Cheung, Kenneth Siu-Ho Chok, \\ Wing Chiu Dai, Ronnie Tung-Ping Poon, \\ Chung Mau Lo
}

Department of Surgery, The University of Hong Kong, Hong Kong

Introduction: Recurrent hepatocellular carcinoma (HCC) following primary liver resection for HCC is common nowadays. The use of radiofrequency ablation (RFA) in the treatment of recurrent small HCCs had been shown to improve outcome and remained an alternative option to salvage transplantation or re-resection. We aim to compare the open surgical against the percutaneous RFA approach to HCC recurrence. The open surgical approach also offers the feasibility of RFA in difficult places to reach, which could not be performed by the percutaneous approach.

Methods: From January 2002 to December 2011, 495 patients developed recurrent HCCs following primary liver resection. 42 (8.5\%) of these patients underwent RFA as the initial treatment following recurrence. The overall survival and disease free survival from the period after RFA were performed in the percutaneous RFA group, $n=27$ (64.3\%) and open surgical RFA group, $\mathrm{n}=15$ (35.7\%) were compared.

Results: Patient demographics and the primary tumor characteristics of both groups were similar ( $p>0.05)$ except for 
the AFP levels ( $p=0.03$ ). In the percutaneous and open RFA group, the median survival were 27.5 and 29.0 months with 1-, 3 - and 5-year overall survival of $72.1 \%, 40.7$ and $20.4 \%$ versus $86.2 \%, 39.2 \%$ and $31.3 \%$ respectively $(\mathrm{p}=0.50)$. Mediandisease free survival was better in the open group than the percutaneous group (9.76 vs 3.65 months) although was not significant ( $p=0.32$ ) with a 1-, 3- and 5-year free survival of 43, 2\%, 25.9\% and $25.9 \%$ versus $19.8 \%, 19.8 \%$ and $9.9 \%$ respectively. A univariate and multivariate Cox regression analysis showed that high AFP levels $>500$ to be significant predictive factor for shorter overall survival (OR $=2.66,95 \%$ CI 1.19-5.96).

Conclusion(s): Open RFA offers the feasibility and added advantage for difficult tumor locations that could not be reached by the percutaneous approach. Although there is no significant difference in overall survival, a longer disease-free survival tends to favor with the open approach.

\section{PS02-014 \\ The Comparison of Current Staging Systems of Hepatocellular Carcinoma for Prognostic Stratification}

\author{
Jihye Je Ji Hoon Kim*, Yang Jae Yoo, Keunhee Kang, \\ Eileen L. Yoon, Hyun Jung Lee, Sang Jun Suh, \\ Seong Hee Kang, Hae Rim Kim, Young Kul Jung, \\ Yeon Seok Seo, Hyung Joon Yim, Jong Eun Yeon, \\ Soon Ho Um, Kwan Soo Byun \\ Internal Medicine, Korea University, Seoul, Korea
}

Introduction: Several prognostic stating systems have been proposed for hepatocellular carcinoma (HCC) and there has been debate regarding which prognostic staging system is the most accurate. We evaluated independent predictors of overall survival and compared 8 prognostic staging systems in patients with HCC.

Methods: A total of 533 patients with treatment-naive HCC who diagnosed and treated between 2007 and 2009 were included. Independent predictors of survival were assessed using the Kaplan-Meir test and the Cox proportional hazard model. And prognostic ability of each of the staging systems was expressed using time-dependent receiver-operating characteristics (ROC) curves.

Results: Median survival was 21.0 months, 54.8\% patients had chronic hepatitis B, and $58.5 \%$ patients had symptoms on present. In tumor characteristics, $59.3 \%$ had only one tumor nodule, $24.6 \%$ patients had major branch of portal vein thrombosis, $9.6 \%$ patients had distant metastasis. At the time of censorship, 361 (67.7\%) patients had died. The independent predictors of survival were ECOG performance status ( $p=0.005)$, Child-Pugh classification ( $p=0.014 / 0.008)$, serum AST levels $(p<0.001)$, serum AFP levels $(p=0.002)$, infiltrative tumor $(\mathrm{p}=0.004)$, the presence of portal vein thrombosis ( $p=0.002)$, hepatic vein thrombosis $(p<0.001)$, distant metastasis $(\mathrm{p}<0.001)$, and BCLC staging system ( $\mathrm{p}=$
$0.295 /<0.001 / 0.001 / 0.874)$. The cancer of the Italian program (CLIP) (area $=0.85$ ) and the Chinese University Prognostic Index (CUPI) (area $=0.87$ ) scoring systems had the best independent predictive power for survival compared with the other prognostic staging systems.

Conclusion(s): ECOG performance status, Child-Pugh classification, serum AST levels, serum AFP levels, infiltrative tumor, the presence of portal vein thrombosis, hepatic vein thrombosis, distant metastasis, and BCLC staging system were independent predictors of survival in patients with HCC. The CUPI, CLIP, JIS, UICC7, UICC6, and UICC5 (area $=0.87,0.85$, $0.83,0.81,0.80$, and 0.80 , respectively) staging system were comparable to stratify the prognosis of HCC patients, but CUPI was the best prognostic model in Korean cohort that hepatitis $B$ virus associated HCC were major population.

\section{PS02-016 \\ Associated Intervention Factors of Prognosis in Patients with Hepatocellular Carcinoma Underwent Non-Surgical Treatment}

\author{
Kwong-Ming Kee, Sheng-Nan Lu*, Jing-Houng Wang, \\ Chao-Hung Hung, Chien-Hung Chen \\ Division of Hepatogastroenterology, Department of \\ Internal Medicine, Kaohsiung Chang Gung Memorial \\ Hospital and Chang Gung University College of \\ Medicine, Kaohsiung, Taiwan
}

Introduction: Most of HCC staging systems predict prognosis of patients. However, most of determined factors, such as tumor involvement, AFP levels and liver function reserve, were unchangeable. In this study, we try to explore interventional factors of the prognosis in HCC cases underwent nonsurgical treatment.

Methods: All cases in a medical center in southern Taiwan from 2002 to 2012, and met all below criteria were enrolled in this study. They were (1) met consensus diagnostic criteria, (2) underwent the 1st two treatments in the Hospital (3) treated by non-surgical treatment and (4) viable cases should be observed form more than 5 year. Among them, 1136 (61.9\%) died within two years, 451 (24.6\%) died between 2 to 5 years after diagnosis (defined as Group A), and 247 (13.5\%) survived for 5 years or longer (defined as Group B). To compare interventional factors between groups A and $B$, the unchanged factors were matched using propensity score matching (PSM).

Results: Before matching, Group B had significantly better liver function reserve, earlier BCLC stage and low AFP levels. Furthermore, interventional factors, such as initial treatment by RFA, recurrent after 1 year, complete treatment of recurrence tumor, retreatment by RFA and anti-viral treatment, were also associated with good prognosis. After PSM ( $\mathrm{n}=247$ in each group), only BCLC staging, recurrent after 1 year, complete treatment of recurrence tumor and 
anti-viral treatment, were the only 4 significant factors. Limited in patients with BCLC stage 0 ( $\mathrm{n}=40$ in each group), complete treatment of recurrence tumor, and anti-viral treatment, were the only 2 significant factors $(p=0.036 \& p=$ $0.002)$. Limited in patients with BCLC stage A $(n=123$ in each group), recurrent after 1 year and complete treatment of recurrence tumor were the only two significant factors ( $p=$ $0.030 \& p<0.001)$. Limited in patients with BCLC stage B ( $n=$ 46 in each group), anti-viral treatment was the only borderline significant factor $(\mathrm{p}=0.058)$. Since only 11 cases with BCLC stage $C$ in group B, no further analysis was conducted.

Conclusion(s): Recurrent after 1 year, complete treatment of recurrence tumor, and anti-viral treatment, were the only 3 significant interventional factors associated with good prognosis. Based on this study, we learn that we should try our best to obtain complete response in the loco-regional treatment, to detect recurrent tumor earlier and to provide anti-viral treatment if feasible.

\section{PS02-019 \\ The Pre-Treatment VEGF Level Is a Predictive Factor for Recurrence in Post Radiofrequency Ablated HCC Patients}

\author{
Wen-Juei Jeng, Wei-Ting Chen, Chen-Chun Lin, \\ Wei Teng, Chien-Hao Huang, Chun-Yen Lin, \\ Shi-Ming Lin* \\ Division of Hepatology, Chang-Gung Memorial \\ Hospital, Linkou, Taipei, Taiwan
}

Introduction: Hepatocellular carcinoma is one of the most common malignancies in the world. Although advance of image improve the detection rate of early HCC, long-term survival is still unsatisfactory for high recurrence rate, even after curative therapy. The pathogenesis of HCC is correlated with angiogenesis, which vascular endothelial growth factor (VEGF) plays a role in development, progression and prognosis. The aim of the study is to investigate the prognostic role of serum VEGF in the curable HCC under complete radiofrequency ablation.

Methods: We prospectively recruited 15 fresh diagnosed HCC patients with treatment naïve. All patients were treated with radiofrequency ablation (RFA) alone. The pre-treatment serum VEGF level was analyzed by flow cytometry method and correlate with clinical outcome. The pre-treatment clinical factors including age, gender, etiology, Child-Pugh score, BCLC stage, cytology grading, tumor size, are also evaluated.

Results: Among the 15 HCC patients, 6 patients encountered tumor recurrence while three of them had metastasis. 8 of the 15 patients are men. The median age is 70.9 (51-83), $46.7 \%$ of the 15 patients are chronic hepatitis B infection. $80 \%$ of the 15 patients are liver cirrhosis Child-Pugh grade A while others are grade B. Older age (77 v.s. 67 year-old, $p=0.026$ ), higher AFP level (9442 v.s. 38.5, $p=0.003$ ) are factors related to recurrence. The pre-treatment serum VEGF level was significantly higher in the recurrence group compared to the non-recurrence group (58.36 v.s. $11.4, \mathrm{p}=0.012$ ).

Conclusion(s): The pre-treatment serum VEGF level, older age, higher AFP level are predictive of recurrence in HCC patients received RFA.

\section{PS02-020 \\ Prognostic Value of Magnetic Resonance Imaging versus Computed Tomography for Hepatocellular Carcinoma Treated with Chemoembolization \\ Beom Kyung Kim ${ }^{1}$, Myeong-Jin Kim², Kyung Ah Kim', Chansik An ${ }^{2}$, Kyu Sik Jung ${ }^{1}$, Wonseok Kang ${ }^{1}$, Jun Yong Park ${ }^{1}$, Do Young Kim', Sang Hoon Ahn', Kwang-Hyub Han ${ }^{1}$, Seung Up Kim ${ }^{1 *}$ \\ ${ }^{1}$ Internal Medicine, Yonsei University College of Medicine, Seoul, Korea; ${ }^{2}$ Radiology, Yonsei University College of Medicine, Seoul, Korea; ${ }^{3}$ Radiology, The Catholic University of Korea, Seoul, Korea}

Introduction: Computed tomography (CT) and magnetic resonance imaging (MRI) play important roles in diagnosis and staging of hepatocellular carcinoma (HCC). However, prognostic roles of radiological characteristics are not yet determined.

Methods: Eighty-eight patients treated with chemoembolization were analyzed. Radiological parameters at baseline were assessed in all patients using both dynamic CT and MRI. Treatment responses were assessed using modified RECIST 4 weeks after the first chemoembolization.

Results: Gross vascular invasion (GVI), bile duct invasion, irregular tumor margin (ITM), peripheral ragged enhancement (PRE), and satellite nodules on CT or MRI were associated with non-response (stable disease or progression) after chemoembolization, respectively (all $p \leq 0.05$ ). GVI, ITM, and PRE on CT or MRI were also independently associated with poor overall survival (OS), respectively (all $\mathrm{p} \leq 0.05$ ). Based on these, prognostic scoring systems for CT and MRI were developed; 0, absence of all 3 features (GVI, ITM, and PRE); 1, presence of one feature; 2 , presence of two features; and 3 , presence of three features. After adjusting tumor size, tumor number, and alpha-fetoprotein level, both CT and MRI scores were independently associated with OS (both $\mathrm{p}<0.001$ ). Patients with CT or MRI score $\geq 2$ had a worse OS than those with score $<2$ (adjusted hazard ratios, 3.837 and 2.938, respectively). MRI-specific parameters such as signal intensity on T2- or T1-weighted images, fat signal, or hyperintensity on diffusion-weighted images did not have prognostic value (all $\mathrm{p}>0.05)$.

Conclusion(s): Radiologic parameters by CT and MRI are useful in biological characterization of tumors and prognostification for HCC treated with chemoembolization. 


\section{CT findings}
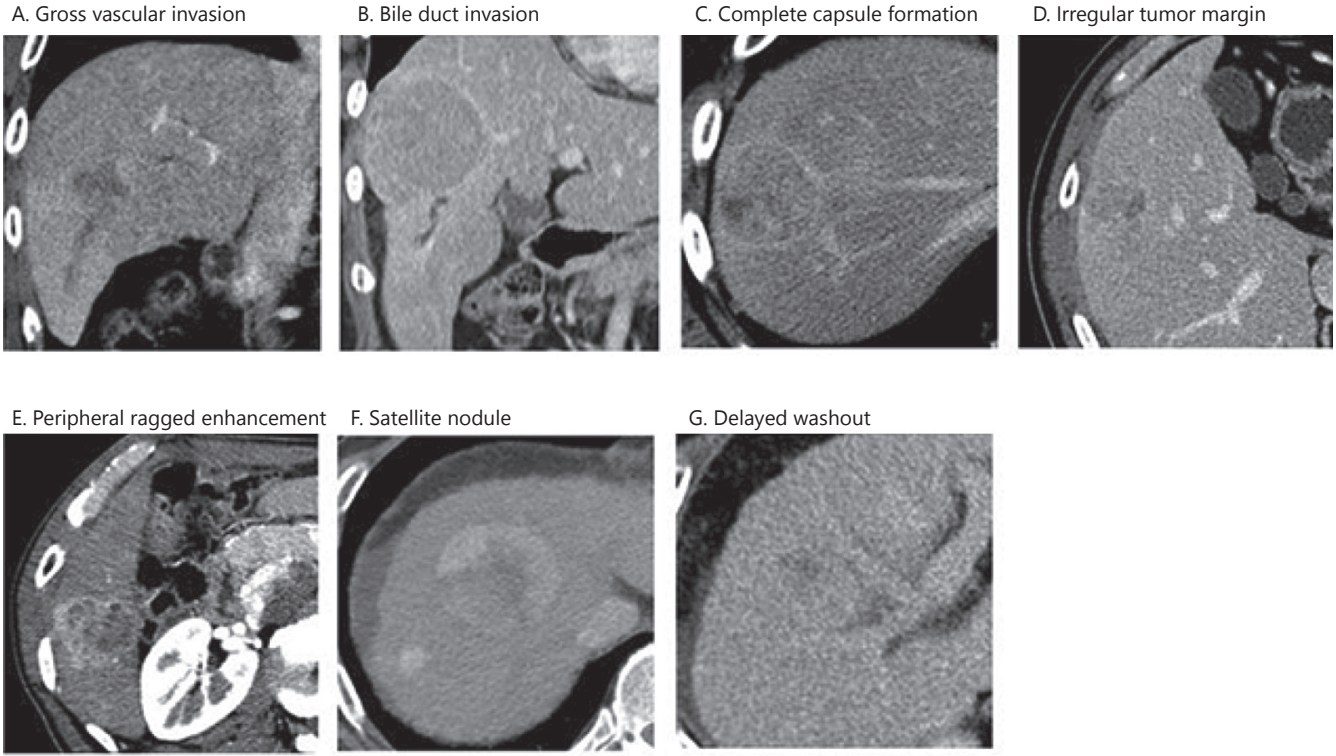

G. Delayed washout

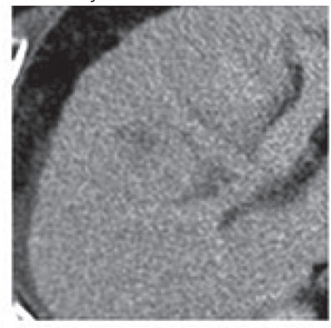

\section{MR findings}

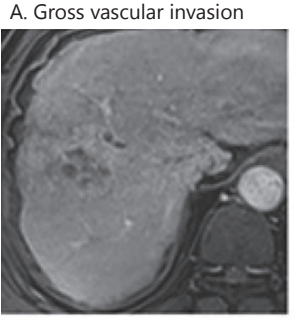

E. Peripheral ragged enhancement

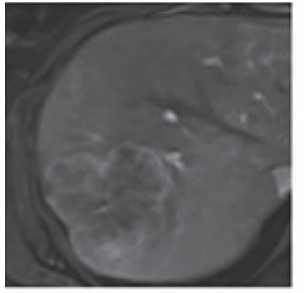

I. T1 hyperintense signal

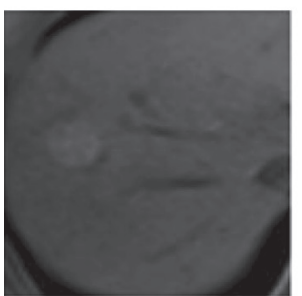

B. Bile duct invasion

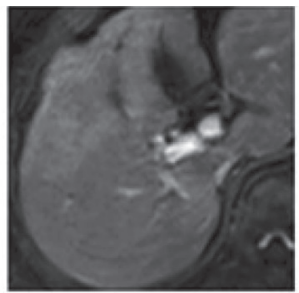

F. Satellite nodule

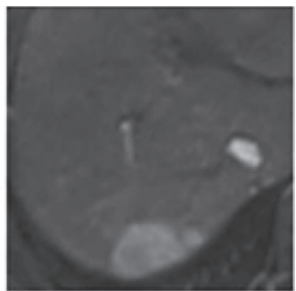

J. Fat component (in/out phase)

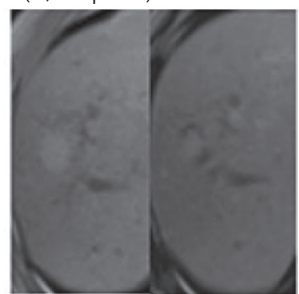

C. Complete capsule formation

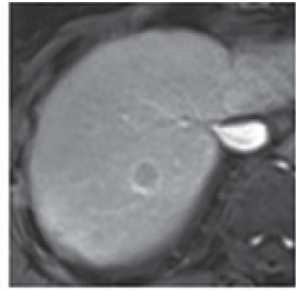

G. Delayed washout (arterial delayed phase)

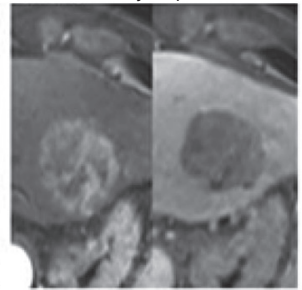

K. Hyperintense signal on DWI

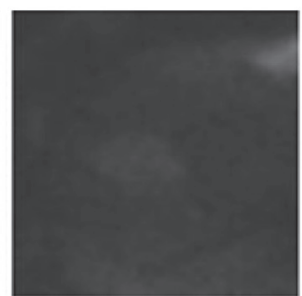

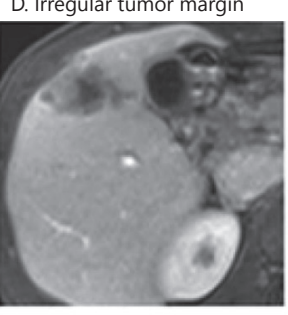

H. T2 hyperintense signal

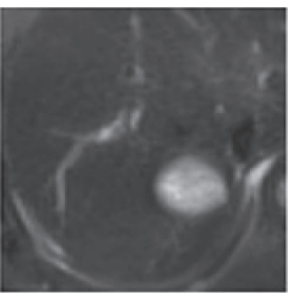

Fig. 1. CT and MR findings (for abstract PS02-020). 


\section{PS02-021 \\ Risk Factors of Recurrence of Hepatocellular Carcinoma after Radiofrequency Ablation: Preliminary Study by Using Early Computed Tomography Evaluation

\author{
Wei Teng ${ }^{1}$, Ka-Wai Liư ${ }^{2}$,Wen-Juei Jeng ${ }^{1}$, Ji-Wei Lin ${ }^{1}$, \\ Wei-Ting Chen ${ }^{1}$, Chen-Chun Lin ${ }^{1}$, Shi-Ming Lin ${ }^{1 *}$ \\ ${ }^{1}$ Department of Gastroenterology and Hepatology, \\ Chang Gung Memorial Hospital, Linkou Branch, \\ Taoyuan, Taiwan; ${ }^{2}$ Department of Radiology, Chang \\ Gung Memorial Hospital, Linkou Branch, Taoyuan, \\ Taiwan
}

Introduction: Recurrence of hepatocellular carcinoma (HCC) remains the major obstacle in curative HCC treatment. The value of immediate computed tomography (CT) evaluation after Radiofrequency ablation treatment (RFA) has not yet clarified. The study aims to investigate whether immediate CT could predict early recurrence in those HCC patients received RFA therapy.

Methods: This retrospective study recruited fresh diagnosed HCC patients who had only single HCC and received RFA in Chang Gung Memorial Hospital, Linkou Medical Center. The diagnosis of HCC was defined by AASLD 2010 guideline by hyperattenuation in the arterial phase with washout in the portal venous phase based on dynamic image, further, all tissue proved by cytology. Immediate follow-up CT defined as image done within 7 days after RFA. Complete ablation was diagnosed as no enhancement of margin by arterial phase and no washout in portal phase. The adequate ablated safety margin detection by follow-up CT defined as $\pi 4 / 3^{*}$ (post RFA CT diameter $+0.5 \mathrm{~cm}$ ) $3 / \pi 4 / 3^{*}$ (pre RFA CT diameter) $3>1$. All patients had received follow-up image regularly every 3 months for the detection of early recurrence.

Results: There were 42 patients (61.9\% male, mean age 70.1 years) were analyzed. Six of them had HCC local recurrence and three of them had distant metastasis after initial complete treatment diagnosed in the following CT scan. The predictive factors of local recurrence are: high AFP level $(\mathrm{P}<$ $0.001)$, artificial ascites or pleural effusion for RFA $(\mathrm{P}=0.027)$ and average safety margin within $0.5 \mathrm{~cm}(\mathrm{P}=0.018)$ are significantly different between two groups.

Conclusion(s): In our study, high AFP level, artificial ascites/pleural effusion and safety margin within $0.5 \mathrm{~cm}$ are predictive of tumor recurrence in post RFA HCC patients. Although complete ablation was proved by early dynamic CT scan (within 7 days), incomplete ablation volume less than average $0.5 \mathrm{~cm}$ safety margin may have tumor recurrence risk. Other images modality such as CEUS may help us evaluate complete ablation in time. Further studies with larger populations are required to validate our findings.

\section{PS02-023 \\ Virological Response to Entecavir Could Not Reduce the Risk of Developing Hepatocellular Carcinoma in Nucleos(t)ide Analogue-Naïve Patients

\author{
Chien-Hung Chen*, Sheng-Nan Lu, Chao-Hung Hung, \\ Jing-Houng Wang, Chuan-Mo Lee, Tsung-Hui Hu \\ Division of Hepato-Gastroenterology, Department of \\ Internal Medicine, Kaohsiung Chang Gung Memorial \\ Hospital, Kaohsiung, Taiwan
}

Introduction: Previous studies demonstrated that virological response (VR) to entecavir is associated with a lower probability of disease progression in chronic hepatitis $\mathrm{B}$ patients. However, the role of VR to entecavir in reducing the risk of hepatocellular carcinoma (HCC) was not investigated in only nucleos(t)ide analogue (NA)-naïve patients.

Methods: To investigate the influence of decompensation cirrhosis and VR to entecavir on HCC development in NA-naïve patients. We investigated 527 chronic hepatitis B patients treated with entecavir therapy for at least 12 months. VR was defined as HBV DNA level $<300$ copies/mL during entecavir therapy.

Results: Of the 527 patients, 39 developed HCC during entecavir treatment. The cumulative probabilities of developing HCC at months 12,36 , and 72 were $0 \%, 0.3 \%$, and $3.5 \%$ for non-cirrhotic patients, respectively; $1.1 \%, 9.2 \%$, and $20.8 \%$ for compensated cirrhotic patients, respectively; and $3.8 \%, 23.8 \%$, and $29.7 \%$ for decompensated cirrhotic patients, respectively $(p<0.001)$. Cox regression analysis showed that cirrhosis and albumin levels at baseline were independent predictors for HCC development. The benefit of VR to entecavir was not a significant factor for HCC development in NA-naïve patients. Furthermore, early achieving VR at month 6 or 12 was also not a significant predictor associated with the development of HCC.

Conclusion(s): Patients with decompensated cirrhosis at baseline had the highest incidence of developing HCC during entecavir therapy. VR to entecavir could not reduce the risk of HCC development in NA-naïve patients. 


\section{PS02-024 \\ Prognostic Factors of Recurred Hepatocellular Carcinoma after Primary Resection}

Jaehong Jeong Nam-Joon Yi*, Kwang-Woong Lee, Kyung-Suk Suh, Suk-Won Suh, Hyeyoung Kim

Surgery, Seoul National University College of Medicine, Seoul, Korea

Introduction: Resection is the treatment of choice for hepatocellular carcinoma (HCC). However, recurrence is still a major problem after primary resection and prognostic factors after recurrence is not well known. So, we investigated prognostic factors of recurred HCC after primary resection to determine who had an aggressive tumor biology.

Methods: We reviewed the patients who underwent surgical resection for HCC between 2005 and 2011. Of a total 941 patients who underwent resection, 589 (62.5\%) patients had recurrences. Among them, 141 patients with extrahepatic recurrences or positive margins were excluded. Finally, we analyzed 448 patients who had intrahepatic recurrences after curative resections.

Results: The median duration between primary resection and recurrence was 9.0 (0.2-90.0) months. At recurrence, 353 (78.8\%) patients had HCCs within Milan's criteria, median level of serum alpha-fetoprotein (AFP) was 13.15 (1.00$427000.00) \mathrm{ng} / \mathrm{ml}$ and median largest tumor size was 1.5 (0.5 12.1) cm. Transarterial chemoembolization (TACE) was performed in 255 (57.0\%) patients for the treatment for recurrence. After recurrence, 5-year disease-free survival rate was $12.8 \%$ and 5-year survival rate was $66.1 \%$. $>5 \mathrm{~cm}$ of primary tumor size, $<1$ year of duration to recurrence, $>200$ of AFP level at recurrence, beyond Milan's criteria at recurrence, and TACE as second-line treatment were revealed as independent poor prognostic factors associated with disease-free survival. Whereas, only Milan's criteria and Child-pugh class at recurrence were identified as independent prognostic factors associated with overall survival.

Conclusion(s): For recurred HCC after primary resection, factors at recurrence including primary tumor size are significantly associated with disease-free survival. Milan's criteria and Child-pugh class at recurrence are associated with overall survival. Therefore, it should be considered when deciding the second-line treatment option for recurred HCC after primary resection.

\section{PS02-025 \\ Pattern Analysis of Recurrent Hepatocellular Carcinoma after Liver Transplantation: MRI Observations \\ Chang-Hee Lee*, Yang Shin Park, Jongmee Lee, Jae Woong Choi, Kyeong Ah Kim, Cheol Min Park \\ Radiology, Korea University Guro Hospital, Seoul, Korea}

Introduction: The only curative options for HCC are surgical resection or liver transplantation (LT). However, recent studies have demonstrated a recurrence in $8-13 \%$ of HCC patient after transplantation. The high incidence of cancer recurrence may reflect poor patient selection. The average time to recurrence ranges from one to two years after LT, and the risk for HCC recurrence depends on tumor size, differentiation, and the presence of vascular involvement. Therefore our aim is to describe the patterns of recurrence and serial MR imaging features of HCC after liver transplantation.

Methods: This retrospective, HIPPA-compliant, institutional review board-approved study was performed with a waiver of informed consent. A database search was performed to identify all cases of recurrent HCC after transplantation during a seven-year period. All patients underwent MRI including precontrast T1, T2-weighted images, and postgadolinium dynamic images. On MRI, we evaluated the characteristics and patterns of recurrent HCC after transplantation.

Results: Seven patients (four men, three women, age range, 45 63; mean 52.7 years) were included in this study. Four patients $(57.1 \%)$ were identified to have a pattern of persistent local disease (PLD), especially near the transplanted liver such as in the inferior vena cava (IVC), hepatorenal space or suture site, within a time period of 2.75 years (range, 2 4 years). Two patients showed recurrent HCC just in the allograft within a time period of five years. One patient showed an intraperitoneal seeding (IPS) pattern which demonstrated diffuse peritoneal infiltration and thickening within a period of nine months. The diffuse metastatic disease (DMD) pattern was observed as a late manifestation of PLD and IPS. Summarizing all cases, the most prominent volume of recurrent tumor burden was found in an extrahepatic (5 of 7 patients) compared to an intrahepatic ( 2 of 7 patients) location. The signal intensities and enhancement patterns did not exhibit change with disease progression.

Conclusion(s): We described 4 patterns of recurrence of HCC following transplant. The most prominent tumor burden was located in an extrahepatic (71.4\%) compared to an intrahepatic $(28.6 \%)$ location. 


\section{Category 3 \\ Surveillance and Diagnosis of Early HCC}

\author{
PS03-001 \\ A Case of Cholangiocarcinoma with a \\ Paraneoplastic Leukemoid Reaction \\ Mimicking a Pyogenic Liver Abscess \\ Hee Yeon Kim${ }^{1}$, Chang Wook Kim ${ }^{1 *}$, Chang Don Lee ${ }^{1}$, \\ Su Lim Lee ${ }^{2}$ \\ ${ }^{1}$ Internal Medicine, The Catholic University of Korea, \\ Seoul, Korea; ${ }^{2}$ Radiology, The Catholic University of \\ Korea, Seoul, Korea
}

Introduction: A paraneoplastic leukemoid reaction is a leukemoid reaction (i.e., leukocytosis $>50,000 / \mu \mathrm{L}$ ) that occurs in association with malignant tumors, not caused by hematological malignancies, infection, or drugs. Cholangiocellular carcinoma (CCC) often mimics hepatic abscesses, and it is more difficult to differentiate CCC presenting as a paraneoplastic leukemoid reaction from a hepatic abscess.

Methods: A 63-year-old male complained of a high fever and progressive generalized weakness.

Results: The laboratory examination showed leukocytosis (leukocytes $22,800 / \mu \mathrm{L}$, neutrophils $78.3 \%$ ), and dynamic enhanced abdominal computed tomography revealed a 3.8-cm rim-enhancing, centrally hypodense lesion in segment 4 of the liver. Initially, the patient was diagnosed with an early liver abscess, and intravenous antibiotics were administered. However, the fever and leukocytosis did not subside, and the hepatic lesion did not mature. An ultrasonography-guided liver biopsy revealed a poorly differentiated adenocarcinoma that stained for cytokeratin-7 and -19, suggestive of CCC. He progressively deteriorated and died 71 days after admission with a peak leukocyte count of $62,000 / \mu \mathrm{L}$.

Conclusion(s): We describe a rare case of CCC presenting with a paraneoplastic leukemoid reaction that was difficult to differentiate from an early liver abscess.

\author{
PS03-005 \\ Feasibility of A-Fetoprotein as a Screening \\ and Diagnostic Tool for Hepatocellular \\ Carcinoma \\ Hyung Joon Kim ${ }^{1 *}$, Jae Cheol Kwon', Dae Geon Ahn', \\ Hyun Woong Lee ${ }^{1}$, Si Hyun Bae ${ }^{2}$, Joon Han Paik ${ }^{3}$ \\ ${ }^{1}$ Internal Medicine, Chung-Ang University College of \\ Medicine, Seoul, Korea; ${ }^{2}$ Internal Medicine, College \\ of Medicine, The Catholic University of Korea, Seoul, \\ Korea; ${ }^{3}$ Internal Medicine, Samsung Medical Center, \\ Sungkyunkwan University School of Medicine, Seoul, \\ Korea
}

Introduction: Recent systematic reviews show that the quality of evidence supporting the use of AFP ( $\alpha$-Fetoprotein) as a diagnostic and screening test for hepatocellular carcinoma (HCC) is limited. The aim of this study was to evaluate the feasibility of AFP as a screening and diagnostic tool for HCC.

Methods: We retrospectively reviewed the medical records of patients with HCC and cirrhosis available in 3 hospitals. 1,560 patients were classified into three groups: 564 were cirrhosis controls, 766 were patients with early stage HCC ( $n=123$ very early, $n=643$ early) and 230 were patients with late stage HCC. The performance of AFP of these three groups was compared each other.

Results: The mean age was 54 years in cases, and 56 years in controls. Male was dominant in both groups (case, 81.1\% vs. control, 65.7\%). The mean AFP was significantly higher in patients with overall HCC (early, late) than in those with liver cirrhosis. [early HCC, $2,769 \mathrm{ng} / \mathrm{ml}(1-200,000)$, late HCC, 9,818 ng/ml (1-639,100), control, 20 ng/ml (0.61$1,753), p<0.0001$ ]. Area under the receiver operating characteristic curve for those overall HCC with cirrhosis was 0.78 . The sensitivity, specificity, and positive predictive value (PPV) of AFP were $52.6 \%, 87.7 \%, 88.3 \%$ at a cutoff of $20 \mathrm{ng} / \mathrm{ml}$; $36.9 \%, 95.9 \%, 94.1 \%$ at a cutoff of $100 \mathrm{ng} / \mathrm{ml}$ and $29.5 \%$, $98.0 \%, 96.3 \%$ at a cutoff of $200 \mathrm{ng} / \mathrm{ml}$. A cutoff of $100 \mathrm{ng} / \mathrm{ml}$ was more sensitive than $200 \mathrm{ng} / \mathrm{ml}$ with equivalent specificity and PPV.

Conclusion(s): Our study cautiously suggested that AFP still has a role in the diagnosis of HCC and the optimal value of AFP in the diagnosis of HCC might be switched $200 \mathrm{ng} / \mathrm{ml}$ to $100 \mathrm{ng} / \mathrm{ml}$. 


\section{PS03-006 \\ Diagnostic Value of Des-Gamma-Carboxy Prothrombin as Hepatocellular Carcinoma Marker Complementary to Alpha-Fetoprotein in China}

\author{
Peipei Song ${ }^{1}$, Xiaobin Feng ${ }^{2}$, Kuansheng $\mathrm{Ma}^{2}$, \\ Norihiro Kokudo ${ }^{1}$, Wei Tang ${ }^{1 *}$ \\ ${ }^{1}$ Hepato-Biliary-Pancreatic Surgery Division, \\ Department of Surgery, Graduate School of Medicine, \\ The University of Tokyo, Tokyo, Japan; ${ }^{2}$ Institute of \\ Hepatobiliary Surgery, Southwest Hospital, Third \\ Military Medical University, Chongqing, China
}

Introduction: Hepatocellular carcinoma (HCC) is the third most common cancer and the second leading cause of cancer-related death for male in the mainland of China. Although remarkable advances have been achieved in the management of HCC in China, most of the HCC patients are not correctly diagnosed until progressed into the advanced stage. Early diagnosis and optimized modality are still the keys for the long-term survival benefit. Currently, the combined assay of des-gamma-carboxyprothrombin (DCP, also known as prothrombin induced by vitamin K absence-II, PIVKA-II) and alpha -fetoprotein (AFP) has been strongly recommended in many studies worldwide to diagnose the patients with HCC (particularly in detection of a small HCC with a high level of sensitivity and specificity). The assay of DCP is approved in many Aisan coutries like Japan, Korea and Indonesia, but it is not used in China yet. Furthermore, differing from Japan and the western countries, the chronic infection of hepatitis $B$ virus (HBV) is the main etiological factor for HCC in China, accounting for $85 \%$ of all cases. In order to estimate the usefulness of DCP for diagnosing HCC in China, we performed the following experiment.

Methods: Serum DCP and AFP levels were determined in 336 HCC cases and 252 non-HCC cases collected in the Institute of Hepatobiliary Surgery, Southwest Hospital, the 3rd Military Medical University. All serum samples were obtained within a week before surgery. Serum DCP levels were measured by electrochemiluminescence immunoassay using a highly sensitive DCP determination kit (ED036, Eisai, Tokyo, Japan) according to the manufacturer's instructions. The HCC cut-off level was set to $>40 \mathrm{mAU} / \mathrm{ml}$.

Results: There was no significant correlation with the serum levels of DCP and AFP. The total sensitivity of DCP was $74 \%(248 / 336)$, the total sensitivity of AFP was $62 \%$ (209/336). The combined testing of DCP and AFP achieved a sensitivity of $84 \%$ (281/336), which was higher than either alone. DCP could achieve a specificity of $56 \%$ with the cut-off value of $40 \mathrm{mAU} / \mathrm{ml}$, and $94 \%$ with the cut-off value of 100 $\mathrm{mAU} / \mathrm{mL}$.

Conclusion(s): No significant correlation was found between serum levels of DCP and AFP. The combined testing of DCP and AFP significantly increased the sensi- tivity over AFP alone. DCP is a useful tumor marker, which plays a complementary role to AFP for the HCC diagnosis in China.

\section{PS03-007 \\ Comparison of CT Angiography with Gadoxetic Acid-Enhanced Magnetic Resonance Imaging in Evaluation of Early and Small Overt Hepatocellular Carcinoma}

Chiah-Yang Chai ${ }^{*}$, Hung Chai Li $i^{2}$, Chia-Cheng Chou', Yei-Sam Hsieh ${ }^{1}$, Shin-Lung Chang ${ }^{3}$, Tun Hui Yang ${ }^{4}$, Chia Hsun Huang ${ }^{5}$

${ }^{1}$ Department of Surgery, Tao-Yuan General Hospital, Ministry of Health and Welfare, Tao-Yuan, Taiwan; ${ }^{2}$ Department of Surgery, Taipei Medical University Hospital, Taipei, Taiwan; ${ }^{3}$ Department of Pathology, Tao-Yuan General Hospital, Ministry of Health and Welfare, Tao-Yuan, Taiwan; ${ }^{4}$ Radiology, Tao-Yuan General Hospital, Ministry of Health and Welfare, TaoYuan, Taiwan; ${ }^{5}$ Gastroenterology, Tao-Yuan General Hospital, Ministry of Health and Welfare, Tao-Yuan, Taiwan

Introduction: Diagnostic discrepancy of early hepatocellular carcinoma occurred between east and west. Intranodular hemodynamic change occurs in multistep human hepatocarcinogenesis. CT angiography (CTHA and CTAP) provides sensitive clue for dual blood flow alteration. Gadoxetic acidenhanced MRI is reported as less invasive alternative with similar result. Diagnostic algorithm of HCC may inflect breakthrough priority following such imaging modality.

Methods: Only small hepatocellular lesions $(<2 \mathrm{~cm})$ in high risk patients who showed atypical imaging attenuation on dynamic study of CT or MRI and conventional hepatic angiography were included in the study. Twenty five lesions received both CT hepatic arteriography and arterioportography. Among these, nine lesions also received gadoxetic acidenhanced MRI (including hepatobiliary phase). The pathological differentiation of the resected specimens were correlated with imaging characteristics.

Results: 3 out of 25 lesions receiving CT angiography were confirmed as early HCC but only one out of the 3 lesions identified showed compatible image result in the gadoxetic acid-enhanced MRI.

Conclusion(s): During hepatocarcinogenesis, most hepatic nodules showed alteration of arterial and portal flow even in very early steps. Gadoxetic acid-enhanced MRI may not detect such lesion. CT angiography is still valuable in predicting the borderline lesions despite its invasiveness. 


\section{PS03-010 \\ AFP, hsAFP-L3 and PIVKA-II for Early Diagnosis of Hepatocellular Carcinoma \\ Do Young Kim ${ }^{1 *}$, Tae Sub Lim¹, Kwang-Hyub Han ${ }^{1}$, Sang Hoon Ahn' ${ }^{1}$, Hyon-Suk Kim² \\ ${ }^{1}$ Internal Medicine, Yonsei University College of Medicine, Seoul, Korea; ${ }^{2}$ Laboratory Medicine, Yonsei University College of Medicine, Seoul, Korea}

Introduction: Early diagnosis of hepatocellular carcinoma (HCC) is very important for a favorable prognosis. Some serologic tests including alpha-fetoprotein (AFP), protein induced by vitamin K absence-II (PIVKA-II), and lens culinaris agglutinin-reactive fraction of AFP (AFP-L3) have been studied as diagnostic markers of HCC; however, there is no consensus on which tumor markers are the most effective in detecting early HCC. In this study, we investigate the clinical utility of tumor markers in the early diagnosis of HCC.

Methods: A total of 669 patients with liver cirrhosis (LC) $(\mathrm{n}=309)$ or HCC $(\mathrm{n}=360)$ were studied from September 2009 to February 2013.

Results: Patients with LC had a mean age of 55.7 years and $64.1 \%$ were male, whereas the mean age of patients with HCC was 58.8 years and $71.4 \%$ were male. We analyzed the expression of tumor markers AFP, PIVKA-II, and AFP-L3 in these patients. All tumor markers were significantly elevated in HCC patients compared with LC patients $(p<0.001)$. The area under the receiver operating characteristic curves (AUROC) of AFP, PIVKA-II, and AFP-L3 for distinguishing HCC from LC was 0.761 (95\% confidence interval [CI], 0.7240.797, p < 0.001), 0.817 (95\% CI, 0.785-0.849, p < 0.001), and 0.749 (95\% CI, 0.712-0.786, p < 0.001), respectively. AFP (AUROC $=0.749,95 \% \mathrm{CI}, 0.686-0.811, \mathrm{p}<0.001$ ) was superior to PIVKA-II (AUROC $=0.693,95 \% \mathrm{CI}, 0.623-0.763, \mathrm{p}<0.001$ ) and AFP-L3 (AUROC $=0.664,95 \%$ CI, 0.453-0.668, $\mathrm{p}<0.001$ ) for diagnosis of early HCC, defined as a single tumor less than $3 \mathrm{~cm}$ in size. The low sensitivity (50.0\%) of AFP (cut-off $20 \mathrm{ng} /$ $\mathrm{ml}$ ) in diagnosis of early HCC can be enhanced by combining it with PIVKA-II (60.0\%). In patients with AFP $<20 \mathrm{ng} / \mathrm{ml}$, the AUROC for PIVKA-II $(0.739,95 \%$ CI, 0.687-0.792; $\mathrm{p}=<0.001)$ was superior to that of AFP-L3 (0.622, 95\% CI, 0.564-0.680; $\mathrm{p}<0.001$ ). AFP-L3 was able to differentiate HCC patients from AFP-false negative patients in the logistic regression analysis (odds ratio 1.054, 95\% CI, 1.030-1.079, $\mathrm{p}=<0.001$ ).

Conclusion(s): In conclusion, combined AFP and PIVKA-II can be used for good screening tool of early HCC. Furthermore, AFP-L3 may have an additional role to differentiate between true HCC in AFP false-positive patients.

\section{PS03-011 \\ Risk Factors for Mortality and Subclassification in Patients with Early Stage Hepatocellular Carcinoma}

Sun Young Yim ${ }^{1}$, Yeon Seok Seo ${ }^{1 *}$, Jae Min Lee ${ }^{1}$, Chang Ho Jung ${ }^{1}$, Eun Sun Kim ${ }^{1}$, Bora Keum¹, Hyunggin An ${ }^{2}$, Yoon Tae Jeen ${ }^{1}$, Hoon Jai Chun ${ }^{1}$, Soon Ho Um ${ }^{1}$, Chang Duck Kim ${ }^{1}$, Ho Sang Ryu', Hong Sik Lee ${ }^{1}$

${ }^{1}$ Department of Internal Medicine, Korea University College of Medicine, Seoul, Korea; ${ }^{2}$ Department of Biostatics, Korea University College of Medicine, Seoul, Korea

Introduction: The prognosis of early stage HCC (BCLC stage 0 or 1 ) is excellent under appropriate treatment, such as surgical resection, RFA, or liver transplantation. However, the population of this early stage HCC is not homogeneous and therefore, subclassification could be useful for predicting prognosis and making decision of treatment plan. This study was performed to evaluate the prognostic factors and to provide subclassification in patients with early stage HCC.

Methods: Patients who were diagnosed as HCC between 2005 and 2013 were enrolled in this study. Patients with previous history of HCC treatment or diagnosed with other malignancies were excluded. Nine-hundred eighty patients fulfilled these criteria. According to the BCLC staging system, 396 (40.4\%), 205 (20.9\%), 260 (26.5\%), and 119 (12.1\%) patients were classified as BCLC stage A, B, C, and D. Among 396 patients with BCLC stage A, 4 patients who had liver transplantation as the first-line treatment and 6 patients who were lost to follow-up within 1 month without any treatment for HCC were excluded.

Results: Age was $60.9 \pm 10.3$ years and 274 patients (71.0\%) were men. Chronic hepatitis B virus infection was the most frequent cause of liver disease (236 patients, $61.1 \%$ ) followed by chronic hepatitis $\mathrm{C}$ virus infection (77 patients, $19.9 \%)$. Child-Pugh score was 5.6 \pm 1.0 and $324(83.9 \%)$ and $62(16.1 \%)$ patients were classified as Child-Pugh grade A and $B$, respectively. Diameter of maximum nodule was $2.3 \pm 0.9 \mathrm{~cm}$ : 299 patients $(77.5 \%)$ had a single nodule, while 87 patients $(22.5 \%)$ had two or three nodules. Serum AFP level was $1.4 \pm 0.9 \log \mathrm{ng} / \mathrm{mL}$. Patients were treated with surgical resection, radiofrequency ablation (RFA), and transarterial treatment in 110 (28.5\%), 218 (56.5\%), and 58 (15.9\%), respectively. Patients were followed up for $31.8 \pm 24.0$ months. During follow-up, 42 patients died and 1-, 3-, and 5-year survival rates were $98.7 \%, 87.2 \%$, and $81.0 \%$, respectively.

On univariate analysis, age ( $<65$ vs $>65$ ), causes of liver disease (HBV vs others), number of nodules (single vs multinodular), up-to-seven score ( $<3.5$ vs $>3.5$ ), UNOS stage (stage 1 vs 2), and Child-Pugh grade (A vs B) were significantly associated with mortality, while diameter of maximum nodule and serum AFP level were not. On multivariate analysis, age $>65$ years, multiple nodules and Child-Pugh grade B were inde- 
pendent risk factors for mortality. The number of risk factors was significantly associated with mortality. Survival duration were $87.2 \pm 1.8$ months, $76.5 \pm 3.3$ months, $57.0 \pm 8.0$ months, and 18.8 \pm 5.6 months in patients with $0,1,2$, and 3 of risk factors, respectively $(\mathrm{P}<0.001)$.

Conclusion(s): The prognosis of patients with early HCC differed according to the age, the number of nodules, and Child-Pugh grade. Application of different treatment strategy according to this subclassification could improve the survival of patients with early HCC.

\section{PS03-012 \\ Growth Rate of Early Stage Hepatocellular Carcinoma in Koreans with Chronic Liver Disease}

\section{Mi-Suk Park*}

Diagnostic Radiology, Severance Hospital, Seoul, Korea

Introduction: The goal of this study was to estimate the growth rate of hepatocellular carcinoma (HCC) and to find host factors that can significantly affect the HCC growth rate.

Methods: A total of 175 patients with early stage HCCs who underwent two or more serial dynamic imaging studies without any anticancer treatment were identified from two tertiary-care hospitals of Korea. For each of these patients, tumor volume doubling time (TVDT) of HCC was calculated by comparing tumor volumes at serial imaging studies, and clinical information and laboratory data were recorded through medical chart reviews.

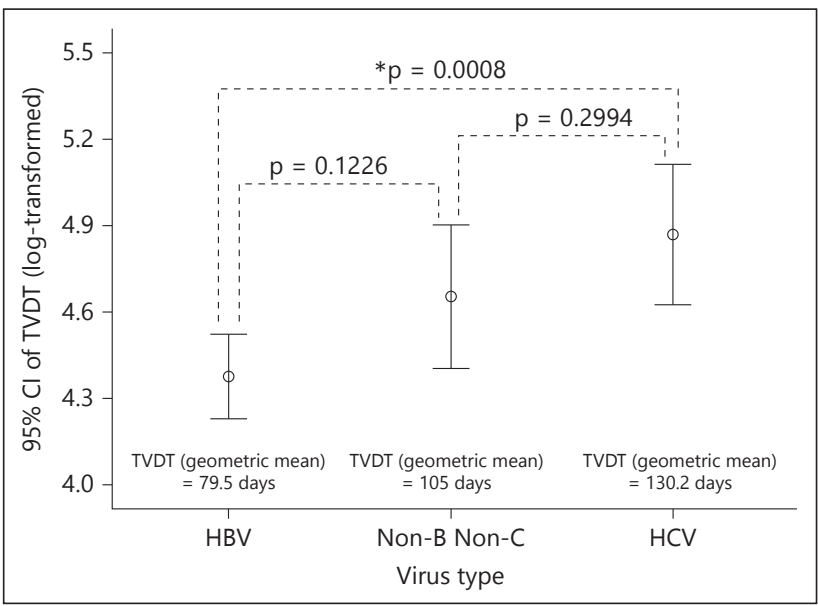

Fig. 1. Difference in Tumor Volume Doubling Time (TVDT) According to Virus Type. HBV, HCV, and Non-B Non-C represent $\mathrm{B}$-viral carriers, C-viral carriers, and non-virus carriers, respectively. $\mathrm{CI}=$ confidence interval. ${ }^{*}$ Statistically significant by ANOVA test followed by post-hoc analysis using Bonferroni correction (for abstract PS03-012).
Results: TVDT ranged from 11 to 851.2 days with a geometric mean of 91.4 days. Multiple linear regression revealed that initial tumor diameter (tumor factor) and etiology of chronic liver disease (host factor) were significantly associated with TVDT. TVDT was shorter when the initial tumor diameter was smaller. Hepatitis B virus (HBV)related HCC showed shorter TVDT than hepatitis C virus (HCV)-related HCC (geometric mean, 79.5 days vs. 130.2 days).

Conclusion(s): Untreated early stage HCC may grow faster in HBV carriers than in HCV carriers in Koreans with chronic liver disease.

\section{PS03-013 \\ The Role of 11C-Acetate and 18f-FDG PET Scan with Contrast CT in Detection of Early HCC - Experience from a Single Liver Transplant Center}

Tan To Cheung ${ }^{1 *}$, Ronnie T.P. Poon ${ }^{1}$, See Chung Chan ${ }^{1}$, Garret C.L. Ho ${ }^{2}$, Kenneth S.H. Chok', Albert C.Y. Chan', Sheung Tat Fan' ${ }^{1}$, Chung Mau Lo ${ }^{1}$

${ }^{1}$ Surgery, The University of Hong Kong, Hong Kong;

${ }^{2}$ Nuclear Medicine, Hong Kong Sanitorium and

Hospital, Hong Kong

Introduction: All of the liver transplantation criteria for HCC depend heavily on tumour size and numbers. However, the sensitivity of contrast CT scan and MRI is far from satisfactory. We investigated whether contrast computed tomography (CT) together with position emission tomography (PET) using two tracers, 11C-acetate and 18F-FDG, provides a more accurate prediction of pathological status by comparing directly to the liver explants.

Methods: Data of 80 HCC patients having the dual-tracer PET together with contrast CT before surgery between January 2004 and October 2013 were reviewed. 26 underwent deceased donor liver transplantation and 54 underwent live donor liver transplantation. All patients had HCC proven by histopathological assessment. Results of HCC staging in terms of the size and number yielded by contrast CT, 18F-FDG PTE and dual-tracer PET with contrast CT were compared with pathological results after surgery.

Results: Total 124 tumours and some 3 patients with uncountable multiple tumors were identified among the 80 patients. The median tumour number were $1(1-4)$ and the median size $(\mathrm{cm})$ was $3.5(0.3-8.0)$ from the final pathology. The overall accurate predication of final pathology in terms of UCSF criteria by contrast CT scan alone was 40/76 (52.6\%), by FDG alone was 19/76 (25.0\%), by C acetate alone 59/76 $(77.6 \%)$ and by overall combination of 3 assessment was $60 / 76(78.9 \%)(\mathrm{p}<0.0001)$.

From the pathological examination, there were 5 tumours $<1 \mathrm{~cm}$, the sensitivity by contrast CT scan alone, by FDG alone, 
by $\mathrm{C}$ acetate alone and by overall combination of 3 assessment were $0 / 9(0 \%), 1 / 9(11.1 \%), 1 / 9(11.1 \%)$ and $1 / 9(11.1 \%)$ respectively $(\mathrm{p}=0.779)$. For tumour range from $1-2 \mathrm{~cm}$, the sensitivity by contrast CT alone, by FDG alone, by C acetate alone and by overall combination of 3 assessment was $22 / 45$ (48.9\%), 4/45 (8.9\%), 31/45 (68.9\%) and 34/45 (75.6\%) respectively $(\mathrm{p}<0.0001)$. For tumour $>2 \mathrm{~cm}$ the sensitivity by contrast CT alone, by FDG alone, by $\mathrm{C}$ acetate alone and by overall combination of 3 assessment was 44/68 (64.7\%), 23/68 (33.8\%), 54/68 (79.4\%) and 59/68 (86.8\%) respectively $(\mathrm{p}<0.0001)$.

Among the study patients, 56 (70.9\%) of them were without FDG uptake and 23 (29.1\%) of them had FDG uptake. The overall survival rates are significantly higher for the no FDG uptake group. The 1-year, 3-year and 5-year overall survival rates for those without FDG uptake versus those with FDG uptake are $98.2 \%, 98.2 \% \& 98.2 \%$ and $94.7 \%, 82.1 \%$ \& $82.1 \%$ respectively $(\mathrm{P}<0.05)$.

Conclusion(s): From this study Dual-tracer PET with contrastCT probably provided the best pathological prediction of explants pathology in patients with HCC up to date. It can be applied selection criteria where number and size of the tumour is mandatory. Its implementation in transplantation work-up should be considered.

\section{PS03-025 \\ The Impact of Serum Alpha-Fetoprotein, Alpha-Fetoprotein L3, and PVIKA II for the Detection of Hepatocellular Carcinoma in the Community in Taiwan}

Yuan-Hung Kuo Sheng-Nan Lu*

Department of Internal Medicine, Kaohsiung Chang Gung Memorial Hospital, Kaohsiung city, Taiwan

Introduction: Background: Hepatocellular carcinoma (HCC), is one of leading causes of malignancy mortality worldwide. Early detection of HCC could lead to a better outcome due to affording more curative therapies. Common tests for HCC surveillance are abdomen ultrasonography (US) examination and serum alpha-fetoprotein (AFP) detection. However, Despite of AFP was not recommended to be a screening tool of HCC in the American Association for Study of Liver Diseases (AASLD) guideline, it is still used as routine in Asia countries. Besides AFP, serum AFP-L3 and protein induced by vitamin $\mathrm{K}$ absence or antagonist II (PIVKA-II) are also used for the detection of HCC in Japan. However, these two markers are not widely used clinically outside Japan.

Aims: To clarify additional effects of AFP-L3 and PIVKA-II than AFP alone in community-based HCC surveillance.

Methods: A county-wide community health screening program for residents aged 40 years and old has also been conducted in 2004 in Tainan. A total of 56702 subjects eligible for the health examination were enrolled. Of them, 286 resi- dents with AFP more than $20 \mathrm{ng} / \mathrm{ml}$ were further received abdomen US. Among these 286 residents, 148 (51.7\%) had stored baseline sera were enrolled into this study, and were further detected their AFP-L3, and PIVKA-II (Wako, Tokyo, Japan) level. HCC patients were identified through US screening, through linkage to the national cancer registry, or from personal history at the baseline screening. The validity of tumor markers associated with HCC diagnosis in community screen was analyzed.

Results: There were 64 male residents and 84 female, with a mean age of 66.3 years. (range: $40 \sim 87.6$ years). The meddle values of AST, ALT, AFP, AFP-L3, PVIKA-II were 72.5 $\mathrm{IU} / \mathrm{ml}, 75 \mathrm{IU} / \mathrm{ml}, 48.8 \mathrm{ng} / \mathrm{ml}, 6.8 \%$ and $5 \mathrm{ng} / \mathrm{ml}$, respectively. Among them, 23 (15.5\%) HCC cases were diagnosed at the baseline screening. Using AFP-L3 to predict HCC, the area under Receiver Operating Characteristic curve (AUROC) was as low as $52 \%, \mathrm{p}=0.757$. With regard to PVIKA-II, the AUROC for HCC prediction was only $51.5 \%, p=0.814$.

Conclusion(s): In community screening, among those subjects with AFP level more than $20 \mathrm{ng} / \mathrm{ml}$, further detection of AFP-L3 or PVIKA-II couldn`t provide sufficient information for HCC identification to save advanced US examinations.

\section{PS03-027 \\ Clinical Characteristics and Outcomes of Hepatocellular Carcinoma in Korea: Results from Random Sample Audit of Statutory Nation-Wide Cancer Registry, 2008-2010}

Bo Hyun Kim ${ }^{1}$, Young-Suk Lim²*, Eun-Yang Kim³, Hyun-Joo Kong ${ }^{3}$, Young-Joo Won ${ }^{3}$, Seung Woon Paik', The Korean Liver Cancer Study Group ${ }^{5}$, The Korea Central Cancer Registry ${ }^{3}$

${ }^{1}$ Center for Liver Cancer, National Cancer Center, Goyang, Korea; ${ }^{2}$ Department of Gastroenterology, Liver center, Asan Medical Center, University of Ulsan College of Medicine, Seoul, Korea; ${ }^{3}$ The Korea Central Cancer Registry, Division of Cancer Registration and Surveillance, National Cancer Center, Goyang, Korea; ${ }^{4}$ Department of Medicine, Samsung Medical Center, Sungkyunkwan University School of Medicine, Seoul, Korea; ${ }^{5}$ The Korean Liver Cancer Study Group, The Korean Liver Cancer Study Group, Seoul, Korea

Introduction: Hepatocellular carcinoma (HCC) is the 5th most common cancer and 2nd cause of cancer mortality in South Korea. The Korea Central Cancer Registry (KCCR) has case completeness $>95 \%$ nationwide, but has limited information about cancer characteristics.

Methods: Out of 35411 HCC registrants to the KCCR between 2008 and 2010, 4962 (14\%) patients were randomly selected and were investigated for clinical characteristics and outcomes through reabstracting audit at 47 hospitals. The data of 4596 patients were analyzed after excluding 366 patients who had insufficient data. 
Results: Mean age of patients was $59 \pm 12$ years and $78 \%$ were males. The causes of HCC were hepatitis B, hepatitis C, and alcohol in $64 \%, 13 \%$, and $32 \%$ of patients, respectively. About $71 \%$ of patients had Child-Pugh class A liver function. The modified UICC stages of patients were I, II, III, IVa, and IVb in $14 \%, 38 \%, 26 \%, 12 \%$, and $10 \%$, respectively. The BCLC stages of patients were $0, A, B, C$, and D in $6 \%, 29 \%, 15 \%, 42 \%$, and $8.3 \%$, respectively. As an initial treatment, transarterial therapy was the most common (46\%), followed by surgical resection (16\%), local ablation (11\%), systemic chemotherapy (4\%), and radiotherapy (1\%). Median overall survival time of the entire patients was 28 months (95\% confidential interval, 25-30 months), and 1- and 3-year survival rates were $62.9 \%$ and $44.6 \%$, respectively.

Conclusion(s): About half of HCC patients are diagnosed at advanced stages, and transarterial therapy are most commonly performed as an initial treatment in Korea. These data from random sample audit of nation-wide HCC registry provide unbiased information of characteristics and outcomes of HCC in Korea and may help establishing public health policy.

\section{PS03-028 \\ Noninvasive Panels for Fibrosis Predict 5-Year Mortality and Development of Hepatocellular Carcinoma in Patients with Chronic Hepatitis B}

\section{Ji Wan Kim, Jeong Han Kim*, Se Woong Kwon, Hyung Min Yu, Hyun Ah Chung, Soon Yong Ko, Won Hyeok Choe, So Young Kwon}

Department of Internal Medicine, Konkuk University School of Medicine, Seoul, Korea

Introduction: The measure of liver fibrosis is an essential step in managing chronic liver disease and evaluating its prognosis. In such patients, the level of fibrosis predicts mortality and liver-related complications. The purpose of this study was to evaluate the degree of liver fibrosis as a predictor of mortality and hepatocellular carcinoma (HCC) development among patients with chronic hepatitis B (CHB).

Methods: Between 2005 and 2006, a total of 542 patients over 18 years old with CHB visited the Konkuk university hospital. We performed noninvasive tests of fibrosis (APRI, FIB-4) and HBV DNA levels. The data on death and new development of HCC collected during a 5-year follow-up was analyzed.

Results: In 5 years, 40 patients died and 60 patients developed HCC. Those who died were significantly older, had lower albumin, lower platelet, and higher FIB-4 than living patients. The same results were observed in patients who newly developed HCC, in comparison to non-HCC patients. The area under the receiver operator characteristic (ROC) curve of APRI, FIB-4, and HBV DNA levels (Log10) for 5 year mortality was $0.731,0.789$, and 0.463 , respectively, with cut points at $0.766,2.671$, and 3.150 , respectively. The area under the ROC curve of these for new development of HCC was 0.760, 0.803 , and 0.523 , respectively, with cut points at $0.766,2.225$, 4.245, respectively. Patients with APRI over 0.766 had a significant risk of death (Odds ratio (OR) $=3.214$, 95\% confidence interval $(\mathrm{CI})=1.009-10.238$ ) and HCC development $(\mathrm{OR}=4.245,95 \% \mathrm{CI}=1.723-10.456)$. Patients with FIB-4 greater than the 2.671 cut point had a significantly higher risk of death (OR $=4.431,95 \% \mathrm{CI}=1.512-12.986)$ and those over the 2.225 cut point had a greater risk of developing HCC (OR = $3.607,95 \% \mathrm{CI}=1.622-8.021$ ).

Conclusion(s): APRI and FIB-4 may be more useful than HBV DNA levels in predicting mortality and development of HCC within 5-years.

\section{PS03-031 \\ Consecutive Increment of Serum AFP Level Is a Useful Surrogate Marker in Predicting HCC in Liver Cirrhosis Patients}

\author{
Hee Chul Nam ${ }^{1}$, Si Hyun Bae ${ }^{1 *}$, Hyun Yang ${ }^{1}$, \\ Hae Lim Lee ${ }^{1}$, Jeong Suk Oh², Ho Jong Chun ${ }^{2}$, \\ Young Seok Kim ${ }^{3}$, Jong Young Choi ${ }^{1}$, Seung Kew Yoon ${ }^{1}$ \\ ${ }^{1}$ Department of Internal Medicine, Seoul St. Mary's \\ Hospital, College of Medicine, The Catholic University \\ of Korea, Seoul, Korea; ${ }^{2}$ Department of Radiology, \\ Seoul St. Mary's Hospital, College of Medicine, The \\ Catholic University of Korea, Seoul, Korea; ${ }^{3}$ Department \\ of Internal Medicine, Soon Chun Hyang University \\ College of Medicine, Seoul, Korea
}

Introduction: The role of alpha-feto protein (AFP) in the diagnosis of hepatocellular carcinoma (HCC) is getting smaller due to the advances of imaging modalities. However, consecutive increment of AFP level in liver cirrhosis patients is presumed to be associated with the higher risk of developing HCC in clinical settings. Such a notion instigated us to analyze serial AFP levels of HCC patients in a retrospective manner.

Methods: From January 2002 to December 2009, 1209 patients were newly diagnosed with HCC in Seoul St. Mary's hospital. Among them 39 patients were found to have a serial record of AFP measurements for one year. We assessed AFP and PIVKAII levels at the time the diagnosis of HCC was made and compared them with that of patients at 1,3,6 and 12 months prior to the diagnosis.

Results: At the time the diagnosis was made, the patients' baseline characteristics were as follows; mean age was 56.76 years (32-87), median tumor size was $3 \mathrm{~cm}$ (0.9-26.3), median AFP level was $698.53 \mathrm{ng} / \mathrm{mL}$ (218.6-32134). Median AFP level of 12 months, 6 months, 3 months and 1 month before the diagnosis of HCC was $13.75 \mathrm{ng} / \mathrm{mL}$ (1.63-513), $51.57 \mathrm{ng} / \mathrm{mL}$ (2.86-1287.86), $146.4 \mathrm{ng} / \mathrm{mL}$ (9.61-1461), $490.38 \mathrm{ng} / \mathrm{mL}$ (107.48-19017), respectively. Repeatedmeasure ANOVA was used to analyze the significance of 
increase in consecutive AFP levels in HCC surveillance. Consecutive increment of AFP level was statistically significant in time dependent manner $(\mathrm{P}=0.001)$ with linear relationship ( $\mathrm{P} \leq 0.000)$. No significant relation was found in that of PIVKAII level.

Conclusion(s): Early detection of HCC with relatively smaller sizes was possible due to the close observation of increase in serial AFP levels. We suggest increase in serial AFP level as a strong surrogate marker in the prediction of HCC and that those with consecutive increments of AFP levels for more than 2 times should be candidates for active surveillances for HCC.

\section{PS03-032 \\ Accuracy of American Association for the Study of Liver Diseases (AASLD) Imaging Diagnostic Criteria in Hepatocellular Carcinoma: A Retrospective Study in a Hepatitis-B Virus (HBV) Endemic Area \\ Hung-Yang Kuo ${ }^{1}$, Chih-Horng $W^{2}{ }^{2}$, Yung-Ming Jeng ${ }^{3}$, Kuo-Hsing Chen ${ }^{4}$, Bang-Bin Chen ${ }^{2}$, Tiffany Ting-Fang Shih ${ }^{2 *}$ \\ ${ }^{1}$ Department of Oncology, National Taiwan University Hospital, Taipei, Taiwan; ${ }^{2}$ Department of Medical Imaging, National Taiwan University Hospital, Taipei, Taiwan; ${ }^{3}$ Department of Pathology, National Taiwan University Hospital, Taipei, Taiwan; ${ }^{4}$ Department of Oncology, National Taiwan University Hospital Yun-Lin Branch, Yun-Lin, Taiwan}

Introduction: Accurate diagnosis of hepatocellular carcinoma (HCC) by non-invasive diagnostic criteria may avoid the risk of hemorrhage-related complications and needle tract tumor seeding following biopsy. Accuracy of AASLD imaging diagnostic criteria for HCC has been validated in Western countries. Sensitivity was reported as 44\%-96\%, and specificity was $96-100 \%$. Its accuracy in HBV endemic Asian countries remains undetermined.

Methods: We used an institutional pathology database to identify all patients (pts) above 20 years old who underwent hepatectomy for primary hepatic tumors from 2010 to 2012. Imaging diagnosis by AASLD diagnostic criteria was made by two radiologists independently. Sensitivity and specificity were calculated after examining clinical risk factors and preoperative imaging reports by AASLD diagnostic criteria and their correlation to pathological diagnoses. Pts with pathological diagnosis of HCC were divided into true-positive (TP) group if they fulfilled AASLD criteria and false-negative (FN) groups if they did not. Clinical information, including age, sex, HBV/HCV status, cirrhosis, tumor size/grade/stage, alphafetoprotein level, was collected to correlate imaging diagnosis and clinical outcome.
Results: Seven hundred twenty three (75.3\%) of the 960 enrolled pts were diagnosed HCC by pathology. The sensitivity and specificity of AASLD criteria were $76.1 \%$ and $92.4 \%$, respectively. Pts in the FN group $(\mathrm{n}=173)$ were older (median age $65 \pm 12$ v.s. $60 \pm 12$ years, $\mathrm{p}<0.05$ ), had fewer HBsAg positivity (27.2\% v.s. $77.5 \%, \mathrm{p}<0.05)$, and less cirrhosis $(14.5 \%$ v.s. $50.4 \%, \mathrm{p}<0.05)$, compared with the TP group $(\mathrm{n}=550)$. Eighty-eight $(50.9 \%)$ pts in FN group had typical imaging findings (arterial phase hypervascularization and portal phase washout), and 46 of them had resolved HBV infection (anti-HBc(+) or anti-HBs(+)). Overall survival was similar between the FN and TP groups (estimated 2-year survival $89.1 \%$ (95\% C.I. $83.8 \%-94.3 \%$ ) v.s. $88.7 \%$ (95\% C.I. $85.6 \%$ $91.8 \%)$.

Conclusion(s): The AASLD diagnostic criteria appeared applicable in Asian patients with resectable hepatic tumors.

\section{PS03-033 \\ IL28B Polymorphism Is an Independent Risk Factor for Hepatitis C-Related Hepatocellular Carcinoma after Antiviral Therapy \\ Tsung-Hui Hu ${ }^{1 *}$, Cheng-Kun Wu ${ }^{2}$ \\ ${ }^{1}$ Hepatologist, Kaohsiung Chang Gung Memorial Hospital, Kaohsiung, Taiwan; ${ }^{2}$ Hepatogastroenterology, Kaohsiung Chang Gung Memorial Hospital, Kaohsiung, Taiwan}

Introduction: Polymorphism at the IL28B gene may predict the therapeutic response and outcome of chronic hepatitis C virus (HCV). However, the impact of IL28B polymorphism on the development of hepatocellular carcinoma (HCC) after peg-interferon and ribavirin therapies (peg-IFN/ RBV) remains controversial.

Methods: We retrospectively test the IL28B polymorphism (rs12979860) in 1118 HCV patients after peg-IFN/RBV therapies.

Results: The frequency of IL28B rs12979860 CC, CT, and TT genotypes in chronic hepatitis C patients was $86.4 \%$, $13.2 \%$, and $0.3 \%$ The duration of follow-up ranged from 6-159 months, with a median of 60 months, 108/1118 (9.66\%) patients had HCC development during follow-up. IL28B CT/TT genotype was positively correlated with baseline AFP levels ( $\geq 20 \mathrm{ng} / \mathrm{ml}$ ), fibrotic stages, diabetes (DM), and sustained virological response (SVR) $(\mathrm{P}<0.05)$. Multivariate Cox's regression analysis showed patients with age $\geq 60$ years, low platelet count, AFP $\geq 20 \mathrm{ng} / \mathrm{ml}$, high fibrotic stage, HCV genotype 1, DM, non-SVR and IL28B CT/TT genotype were significant risk factors for HCC development $(\mathrm{P}<0.05)$. In the subset analysis of SVR patients, although HCC almost occurred in patients with IL-28B CT/TT type, IL-28B was no longer statistically significant for HCC development. In non-SVR patients, the valuable risk factors such as age and diabetes recognized in SVR patients, were no longer significantly risky 
for HCC. However, IL-28B genotypes return to be an important and independent risk factor in non-SVR patients (HR: 1.80, 95\% CI: 1.06-3.07, P = 0.030).

Conclusion(s): IL28B polymorphism seems to be involved in the development of HCV-related HCC, and may be considered as a genetic risk factor for HCV-related carcinogenesis.

\section{PS03-034}

The Autophagy-Related Marker P62 Is Useful for Diagnosis in Human Hepatocellular Carcinoma

Chang Jae Hur ${ }^{1}$, Jung Min Lee ${ }^{1}$, Jeong Min Kim ${ }^{1}$, Hye Min Park ${ }^{1}$, Kyung Ho Yang ${ }^{1}$, Yu Na Kang ${ }^{2}$, Koo Jeong Kang ${ }^{3}$, Woo Jin Chung ${ }^{1}$, Jae Seok Hwang ${ }^{1}$, Byoung Kuk Jang ${ }^{1 *}$

${ }^{1}$ Department of Internal Medicine, Keimyung University School of Medicine, Daegu, Korea; ${ }^{2}$ Department of Pathology, Keimyung University School of Medicine, Daegu, Korea; ${ }^{3}$ Department of Surgery, Keimyung University School of Medicine, Daegu, Korea

Introduction: Several studies showed that accumulation of p62 by impaired autophagy was related with tumorigenesis including HCC. Recent study reported that p62 immunohisto- chemical (IHC) staining can be helpful in the diagnosis of HCC. Therefore, we studied in order to verify the usefulness of p62 IHC staining for pathologic diagnosis of HCC.

Methods: We retrospectively analyzed 186 patients with HCC that was confirmed histologically after complete surgical resection at Keimyung University Dongsan Hospital in Daegu, Korea, from 2001 to 2011. The expression of p62 was analyzed by IHC on HCC and surrounding non-tumor tissues. Sensitivity, specificity and accuracy were evaluated using the chisquare test and McNemar analysis.

IHC expression was evaluated using the proportion score described as the estimated fraction of positively stained tumor cells ( 0 , none; $1,<10 \% ; 2,10-50 \% ; 3,>50 \%)$, and the intensity score representing the estimated staining intensity $(0$, no staining; 1 , weak; 2 , moderate; 3 , strong), and calculating the total IHC staining score equaling the proportion score multiplied by the intensity score. Score 0 was considered as negative, and scores over 0 were considered as positive.

Results: IHC analysis of HCC and surrounding non-tumor tissue showed $85.4 \%$ of sensitivity, $97.5 \%$ of specificity and $85.4 \%$ of accuracy. P62 expression was correlated with Edmonson-Steiner Grades ( $p=0.024)$, however, it was not correlated with TNM stage, BCLC stage, Child-Pugh class, time to recurrence and overall survival period.

Conclusion(s): Our results suggest that the p62 IHC staining can be useful modality for HCC diagnosis. 


\section{Category 4 \\ Controversial Issues in Early HCC}

\author{
PS04-003 \\ Inflammatory and Metabolic Biomarkers and \\ Risk of Liver and Bilary Tract Cancer \\ Liu Lin* \\ Liver Cancer, Jilin University, Beijing, China
}

Introduction: Obesity and associated metabolic disorders have been implicated in liver carcinogenesis; however there is little data on the role of obesity-related biomarkers on liver cancer risk.

Methods: We studied prospectively the association of inflammatory and metabolic biomarkers with risks of hepatocellular carcinoma (HCC), intra-hepatic bile duct (IBD) and gallbladder and bilary tract cancers outside of the liver (GBTC) in a nested case-control study within the European Prospective Investigation into Cancer and Nutrition (EPIC).

Results: Over an average of 7.7 years, 296 participants developed HCC $(n=125)$, GBTC $(n=137)$ or IBD $(n=34)$. Using risk set sampling, controls were selected in a 2:1 ratio and matched for recruitment center, age, sex, fasting status, time of blood collection. Baseline serum concentrations of C-reactive protein (CRP), interleukin-6 (IL-6), C-peptide, total, high-molecular-weight (HMW) adiponectin, leptin, fetuin-a, and glutamatdehydrogenase (GLDH) were measured and incidence rate ratios (IRRs) and 95\% confidence intervals (CI-s) estimated using conditional logistic regression. After adjustment for lifestyle factors, diabetes, hepatitis infection and adiposity measures, higher concentrations of CRP, IL-6, C-peptide and non-HMW adiponectin were associated with higher risk of HCC (IRR per doubling of concentrations = 1.22; $95 \% \mathrm{CI}=1.02-1.46, \mathrm{P}=0.03 ; 1.90 ; 95 \% \mathrm{CI}=1.30-2.77, \mathrm{P}=$ $0.001 ; 2.25 ; 95 \% \mathrm{CI}=1.43-3.54, \mathrm{P}=0.0005$ and $2.09 ; 95 \% \mathrm{CI}=$ 1.19-3.67, $\mathrm{P}=0.01$, respectively).

Conclusion(s): CRP was associated also with risk of GBTC $(\mathrm{IRR}=1.22 ; 95 \% \mathrm{CI}=1.05-1.42, \mathrm{P}=0.01) . \mathrm{GLDH}$ was associated with risks of HCC $(\mathrm{IRR}=1.62 ; 95 \% \mathrm{CI}=1.25-2.11, \mathrm{P}=$ $0.0003)$ and $\operatorname{IBD}(\mathrm{IRR}=10.5 ; 95 \% \mathrm{CI}=2.20-50.90, \mathrm{P}=0.003)$. The continuous net reclassification index was 0.63 for CRP, IL-6, C-peptide and non-HMW adiponectin, and 0.46 for GLDH indicating good predictive ability of these biomarkers. Conclusion: Elevated levels of biomarkers of inflammation and hyperinsulinemia are associated with a higher risk of HCC, independent of obesity and established liver cancer risk factors. (Hepatology 2014;). \n\nCopyright (C) 2014 American Association for the Study of Liver Diseases.

\section{PS04-004 \\ Stereotactic Ablative Radiotherapy Combined to Incomplete Transcatheter Arterial Chemoembolization in Unresectable Hepatocellular Carcinoma}

\begin{abstract}
Eun Kyung Paik ${ }^{1}$, Mi-Sook Kim ${ }^{1 *}$, Won Il Jang ${ }^{1}$, Young Seok Seo", Chul-Koo Cho ${ }^{1}$, Hyung Jun Yoo", Chul Ju Han', Su Cheol Park2, Sang Bum Kim³, Young Han Kim ${ }^{4}$

${ }^{1}$ Radiation Oncology, Korea Institute of Radiological and Medical Sciences, Seoul, Korea; ${ }^{2}$ Internal Medicine, Korea Institute of Radiological and Medical Sciences, Seoul, Korea; ${ }^{3}$ Surgery, Korea Institute of Radiological and Medical Sciences, Seoul, Korea; ${ }^{4}$ Radiology, Korea Institute of Radiological and Medical Sciences, Seoul, Korea
\end{abstract}

Introduction: The treatment of choice for hepatocellular carcinoma (HCC) is surgery, but less than $20 \%$ of the patients with HCC are suitable for surgery. Currently, transcatheter arterial chemoembolization (TACE) is widely conducted for patients with early stage HCCs that are unresectable or unable to be treated with local ablative therapies. However, TACE alone rarely produces a complete response, and additional treatment is required. Although, various treatment modalities such as radiofrequency ablation (RFA), percutaneous ethanol injection (PEI), sorafenib, three-dimensional conformal radiotherapy (3D-CRT), and stereotactic ablative radiotherapy (SABR) have been suggested in addition to TACE, no clear guideline has yet been established. Therefore, this study was designed to evaluate the effect of combining SABR after incomplete TACE.

Methods: We retrospectively studied 178 patients treated with TACE as initial therapy for HCC between 2006 and 2011, who satisfied the following eligibility criteria, which are also the criteria used at our institute for SABR with curative aim in HCC patients. The inclusion criteria: (1) Barcelona Clinic Liver Cancer (BCLC) stage 0 or A, (2) single or multiple lesions up to three nodules, (3) each tumor size $\leq 10 \mathrm{~cm}$, with total sum $\leq 10 \mathrm{~cm}$, (4) Child-Turcotte-Pugh (CTP) score $\leq 7$, (5) without major vessel invasion, and (6) without extrahepatic metastases. The exclusion criteria were: (1) diffuse infiltrative tumor type, (2) liver cirrhosisassociated complications, (3) severe co-morbidities, (4) previous radiotherapy to the upper abdomen, and (5) other malignancies within 5 years. Survival was calculated from the date of the initial TACE using the Kaplan-Meier method and intergroup comparisons were performed using the log-rank test. 
Results: After receiving TACE as a primary treatment, 24 patients showed complete response to TACE (Group 1). In the incomplete response group, 47 patients were further treated with curative treatments (Group 2) such as surgery, RFA, or PEI, 37 patients with SABR (Group 3) with a total dose of 40-60 Gy (median 56 Gy) in 3-5 fractions, and 70 patients with non-curative treatments (Group 4) such as repeated TACE, sorafenib, or chemotherapy. The characteristics of patients among Groups 1, 2, and 3 did not show any significant differences from each other. There were also no significant differences between Groups 3 and 4. However, tumors in Group 1 tended to have lower BCLC stage and smaller size, and tumors in Group 2 tended to have lower CTP score and smaller size. The 2 year overall survival (OS) rates for Group $1,2,3$, and 4 were $88 \%, 81 \%, 73 \%$, and $54 \%$, respectively. The 5 year OS rates for Group 1, 2, 3, and 4 were 50\%, 58\%, $53 \%$, and $28 \%$, respectively. The OS rates did not show significant differences among Groups 1, 2, and 3. Only group 4 showed significantly lower survival rates than the other three groups.

Conclusion(s): The addition of SABR after incomplete TACE showed significant survival benefit over additional noncurative treatments. Moreover, it also showed similar survival outcomes as the good prognostic groups, such as the group showing complete response to TACE and the group combining curative treatments with incomplete TACE. Therefore, if patients were indicated for SABR at initial diagnosis, SABR may be recommended after failure of TACE.

\section{PS04-006 \\ Long-Term Survival for Patients with Solitary Hepatocellular Carcinoma Not More Than $\mathbf{2}$ cm after Radiofrequency Ablation: Thrombocytopenia Does Not Associate with Poor Survival \\ Chen-Chun Lin*, Shi-Ming Lin, Wei-Ting Chen, \\ Ji-Wei Lin, Chen-Chih Liao, Wei Teng \\ Division of Hepatology, Liver Research Unit, Department of Gastroenterology and Hepatology, Linkou Chang Gung Memorial Hospital, Taipei, Taiwan}

Introduction: Surgical resection for hepatocellular carcinoma (HCC) $<2 \mathrm{~cm}$ has a 5-year survival as high as $70 \%$ (Arii, et al., Hepatology, 2000 and Roayaie, et al., Hepatology,
2013). However, the rates decrease to $57-60 \%$ if their platelet counts $<150,000 /$ uL. Radiofrequency ablation (RFA) could effectively treat HCC $<2 \mathrm{~cm}$ and have similar survival to surgical resection (Wang, et al. J Hepatol, 2012). But the prognosis of thrombocytopenia for $\mathrm{HCC}<2 \mathrm{~cm}$ after RFA treatment is still unknown. This study wants to look for the independent factors of HCC $<2 \mathrm{~cm}$ after RFA and to explore the outcomes of patients with thrombocytopenia.

Methods: The study included 186 patients between 2004 and 2012 with a mean follow-up of 37.7 months in Linkou Chang Gung Memorial Hospital. All were newly diagnosed as solitary HCC $<2 \mathrm{~cm}$ proven by cytology or pathology and received RFA as the primary treatment. Pretreatment data showed age $>70$ years (36.6\%), HBV (44.1\%), HCV (50.0\%), Child-Pugh A/B/C (83.3\%/12.9\%/2.7\%), mean tumor size $(16.9 \pm 2.2 \mathrm{~mm})$, median platelet count $(108,500 \pm 53,012 / \mathrm{uL})$ and cirrhosis (91.4\%).

Results: A total of 177 ( $95.2 \%$ ) patients achieved primary technique effectiveness after $1.08 \pm 0.27$ courses of RFA. Overall 5-year survival was $65.4 \%$. The 90-day mortality occurred in one $(0.5 \%)$ patient with Child-Pugh C and platelet count less than 100,000/uL. Univariate analysis showed age, serum AFP level, histocytological grading, creatinine and Child-Pugh class were association with survival. Multivariate analysis by Cox regression disclosed Child-Pugh class and creatinine independently predicted survival. Patients with thrombocytopenia had a similar 5-year survival to those without thrombocytopenia (platelet count $<150,000 / \mathrm{uL}$ vs $>$ $150,000 /$ uL, $63.4 \%$ vs. $64.3 \%, \mathrm{P}=0.611$; platelet count $<100,000 / \mathrm{uL}$ vs $>100,000 / \mathrm{uL}, 61.3 \%$ vs $68.8 \%, \mathrm{P}=0.456)$. In the subgroups of Child-Pugh A and platelet count $<100,000 /$ $\mathrm{uL}$, the 5-year survival could achieve $72.1 \%$. Major complications occurred in $4(2.2 \%)$ patients. All the 4 patients had platelet count $<150,000 / \mathrm{uL}$.

Conclusion(s): Radiofrequency ablation could achieve a high 5-year survival rate for patients with $\mathrm{HCC}<2 \mathrm{~cm}$. Patients with low platelet count but good liver reserve still have excellent outcomes after RFA. 


\section{Category 5 \\ Curative Treatment by Surgery}

\author{
PS05-001 \\ Meaning of Hepatic Venous Pressure \\ Gradient in Patients with Hepatocellular \\ Carcinoma \\ Jae Sik Chung ${ }^{1}$, Sung Hoon Kim ${ }^{1 *}$, Moon Young Kim², \\ Kyung Sik Kim ${ }^{3}$, Soon Koo Baik ${ }^{2}$ \\ ${ }^{1}$ Department of Surgery, Yonsei University Wonju \\ College of Medicine, Wonju, Korea; ${ }^{2}$ Department of \\ Internal Medicine, Yonsei University Wonju College \\ of Medicine, Wonju, Korea; ${ }^{3}$ Department of Surgery, \\ Yonsei University College of Medicine, Seoul, Korea
}

Introduction: Hepatic venous pressure gradient (HVPG) was introduced as important prognostic marker in patients with hepatocellular carcinoma underwent liver resection. However, this procedure did not performed routinely due to invasive technique. This study investigated the meaning of pretreatment hepatic venous pressure gradient and compared HVPG with other non-invasive and indirect markers of portal hypertension.

Methods: We prospectively measured HVPG in patients with cirrhosis. After HVPG measurement, 29 patients with hepatocellular carcinoma underwent treatment were enrolled in this study. We identified short-term outcomes according to the HVPG and clinical portal hypertension and analyzed correlation between HVPG and other markers. Additionally, we investigated the prognostic factors for high grade complication.

Results: Median HVPG was $11 \mathrm{~mm} \mathrm{Hg}$ (range 4-28) and fibroscan result was $19.5 \mathrm{k} \mathrm{kPa}(4.1-75)$. Twenty one patients showed evidence of clinical portal hypertension. Eight, Eight and twelve patients underwent liver resection, radiofrequency ablation (RFA) and transcatheteric arterial chemoembolization (TACE), respectively. Two, seven and twelve patients showed HVPG more than $10 \mathrm{mmHg}$ in liver resection, RFA and TACE group, respectively. High grade complications were only developed in RFA and TACE group. Treatment modality was not prognostic factor for high grade complication. The only esophageal varix was found as significant prognostic factor in multivariate analysis.

Conclusion(s): Although our study has a limitation of small case number, curative treatment modalities including liver resection and RFA may be applied to patients with portal hypertension who did not develop esophageal varices.

\author{
PS05-002 \\ Effects of Locoregional Treatments before \\ Living Donor Liver Transplantation on \\ Overall Survival and Disease-Free Survival \\ Gun Hyung Na, Dong Goo Kim*, Soo Ho Lee, \\ Eun Young Kim, Tae Ho Hong, Young Kyoung You \\ Department of Surgery, Seoul St. Mary's Hospital, \\ The Catholic University of Korea, Seoul, Korea
}

Introduction: We evaluated the effects of pre-transplant locoregional treatment on survival in living donor liver transplantation (LDLT), the survival after LDLT in successfully down-staged patients, and the most accurate method for predicting survival after LDLT.

Methods: From December 2003 to December 2012, 130 patients who were newly diagnosed with hepatocellular carcinoma (HCC) at our hospital underwent LDLT for HCC at our transplant center. Pre-transplant locoregional treatments for HCC were performed in 86 (66.2\%) patients.

Results: Disease-free survival (DFS) and overall survival (OS) after LDLT, as well as the intention to treat survival after HCC diagnosis in the non-pre-transplant locoregional treatment group, were better than those in the pre-transplant locoregional treatment group. Of the 33 patients with HCC initially beyond the Milan criteria, 12 (36.4\%) experienced successful down-staging after locoregional treatments, and the 5-year DFS and OS were 81.8 and $75.0 \%$, respectively, which was comparable to those in patients with HCC initially within the Milan criteria. A bad responder according to the modified Response Evaluation Criteria in Solid Tumors (mRECIST) $(\mathrm{p}=0.042)$, and increased alpha-fetoprotein (AFP) levels $(p=0.004)$ during pre-transplant locoregional treatments were independent risk factors for HCC recurrence after LDLT in multivariate analysis.

Conclusion(s): Although patients in the non-pre-transplant locoregional treatment group had a better outcome, more research is needed in LDLT. Liver transplantation may be considered after successful down-staging in patients with HCC initially beyond the Milan criteria, and mRECIST and serum AFP level changes are better selection criteria for LDLT in patients who have received locoregional treatments. 


\section{PS05-003 \\ Virological Response to Anti-Viral Therapy Predicts the Recurrence of HBV-Related HCC after Curative Resection}

Won Sohn, Ju Yeon Cho, Jemma Ahn, Dong Hyun Sinn, Geum-Youn Gwak, Moon Seok Choi, Joon Hyeok Lee, Kwang Cheol Koh, Seung Woon Paik, Byung Chul Yoo, Yong-Han Paik*

Gastroenterology, Samsung Medical Center, Seoul, Korea

Introduction: Recent studies have shown that anti-viral therapy may reduce the recurrence of hepatocellular carcinoma (HCC) in patients with hepatitis B virus (HBV). The aim of this study was to investigate the effect of virological response to anti-viral therapy on the recurrence after curative resection in patients with HBV-related HCC.

Methods: From January 2008 to December 2010, a total of 72 anti-viral therapy naïve patients underwent curative resection for HBV-related HCC (single nodule; $<5 \mathrm{~cm}$ in diameter, or multi-nodule; number $\leq 3$ and diameter $<3 \mathrm{~cm}$ ). All patients were treated with anti-viral therapy within 1 month after resection (entecavir, 58; clevudine, 11; lamivudine, 3 patients). We assessed the predictive factors for recurrence of HCC after curative resection. Complete response to anti-viral therapy was defined as an undetectable state in HBV DNA test using real-time PCR analysis.

Results: The median follow-up duration was 41.7 months. Cumulative recurrence rates after resection at 1,3 , and 5 years were $14.0 \%, 34.2 \%$, and $45.0 \%$, respectively. While 45 patients had complete virological response to antiviral therapy at 6 months after resection, 27 patients had incomplete virologiacal response. The multivariate analysis showed that risk factors for recurrence were the multinodularity (hazard ratio (HR) 8.27, $\mathrm{p}=0.001$ ), presence of microvascular invasion (HR 2.92, $\mathrm{p}=0.006$ ), and incomplete virological response to anti-viral therapy (HR 2.98, p = 0.009).

Conclusion(s): Virological response to anti-viral therapy was associated with the recurrence of after curative resection in patients with HBV-related HCC. This study suggests that active suppression of viral load can prevent the recurrence of HCC after resection.

\section{PS05-005 \\ Treatment of HCC Meeting the Milan Criteria by Living Donor Liver Transplantation or Liver Resection}

Kuan-Chun Hsueh ${ }^{1}$, Teng-Wei Chen ${ }^{2}$, Shung-Sheng Tsou', Tzu-Ming Chang ${ }^{1}$, Chew-Teng Kor ${ }^{3}$, Chung-Bao Hsieh ${ }^{2 *}$

${ }^{1}$ Division of General Surgery, Department of Surgery, Tungs' Taichung MetroHarbour Hospital, Taichung, Taiwan; ${ }^{2}$ Division of General Surgery, Department of Surgery, Tri-Service General Hospital, National Defense Medical Center, Taipei, Taiwan; ${ }^{3}$ Cancer Center, Tungs' Taichung MetroHarbour Hospital, Taichung, Taiwan

Introduction: Liver resection (LR) and liver transplantation (LT) are considered curative treatments for early hepatocellular carcinoma (HCC). Owing to the shortage of donor organs, living donor liver transplantation (LDLT) has been administered widely in recent years. This study investigated the survival outcome of patients who were diagnosed of HCC conforming Milan criteria after LR or LDLT. Patients who underwent LR may have worse prognosis because of the liver failure or recurrences outside the Milan criteria, which resulted in the unavailability of performing salvage LT to obtain better prognosis. We would like to know the risk factors related to the poorer outcome to identify what patients could benefit from LT.

Methods: Between January 2006 and January 2013, 229 patients who were under the age of 70 and had been diagnosed of HCC that met the Milan criteria in Tri-Service General Hospital and Tungs' Taichung Metroharbor Hospital underwent LR ( $\mathrm{n}=191)$ or living donor liver transplantation (LDLT) $(\mathrm{n}=38)$. The overall survival (OS) and disease-free survival (DFS) were found through propensity score analysis and one-to-two matching between these two study groups. The factors affecting associated with poor clinical results for patients after LR were analyzed by multi-variate Cox regression model. Furthermore, the risk stratification of these factors for these patients was performed.

Results: In the propensity model, patients treated by LDLT had relatively poorer liver functions than those treated by LR. The 1-, 3-, 5-year OS rates were 94.1\%, 79.6\%, 79.6\% for LDLT and $94.3 \%, 80.2 \%, 74.3 \%$ for LR, respectively $\mathrm{p}=$ 0.858). However, it seemed that LDLT could offer better OS than LR for these patients simply 4 years after the procedure. The 1-, 3-, 5-year DFS rates were $91.1 \%, 74.8 \%, 74.8 \%$ for LDLT, and $77.1 \%, 55.1 \%, 36.8 \%$ for LR, respectively ( $\mathrm{p}=$ $0.005)$. On multivariate analysis, presence of F4 cirrhosis of nontumorous liver parenchyma, microvascular invasion and multiplicity of tumors were associated with poor results after LR and were assigned as risk point 2, 1 and 1, which were weighted though multiple logistic regression. By risk stratification analysis, patients who scored 4 were classified as highrisk group and had $77.81 \%$ of risk of developing liver failure or tumor recurrences outside the Milan criteria. 
Conclusion(s): No significant difference in 5-year survival rate between LDLT and LR in patients with HCC fulfilling the Milan criteria, despite it favored LDLT merely 4 years after the operation. The DFS was significantly better for patients who were treated by LDLT. For HCCs meeting the Milan criteria but having highest risk scores, primary or preemptive LDLT could be regarded as treatment of choice.

\section{PS05-006}

Advanced Age Is Not a Contraindication for Liver Resection in Cases of Large Hepatocellular Carcinoma

Hsiu-Lung Fan Teng-Wei Chen, Chung-Bao Hsieh*

General Surgery, Tri-Service General Hospital, Taipei, Taiwan

Introduction: The role of surgery in the management of large hepatocellular carcinomas (HCCs) is controversial. Advanced age and comorbidities are taken into account when major surgery is considered.
Methods: This study included 70 patients aged 70 years or older treated for large HCCs ( $\geq 5 \mathrm{~cm}$ ) between January 2007 and December 2012: 37 underwent LR and 33 underwent TACE. The outcomes of these patients were retrospectively analyzed. Univariate and multivariate Cox proportional hazard models were established. Kaplan-Meier survival curves were generated, and survival data were compared using the log-rank test.

Results: Hospital stay was significantly longer in the LR group than in the TACE group ( 10 days vs 8.5 days; $\mathrm{P}=0.003$ ). Treatment-related complications were more frequent in the TACE group, but this difference was not statistically significant. LR was associated with a better disease-free survival rate, median survival rate and cumulative overall survival rate.

Conclusion(s): Our results showed that LR could be a safe and effective treatment option for HCC tumors $\geq 5 \mathrm{~cm}$ in patiets aged 70 years or older. 


\section{Category 6 \\ Current Understanding of the Biology of HCC}

\author{
PS06-003 \\ Introduction of Taiwan Liver Cancer Network \\ Shiu-Feng Huang ${ }^{1 *}$, Chi-Ling Chen ${ }^{2}$, Tseng-Chang Yen ${ }^{3}$, \\ Pei-Jer Chen'2, Yun-Fan Liaw ${ }^{4}$ \\ ${ }^{1}$ Institute of Molecular and Genomic Medicine, \\ National Health Research Institutes, Miaoli, Taiwan; \\ ${ }^{2}$ Graduate Institute of Clinical Medicine, National \\ Taiwan University, Taipei, Taiwan; ${ }^{3}$ Department of \\ Applied Mathematics and Institute of Statistics, \\ National Chung-Hsing University, Taichung, Taiwan; \\ ${ }^{4}$ Liver Research Unit, Chang Gung Memorial Hospital, \\ Taoyuan, Taiwan
}

Introduction: Recent advances in genomics, proteomics, and increasing demands for biomarker validation studies have changed the landscape of cancer research. The establishment of tissue bank for translational research became very important. Hepatocellular carcinoma (HCC) is the number one of the ten leading cancer death in Taiwan. Taiwan Liver Cancer Network (TLCN) was organized by government fund (NRPGM) in year 2005 that coordinated five major medical centers in Taiwan to collect tumor tissues and blood biosamples of liver tumor patients along with comprehensive clinical and epidemiological data. The five medical centers are located in the northern (National Taiwan University Hospital and Chang Gung Memorial Hospital Linko Branch), central (Taichung Veteran General Hospital), and southern parts (Chang Gung Memorial Hospital Kaohsiung Branch and Kaohsiung Veteran General Hospital) of Taiwan. In this way, this network would be able to recruit liver cancer patients with various socioeconomic, ethnical and life style backgrounds as well as regional representativeness.

Methods: A team consists of hepatologist, surgeon and pathologist was formed in each collaborating medical center to provide these materials and clinical information. A welltrained research nurse was provided by the project and stationed at each collaborating hospital to help with all the collection procedures. All participating centers must follow a common protocol to collect biosamples as well as the patients' clinical, pathological and epidemiological information. Standardized questionnaire and clinical abstract were designed prospectively and used for all patients recruited in TLCN. The HCC and non-tumor tissues from resection and blood samples were frozen and stored in deep freezer at each collaborating hospital first and shipped to the central tissue bank at National Health Research Institutes (NHRI) monthly. There was a database core to be in charge of the data management. A User Committee composed of ten famous scientists in liver research was organized to review the applications for biosamples.
Results: This network started to collect biosamples since October, 2005. By Feb. 30, 2013, we have successfully collected more than 7,000 liver cancer patients with tumor tissue or blood samples and sufficient clinical and pathological information. Among them, 85\% were HCC patients. Since 2007, this network has started to accept applications for biosamples, and associated clinical data, from individual investigators in Taiwan. So far, we already have 87 applications, and have sent out more than 30,500 biosamples. Our Applicants have published 31 papers.

Conclusion(s): The goal of TLCN was to provide high quality biosamples with reliable and sufficient clinical information for scientists in Taiwan to facilitate the liver cancer research and achieve break though in HCC treatment and prevention. We have become the major resource of HCC research in Taiwan, which is one of our major goals. Our applicants have performed successful research and have very good publications. Most of those research papers are deal with molecular markers related to treatments or invasiveness. They should have contribution to the improvement for HCC outcome in the near future.

\section{PS06-004 \\ Plectin Deficiency Promotes Cancer Cell Motility in Human Hepatocellular Carcinoma \\ Chiung-Chi Cheng ${ }^{1}$, Yih-Shyong Lai ${ }^{1 *}$, Yi-Hsiang Liu', Yen-Chang Clark Lai ${ }^{2}$ \\ ${ }^{1}$ Department of Pathology, Bing Show Chwan Memorial Hospital, Changhua, Taiwan; ${ }^{2}$ Department of Pathology, Kaohsiung Medical University Chung-Ho Memorial Hospital, Kaohsiung, Taiwan}

Introduction: Cytolinker proteins play a crucial role in cellular architecture. As a modulator of cytoskeletal organization and scaffolding platform, plectin have been reported to affect cellular signaling and activities such as stress response, migration, polarization, and actin filaments dynamic. Plectin is mainly located at hemidesmosomes (connected with integrin $\alpha 6 \beta 4$ ), desmosomes, Z-line in smooth muscle and branching point of intermediate filaments (IFs). Previous studies revealed that alteration of cytokeratin 18 (CK18) due to plectin deficiency in hepatocellular carcinoma (HCC). In epithelial cells, cytokeratin are connected by plectin to the integrin $\alpha 6 \beta 4$ at hemidesmosomes serves as an anchorage between cell and matrix. Plectin deficiency in HCC may affect the disassembly and assembly of hemidesmosome and further alter cell motility during wound healing, differentiation or 
The 5th Asia-Pacific Primary Liver Cancer Expert Meeting (APPLE 2014)

cancer metastasis. Hence, we proposed a hypothesis that whether plectin deficiency in HCC modulate cell motility

Methods: From transwell migration assay, plectin siRNA knockdowned Chang cell also showed a higher relative migration. Moreover, we observed that plectin knockdowned Chang cells were less organized, more polarized, more focal adhesion kinase (FAK) activity compared to mock Chang cell by confocal microscopy.

Results: Our preliminary result also demostrated that plectin siRNA knockdowned Chang cell showed a higher relative Rac1-GTPase activity by using Rac1 (Ras-related C3 botulinum toxin substrate 1) pull down assay. From immunohistochemical assay on human HCCs, FAK of invasive front of tumor is highly expressed.

Conclusion(s): From these information, we suggested that plectin deficiency in human HCC modulate higher cancer cell motility.

\section{PS06-005 \\ Emodin Attenuate Radioresistance in Hypoxic Human Hepatoma Cell by Inhibition of Epigenetic Signaling such as Histone Demethylase JMJD2B}

Sang Youn Hwang ${ }^{1 *}$, Seon Mi Lee ${ }^{2}$, Joon Suk Kim', Jung Woo $\mathrm{Im}^{2}$, Cheol Won Choi ${ }^{3}$, Gwang Mo Yang ${ }^{3}$, Kyu $\mathrm{Heo}^{4}$

${ }^{1}$ Internal Medicine, Dongnam Institute of Radiological \& Medical Sciences, Busan, Korea; ${ }^{2}$ Internal Medicine, Dongnam Institute, Busan, Korea; ${ }^{3}$ Radiation Oncology, Dongnam Institute, Busan, Korea; ${ }^{4}$ Research Center, Dongnam Institute, Busan, Korea

Introduction: HCC itself can be frequently exposed to hypoxia associated with rapid growth and anti-angiogenic treatment such as TACE or sorafenib. Hypoxia induces radioresistance by interfering with the fixation of DNA damage and activating several hypoxia induced signals such as hypoxia inducible factor- 1 alpha (HIF-1 $\alpha$ ) and histone demethylase etc. Emodin (1,3,8-trihydroxy-6-methylanthraquinone), a family of plant derived polyphenol has been proven to have anticancer properties. There is limited data about role of emodin for epigenetic mechanism in hypoxic human hepatoma cell line. In this study, we examined the followings: (i) whether emodin attenuated radioresitance induced by hypoxia in human hepatoma cell line, (ii) what was the epigenetic mechanism of radiosensitization.

Methods: One human HCC cell line (HepG2) were used in this study. They were exposed to four different manners in environmental hypoxia (1\% 02) and normoxia; none (control), irradiation (10Gy, one fraction), emodin $(25 \mu \mathrm{M})$, and irradiation combined with emodin. Cells were then subjected to MTT assay (3-(4,5-Dimethylthiazol-2-yl)-2,5-diphenyltetra- zolium bromide, a yellow tetrazole) and immunoblotting in 24 hours and 72 hours after exposure.

Results: The proliferation of HepG2 cells was significantly suppressed in hypoxia and normoxia as following order: combination group > irradation only > emodin only. Combination of irradiation and emodin maximally downregulated HIF-1 $\alpha$ and histone demethylase JMJD2B.

Conclusion(s): Emodin may attenuated radioresistance induced by hypoxia in HepG2 via down-regulation of hypoxia induced epigenetic signal such as histone demethylase JMJD2B. Therefore, our findings may provide new insights into understanding epigenetic mechanism of emodin as radiosensitizer in hypoxia HCC.

\section{PS06-006 \\ Variants of HLA-DQ Locus and Stat4 Are Associated with Hepatocellular Carcinoma in Asian Patients with Chronic Hepatitis B Infection \\ Tai-Chung Tseng ${ }^{1}$, Chun-Jen Liu ${ }^{2}$, Wan-Ting Yang ${ }^{3}$, Ding-Shinn Chen ${ }^{3}$, Jia-Horng Kao ${ }^{3 *}$ \\ ${ }^{1}$ Department of Internal Medicine, Taipei Tzuchi Hospital, The Buddhist Tzuchi Medical Foundation, Taipei, Taiwan; ${ }^{2}$ Master of Public Health Degree Program, National Taiwan University, Taipei, Taiwan; ${ }^{3}$ Department of Internal Medicine, National Taiwan University Hospital, Taipei, Taiwan}

Introduction: Hepatocellular carcinoma (HCC) is the most lethal complication of patients with chronic hepatitis B infection. Viral genomic risk factors have been explored extensively, but host genetic factors have not been well explored.

Methods: We collected 706 patients with HBV-related HCC as cases and 805 HBV inactive carriers as controls. We genotyped 3 candidate single nucleotide polymorphisms (SNP) identified from previous genome-wide association studies, including rs9275319 at HLA-DQ region, rs7574865 at STAT4 and rs17401966 at KIF1B. The association between the SNPs and HCC was then analyzed.

Results: The distribution of all three SNPS did not violate Hardy-Weinberg equilibrium. We found that rs9275319 and rs7574865, but not rs17401966, were associated with HCC development. Using additive model, the minor alleles of rs9275319 and rs7574865 were associated with lower risks of HCC with odds ratio of 0.56 (95\% confidence interval: $0.44-$ 0.70 ) and 0.80 (95\% confidence interval: 0.68-0.94), respectively. Even adjusted with HBV genotype, both SNPs were still associated with HCC development.

Conclusion(s): In Asian HBV carriers, two SNPs, one at HLA-DQ region (rs9275319) and the other at STAT4 region (rs7574865), are shown to be associated with HCC development. 


\section{PS06-008 \\ Metabolic Targeting Inhibits Human Hepatoma Cells in Vitro}

\section{Jung Wha Chung, Eun Sun Jang, Jin-Wook Kim*}

Department of Medicine, SNU Bundang Hospital, Seongnam, Korea

Introduction: Increased glycolysis in the presence of oxygen (Warburg effect) is commonly observed in rapidgrowing human cancer cells. Metabolic targeting is a novel approach to cancer therapy, but the effect is not well known in in human hepatocellular carcinoma. Glucose transporter (GLUT) proteins determine the first rate-limiting step for glucose metabolism and dichloroacetate (DCA), inhibitor of pyruvate dehydrogenase kinase, suppresses glycolytic metabolic pathway. The aims of this study were to determine the effect of suppression of glycolytic pathway by GLUT knockdown or DCA treatment in vitro.

Methods: HepG2, Huh7 cells and non-neoplastic PH5CH8 cells were treated with siRNA against class 1 GLUT family to assess the effect of GLUT knockdown, and also treated with DCA to validate the effect of inhibition of glycolysis on the cell proliferation and apoptosis. Cellular proliferation was assessed by fluorescein diacetate assay, and apoptosis was measured by acridine orange/ethidium bromide staining followed by inverted fluorescent microscopic examination.

Results: Knockdown of GLUT3 with siRNA induced more cell death and apoptosis in HepG2 cells compared to PH5CH8 cells in low glucose concentration of media. DCA suppressed cellular proliferation in Huh7 cells and PH5CH8 cells similarly, whereas treated with DCA induced significantly higher degree of apoptosis in Huh7 cells compared to PH5CH8 cells.

Conclusion(s): Inhibition of glycolysis pathway induces tumor cell death, suggesting their potential role as therapeutic targets.

\section{PS06-009 \\ Hepatocellular Carcinoma in Chronic Hepatitis C Patients According to the Treatment Response to Antiviral Therapy in South Korea \\ Jihye Jihye Jang Young-Joo Jin*, Jin-Woo Lee \\ Internal Medicine, Inha University Hospital, Incheon, Korea}

Introduction: Vaccination against de novo hepatitis $\mathrm{C}$ virus (HCV) infection is currently not available. Therefore, eradication of HCV from the host using antiviral therapy has been performed for the secondary prevention of HCV-associated hepatocellular carcinoma (HCC). However, there has been no sufficient data in HCV-associated HCC incidence according to antiviral therapy in South Korea
Methods: A total of 384 adult patients with treatment naïve for chronic HCV infection between 2003 and 2013 were consecutively enrolled in this study. All patients were positive for anti-HCV antibody and HCV-RNA. Patients were treated with pegylated interferon (PEG-IFN) plus ribavirin, and they were dichotomized as those with sustained virologic response (SVR) and without it.

Results: The median patient's age was 45 years (range, 22-78 years). The most common HCV-genotype were $1 \mathrm{~b}$ (49\%) and $2 \mathrm{a} / \mathrm{c}(42 \%)$. Of the 384 cases, 41 (11\%) patients had liver cirrhosis, that was all compensated state. During the median follow-up period of 32 months after antiviral therapy, HCC developed in $16(4.2 \%)$ patients. The cumulative HCC incidence rates at $1,3,5$, and 7 years were $0 \%, 1 \%, 2 \%$, and $3 \%$, respectively. The cumulative HCC incidence rate at 5 years was tended to be lower in patients with SVR than those without SVR (1.9\% vs. 3.9\%, p = 0.19). However, it was significantly higher in patients with liver cirrhosis than those without it ( $0 \%$ vs. $8.9 \%, \mathrm{p}<0.01$ ).

Conclusion(s): Antiviral therapy for patients with chronic HCV infection using PEG-IFN plus ribavirin appears to be effective at reducing the risk of HCC in patients who achieve SVR, but it seems not to completely eliminate the risk of HCC in infected persons. Cirrhotic background may be more important factor for carcinogenesis in HCV-associated HCC patients.

\section{PS06-010 \\ Opa Interacting Protein 5 (OIP5) Is Involved in Hepatocellular Carcinoma Growth and Metastasis}

Hua Li, Goung-Ran Yu, Mi-Jin Lee, Xue-Ji Han, Dae-Ghon Kim*

Internal Medicine, Chonbuk National University

Medical School, Jeonju, Korea

Introduction: Opa interacting protein 5 (OIP5) was upregulated in some types of human cancers. However, its biological implication and clinical significance have not yet clarified in human cancer, especially in hepatocellular carcinoma (HCC). The aim of study is to examine its biological function and potential as a therapeutic target in HCC development and progression.

Methods: OIP5 expression was strongly detected in early and advanced tumors but barely in normal liver and liver cirrhosis by gene expression profiling and immunohistochemical staining analyses.

Results: OIP5 expression was mainly associated with G2/M phase of the cell cycle progression, which was associated with the regulations of Cdc2 and Cdc25C. The cells with knockdown of OIP5 using target shRNA resulted in reduced hepatic mass forming and metastatic tumor nodules in xenoplant mice model. AKT activation by OIP5 linked to mTORC1 
and GSK-3 $\beta / \beta$-catenin signalings, which are mainly associated with tumor cell proliferation and metastasis, respectively. mTORC1 and GSK-3 $\beta / \beta$-catenin signalings also appeared to affect each other. Epithelial mesenchymal transition (EMT) was modulated by OIP5/AKT and its downstream signaling in HCC.

Conclusion(s): OIP5 may be involved in HCC growth and metastasis through AKT/mTOR and AKT/ $\beta$-catenin signaling pathways, which may be useful therapeutic targets.

\section{PS06-011 \\ The Roles of Ras Isoforms in Liver Cancer \\ Hyuk Moon ${ }^{1}$, Sook In Chung ${ }^{1}$, Hye-Lim Ju', Kwang-Hyub Han², Simon Weonsang Ro ${ }^{2 *}$ \\ ${ }^{1}$ Brain Korea 21 PLUS Project for Medical Science College of Medicine, Yonsei University, Seoul, Korea; ${ }^{2}$ Department of Internal Medicine, Yonsei University College of Medicine, Seoul, Korea}

Introduction: Activation of Ras proteins is a key oncogenic event in human carcinogenesis. Mutations affecting the three prototype Ras oncoproteins, Hras, Nras, and Kras, show a high degree of tumor-type specificity. Kras and Nras are mutated in liver cancer, but Hras mutations are rare. In this study, we determined the liver tumorigenic potentials of the three Ras isoforms.

Methods: Transgenic liver cancer mouse models expressing different Ras isoforms were developed using a hydrodynamic injection method and the Sleeping Beauty Transposon System. Transposon vectors, each encoding an oncogene (HrasG12V, KrasG12V, and NrasG12V) or downregulating a tumor suppressor gene (shp53), were constructed. To induce liver cancer, $40 \mu \mathrm{g}$ of the three plasmids encoding the sleeping beauty transposase and two transposons were diluted in $2.5 \mathrm{ml}$ of $0.9 \%$ saline and injected into the lateral tail veins of 6 -week-old C57BL/6 mice. The mice were observed at 23 days post-hydrodynamic injection or near death.

Results: Co-expression of H-, K-, N-RasG12V and shp53 resulted in massive abdominal enlargement within 4 weeks after injection. Several nodular lesions emerged from the liver parenchyma and occupied most of the liver suRFAce 23 days after injection. The ratio of liver/body weight in the KrasG12V group increased significantly compared to those in the HrasG12V ( $p=0.0005)$ and NrasG12V groups ( $p=0.0181)$. The ratio of the NrasG12V group showed a mild increase compared to that of the HrasG12V group, but this was not significant $(p=0.3819)$. The survival curve of these groups corresponded to the ratio of liver/body weight. All mice were moribund by 36 days.

Conclusion(s): Co-expression of RasG12V and shp53 in the mouse liver promoted rapid hepatocarcinogenesis. In particular, we found that Kras was the most oncogenic among the Ras isoforms in the liver when co-expressed with shp53.

\section{PS06-012 \\ miR-221 and 17-5p Are Overexpressed in Human and Murine Hepatocellular Carcinoma Cells, and Induce Increased Cellular Proliferation and Migration \\ Kyung Joo Cho ${ }^{1}$, Sin Hwa Baek ${ }^{1}$, Sook In Jung ${ }^{1}$, Jun Yong Park'2, Do Young Kim², Kwang Hyub Han², Simon Weon Sang $\mathrm{Ro}^{2 *}$ \\ ${ }^{1}$ Brain Korea 21 PLUS Project for Medical Science College of Medicine, Yonsei University, Seoul, Korea; 2Internal Medicine, Yonsei University College of Medicine, Seoul, Korea}

Introduction: Hepatocellular carcinoma (HCC) is the third-leading cause of cancer-related death worldwide. MicroRNAs (miRNAs) play important roles in gene regulatory networks, and aberrant miRNA expression has been observed in human hepatocarcinogenesis. Molecular pathological studies demonstrated that gene expression alteration is crucial for the development of HCC. The current study aims to identify miRNAs highly expressed in HCC tissues and to investigate their potential roles in tumor development.

Methods: Quantitative RT-PCR (qRT-PCR) assays were performed to compare expression levels of miRNAs between HCC and surrounding non-HCC tissues in humans, and also, in the murine HCC model. Based on the qRT-PCR data, we found several miRNAs highly up-regulated in HCC of both mouse and human. Cell lines stably expressing individual miRNAs were generated using Huh-7 and Hep3B human hepatoma cell lines and the knock-down efficiencies of the expressed miRNAs were tested using a luciferase reporter assay containing antisense sequence of each miRNA. To investigate the functional role played by the miRNAs, cell proliferation and migration assay was performed using the stable cell lines.

Results: miR-221, miR-181b-1, miR-155-5p, miR25, and miR-17-5p exhibited high levels of expression both in murine and human HCC. Stable cell lines expressing miR-221 revealed a higher proliferation potential and those expressing miR17-5p exhibited an increased migration potential.

Conclusion(s): Overexpression of miR-221 and miR17-5p provides an enhanced oncogenic property and thus targeting the miRNAs could be considered as a therapeutic option for HCC patients. 


\section{PS06-013 \\ Differential Tumorigenic Effects by C-Myc Mutants in Liver Cancer}

\author{
Sook In Chung ${ }^{1}$, Hye Lim Ju', Kwang-Hyub Han², \\ Simon W. Ro ${ }^{2 *}$ \\ ${ }^{1}$ Brain Korea 21 PLUS Project for Medical Science \\ College of Medicine, Yonsei University, Seoul, Korea; \\ ${ }^{2}$ Internal Medicine, Yonsei University, Seoul, Korea
}

Introduction: Liver cancer is a major health concern worldwide, ranking third in terms of cancer-related mortality. Nevertheless, its pathogenesis and underlying genetic mechanisms are incompletely understood. The c-Myc gene is overexpressed in almost $50 \%$ of human liver cancers. C-Myc mutations were showed a high degree of tumor-type specificity. In this study, we determined the liver tumorigenic potentials by the c-Myc mutants.

Methods: Transgenic liver cancer mouse models expressing different c-Myc mutant alleles were developed using a hydrodynamic injection method and the Sleeping Beauty Transposon System. Transposon vectors, each encoding an oncogene (c-Myc, c-MycT58A, and c-MycS71F) or down-regulating a tumor suppressor gene (shp53), were constructed. To induce liver cancer, $40 \mu \mathrm{g}$ of the three plasmids encoding the sleeping beauty transposase and two transposons were diluted in $2.5 \mathrm{ml}$ of $0.9 \%$ saline and injected into the lateral tail veins of 6-week-old C57BL/6 mice. Mice were monitored at least twice per week and sacrificed when moribund. Tumor-bearing livers were formalin fixed for hematoxylin-eosin staining.

Results: Hepatocellular carcinomas (HCC) were induced by the stable expression of c-Myc and shp53. Wild type c-Myc was more tumorigenic than c-Myc2T58A or c-Myc2S71F when co-expressed with shp53 (tumor incidences 50\% vs. $20 \%$ ). Interestingly, the mouse survivals did not correspond to the tumor incidences. The c-Myc mutant groups, c-Myc2T58A or c-Myc2S71F died earlier than the c-Myc wild type group $(\mathrm{p}<0.05)$.

Conclusion(s): Co-expression of c-Myc and shp53 in the mouse liver promoted hepatocarcinogenesis. Wild type c-Myc was more tumorigenic than c-Myc2T58A or c-Myc2S71F under the condition that P53 was down-regulated.

\author{
PS06-014 \\ Transgenic Mouse Model Expressing \\ P53R172H, Luciferase, EGFP, and KrasG12D \\ Under a Single Promoter Allows Tumor \\ Growths to Be Monitored Via Live Imaging \\ Hye-Lim Ju' ${ }^{1}$,Hyuk Moon ${ }^{1}$, Sang Hoon Ahn', \\ Kwang-Hyub Han ${ }^{2}$, Weonsang Ro ${ }^{2 *}$ \\ ${ }^{1}$ Yonsei University, Brain Korea 21 PLUS Project for \\ Medical Science College of medicine, Seoul, Korea; \\ ${ }^{2}$ Yonsei University College of Medicine, Department of \\ Internal Medicine, Seoul, Korea
}

Introduction: Genetically engineered mouse (GEM) models for cancer have been popularly used in cancer research due to its similarity to human cancers in various aspects. Live imaging of tumor has been successfully performed in the GEM model via coexpression of GFP or firefly luciferase with a tumor-initiating, genetically engineered gene. However, differential transcriptional and translational regulations between the tumor-initiating gene and the reporter gene can result in an inconsistency between actual tumor sizes and imaging signals. To overcome the limitation, we have developed a transgenic mouse where two types of oncogenes are expressed together with EGFP and firefly luciferase in a single open reading frame via 2A-mediated expression.

Methods: Plasmids were constructed which simultaneously express P53R172H, Luciferase, EGFP, and KRASG12D upon Cremediated DNA excision and used for pronuclear microinjection to generate transgenic founders. After mice were administrated with adenovirus encoding Cre, bioluminescence imaging of abdominal areas was repeatedly performed over months. Liver was harvested at 8 months post adenoviral administration and processed for histo-pathologic analysis.

Results: Repeated bioluminescence imaging of the 2PLEASE mice following adenovirus administration revealed continuous increases in bioluminescent signals over months. A high correlation was found between bioluminescent signals and actual tumor sizes. Tumors induced by P53R172H and KrasG12D revealed the typical morphology of glycogenstoring foci and were diagnosed as hepatocellular adenomas.

Conclusion(s): Simultaneous expression of oncogenes and reporter genes in a single open reading frame was successfully achieved via 2A-mediated ribosome skipping in the transgenic mouse model. The transgenic mouse can be effectively applied to pre-clinical testing of anti-cancer drugs due to its versatility in tracing tumor growths in the liver. 


\section{PS06-015 \\ Resistance to Sorafenib Treatment Is Correlated with Epithelial Mesenchymal Transition in Hepatocellular Carcinoma}

\section{Su Cheol Park*}

Internal Medicine, Korea Institute of Radiological and

Medical Sciences, Seoul, Korea

Introduction: The resistance to conventional treatment is a major obstacle to overcome the hepatocellular carcinoma (HCC). Recently Sorafenib, a multi-kinase inhibitor that targets RAF kinase, VEGFR and PDGFR- $\beta$ tyrosine kinase, has been developed and used for advanced HCC, but the efficacies are not fully satisfied. The exact mechanism for the sorafenib resistance is still unknown. The epithelial mesenchymal transition (EMT) can play an important role in cancer development, invasion and metastasis. The aim of this study was to evaluate the relationship between resistance to sorafenib treatment and EMT.
Methods: Various human HCC cell lines (Huh7, HepG2, Hep3B, SNU387, SNU398 and SNU475) were used in this study. Cell viability and growth inhibition were assessed by MTT. The expressions of EMT-related proteins were evaluated by immunoblot assay. After induction of EMT by Hepatocyte growth factor (HGF), the responses to sorafenib treatment were observed using digital microscope, MTT assay and imunofluorescence staining.

Results: Sorafenib inhibited the growth of HCC cells that expressed E-cadherin, the epithelial marker more significantly than vimentin, the mesenchymal marker. After induction of EMT by HGF in HepG2 and Huh-7 cells, the response of sorafenib was significantly decreased and the cell morphology was changed. In addition, the expression of E-cadherin was decreased and snail expression was increased. Immunofluorescence staining showed disorganized E-cadherin arrangement after HGF treatment.

Conclusion(s): The resistance to sorafenib treatment is correlated with EMT. Thus, EMT can be a prognostic factor for sorafenib treatment and therapeutic target for overcoming resistance to sorafenib in HCC patients. 


\section{Category 7 \\ Recent Progress of Transarterial Treatment}

\author{
PS07-001 \\ The Efficacy and Safety of Hepatic Arterial \\ Infusion Chemotherapy Using System I for \\ Advanced Hepatocellular Carcinoma \\ Akihiro Deguchi ${ }^{1}$, Hiroaki Nagamatsu ${ }^{1 *}$, Rina Tsutsui ${ }^{1}$, \\ Tomoki Shirono ${ }^{1}$, Satoshi Itano ${ }^{2}$, Michio Sata ${ }^{3}$ \\ ${ }^{1}$ Division of Internal Medicine, Yame Republic hospital, \\ Fukuoka, Japan; ${ }^{2}$ Division of Internal Medicine, \\ Itano Clinical hospital, Fukuoka, Japan; ${ }^{3}$ Division \\ of Gastroenterology, Kurume University School of \\ Medicine, Fukuoka, Japan
}

Introduction: Hepatic arterial infusion chemotherapy (HAIC) for unresectable advanced hepatocellular carcinoma (HCC) has sometimes been delivered using interventional percutaneous implanted reservoir-catheter systems (PIRCs). However, several complications are caused by the long-term use of a PIRC for this treatment, including stricture or occlusion of the hepatic artery and peptic ulcer. Because of these complications, continuation of treatment may become impossible. Additionally, unification of the hepatic arterial flow is needed in cases involving a replaced-type hepatic artery. It is widely recognized that unification of the hepatic arterial flow makes it difficult to perform hepatectomy or transcatheter arterial chemoembolization (TACE) post-therapy. At our hospital, we have introduced System I, which is a catheter system that was specifically designed for these cases. System I is implanted into the abdominal aorta from the left humeral artery. In this study, we investigated the efficacy and safety of HAIC using System I for advanced HCC.

Methods: Thirty patients who had HCC (Child-Pugh $A: B=19: 11$; BCLC stage $B: C=6: 24$ ) and received HAIC using System I were enrolled in this study. The patients were classified into 4 groups: (1) 11 patients who discontinued HAIC with PIRC because of complications, (2) 7 patients who had extra-hepatic major arterial feeders, (3) 8 patients who had replaced-type hepatic arteries, (4) 4 patients in whom PIRC implantation was difficult for other reasons. For System I treatment, we puncture the percutaneous implanted port attached to the catheter of System I and insert a micro-catheter through the puncture. The anticancer drug is administrated via this microcatheter, which is inserted into the intra- or extra-hepatic arterial feeder through the side hole of the catheter of System I.

Results: The HAIC regimens were as follows: CDDP only (4 cases), CDDP plus 5-FU (15 cases), epirubicin plus MMC (9 cases), or miriplatin ( 2 cases). The best-response and diseasecontrol rates were $38 \%$ and $82 \%$, respectively. Forty-three percent of cases showed a $>80 \%$ decrease in the level of the tumor marker. A catheter infection developed in 1 case, but serious complications, such as cerebral infarction, were not observed.

Conclusion(s): Our results suggest that HAIC with System I is useful for advanced HCC and may provide an option for the treatment of advanced HCC in cases that are difficult to treat with PIRC.

\section{PS07-002 \\ The Outcomes of Transarterial Infusion of Epirubicin and Cisplatin Combined with Systemic Infusion of 5-Fluorouracil for Unresectable Hepatocellular Carcinoma}

Eun Chung, Seung Kew Yoon*, Sung Won Lee, Jeong Won Jang, Si Hyun Bae, Jong Young Choi

Divison of Hepatology, Department of Internal Medicine, College of Medicine, The Catholic University of Korea, Seoul, Korea

Introduction: As a novel therapeutic strategy for unresectable hepatocellular carcinoma (HCC), we performed and analyzed the effects and toxicity of transarterial infusion of epirubicin and cisplatin combined with systemic infusion of 5-fluorouracil (TAC-ECF).

Methods: A total of 130 intermediate to advanced stage HCC patients who received at least one session of TAC-ECF at Seoul St Mary's hospital were analyzed. The mixture of epirubicin, cisplatin and lipiodol was administered via hepatic artery followed by systemic 5-fluorouracil after the TAC. The best response within 6 months after the 1st TAC-ECF session was defined as the tumor response. We analyzed the tumor response, disease progression free survival time, overall survival time and the predictive factors for each survival times.

Results: During the median follow up period of 45 months, patients received median 3 sessions of TAC-ECF. Complete remission was obtained in 28 (22\%), partial response in 27 (21\%), stable disease in 51 (39\%), and progressive disease in $22(17 \%)$ patients respectively. Disease progression free time from the 1st TAC ECF session was median 7 months. Overall survival time was median 51.4 months from the diagnosis of HCC and 25.4 months from the 1st TAC ECF session. Cumulative survival rates at 6,12, 18 and 24 months were $90 \%, 73 \%, 66 \%$ and $57 \%$ respectively. No serious adverse effects developed after the treatment.

Conclusion(s): TAC-ECF treatment was tolerable and showed higher tumor response and overall survival rates 
compared to conventional transarterial chemoembolizations (TACEs). Therefore, TAC-ECF may be an effective treatment option for unresectable HCCs.

\section{PS07-007 \\ Retreatment with Transarterial Chemoembolization in Patients with Hepatocellular Carcinoma: Verification of Art Score in a Single Institution}

\section{I-Lun Shih ${ }^{1}$, Bang-Bin Chen ${ }^{1}$, Chih-Horng Wu ${ }^{1}$, Chien-Hung Chen ${ }^{2}$, Kao-Lang Liu ${ }^{1}$, Po-Chin Liang ${ }^{1}$, Tiffany Ting-Fang Shih ${ }^{1 *}$ \\ ${ }^{1}$ Department of Medical Imaging, National Taiwan University Hospital, Taipei, Taiwan; ${ }^{2}$ Department of Internal Medicine, National Taiwan University Hospital Taipei, Taiwan}

Introduction: Sieghart et al. had developed a point score (ART score: Assessment for Retreatment with Transarterial chemoembolization (TACE)) to guide the decision for retreatment with TACE in patients with hepatocellular carcinoma (HCC). This score could help identifying patients with a dismal prognosis who may not benefit from further TACE. The objective of this study is to apply the ART score to our patient referral population.

Methods: We performed a retrospective review of the patients who received TACE as an initial therapy for HCC in our hospital from January 2009 to December 2009, who also had a second session of TACE within 180 days after the first TACE. The laboratory data and image findings before the two TACE sessions were reviewed. The radiologic tumor response was evaluated using modified response evaluation criteria in solid tumors (mRECIST). The ART score is determined by the

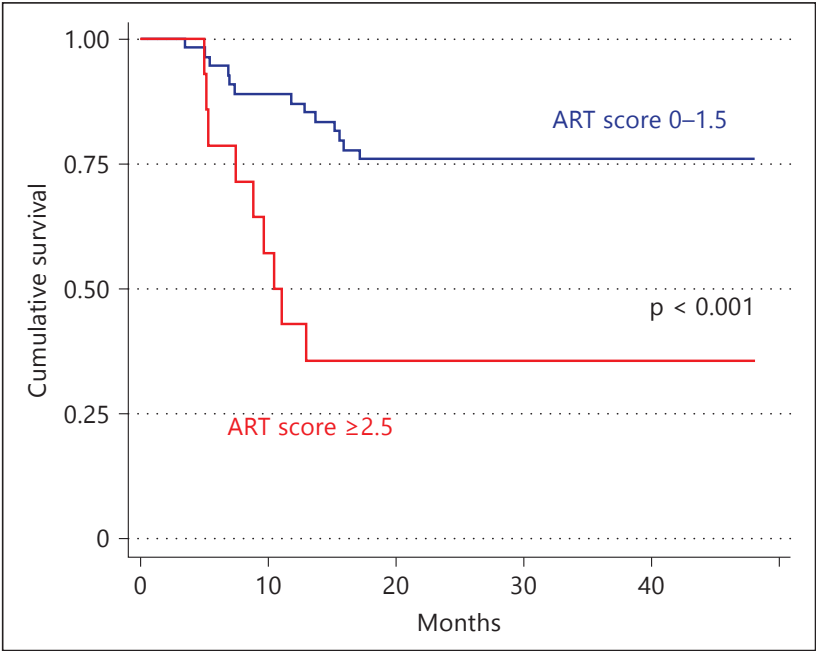

Fig. 1. Prognostic significance of the ART score in different patient subgroups (for abstract PS07-007). increase of aspartate aminotransferase (AST) by $>25 \%$, the increase of Child-Pugh score of 1 or $\geq 2$ points, and the absence of radiologic tumor response. We analyzed the result of ART score and its impact on overall survival.

Results: Total 68 patients were included in the study population (46 men, 22 women; mean age $62 \pm 13$, range 21-85), with a follow up period of $31.7 \pm 15.8$ months. Most of the patients $(87 \%)$ had a viral etiology, which is the main difference from the study population of Sieghart et al. The ART score differentiated two patient groups (0-1.5 points, 54 patients $(79 \%)$; $\geq 2.5$ points, 14 patients $(21 \%)$ ) with significant difference in prognosis (median overall survival: 40.8 versus 10.9 months; $\mathrm{P}<0.001$ ).

Conclusion(s): Our study validated the application of ART score in a patient group in East Asia with mainly viral etiology.

\section{PS07-008 \\ Inter-Observer Variability of EASL Criteria and Modified Recist for Hepatocellular Carcinoma Treated with Chemoembolization}

Beom Kyung Kim ${ }^{1}$, Wonseok Kang ${ }^{1}$, Mi Na Kim¹, Kyu Sik Jung ${ }^{1}$, Seung Up Kim ${ }^{1 *}$, Kyung Ah Kim², Myeong-Jin Kim³, Jun Yong Park ${ }^{1}$, Do Young Kim ${ }^{1}$, Sang Hoon Ahn ${ }^{1}$, Kwang-Hyub Han ${ }^{1}$, Mi-Suk Park ${ }^{3}$

${ }^{1}$ Internal Medicine, Yonsei University College of Medicine, Korea; ${ }^{2}$ Radiology, The Catholic University of Korea, Korea; ${ }^{3}$ Radiology, Yonsei University College of Medicine, Korea

Introduction: European Association for the Study of the Liver (EASL) criteria and modified Response Evaluation Criteria in Solid Tumors (mRECIST) are standard methods for response evaluation in hepatocellular carcinoma (HCC). We evaluated inter-observer variability assessed by two criteria after chemoembolization.

Methods: For 133 treatment-naïve patients with HCC undergoing chemoembolization, two radiologists independently measured sums of bi-dimensional/uni-dimensional diameters (SBDs/ SUDs) at baseline using EASL criteria/ mRECIST, respectively and their changes on first follow-up for up to 5 target lesions. Concordance correlation coefficients (CCC) and Bland-Altman plots were used for inter-observer agreement. Regarding treatment response (complete response, partial response, stable disease and progression), kappa-statistics were used.

Results: CCCs for SBDs and SUDs at baseline between two observers were 0.992 and 0.988 , respectively. However, those for their changes on follow-up became lower; 0.865 and 0.877 , respectively. Similarly, mean differences in SBDs and SUDs at baseline were small; $-0.455 \mathrm{~cm}^{2}$ (95\% confidence interval [CI] -12.631 11.721) and $0.079 \mathrm{~cm}$ (95\% CI -1.546 1.704), respectively. However, mean differences in changes $(\%)$ in 
SBDs and SUDs became larger; $-9.715 \% \quad(95 \%$ CI -43.486 24.056) and -9.320\% (95\% CI -38.844 20.204), respectively. Regarding tumor numbers, kappa-value between two observers was 0.942. Regarding treatment response, those were all 0.941 by both criteria. When only up to two target lesions assessed, those were all 1.000 by both criteria.

Conclusion(s): Despite potential for misclassification of treatment response with some variability of changes (\%) in SBDs or SUDs on follow-up, response evaluation using both criteria showed excellent inter-observer agreements, especially when up to two target lesions assessed.

\section{PS07-009 \\ Transarterial Chemoembolization (TACE) with Polyvinyl Alcohol and ADM+Lipiodol Mixture \\ Myung Gyu Song ${ }^{1}$, Tae-Seok Seo ${ }^{1 *}$, Yun Hwan Kim², Sung Bum $\mathrm{Cho}^{2}$, Hwan Hoon Chung ${ }^{3}$, Seung Hwa Lee ${ }^{3}$ \\ ${ }^{1}$ Radiology, Korea University Guro Hospital, Seoul, Korea; ${ }^{2}$ Radiology, Korea University Anam Hospital, Seoul, Korea; ${ }^{3}$ Radiology, Korea University Ansan Hospital, Seoul, Korea}

Introduction: To present our experience of polyvinyl alcohol as embolic material used in large hepatic carcinoma with extensive intratumoral collateral vessels and arteriopotal shunt during transarterial chemoembolization

Methods: We will present 3 cases of patients who presented to our institution with huge HCC and extensive intratumoral collateral vessels which were managed using TACE with PVA. We often used PVA for HCC with intratumoral collateral vessels and arteriopotal shunt during TACE. We experienced extensive tumoral necrosis after TACE with PVA treatment for tumor. CT on follow up showed extensive necrosis with multiple air densities in the mass.

Conclusion(s): These cases will give a better understanding how we use PVA and effect of PVA for intratumoral collateral vessels.

\section{PS07-010 \\ A Comparative Study for Survival Benefits and Safety of Hepatic Arterial Infusion Chemotherapy for Advanced Hepatocellular Carcinoma in Alcoholics versus Non- Alcoholics \\ Myung Jin $\mathrm{Oh}^{*}$ \\ Department of Internal Medicine, CHA Gumi Medical Center CHA University School of Medicine, Gumi, Korea}

Introduction: Sorafenib, a multikinase inhibitor, has been recommended as a standard of care for advanced hepatocellular carcinoma (HCC). However, sorafenib is limit to use in many Asian countries including Korea. Hepatic arterial infusion chemotherapy (HAIC) can be an alternative option for intractable advanced HCC. In Korea, excessive alcohol intake is one of major causes of HCC. Thus, the aim of this study was to compare survival benefits and safety of HAIC for advanced HCC between the alcoholic patients and the nonalcoholic patients.

Methods: From January 2009 to December 2011, a total of 51 patients who received only HAIC with 5 -fluorouracil (750 mg/m2 on days 1-4) and cisplatin (25 mg/m2 on days 1-4) for advanced HCC over one cycle were enrolled. The selected subjects were divided into the alcoholics group ( $\mathrm{n}=$ $21)$ and the non-alcoholics group $(n=30)$. Alcoholics was defined as a regular average consumption of $>20 \mathrm{~g} /$ day for women, $>40 \mathrm{~g} /$ day for men. After HAIC for advanced HCC, the overall survival (OS), progression-free survival (PFS), and adverse events in the alcoholics group and the non-alcoholics group were investigated retrospectively.

Results: No significant difference was observed in baseline characteristics between the alcoholics group and the non-alcoholics group. Median OS and PFS in the two groups were 5.1 months (95\% confidence interval [CI]: 2.0-8.2) vs. 5.4 months (95\% CI: 2.0-8.7) and 4.4 months (95\% CI: 3.7-5.2) vs. 4.6 months (95\% CI: 2.5-6.7), respectively. Median OS and PFS were not statistically significant between the two groups ( $p=0.310, p=0.375$ ). In addition, the serious adverse events $\geq$ grade 3 arose from 21 patients $(100 \%)$ in the alcoholics group and 26 patients (83.9\%) in the non-alcoholics, respectively. Occurrence of serious adverse events did not differ in the two groups ( $\mathrm{p}=0.143)$.

Conclusion(s): Our study demonstrated that survival benefits and safety of HAIC for advanced HCC of alcoholic patients were comparable to those of non-alcoholic patients. 


\section{PS07-011 \\ Intraarterial Chemotherapy with Doxorubicin and Cisplatin Is Effective for Advanced Hepatocellular Cell Carcinoma}

Yen-Yang Chen ${ }^{1}$, Shau-Hsuan $L^{1}{ }^{1}$, Yu-Fan Cheng ${ }^{2}$, Chih-Chi Wang ${ }^{3}$, Tai-Jan Chiu', Sung-Nan Pei ${ }^{1}$, Chien-Ting Liu', Tai-Lin Huang ${ }^{1}$, Chen-Hua Huang ${ }^{1}$, Yu-Li Su', Yen-Hao Chen ${ }^{1}$, Sheng-Nan Lư Kun-Ming $\operatorname{Rau}^{1 *}$

${ }^{1}$ Division of Hematology-Oncology, Department of Internal Medicine, Kaohsiung Chang Gung Memorial Hospital of the C.G.M.F., Kaohsiung, Taiwan; ${ }^{2}$ Department of Diagnostic Radiology, Kaohsiung Chang Gung Memorial Hospital of the C.G.M.F., Kaohsiung Chang Gung Memorial Hospital of the C.G.M.F., Kaohsiung, Taiwan; ${ }^{3}$ Department of Surgery, Kaohsiung Chang Gung Memorial Hospital of the C.G.M.F., Kaohsiung Chang Gung Memorial Hospital of the C.G.M.F., Kaohsiung, Taiwan; ${ }^{4}$ Division of HepatoGastroenterology, Department of Internal Medicine, Kaohsiung Chang Gung Memorial Hospital of the C.G.M.F., Kaohsiung Chang Gung Memorial Hospital of the C.G.M.F., Kaohsiung, Taiwan

Introduction: Advanced hepatocellular carcinoma (HCC) remains a fatal disease even in the era of targeted therapies. Intraarterial chemotherapy (IACT) can provide therapeutic benefits for patients with locally advanced HCC who are not eligible for local therapies or are refractory to targeted therapies. The aim of this retrospective study was to analyze the effect of IACT with cisplatin and doxorubicin on advanced HCC.

Methods: Patients with advanced HCC who were not eligiblefor local therapies or were refractory to sorafenib received doxorubicin $\left(50 \mathrm{mg} / \mathrm{m}^{2}\right)$ and cisplatin $\left(50 \mathrm{mg} / \mathrm{m}^{2}\right)$ infusions into the liver via the transhepatic artery every 4 weeks.
Results: Between January 2005 and December 2011, a total of 50 patients with advanced HCC received this treatment regimen. The overall response rate (ORR) was $22 \%$ in all treated patients. In patients who received at least 2 cycles of IACT, the ORR was $36.7 \%$, and the disease control rate was $70 \%$. Survival rate differed significantly between patients who received only one cycle of IACT (group I) and those who received several cycles (group II). The median progression-free survival was 1.3 months and 5.8 months in groups I and II, respectively $(p<0.0001)$. The median overall survival was 8.3 months for all patients, and was 3.1 months and12.0 months in groups I and II, respectively ( $\mathrm{p}<$ $0.0001)$. The most common toxicity was alopecia. Four patients developed grade 3 or 4 leukopenia. Worsening of liver function, nausea and vomiting were uncommon side effects.

Conclusion(s): This study demonstrated clinical efficacy and tolerable side effects of repeated IACT with doxorubicin and cisplatin in advanced HCC. Our regimen can be an alternative choice for patients with adequate liver function who do not want to receive continuous infusion of IACT.

\section{PS07-012 \\ Survival Rates and Prognostic Factors for Unresectable Hepatocellular Carcinoma after Transcatheter Arterial Chemoembolization}

\section{Amna Butt ${ }^{1 *}$, Saeed Hamid ${ }^{1}$, Nazish Butt ${ }^{1}$, Tanveer-ul Haq², Sajjad Ali', Ayesha Majeed ${ }^{1}$, Wasim Jafri ${ }^{1}$ \\ ${ }^{1}$ Medicine, Aga Khan University Hospital, Karachi, Pakistan; ${ }^{2}$ Radiology, Aga Khan University Hospital, Karachi, Pakistan}

Introduction: Transcatheter arterial chemoembolization (TACE) is recommended as palliative treatment for unresectable Hepatocellular carcinoma (HCC). No data is available from Pakistan in this regard.

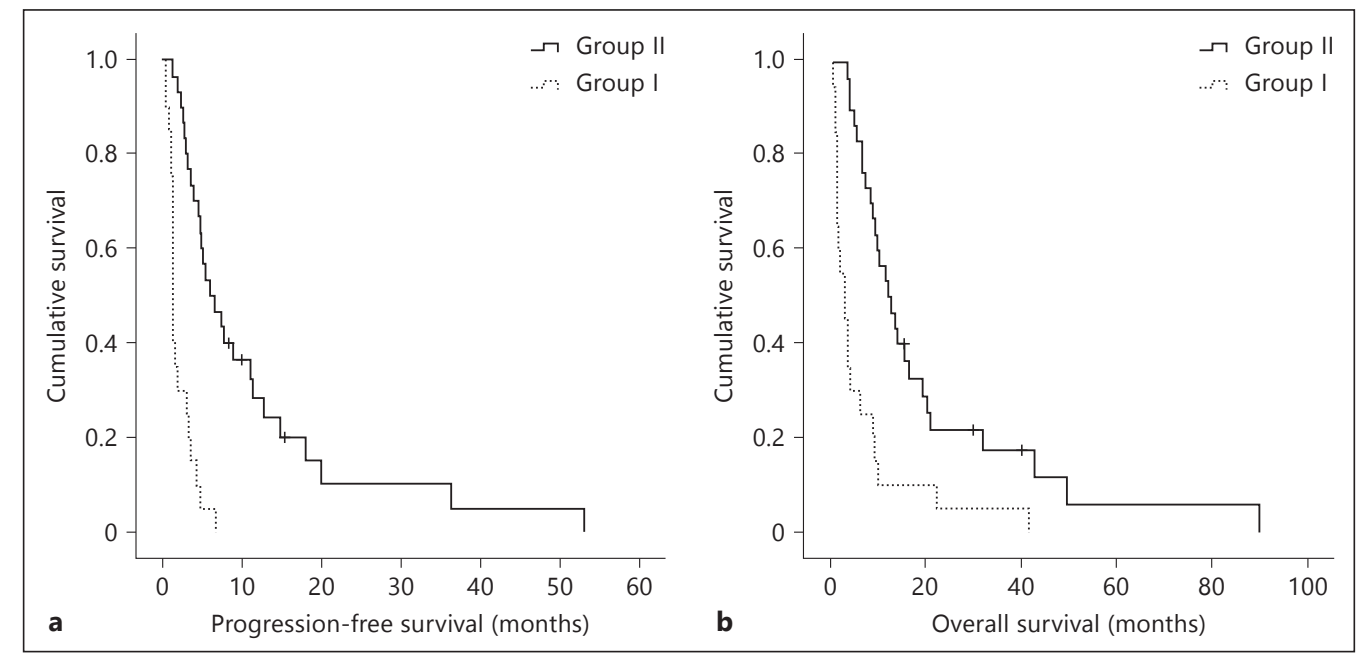

Fig. 1. (for abstract PS07-011). 
The 5th Asia-Pacific Primary Liver Cancer Expert Meeting (APPLE 2014)

Aims: To estimate the survival of the patients with unresectable HCC treated with TACE and to analyze the prognostic factors affecting survival.

Methods: All patients diagnosed with unresectable HCC who underwent for TACE during 2000-2012 at The Aga Khan University Hospital, Karachi, Pakistan were reviewed. Information was collected regarding demographic characteristics, baseline laboratory parameters, tumor characteristics and staging, response to TACE and survival. Survival at 1, 3 and 5 years and the predictors for survival were estimated.

Results: TACE was performed in 503 patients with HCC. Mean age was $56.9 \pm 10.47$ years and $77.7 \%$ were males. Hepatitis C was the most prevalent (70\%) etiological factor. Mean Childs and MELD score was 6.9 \pm 1.5 and $19.34 \pm 4.70$ respectively. Median AFP was 47.5 (range 1.20-300000) IU/ml. The mean tumor size was $6.1 \pm 3.31 \mathrm{~cm}$. A total of $46.6 \%, 22.0 \%$, $22.6 \%$ and $8.8 \%$ had solitary, paucifocal, multifocal and infiltrative/massive HCC respectively. Majority of patients $(67.2 \%)$ had one session of TACE while two sessions were done in $21.9 \%$, three sessions in $5.8 \%$, four sessions in $2.2 \%$ and five sessions were done in $2.1 \%$ patients. The follow-up duration ranged 1-120 months. The overall median survival was 38.000 months (95\% CI 29.04-47.0). Moreover, the cumulative 1-year, 3-year, and 5-year survival rates were $82 \%, 38 \%$, and $20 \%$, respectively.

Age $>65$ years (Hazard ratio 2.50; 5\% CI 1.40-4.47; p 0.002), Child's class B (Hazard ratio 2.54; 5\% CI 1.48-4.38; p 0.001 ), advanced HCC (Hazard ratio 2.13; 95\% CI 1.29-3.52; p 0.003) and incomplete tumor response (Hazard ratio 2.12; CI 95\% 1.28-3.05; p-value 0.02) were the factors associated with a poor survival.

Conclusion(s): TACE was useful and well tolerated palliative therapy for unresectable HCC. Age $>65$ years, Child's class B, advanced HCC and incomplete tumor response were the factors associated with a poor survival.

\section{PS07-013 \\ Outcome of Endoscopic Biliary Drainage in Advanced Hepatocellular Carcinoma with Obstructive Jaundice}

\author{
Hyun Young Woo Jeong Heo*, Dong Uk Kim, \\ Won Lim, Ki Tae Yoon, Young Mi Hong \\ Department of Internal Medicine, Pusan National \\ University Hospital, Busan, Korea
}

Introduction: Advanced hepatocellular carcinoma (HCC) with jaundice has an extremely poor prognosis. However, in case of obstructive jaundice, biliary drainage can resolve obstructive jaundice. We evaluated the role of endoscopic biliary drainage in patients with advanced HCC with obstructive jaundice.

Methods: From 2010 to 2013, total 26 who received endoscopic biliary drainage for obstructive jaundice due to advanced HCC was retrospectively reviewed. Success rate of biliary drainage, jaundice resolution (complete resolution defined as less than $2 \mathrm{mg} / \mathrm{dl}$, partial resolution defined as decreased but $>2 \mathrm{mg} / \mathrm{dl}$ ), survival rate was assessed.

Results: The median age was 61 years (range 37-78 years) and $88.5 \%$ (23/26) was male. Hepatitis B was $69.2 \%$ $(18 / 26)$, hepatitis $\mathrm{C}$ was $15.4 \%(4 / 26)$ and unknown was $15.4 \%(4 / 26)$. Child-Pugh class was B in $84.6 \%(22 / 26)$, C in $15.4 \%(4 / 26)$. Portal vein invasion was observed in $69.2 \%$ $(18 / 26)$. BCLC stage was B in $11.5 \%(3 / 26)$ and C in $88.5 \%$ $(19 / 26)$. Okuda stage is stage I in $3.8 \%(1 / 26)$, II in $61.5 \%$ $(16 / 26)$ and III in $34.6 \%$ (9/26). Intrahepatic bile duct was dilated in $69.2 \%(18 / 26)$ and location was right in $61.5 \%$ $(16 / 26)$. After drainage, jaundice was resolved partially in 8 $(30.8 \%)$ and completely in $9(34.6 \%)$. The median duration to resolution was 19 days (range 2-96 days). However, jaundice was reaggravated in $70.6 \%$ (12/17) after median 47.5 days (range 5-399 days). In the other 9, jaundice was not improved or worsened after drainage. The 90 days, 1 year survival rate was $36.8 \%, 13.8 \%$, respectively. Median survival was 89 days (95\% CI 39.49-138.51 days). In multivariate analysis, response to biliary drainage was significantly associated with survival $(\mathrm{P}=0.029)$.

Conclusion(s): Through endoscopic biliary drainage, obstructive jaundice was improved in $65.4 \%$ with advanced HCC and survival can be prolonged in these patients. Biliary drainage can be appropriate palliative treatment in advanced HCC patients. 


\section{Category 8 \\ Unresolved Issues for Advanced HCC}

\author{
PS08-001 \\ Impact of Hepatitis C Virus Etiology on \\ the Efficacy of Sorafenib in Advanced \\ Hepatocellular Carcinoma: A Meta-Analysis \\ Yu-Yun Shao ${ }^{1}$, Wen-Yi Shau', Soa-Yu Chan', \\ Li-Chun Lu', Chih-Hung Hsu', Ann-Lii Cheng ${ }^{1 *}$ \\ ${ }^{1}$ Department of Oncology, National Taiwan University \\ Hospital, Taipei, Taiwan; ${ }^{2}$ Pfizer Inc., New Taipei, Taiwan
}

Introduction: Previous studies suggested that hepatocellular carcinoma (HCC) of hepatitis C virus (HCV) etiology may benefit more from sorafenib. We planned to clarify this issue by conducting a meta-analysis on data of phase III clinical trials.

Methods: PubMed and Cochrane library were searched for phase III clinical trials that compared sorafenib with other treatments as the first-line therapy for advanced HCC. We retrieved data from the published articles and then calculated synthesized hazard ratios (HRs) for patients of different subgroups.
Results: Four phase III clinical trials comparing sorafenib with other treatments were included. Among the 3057 patients in total, the HCC etiology was HCV in 653 patients, hepatitis B virus (HBV) in 1315 patients, and alcohol consumption in 521 patients. When sorafenib was compared with other treatments, the synthesized HR of all-cause deaths in the entire population was 0.81 , favoring sorafenib. The synthesized HR of HCV (+) patients was 0.65 (95\% confidence interval [CI], 0.53-0.80), which was significantly lower than that of $\mathrm{HCV}(-)$ patients $(0.87,95 \% \mathrm{CI} 0.79-0.96, \mathrm{P}=0.013)$ or that of HBV $(+)$ patients $(0.92,95 \%$ CI $0.82-1.05, \mathrm{P}=0.004)$. By contrast, the HRs of patients from different geographic regions (Asia vs. non-Asia $=0.85$ vs. 0.75 ) or with different Eastern Cooperative Oncology Group performance statuses (0 vs. 1 or $2=0.85$ vs. 0.81 ) were not significantly different.

Conclusion(s): As the first-line therapy for advanced HCC, sorafenib provided more survival benefits to HCV (+) patients than to HCV (-) patients. This finding cannot be explained by different geographic regions or performance status of the patients.

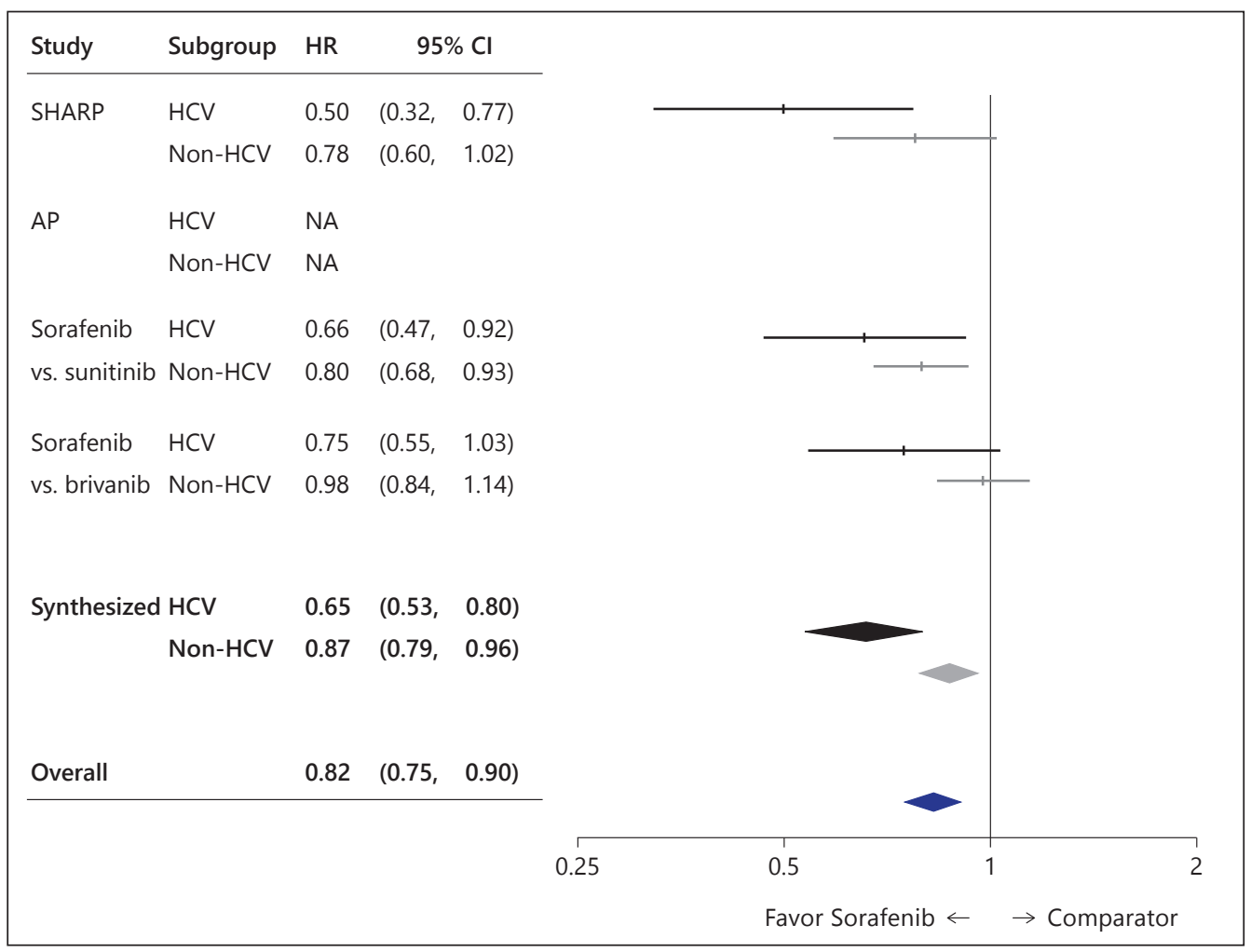

Fig. 1. (for abstract PS08-001). 


\section{PS08-003 \\ Risk Factors of Radiotherapy Induced Liver Disease (RILD) in Patients with Advanced Hepatocellular Carcinoma

\author{
Hung-Chuen Chang Yu-Min Lin*, Chao-Sheng Liao, \\ Kuo-Ching Yang \\ Internal Medicine, Gastroenterology, Taipei, Taiwan
}

Introduction: The use of radiotherapy for hepatocellular carcinoma (HCC) is rapidly increasing. The aim of this study was to understand the survival outcome and risk factors of radiotherapy induced liver disease (RILD) in the patients with advanced HCC.

Methods: We retrospectively analyzed the patients of advanced HCC (BCLC stage C) treated with Intensity Modulation Radiation Therapy (IMRT) or Tomothearpy between January 2008 and December 2011. Classic presentation of RILD was defined as anicteric hepatomegaly, ascites and isolated elevated alkaline phosphatase occurred within 4 months after completion of radiotherapy. Non-classic presentation of RILD was defined as jaundice and ALT more than 5 times of upper normal limit occurred within 3 months after completion of radiotherapy. The variables including age, gender, tumor characters, liver function, treatment modalities and survival outcome are compared between patients with and without RILD. Logistic regression analysis, Kaplan-Meier analysis and log-rank test are used to examine the statistic significance.

Results: A total of 61 patients were identified and 8 were excluded because of inadequate clinical information. Finally, 53 patients [43 male (81.1\%) and 10 female (18.9\%)] were enrolled for subsequent analysis. The mean age of the patients was $57.2 \pm 13.3$ years. Among 53 individuals, 22 (41.5\%) developed RILD within 4 months after completion of radiotherapy. The progression of jaundice and ascites were the most common presentations of RILD. The median survival time in patients with and without RILD were 4.5 and 17.8 months respectively $(\mathrm{p}<0.01)$ (Figure). Logistic regression analysis revealed treatment modality (IMRT) and baseline liver function (Child-Pugh score $>6$ ) were significantly associated with the increased risk of RILD.

Conclusion(s): RILD is common in patients of HCC after radiotherapy and is associated with a worse survival outcome. Selection of the modality and evaluation of liver function before radiotherapy are important to avoid RILD.

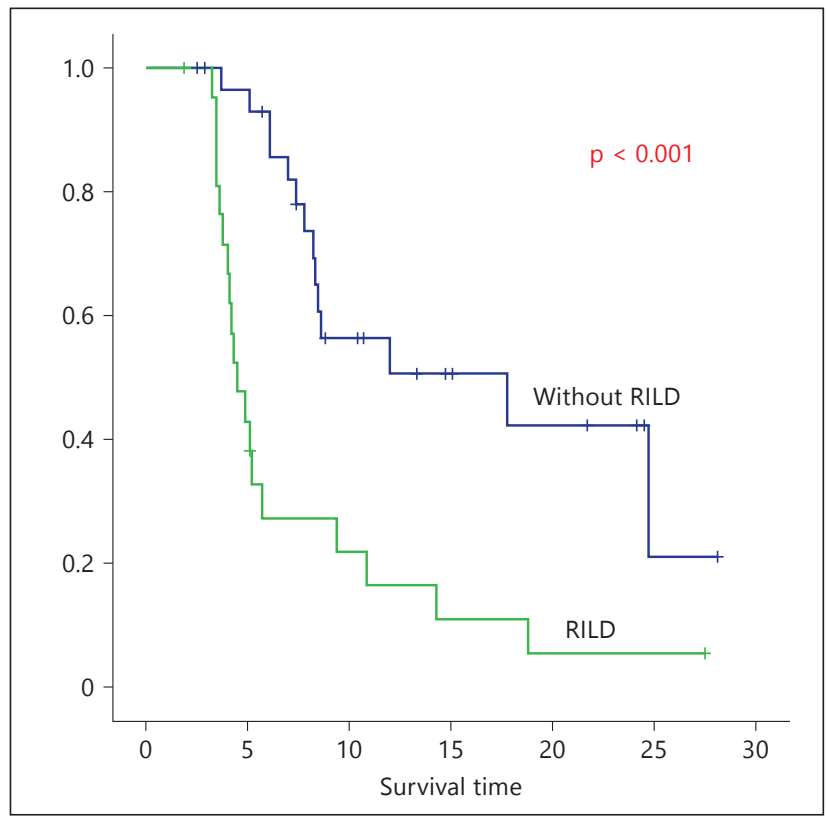

Fig. 1. Survival of HCC with/without RILD (for abstract PS08003).

\section{PS08-007 \\ The Signal Change of the Normal Liver around Hepatocellular Carcinoma after Three Dimensional Conformal Radiotherapy \\ Won Sup Yoon ${ }^{1 *}$, Nam Kwon Lee ${ }^{2}$, Jung Ae Lee ${ }^{2}$, Dae Sik Yang ${ }^{2}$, Chul Yong Kim², Sang Jun Suh', Hyung Joon Yim³, Suk Keu Yeom ${ }^{4}$, Seung Hwa Lee, Hwan Hoon Chung ${ }^{4}$, Sang Hoon $\mathrm{Cha}^{4}$ \\ ${ }^{1}$ Radiation Oncology, Korea University, Ansan, Korea; \\ ${ }^{2}$ Radiation Oncology, Korea University, Seoul, Korea; \\ ${ }^{3}$ Internal Medicine, Korea University, Ansan, Korea; \\ ${ }^{4}$ Radiology, Korea University, Ansan, Korea}

Introduction: Radiotherapy is indicated for advanced hepatocelluar carcinoma with refractory or insufficient response to transarterial chemoembolization. Our study observed the early and late MRI-signal change of normal liver after radiotherapy according to the levels of radiation dose.

Methods: From September 2009 to June 2013, 21 patients were retrospectively evaluated. Radiotherapy consisted of total 40 Gy by 16 fractions or 54 Gy by 27 fractions. All the patients with Child-Pugh grade A or B received 3D conformal radiotherapy and periodic MRI examination was done. Early and late phase were defined as the period from 2 to 4 months and from 10 to 14 months after a completion of radiotherapy, respectively. The images of simulation CT and follow-up MRI, and the information of dose distribution were fused using deformable registration software (MIM version 6.1). To observe the signal changes of MRI, the five 
sets of T1-, T2- and diffusion-weight images, and dynamic contrast images of arterial and biliary phase were used. On every MRI, the five virtual spheres with a diameter between 10 and $15 \mathrm{~mm}$ were drawn according to the isodose levels of total irrradiated dose (over $90 \%$ (very high), between $90 \%$ and $60 \%$ (high), between $60 \%$ and $30 \%$ (mid), between $30 \%$ and $5 \%$ (low), within 5\% (weak)) and mean signal intensity was measured. A ratio between the signal intensity of a weak area and other areas was calculated. An ANOVA test was done to compare signal differences between each area of different dose distribution.

Results: Median age was 57 years (range, 41-74 years) and three were female. Total 19 and 11 cases of early and late phase MRI were analyzed, respectively. The median period between the completion of radiotherapy and follow-up MRI was 3.4 months for early phase and 11 months for late phase. Patients with Child-Pugh grade A were 71.4\% (15/21), 73.7\% $(14 / 19)$ and $77 \%(7 / 11)$ at the time of start of radiotherapy, early MRI and late MRI, respectively. On early MRI, the very high area of biliary phase and T2-weight images was stratified with others and weak area showing the strong hypo-signal $(p<0.001)$ and the mild hyper-signal $(p=0.048)$, respectively. On late MRI, the very high area of biliary phase and T2-weight images was stratified with mid, low and weak areas showing the significant hypo-signal $(\mathrm{p}=0.005)$ and hyper signal $(\mathrm{p}<$ $0.001)$, respectively. The similar outcomes were observed in the cohort with Child-Pugh grade A of both early and late MRI.

Conclusion(s): The MRI images of biliary phase well reflected the radiation field over $90 \%$ of prescribed radical irradiation dose.

\section{PS08-008 \\ Sorafenib Decreased Side Population Targeting JNK Signaling \\ Jong Bin Kim ${ }^{1}$, Seo-Young Park², Min Hong Lee ${ }^{1}$, Seulki Lee ${ }^{1}$, Jeong-Hoon Lee ${ }^{1}$, Su Jong Yu ${ }^{1}$, Hyo-Suk Lee ${ }^{1}$, Jung-Hwan Yoon ${ }^{1}$, Yoon Jun Kim ${ }^{1 *}$ \\ ${ }^{1}$ Department of Internal Medicine, Seoul National University College of Medicine, Liver Research Institute, Seoul, Korea; ${ }^{2}$ Seoul National University Hospital, Biomedical Research Institute, Seoul, Korea}

Introduction: Liver cancer is one of the most fatal cancers in Korea. Many anti-cancer drugs have been used to treat liver cancer, however, failed in liver cancer therapy. Sorafenib, multiple kinase inhibitor, is used to treat liver cancer. We sought to evaluate the effect of sorafenib as an anti-cancer drug targeting cancer stem cells through analysis of side population cells and signaling related in drug-resistance in hepatocarcinoma cells.

Methods: To study the effect of sorfenib, huh7 and huh-bat cells were treated by sorfenib, 5-FU and sorafenib plus 5-FU. These cells were examined growth rate, the SP fraction, expression of cancer stem cells marker, and expression of JNK signaling molecules.

Results: Both sorafenib and 5-FU decreased growth rate. However, there were differences in SP cell, expression of cancer stem cell marker, and expression of JNK signaling molecules. 5-FU induced the increase in SP cell and upregulation of cancer stem cell marker and JNK signaling molecules. On the other hand, sorafenib induced the decrease in SP cells and blocked the upregulation of cancer stem cell marker and JNK signaling by 5 -FU. We found that 5 -FU induced the decrease in growth rate, the increase in SP cells, and upregulation of cancer stem cell marker and JNK signaling. Whereas, sorafenib induced the decrease in both growth rate and SP cells, and blocked the upregulation of cancer stem cell marker and JNK signaling by 5-FU.

Conclusion(s): These results indicated that the effect of sorfenib as an anti-cancer drug for hepatocarcinoma cells could result in targeting cancer stem cells and signaling related in drug-resistance.

\section{PS08-010 \\ Alpha-Fetoprotein and Des-Gamma-Carboxy Prothrombin Responses Mid-Course through Hepatic Arterial Infusion Chemotherapy for Advanced Hepatocellular Carcinoma as Predictors of Clinical Outcome

\author{
Isseki Saeki ${ }^{*}$, Takahiro Yamasaki ${ }^{2}$, Shuji Terai ${ }^{1}$, \\ Isao Sakaida ${ }^{1}$ \\ ${ }^{1}$ Department of Gastroenterology and Hepatology, \\ Yamguchi University Graduate School of Medicine, \\ Ube, Japan; ${ }^{2}$ Department of Oncology and Laboratory \\ Medicine, Yamguchi University Graduate School of \\ Medicine, Ube, Japan
}

Introduction: The prognosis of advanced hepatocellular carcinoma (HCC) remains poor. Hepatic arterial infusion chemotherapy (HAIC) is a treatment for patients with advanced HCC. We investigated whether alpha-fetoprotein (AFP) and des-gamma-carboxy prothrombin (DCP) responses at mid-course of HAIC treatment were predictors of response and survival in advanced HCC patients.

Methods: We retrospectively enrolled 90 patients receiving HAIC who had elevated baseline AFP and/or DCP levels and analyzed various parameters for their possible use as predictors of response and survival. AFP and DCP responses were assessed at the end of a half course of HAIC ( 2 weeks); a response was defined as a reduction of $>20 \%$ from baseline.

Results: The radiological response rate was $51.9 \%$ in AFP responders and $21.4 \%$ in AFP non-responders $(\mathrm{P}=$ $0.0089)$. The radiological response rate was $55.0 \%$ in DCP responders and $14.3 \%$ in DCP non-responders ( $\mathrm{P}=0.0001)$. AFP and DCP responders had significantly longer survivals than non-responders (AFP, $\mathrm{P}=0.0020$; DCP, $\mathrm{P}=0.0047$ ). 
Multivariate analysis identified that DCP responder [odds ratio 6.72, 95\% confidence interval (CI) 1.92-27.94; $\mathrm{P}=$ $0.0024]$ independently predicts radiological response. ChildPugh class A (hazard ratio [HR] 2.06, 95\% CI 1.16-3.65; P = 0.0140), AFP responders (HR 2.24, 95\% CI 1.24-4.16; $\mathrm{P}=$ 0.0071 ), and DCP responders (HR 2.05, 95\% CI 1.10-3.88; $\mathrm{P}=$ 0.0223 ) were identified as independent prognostic predictors.

Conclusion(s): AFP and/or DCP response at the end of a half course of HAIC may predict response and survival in advanced HCC patients undergoing HAIC.

\section{PS08-011 \\ Treatment of Hepatocellular Carcinoma in Elderly Patients \\ June Sung Lee*, Jong Wook Kim, Won Ki Bae, Nam Hoon Kim, Kyung-Ah Kim \\ Internal Medicine, Inje University Ilsanpaik Hospital, Goyang, Korea}

Introduction: The aim of this study was to compare the survival in elderly hepatocellular carcinoma (HCC) patients treated with curative modalities (radiofrequency ablation (RFA), percutaneous ethanol injection (PEIT) and surgery) to those treated with transcatheter arterial chemoembolization (TACE) and supportive care.

Methods: Medical records of patients with HCC older than 75 years who had visited a single tertiary medical center from January 2000 to December 2012 were reviewed $(n=58)$. Multivariable-adjusted hazard ratios (HR) for mortality with 95\% confidence intervals (CI) were estimated using Cox proportional hazard models.

Results: Twenty-nine patients were treated by TACE, 19 patients by supportive care, and 10 patients by curative treatment (four by PEIT, three by surgery and three by RFA). Variables associated with increased survival were better Child-Pugh class and lower TNM stage. Treatment with curative intent showed significant survival benefit compared to TACE (HR for mortality, 0.10; 95\% CI, 0.01-0.95). In a subgroup analysis among patients with resectable HCC, supportive care showed significantly worse survival over TACE (HR for mortality, 6.47; 95\% CI, 2.14-19.56) and curative intent (HR for mortality, 16.23; 95\% CI, 1.92-136.83).

Conclusion(s): Curative treatment seems to have a better survival benefit in comparison with other treatment modalities in elderly HCC patients.

\section{PS08-012 \\ The Role of Radiofrequency Ablation in Patients with Hepatocellular Carcinoma Undergoing Prior Transarterial Chemoembolization}

Ju-Yeon Cho Won Sohn, Jemma Ahn, Dong Hyun Sinn, Geum-Youn Gwak, Yong-Han Paik, Joon Hyeok Lee, Kwang Cheol Koh, Seung Woon Paik, Byung Chul Yoo, Moon Seok Choi*

Medicine, Samsung Medical Center, Seoul, Korea

Introduction: The role of radiofrequency ablation (RFA) remains uncertain in patients with recurrent hepatocellular carcinoma (HCC) after transarterial chemoembolization (TACE). This study was aimed to determine the outcome and prognostic factors after RFA in recurrent HCC undergoing prior TACE.

Methods: A total of 101 patients underwent RFA from April 2007 to August 2010 for recurrent $(n=38)$ or residual $(\mathrm{n}=68)$ HCC after TACE (median 3 sessions, range 1-14). We analyzed their long-term outcome and predictive factors affecting survival and early recurrence.

Results: Median duration between RFA and last TACE was $3.2(0.9-22.4)$ months. Overall survival rates after RFA were $93.1 \%, 65.4 \%$, and $61.0 \%$ at 1,3 , and 5 years, respectively. Predictive factors for favorable overall survival were Child-Pugh class A (hazard ratio (HR) 3.45, p=0.001), serum AFP $<20 \mathrm{ng} / \mathrm{mL}$ (HR 2.90, p = 0.02), and recurrent tumor after last TACE (HR 3.14, $\mathrm{p}=0.007$ ). Cumulative recurrence-free survival rates after RFA at 6 months were $50.1 \%$. Predictive factors for early recurrence (within 6 months) were serum AFP $\geq 20 \mathrm{ng} / \mathrm{mL}$ (HR 3.02, p < 0.001), tumor size $\geq 30 \mathrm{~mm}$ at RFA (HR 2.90, p = 0.005), and non-responder to the last TACE (HR 2.13, $\mathrm{p}=0.013$ ).

Conclusion(s): Patients with recurrent or residual HCC undergoing previous TACE show favorable overall survival, although their HCC seems to recur early and frequently. While good liver function, low serum AFP level, and recurrent tumor were independent predictive factors for favorable overall survival, poor response to TACE, high serum AFP level, and large tumor are associated with early recurrence. 


\section{PS08-015 \\ Hepatocellular Carcinoma with Bile Duct Tumor Thrombus: Consideration of Our 2 Experiences}

llyoung Park, Hojoong Choi*

Surgery, Bucheon St. Mary's Hospital, Bucheon, Korea

Introduction: Hepatocellular carcinoma (HCC) spreads along the portal vein, and then invasion of the portal vein by HCC is common, but tumor thrombi are detected within bile duct, rarely. Bile duct tumor thrombus can cause obstructive jaundice, Once a tumor thrombus completely obstructs the main bile duct, it causes obstructive jaundice. This type of HCC, called icteric-type HCC (IHCC), may be misdiagnosed as cholangiocarcinoma.

Methods: Presentation of cases: Jan. 2014, two patients were performed right hepatectomy due to HCC with bile duct tumor thrombus. The first case was 59-year-old man. He was chronic hepatitis B patient. He came to the hospital because of jaundice, and then computed tomography (CT) showed a tumor occupying right main intrahepatic bile duct. We could not distinguish HCC and cholangiocarcinoma before surgery. We performed a right hepatectomy and thrombectomy. The second case was 44-year-old man. He was also chronic hepatitis B patient. Four months prior to surgery, he was treated with TACE, but follow-up CT scan showed viable portion. Initially, he had no bile duct tumor thrombus. We also performed Rt. hepatectomy and thrombectomy.

Results: The first case revealed HCC which was found only in the bile duct. Unfortunately, we could not achieve R0 resection in the second case, because histological examination revealed remnant HCC in the bile duct of caudate process.

Conclusion(s): HCC with bile duct tumor thrombus have been reported to have a poor prognosis. The ideal treatment is complete resection of tumor including thrombus. It must be determined carefully when planning surgery for R0 resection.

\section{PS08-016 \\ Subclassification System and Recommended Treatment Options for Patients with Intermediate Stage of Hepatocellular Carcinoma}

Jae Min Lee ${ }^{1}$, Yeon Seok Seo ${ }^{1 *}$, Sun Young Yim ${ }^{1}$ Chang Ho Jung ${ }^{1}$, Tae Hyung Kim¹, Eun Sun Kim¹, Bora Keum ${ }^{1}$, Hyunggin An ${ }^{2}$, Yoon Tae Jeen ${ }^{1}$, Soon Ho Um ${ }^{1}$, Ho Sang Ryu ${ }^{1}$, Hong Sik Lee ${ }^{1}$

${ }^{1}$ Department of Internal Medicine, Korea University College of Medicine, Seoul, Korea; ${ }^{2}$ Department of Biostatics, Korea University College of Medicine, Seoul, Korea

Introduction: The intermediate stage of hepatocellular carcinoma (HCC) includes very heterogeneous population with diverse prognosis. Therefore, subclassification could be beneficial for patients in this stage by planning more appropriate treatment. This study was performed to evaluate the prognostic factors and set a subclassification system for patients with intermediate HCC.

Methods: Patients who were diagnosed as HCC between 2005 and 2013 were enrolled in this study. Patients with previous history of HCC treatment or diagnosed with other malignancies were excluded. Nine-hundred eighty patients were satisfied in these criteria. According to the BCLC staging system, 396 (40.4\%), 205 (20.9\%), 260 (26.5\%), and 119 (12.1\%) patients were classified as BCLC stage A, B, C, and D. Among 205 patients with BCLC stage B, 4 patients who had liver transplantation as the first-line treatment and 7 patients who were lost to follow-up within 1 month without any treatment for HCC were excluded. Therefore, 192 patients were analyzed in this study. Treatment was classified into 3 groups as follows: surgical resection or radiofrequency ablation, direct treatment; transarterial chemoembolization or radioembolization, transarterial treatment; best supportive care (BSC).

Results: Age was $61.1 \pm 11.7$ years and 157 patients $(81.8 \%)$ were male. Child-Pugh score was $5.9 \pm 1.2$. Number of HCC nodules were $3.5 \pm 2.5$ and it was $>3$ in 76 patients (39.6\%). Diameter of maximum nodules were $6.0 \pm 3.3 \mathrm{~cm}$ and it was $>10 \mathrm{~cm}$ in 27 patients $(14.1 \%)$. Serum AFP level was $2.1 \pm 1.4 \log \mathrm{ng} / \mathrm{mL}$. Patients were treated with direct treatment, transarterial treatment, and BSC in 104 (54.2\%), 74 (38.5\%), and 14 (7.3\%). Patients were followed for $20.1 \pm 17.5$ months. During follow-up, 52 patients were expired and 1- and 2-year survival rates were $86.7 \%$ and $72.8 \%$, respectively. On univariate analysis, number of nodules, diameter of maximum nodule, up-to-seven score, serum AFP level, Child-Pugh score, and performance status were significantly associated with mortality. On multivariate analysis, number of nodules ( $<3 \mathrm{vs}>3$; OR, 2 ; $\mathrm{P}=0.029$ ), diameter of maximum nodules $(<10 \mathrm{~cm}$ vs $>10 \mathrm{~cm}$; OR, 5; $\mathrm{P}<0.001)$, serum AFP level $(<800 \mathrm{ng} / \mathrm{mL}$ vs $>800 \mathrm{ng} / \mathrm{mL}$; OR, 3; P < 
0.001), and Child-Pugh score ( $<7$ vs 7; OR, 4; P $<0.001$ ) were independent risk factors for mortality. Patients were scored according to the $\mathrm{OR}$ of the risk factors which they had and classified according to the score as follows: stage B1, score 0; stage B2, score 1 5; stage B3, score 6 9; stage B4, score $>10$. Sixtyseven (34.9\%), 93 (48.4\%), 26 (13.5\%), and 6 (3.1\%) patients were classified as stage B1, B2, B3, and B4, respectively. Duration of survival was significantly different according to the stage: B1, $58.8 \pm 3.7$ months; B2, $46.0 \pm 4.7$ months; B3, $16.5 \pm 2.8$ months; $\mathrm{B} 4,5.3 \pm 1.8$ months $(\mathrm{P}<0.001)$. In stage $\mathrm{B} 1$, prognosis was significantly better in patients treated with direct treatment compared to transarterial treatment, while it was comparable in patients with B2 or B3.

Conclusion(s): Subclassification of patients with intermediate HCC according to the number of tumor, diameter of maximum nodule, serum AFP level, and Child-Pugh score was very useful for predicting prognosis in these patients. In patients with stage B1, surgical resection or RFA should be considered as 1st-line treatment, while patients with stage B2 or B3 could be treated with surgical resection, RFA, or transarterial treatment according to the physician's decision.

\section{PS08-017 \\ Lenalidomide as Second-Line Treatment for Advanced Hepatocellular Carcinoma (HCC): Preliminary Results of a Phase 2 Trial \\ Yu-Yun Shao ${ }^{1}$, Bang-Bin Chen ${ }^{2}$, Zhong-Zhe Lin', Chih-Hung Hsu', Miao-Jen Wang ${ }^{1}$, Ann-Lii Cheng ${ }^{1}$, Chiun $\mathrm{Hsu}^{1 *}$ \\ ${ }^{1}$ Department of Oncology, National Taiwan University Hospital, Taipei City, Taiwan; ${ }^{2}$ Department of Medical Imaging, National Taiwan University Hospital, Taipei City, Taiwan}

Introduction: Lenalidomide has both immune-modulatory and anti-angiogenic activity. It was shown to have antitumor efficacy for advanced HCC as second-line treatment in a Western population. This study sought to confirm its efficacy and safety in an Asian Population.

Methods: Eligible criteria included histological or clinical diagnosis of HCC, documented progression with or intolerance to sorafenib-based treatment or other anti-angiogenic therapy as first-line therapy for advanced HCC, ECOG score 0 or 1 , Child class A, and measurable tumors by RECIST 1.1. All enrolled patients received lenalidomide, starting at $25 \mathrm{mg}$ orally daily on days $1-21$, every 4 weeks. Dose titration was done according to the severity of adverse events. Tumor response was assessed after 4 weeks and 8 weeks of treatment and every 8 weeks thereafter until objective disease progression. The primary endpoint was the 6-month progression-free survival (PFS) (according to RECIST 1.1) after lenalidomide treatment.
Results: From September 2012, 22 patients were enrolled (M: F 16:6; median age 57.5 years (range 31.0-79.8 years); HBV/HCV 21/4 (3 had dual infection); median alpha fetoprotein (AFP) $858.4 \mathrm{ng} / \mathrm{mL}$ (range 1.9 - >80000); CLIP score $0-1 / 2 / 3 / 46 / 6 / 7 / 3$; extra-hepatic metastases 20 patients). All but one patient had documented progression after prior sorafenib treatment. Three partial responses and 9 stable diseases were documented in 21 evaluable patients, accounting for a response rate of $14 \%$ (95\% C.I. 0-31\%). Only one patient remained progression-free at 6 months $(4.8 \%$, 95\% C.I. 0-14.7\%). As of 10 April 2014, the median PFS and overall survival was 12.0 weeks (95\% C.I. 3.0-21.0) and 32.3 weeks (95\% C.I. 28.1-36.5), respectively. One patient died of pneumonia. Grade 3/4 adverse events occurring in $>10 \%$ of patients included neutropenia ( 5 patients) and transaminase elevation (3 patients).

Conclusion(s): Lenalidomide is well tolerated in HCC patients. Combination therapy warrants further investigation.

\section{PS08-018 \\ Long-Term Follow-Up of Patients with Advanced Hepatocellular Carcinoma Who Achieved Complete Response after Sorafenib Therapy: Case Series in Korean-Multicenter Cohort}

Jung Gil Park' ${ }^{1}$, Soo Young Park ${ }^{2 *}$, Young Oh Kweon', Won Young Tak ${ }^{2}$, Se Young Jang ${ }^{2}$, Su Hyun Lee ${ }^{2}$, Yu Rim Lee $e^{2}$, Jeong $\mathrm{Heo}^{3}$, Hyun Young Woo ${ }^{3}$

${ }^{1}$ Internal medicine, CHA University Gumi CHA Medical Center, Gumi, Korea; ${ }^{2}$ Internal medicine, Kyungpook National University Hospital, Daegu, Korea; ${ }^{3}$ Internal medicine, Pusan National University Hospital, Pusan, Korea

Introduction: Sorafenib is currently the sole systemic chemotherapeutic agent that improves overall survival of advanced hepatocellular carcinoma (HCC). Despite the efficacy of sorafenib, response rate was varied in patient with advanced HCC. We analyzed retrospective series of complete response after sorafenib therapy in patients with advanced HCC in Korea.

Methods: Sorafenib was given initially at a dose of $400 \mathrm{mg}$ orally, twice daily. Tumor response and recurrence rates were assessed by radiologic study and tumor marker.

Results: Five patients with advanced HCC were treated with sorafenib. Median period of tumor-disappearance and observed disease-free was 5.5 months and 22 months, respectively. Four patients continued sorafenib after complete response. From the entire cohort, recurrence of HCC was identified in two cases. Of these, one patient early discontinued sorafenib. The patients recurred at the time of 2.4 and 42 months after complete response. Three patients needed dose reduction for toxicity and adverse events. 
Conclusion(s): Though completeresponse after sorafenib therapy was achieved in patients with advanced HCC, recurrence rate was relatively high. Subsequent treatments after sorafenib therapy are required to offer the chance for a cure. Further studies should be needed to identify the molecular features of these tumors.

\section{PS08-019 \\ Real-Life Data of Sorafenib in Unresectable Hepatocellular Carcinoma: Retrospective Analysis of 511 Consecutive Patients Treated with Sorafenib in a Single Tertiary Referral Center}

Yeonjung $\mathrm{Ha}^{1}$, Kang Mo Kim ${ }^{1 *}$, Sook Ryun Park ${ }^{2}$, Min-Hee Ryu' ${ }^{2}$, Baek-Yeol Ryoo ${ }^{2}$, Yoon-Koo Kang ${ }^{2}$, Danbi Lee ${ }^{1}$, Ju Hyun Shim1, Young-Suk Lim¹, Han Chu Lee ${ }^{1}$, Young-Hwa Chung ${ }^{1}$, Yung Sang Lee ${ }^{1}$

${ }^{1}$ Department of Gastroenterology, Asan Liver Center, Asan Medical Center, University of Ulsan College of Medicine, Seoul, Korea; ${ }^{2}$ Department of Oncology, Asan Medical Center, University of Ulsan College of Medicine, Seoul, Korea

Introduction: Real-life data of sorafenib could be different from that of clinical trials. This study aimed to characterize patients treated with sorafenib and to analyze the practice pattern

Methods: Patients to whom sorafenib was prescribed from 2007 to 2009 in Asan Medical Center were identified. Baseline characteristics, sorafenib dosing, treatment duration, concomitant treatment, and the reason(s) for sorafenib termination were evaluated. Overall survival (OS) and progressionfree survival (PFS) were also calculated.

Results: 511 patients (mean age 53 years, $86.1 \%$ male) were analyzed. At the time of sorafenib initiation, tumor stages were Tumor-Node-Metastasis (TNM) III in 8.0\% and IV in $91.4 \%$ of patients, whereas $1.1 \%$ and $98.3 \%$ fell into Barcelona Clinic Liver Cancer (BCLC) stage B and C, respectively. Liver function was Child-Pugh A in $77.9 \%$ of patients, and the remaining $21.4 \%$ and $0.7 \%$ were Child-Pugh B and C. Most patients (88.0\%) showed distant metastasis at sorafenib initiation, and $73.8 \%$ among those without metastasis started sorafenib due to portal vein invasion. Mean time since first indication to the actual initiation of treatment was $13.2 \pm 96.3$ months. Average dose of sorafenib through the treatment period was $660.2 \pm 159.3 \mathrm{mg} /$ day and mean duration of treatment was $3.9 \pm 6.2$ months. $72.5 \%$ of patients were treated with sorafenib alone, while others received concomitant therapy with transarterial chemoembolization (15.7\%), radiation therapy $(6.7 \%)$, or both $(5.5 \%) .66$ patients $(12.9 \%)$ withdrew from treatment because of adverse events. Other reasons of stopping sorafenib were death or disease progression (65.5\%), managing physicians' decision (7.2\%), and economic issue (4.9\%). The median OS and PFS were $8.3(6.2-10.4)$ months and 2.5 (1.6-3.5) months, respectively.

Conclusion(s): Patients treated with sorafenib for unresectable HCC in Asan Medical Center mostly had TNM stage IV, BCLC stage $C$ disease and Child-Pugh status A. Tolerance to sorafenib was better than previous studies.

\section{PS08-020 \\ Single Nucleotide Polymorphisms Are Associated with the Outcome of Chemotherapy in Advanced Hepatocellular Carcinoma}

Wey-Ran Lin, Chau-Ting Yeh*

Liver Research Unit, Linkou Chang Gung Memorial

Hospital, Taoyuan, Taiwan

Introduction: Besides targeted agents, chemotherapy is an option for advanced hepatocellular carcinoma (HCC) but profound side effects hinder its clinical use. A pilot genomewide association study was performed in HCC patients receiving 5-fluorouracil, mitoxantrone and cisplatin (FMP) combination chemotherapy. An SNP located at the intron of GALNT14 has been validated to be associated with the therapeutic responses and outcomes of chemotherapy (Int J Cancer 2014). Several candidate SNP markers including rs6025211 (near BMP7), rs715171 (near FAM9B; X-linked), rs1955024 (intergenic) and rs13338697 (near WWOX) were also identified in the pilot GWAS. This study aims to examine the prognostic value of these candidate markers in an independent cohort of 116 patients.

Methods: 116 patients with HCC receiving split-dose FMP were enrolled. The clinical parameters and rs6025211, rs715171, rs1955024 and rs13338697 genotypes were analyzed. The independent predictors for time-to-progression (TTP) and overall survival (OS) were assessed using Cox proportional hazards models. After categorization, KaplanMeier method with log-rank test was used to compare the survivals.

Results: In univariate analysis of SNP markers, GALNT14 "TT" genotype was identified as a favorable prognostic marker for both TTP and OS (P = 0.012 and 0.002). rs13338697 "CT" genotype was associated with an unfavorable TTP $(\mathrm{P}=0.040)$, and rs6025211 "CT " genotype was associated with an unfavorable OS $(\mathrm{P}=0.014)$. In this cohort, 50 patients with rs13338697 "CT" genotype had a shorter median TTP (1.96 versus 2.75 months, $P=0.038) .48$ patients with rs6025211 "CT " had a shorter median OS (3.89 versus 5.39 months, $\mathrm{P}=$ 0.013). 29 patients with GALNT14 "TT" genotype had a longer median TTP (3.11 versus 2.11 months, $\mathrm{P}=0.011$ ) and a longer median OS (5.75 versus 3.89 months, $\mathrm{P}=0.002)$, when compared with the "non-TT" genotype. In multivariate analysis including both clinical parameters and SNP markers, 
only GALNT14 was identified as a favorable predictor for both TTP and OS ( $\mathrm{P}=0.003$ and 0.008$)$, suggesting that $\mathrm{rs} 13338697$ and rs6025211 genotypes were likely associated with the clinical prognostic predictors.

Conclusion(s): The rs13338697 and rs6025211 genotypes were SNP markers associated with clinical prognostic predictors. These two markers could assist GALNT14 genotype to identify a subgroup of patients with excellent prognosis.

\section{PS08-021 \\ Inhibition of WNT/B-Catenin Signaling Pathway Improves the Anti-Tumor Effect of Sorafenib against Hepatocellular Carcinoma \\ Hsiao-Hui Lin ${ }^{1}$, Wen-Chi Feng ${ }^{2}$, Li-Chun Lu', Yu-Yun Shao ${ }^{1}$, Ann-Lii Cheng ${ }^{3}$, Chih-Hung Hsu ${ }^{1 *}$ \\ ${ }^{1}$ Department of Oncology, National Taiwan University Hospital, Taipei, Taiwan; ${ }^{2}$ Graduate Institute of Biochemistry and Molecular Biology, National Taiwan University College of Medicine, Taipei, Taiwan; ${ }^{3}$ Departments of Oncology and Internal Medicine, National Taiwan University College of Medicine and National Taiwan University Hospital, Taipei, Taiwan}

Introduction: Sorafenib, a multikinase inhibitor that targets primarily Raf kinase and vascular endothelial growth factor receptor, is currently the only approved agent for patients with advanced hepatocellular carcinoma (HCC). Aberrant activation of WNT/ $\beta$-catenin signaling pathway, which plays an important role in hepatocarcinogenesis, has been shown to mediate the resistance to various types of anticancer therapy. We hypothesized that inhibition of WNT/ $\beta$ catenin signaling pathway would improve the anti-tumor effect of sorafenib in HCC.

Methods: Human HCC cells, including Huh7, HepG2, PLC5, and Hep3B HCC cells, were treated with sorafenib with or without ICG-001 which down-regulates $\beta$-catenin/TCF signaling by specifically binding to cyclic AMP response element-binding protein. Cell viability was assessed by MTT assay, induction of apoptosis was quantitated by subG1 analysis using propidium iodide staining and Apoptosis ELISA assay (Roche, Indianapolis, IN, USA), and the expression of $\beta$-catenin and related downstream molecules was determined by Western blotting. To validate the impact of WNT $/ \beta$-catenin pathway activity on sorafenib sensitivity, downregulating $\beta$-catenin by RNA interference and overexpressing $\beta$-catenin by transfection with pcDNA3-human $\beta$-catenin were performed in Huh7 cells. Finally, the in vivo efficacy of sorafenib in combination with ICG-001 was studied in immunocompromised mice transplanted with Huh7 xenograft subcutaneously.

Results: In all HCC cells, ICG-001 enhanced the antiproliferative effect of sorafenib dose-dependently, and the combination of ICG-001 and sorafenib showed a synergistic anti-proliferative effect by median effect analysis. The addition of ICG-001 significantly increased sorafenib-induced apoptosis in HCC cells. Evaluation of a panel of pro- and anti-apoptotic molecules revealed that downregulation of Mcl-1 is the key and consistent event across different HCC cells treated with the combination of ICG-001 and sorafenib. Downregulation of $\beta$-catenin by small interfering RNA or short hairpin RNA (shRNA) increased the sensitivity to sorafenib, whereas overexpression of $\beta$-catenin decreased the sensitivity to sorafenib in Huh7 cells. Furthermore, the sorafenib-sensitization effect by shRNA-mediated $\beta$-catenin downregulation in Huh7 cells was offset by $\beta$-catenin overexpression. Finally, ICG-001 at the dose of $150 \mathrm{mg} / \mathrm{kg} / \mathrm{d}$, ip, 3 times a week in combination of sorafenib at the dose of $30 \mathrm{mg} / \mathrm{kg} /$ day, po, 5 times a week showed a more significantly growth-retarding effect in Huh7 xenograft than either drug alone.

Conclusion(s): Inhibition of $\mathrm{WNT} / \beta$-catenin signaling pathway improves the antitumor effect of sorafenib against HCC in vitro and in vivo.

\section{PS08-022 \\ Prognostic Factors of Treatment Failure in Acute Variceal Hemorrhage Occurred in Patients with Hepatocellular Carcinoma}

\author{
Hyun-Woo Park ${ }^{1}$, Soo-Young Park ${ }^{1 *}$, Yoo-Rim Lee 1 , \\ Se-Young Jang ${ }^{1}$, Su-Hyung Lee ${ }^{1}$, Won-Young Tak', \\ Young-Oh Kweon ${ }^{1}$, Jung-Gil Park ${ }^{2}$, Sun-Young Ahn ${ }^{3}$ \\ ${ }^{1}$ Department of Internal Medicine, Kyungpook National \\ University Hospital, Daegu, Korea; ${ }^{2}$ Department of \\ Internal Medicine, Cha University Hospital, Kumi, Korea; \\ ${ }^{3}$ Department of Internal Medicine, Daegu Medical \\ Center, Daegu, Korea
}

Introduction: A large proportion of patients with hepatocellular carcinoma have underlying portal hypertension which is responsible for high mortality in these patients. Although variceal bleeding is an important complication of portal hypertension seriously affecting the mortality and morbidity in HCC, there are few studies evaluating the factors related to outcomes of these patients. We evaluated the clinical characteristics, treatment outcomes of acute variceal bleeding in patients with hepatocellular carcinoma and determined the prognostic factors for treatment failure in patients with HCC.

Methods: We prospectively collected the data of patients who visited Kyungpook National University Hospital for variceal bleeding from 2009 to 2011. A total of 212 patients were collected. We analyzed 80 patients with HCC. Their clinical outcomes were compared to those who did not have HCC and a multivariate analysis was performed to identify prognostic factors for treatment failure defined in Baveno criteria V.

Results: There were no significant baseline characteristics between two patients groups. The rate of treatment 


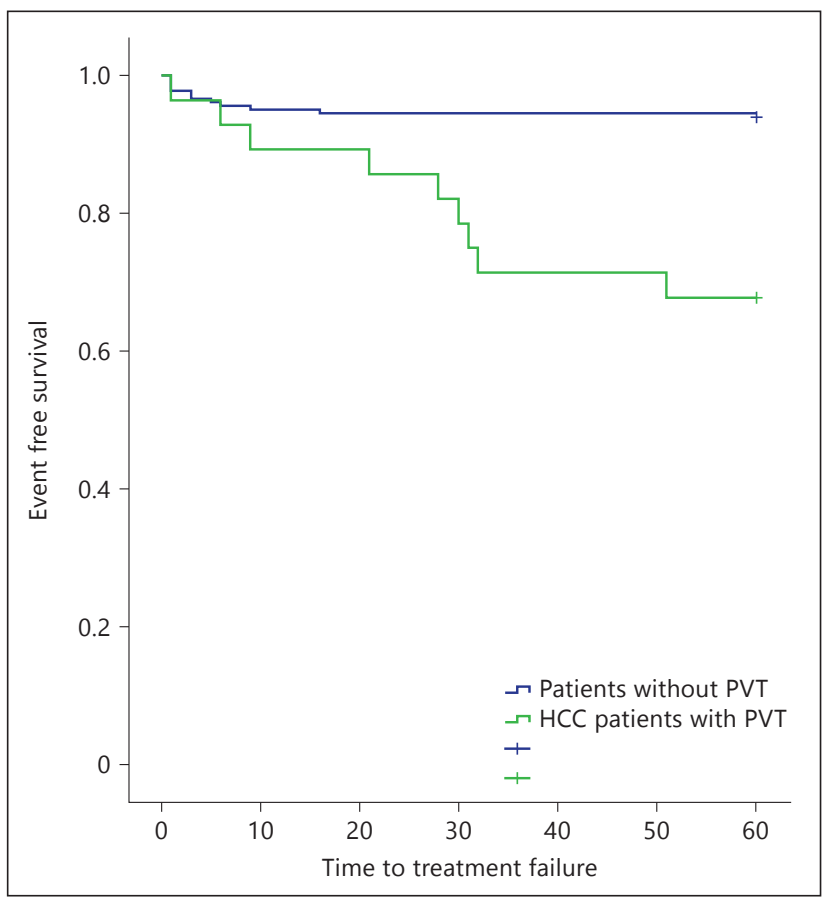

Fig. 1. (for abstract PS08-022).

failure was $15 \%$ (12 patients) in HCC patients and 6.1\% (8 patients) in non-HCC patients $(\mathrm{p}=0.049)$. The mortality rate within 60 days was $24 \%$ in HCC patients and $9 \%$ in non-HCC patients. The factors which significantly affects treatment failure were Child Pugh Classification ( $\mathrm{p}<0.001$, HR $=4.60$ and 95\% CI 2.018-10.520), MELD score ( $p=0.017$, HR 1.107 and $95 \%$ CI 1.018-1.203), shock at initial presentation ( $\mathrm{p}=$ $0.028, \mathrm{HR}=2.88$ and $95 \%$ CI 1.120-7.419), antibiotics prophylaxis ( $\mathrm{p}=0.007, \mathrm{HR}=0.275$ and 95\% CI 0.107-0.703), failure to control bleeding by endoscopy $(\mathrm{p}<0.001, \mathrm{HR}=20.143$ and 95\% CI 5.084-79.806), HCC with PVT ( $p<0.001, \mathrm{HR}=7.400$ and 95\% CI 2.724-20.139). In multivariate analysis, HCC with PVT $(\mathrm{p}=0.005, \mathrm{HR}=5.712$ and 95\% CI 1.686-19.355), failure to control bleeding by endoscopy $(\mathrm{p}=0.004, \mathrm{HR}=17.547$, and 95\% CI 2.498-123.258), antibiotics prophylaxis ( $\mathrm{p}=0.022$, HR 0.259 , and 95\% CI $0.081-0.824$ ) were factors which affected the treatment outcome in these patients.

Conclusion(s): Treatment outcomes are different between HCC patients with non-HCC patients. Factors that adversely affect the treatment outcomes of acute variceal bleeding in these patients include HCC with PVT, failure to control bleeding by endoscopy and antibiotics prophylaxis.

\section{PS08-024 \\ Treatment Patterns in $>\mathbf{3 0 0 0}$ Sorafenib- Treated Patients: Final Analysis of Gideon (Global Investigation of Therapeutic Decisions in Hepatocellular Carcinoma and of Its Treatment with Sorafenib)}

Sheng-Long $\mathrm{Ye}^{1 *}$, Riccardo Lencioni ${ }^{2}$, Jorge A. Marrero ${ }^{3}$, Alan P. Venook ${ }^{4}$, Keiko U. Nakajima ${ }^{5}$, Masatoshi Kudo 6

${ }^{1}$ Liver Cancer Institute, Zhongshan Hospital, Fudan University, Shanghai, China; ${ }^{2}$ Division of Diagnostic Imaging and Intervention, Department of Liver Transplantation, Hepatology and Infectious Diseases, Pisa University School of Medicine, Pisa, Italy; ${ }^{3}$ Department of Internal Medicine, Division of Digestive and Liver Diseases, University of Texas Southwestern Medical Center, Dallas, United States; ${ }^{4}$ Department of Medicine, University of California, San Francisco, United States; ${ }^{5}$ Global Medical Affairs Oncology, Bayer HealthCare Pharmaceuticals, Whippany, United States; ${ }^{6}$ Department of Gastroenterology and Hepatology, Kinki University School of Medicine, Osaka, Japan

Introduction: Sorafenib was approved for use in patients with unresectable hepatocellular carcinoma (uHCC) after two Phase III trials (SHARP and Asia-Pacific) demonstrated significant improvements in overall survival in uHCC patients. The recommended dose of sorafenib is $800 \mathrm{mg} /$ day. GIDEON is a prospective, observational study that evaluated the use of sorafenib in clinical practice in 39 countries establishing a large database of information on treatment practices and safety in sorafenib-treated uHCC patients.

Methods: Patients with diagnosed HCC who were candidates for systemic therapy and for whom a decision to treat with sorafenib had been made in clinical practice were eligible. Patients were enrolled from 5 regions: Asia-Pacific, Japan, Europe, USA, and Latin America. Baseline characteristics were recorded at study entry, and adverse events, dosing, and outcomes data were collected during follow-up.

Results: 3202 patients were evaluable for safety. The majority $(70 \%)$ of patients received the approved initial sorafenib dose of $800 \mathrm{mg}$. The safety profile of sorafenib was similar between patients receiving an initial dose of $800 \mathrm{mg}$ and those receiving a lower initial dose of $400 \mathrm{mg}$ (Table). The proportion of patients receiving an initial dose of $800 \mathrm{mg}$ was similarin Child-PughAandChild-Pugh Bpatients (72\%and 70\%, respectively), although a slightly lower proportion of Barcelona Clinic Liver Cancer (BCLC) A patients (62\%) received an initial dose of 800 mg compared withBCLCB(70\%) andBCLCCpatients (74\%). In Asia-Pacific, 77\% of patients received an initial dose of $800 \mathrm{mg}$, comparedwith 54\% in theUSAand 46\% inJapan. Median daily dose was higher in Asia-Pacific ( $800 \mathrm{mg}$ ) than in the USA (527 mg) and Japan (419 mg). Overall, median duration of treatment was 15 weeks, and this tended to belonger in patients who received an initial dose of $800 \mathrm{mg}$ (16 weeks) than in those receiving an initial dose of $400 \mathrm{mg}$ (12 weeks). Patients in Asia- 
Pacifichadamedianduration oftreatmentof13weekscompared with 16 weeks in Japan; however, variation was observed at the countrylevel, withpatientsinChinahavingdurationoftreatment of 22 weeks compared with only 9 weeks in Korea. Overall, $29 \%$ of patients continued sorafenib treatment for $>28$ weeks.

Conclusion(s): In the overall population, the safety profile of sorafenib was generally consistent irrespective of initial sorafenib dose. Differences in initial dose did not appear to be based on disease characteristics. Regional differences in sorafenib dosing were observed; patients in Japan tended to receive a lower initial and median sorafenib dose compared with those in Asia-Pacific.

\section{PS08-025}

Phase 2 Study of Combined Sorafenib and Radiation Therapy in Patients with Advanced Hepatocellular Carcinoma

\section{Shang-Wen Chen 1,2,3, Li-Ching Lin ${ }^{2,4}, Y u$-Cheng Kuo ${ }^{1,5}$, Ji-An Liang 1,3, Chia-Chun Kuo ${ }^{6}$, Jeng-Fong Chiou, ${ }^{2,6 *}$ \\ ${ }^{1}$ Department of Radiation Oncology, China Medical University Hospital, Taichung, Taiwan; ${ }^{2}$ School of Medicine, Taipei Medical University, Taipei, Taiwan; ${ }^{3}$ School of Medicine, China Medical University, Taichung, Taiwan; ${ }^{4}$ Department of Radiation Oncology, Chi-Mei Hospital, Tainan, Taiwan; ${ }^{5}$ Department of Biomedical Imaging and Radiological Science, China Medical University, Taichung, Taiwan; ${ }^{6}$ Department of Radiation Oncology, Taipei Medical University Hospital, Taipei, Taiwan}

Introduction: This phase 2 study evaluated the efficacy of radiation therapy (RT) with concurrent and sequential sorafenib therapy in patients with unresectable hepatocellular carcinoma (HCC).
Methods: Forty patients with unresectable HCC unfit for transarterial chemoembolization were treated with RT with concurrent and sequential sorafenib. Sorafenib was administered from the commencement of RT at a dose of $400 \mathrm{mg}$ twice daily and continued to clinical or radiologic progression, unacceptable adverse events, or death. All patients had underlying Child-Pugh A cirrhosis. The maximal tumor diameter ranged from $3.0 \mathrm{~cm}$ to $15.5 \mathrm{~cm}$. Coexisting portal vein thrombosis was found in 24 patients and was irradiated simultaneously. The cumulative RT dose ranged from 40 Gy to 60 Gy (median, 50 Gy). Image studies were done 1 month after RT and then every 3 months thereafter.

Results: Thirty-three (83\%) completed the allocated RT. During RT, the incidence of hand-foot skin reactions $\geq$ grade 2 and diarrhea were $37.5 \%$ and $25 \%$, respectively, and $35 \%$ of patients had hepatic toxicities grade $\geq 2$. Twenty-two (55.0\%) patients achieved complete or partial remission at the initial assessment, and 18 (45\%) had stable or progressive disease. The 2-year overall survival and infield progression free survival (IFPS) were 32\% and 39\%, respectively. A Cancer of the Liver Italian Program (CLIP) score $\geq 2$ was associated with an inferior outcome in overall survival. Six patients (15\%) developed treatment-related hepatic toxicity grade $\geq 3$ during the sequential phase, and 3 of them were fatal.

Conclusion(s): When RT and sorafenib therapy were combined in patients with unresectable HCC, the initial complete or partial response rate was $55 \%$ with a 2 -year IFPS of $39 \%$. A CLIP score $\geq 2$ was associated with an inferior outcome in overall survival. Hepatic toxicities are a major determinant of the safety; the combination should be used with caution and needs further investigation.

\begin{tabular}{|lccc|}
\hline & \multicolumn{2}{c|}{ Initial sorafenib dose } & \\
\cline { 2 - 3 }$\%$ & $\begin{array}{c}400 \mathrm{mg} / \text { day } \\
(\mathrm{n}=821)\end{array}$ & $\begin{array}{c}800 \mathrm{mg} / \mathrm{day} \\
(\mathrm{n}=2,251)\end{array}$ & $\begin{array}{c}\text { Totala } \\
(\mathrm{n}=3,202)\end{array}$ \\
\hline AEs (all grades) & 92.4 & 82.7 & 85.3 \\
Drug-related AEs (all grades) & 74.2 & 63.6 & 66.0 \\
SAEs (all grades) & 47.7 & 41.4 & 43.3 \\
Drug-related SAEs (all grades) & 10.6 & 8.8 & 9.3 \\
All grade 3 or 4 & 35.4 & 30.2 & 31.8 \\
Drug-related grades 3 or 4 & 25.1 & 23.1 & 23.6 \\
$\begin{array}{l}\text { Any AE resulting in permanent } \\
\text { discontinuation of sorafenib }\end{array}$ & 36.9 & 29.2 & 31.4 \\
\hline
\end{tabular}

Fig. 1. ${ }^{\text {a }}$ Total includes patients receiving initial sorafenib doses other than $400 \mathrm{mg} /$ day or $800 \mathrm{mg} /$ day; ${ }^{b}$ Any AE occurring at any dose that results in any of the following outcomes: death; life-threatening; hospitalization or prolongation of existing hospitalization; persistent or significant disability/ incapacity; congenital anomaly/birth defect; medically important event. $\mathrm{AE}$ = Adverse event; $\mathrm{SAE}=$ serious adverse event (for abstract PS08-024). 


\section{Category 9 \\ Controversies for the Locally Advanced HCC}

\begin{abstract}
PS09-002
Clinical Effectiveness and Outcomes of Proton Beam Therapy for Advanced Hepatocellular Carcinoma Patients with Portal Vein Tumor Thrombosis

Sung Uk Lee, Joong-Won Park, Tae Hyun Kim*, Yeon-Joo Kim, Bo Hyun Kim, Sang Myung Woo, Young-Hwan Koh, Woo Jin Lee, Sang-Jae Park, Dae Yong Kim, Chang-Min Kim

Center for Liver Cancer, National Cancer Center, Goyang, Korea
\end{abstract}

Introduction: To evaluate the clinical effectiveness and safety of proton beam therapy (PBT) in advanced hepatocellular carcinoma (HCC) patients with portal vein tumor thrombosis (PVTT).

Methods: Twenty-seven HCC patients with PVTT underwent PBT, including 22 with modified International Union Against Cancer (mUICC) stage IVA and 5 with stage IVB primary tumors and 16 with main PVTT. A median dose of 55 GyE (range, 50-66 GyE) in 20-22 fractions was delivered to a target volume encompassing both the PVTT and primary tumor.

Results: Overall, treatment was well tolerated, with no grade $\geq 3$ toxicity. Median overall survival (OS) times in all and stage IVA patients were 13.2 months and 16 months, respectively. Assessments of PVTT response showed complete response in 0 of $27(0 \%)$ patients, partial response in 15 (55.6\%), stable disease in 10 (37\%), and progressive disease in $2(7.4 \%)$ patients, with an objective response rate of $55.6 \%$. PVTT responders showed significantly higher actuarial 1-year local progression-free survival (LPFS) (85.6\% vs. 51.3\%), relapse-free survival (RFS) (20\% vs. $0 \%)$ and OS (80\% vs. $25 \%$ ) rates than non-responders ( $\mathrm{p}<0.05$ each). Multivariate analysis showed that PVTT response and mUICC stage were independent prognostic factors for OS.

Conclusion(s): Our data suggest that PBT could improve LPFS, RFS and OS in advanced HCC patients with PVTT and it is feasible and safe for these patients.

\author{
PS09-003 \\ An Appraisal of Liver Resection and \\ Thrombectomy for Hepatocellular Carcinoma \\ with Tumor Thrombi Extending over Portal \\ Vein Bifurcation \\ Cheng-Chung $W^{1}{ }^{1}$, Cheng-Chung $W u^{1}$, \\ Shao-Bin Cheng', Cheng-Chan Yu', John Wang ${ }^{2 *}$ \\ ${ }^{1}$ Surgery, Taichung Veterans General Hospital, \\ Taichung, Taiwan; ${ }^{2}$ Pathology, Taichung Veterans \\ General Hospital, Taichung, Taiwan
}

Introduction: The prognosis of hepatocellular carcinoma (HCC) with gross tumor thrombi (TT) extending to or over portal vein bifurcation is usually poor. Transarterial chemoembolization (TACE) or liver transplantation is always unrecommended due to risk of liver failure or early cancer recurrence. The aim of the study is to evaluate the benefit of liver resection for such tumor.

Methods: A retrospective review of 85 liver resections for newly diagnosed HCC with tumor thrombi extending to or over portal vein bifurcation between 1991 and 2012 was conducted. In the similar time, 2094 major hepatectomy, liver resection for HCC were performed. The patients' age was 64.8+12.9 (range 21-86 yrs) (right or extended right hepatectomy, left or extended left hepatectomy) were performed on 48 patients. Partial hepatectomies with TT removal were performed on the other 37 patients due to less favorable liver function.

Results: Postoperative complication occurred in 22 patients $(25.8 \%)$ without 90-day postoperative mortality. Five-year disease-free and overall survival rates are $21.6 \%$ and $27 \%$, respectively. No difference of survival rates would be seen in major hepatectomy and partial hepatectomy. After univariate and multivariate analysis only tumor size $>10 \mathrm{~cm}$ had a poorer prognosis than tumor $<10 \mathrm{~cm}(\mathrm{p}<0.0001)$. 5-year survival rate for tumor $>10 \mathrm{~cm}$ and $<10 \mathrm{~cm}$ were $11.8 \%$ and $43.6 \%$ respectively. The longest disease-free survival time in this series is 19 years.

Conclusion(s): Liver resection with portal vein thrombi removal may provide possible cure for HCC with tumor thrombi extending over portal vein bifurcation. However, adjuvant therapies should be considered when tumor size is $>10 \mathrm{~cm}$. 
The 5th Asia-Pacific Primary Liver Cancer Expert Meeting (APPLE 2014)

\section{PS09-004 \\ 4D Ultrasonogaraphy for Therapeutic Radiofrequency Ablation for Hepatocellular Carcinoma \\ Naoki Hotta*}

Department of Hepatology, Masuko Memorial Hospital, Nagoya, Japan

Introduction: Studies to evaluate the tumor vascularity in HCC have been done extensively with various imaging modalities because the finding of the vasularity is helpful to evaluate the biological features of the tumor. In the present study, we investigated whether $4 \mathrm{D}$ real-time flow imaging is useful to display the accurate position of percutaneous radiofrequency ablation (RFA) needle in the tumor and evaluated the efficacy of RFA therapy in patients with HCC

Methods: 58 patients with 58 HCC lesions (44 men and14 women, aged 40 to 83 years with a mean age of 61.9 years), admitted to our Masuko Memorial Hospital between November 2007 and February 2011, were enrolled to the present study. Their diagnosis was confirmed by dynamic CT and celiac angiography. Based on Child-Pugh score, 50 patients was diagnosed as grade $\mathrm{A}$, and 8 patients as grade B. All patients enrolled showed hypervascular enhancement of HCC on contrast-enhanced US and/or dynamic CT. The diameters of tumors were $1.1-2.0 \mathrm{~cm}$ in 30 nodules, $2.1-3.0 \mathrm{~cm}$ in $19,3.1-5.0 \mathrm{~cm}$ in 9, respectively. All patients gave written informed consent and this protocol had been approved by the Human Studies Committee at Masuko Memorial Hospital. US imaging We used VOLUSON730 (GE Medical systems, Milwaukee), APLIO XG (Toshiba Medical Systems) and IU22 (Phillips) for RFA therapy with a convex probe as US system. APLIO and VOLUSON machine probe is mechanical probe and IU22 probe is matrix array probe. $4 \mathrm{D}$ Real-time refers here to the display of 3-dimensional moving images composed of 3 orthogonally intersecting scans in the transverse, longitunal and horizontal planes. RF ablation was carried out under a real-time US guidance. We used 30 patients a radiofrequency generator with $200 \mathrm{~W}$ power connected to a 17-gauge perfusion needle (Radionics Inc., Burlington, MA) the circuit was closed through a dispersive electrode and 28 patients used LeVeen Needle (Boston Scientific).

Results: It was possible to obtain accurate position of cool-tip needle and to perform RFA procedure in all 58 HCC patients with 58 nodules using 4D real-time US machines. We confirmed by various angles that the needle was inserted into the center of tumor nodule. The simultaneous study before RFA therapy showed the inflow of arterial blood and tumor stain And importantly it appeared that 4D real-time US provided much perceptible information on the spatial relationship between RFA needle and the target lesion, and resulted in accurate We experienced the treatment of 58 patient with HCC by RFA using 4D real-time ultrasound

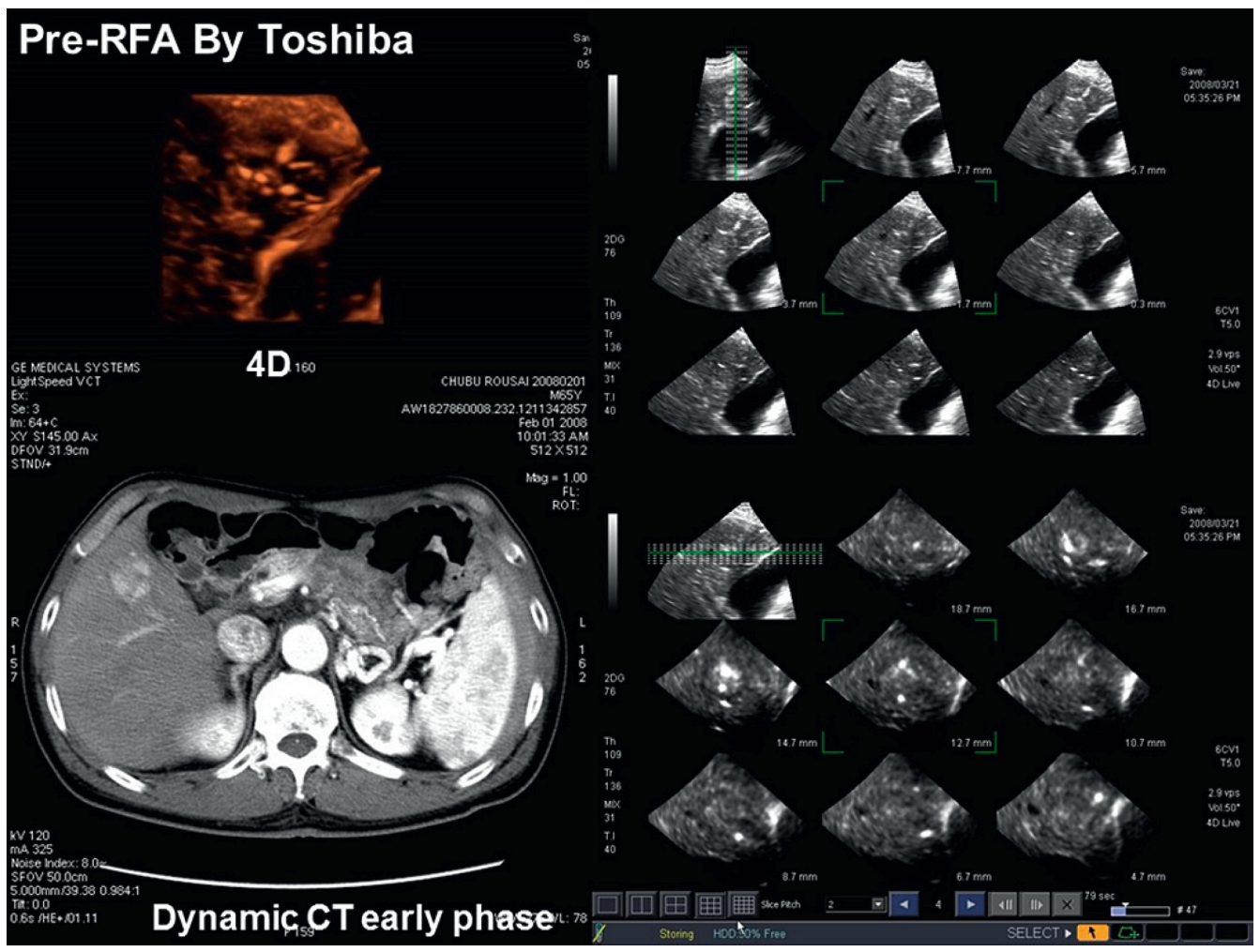

Fig. 1. APLIO (for abstract PS09-004). 


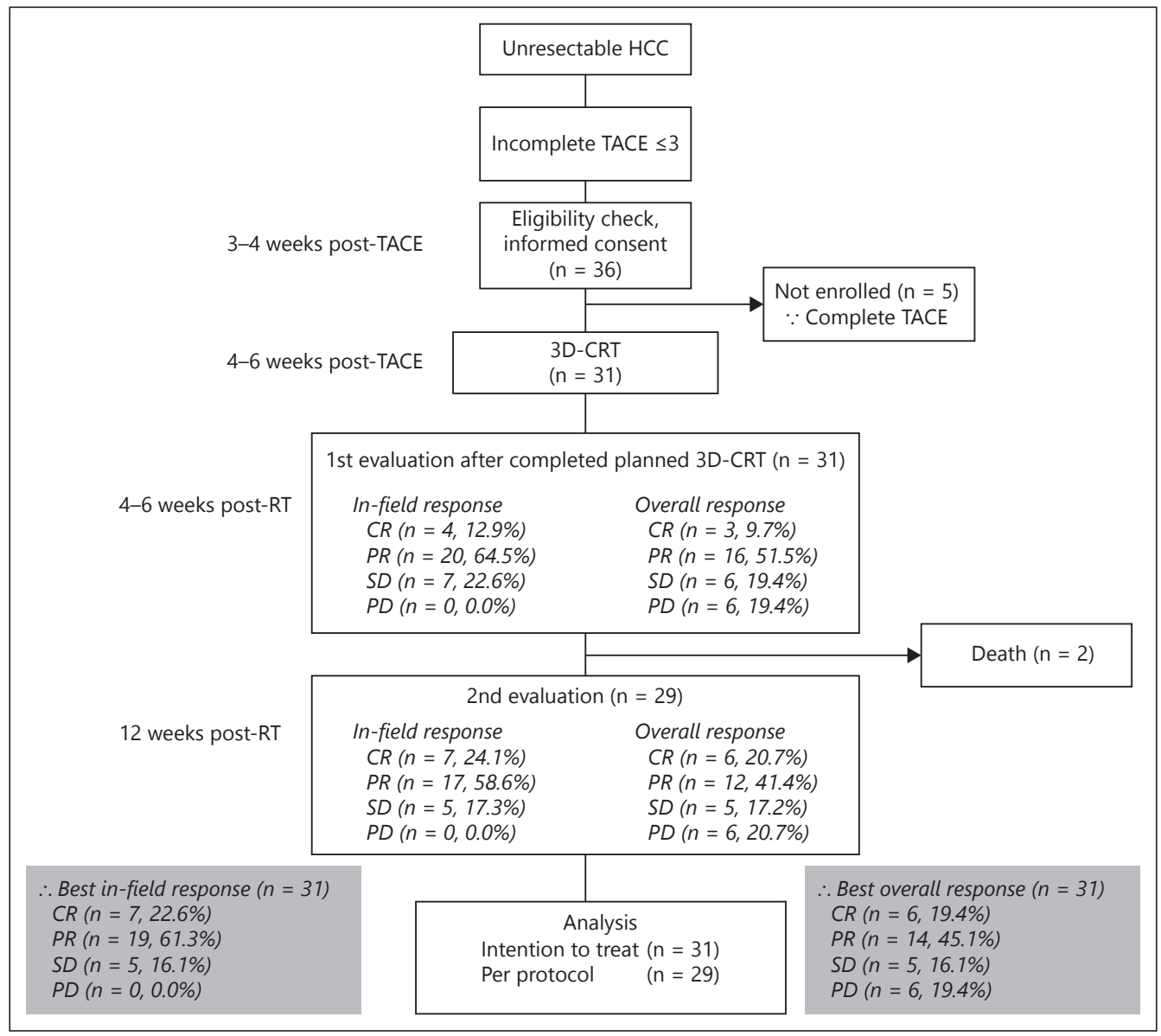

Fig. 1. Flow diagram (for abstract PS09-005).

system. Application of this menthod allowed a more accuraye cauterization of the tumor.

Conclusion(s): We experienced the treatment of 58 patients with HCC by RFA using 4D real-time ultrasound system. Application of this menthod allowed a more accuraye cauterization of the tumor.

\section{PS09-005}

\section{A Prospective Phase li Multicenter Study for the Efficacy of Radiotherapy Following Incomplete Transarterial Chemoembolization in Unresectable Hepatocellular Carcinoma}

Chihwan Choi ${ }^{1}$, Woong Sub Koom ${ }^{1}$, Tae Hyun Kim², Sang Min Yoon ${ }^{3}$, Jin Hee Kim ${ }^{4}$, Hyung-Sik Lee ${ }^{5}$, Taek-Keun Nam ${ }^{6}$, Jinsil Seong ${ }^{1 *}$

${ }^{1}$ Department of Radiation Oncology, Yonsei Cancer Center, Severance Hospital, Yonsei University College of Medicine, Seoul, Korea; ${ }^{2}$ Center for Liver Cancer, Research Institute and Hospital, National Cancer Center, Goyang-Si, Korea; ${ }^{3}$ Department of Radiation
Oncology, Asan Medical Center, University of Ulsan College of Medicine, Seoul, Korea; ${ }^{4}$ Department of Radiation Oncology, Dongsan Medical Center, Keimyung University School of Medicine, Daegu, Korea; ${ }^{5}$ Department of Radiation Oncology, Dong-A University Hospital, Dong-A University School of Medicine, Busan, Korea; ${ }^{6}$ Department of Radiation Oncology, Chonnam National University Hospital, Gwang-Ju, Korea

Introduction: The purpose of this study was to investigate the efficacy and toxicity of radiotherapy (RT) following incomplete transarterial chemoembolization (TACE) in unresectable hepatocellular carcinoma (HCC).

Methods: The study was designed as a prospective phase II multicenter trial. Patients with unresectable HCC, who has viable tumor after TACE of no more than 3 courses, were eligible. Three dimensional-conformal RT (3D-CRT) was added for HCC with incomplete uptake of iodized oil, and the interval from TACE to RT was 4-6 weeks Prescribed total doses were up to 60 gray (Gy) in the absence of a no dose limitation to normal tissues near the RT site but were reduced by 46 Gy until normal tissue constraints were allowed. The 
primary endpoint of this study was the tumor response according to the modified Response Evaluation Criteria in Solid Tumors criteria after RT following incomplete TACE in unresectable HCC. Secondary endpoints were patterns of failure, progression-free survival (PFS), time to tumor progression (TTP), overall survival (OS) rates at 2 years, and treatment-associated toxicity. Survival was calculated from the start of RT using the Kaplan-Meier method. Any factor that influenced prognosis in univariate analyses were subjected to multivariate analyses using the Cox proportional hazards regression model.

Results: Between August 2008 and December 2010, 31 patients were enrolled. RT was delivered with a median total dose 54 Gy (range, 46-59.4 Gy) in 1.8 to 2 Gy per fraction. Most patients (29 patients, 93.5\%) received RT with a total dose $\geq$ $54 \mathrm{~Gy}$. A best objective in-field response rate was achieved in $83.9 \%$ of patients with complete response (CR) in $22.6 \%$ of patients and partial response (PR) in $61.3 \%$ of patients within 12 weeks post-RT. A best overall response rate was achieved in $64.5 \%$ of patients with CR in $19.4 \%$ of patients and PR in $45.1 \%$ of patients. The 2-year IFPFS, PFS, TTP, and OS rates were $45.2 \%, 29.0 \%, 36.6 \%$, and $61.3 \%$, respectively. The Barcelona-Clinic Liver Cancer stage was identified as significant independent prognostic factor for PFS ( $P=0.023$ ). Overall, 22 patients (59.1\%) had treatment failures. Hepatic in-field failure occurred in 10 patients (32.3\%), hepatic out-offield failure occurred in 11 patients (35.5\%), and extrahepatic failure (distant metastasis) occurred in 13 patients (41.9\%). The lung was the most common site for distant metastases. Classic radiation-induced liver disease was not observed. There was no treatment-related death or hepatic failure.

Conclusion(s): 3D-CRT following incomplete TACE achieves a promising objective response rate and is a safe and practical treatment option for patients with unresectable HCC.

\section{PS09-006 \\ Randomized Controlled Trial of Add-On Adriamycin for Hepatic Arterial Infusion Chemotherapy in Advanced Hepatocellular Carcinoma}

Myeong Jun Song ${ }^{1}$, Si Hyun Bae ${ }^{1 *}$, Jong Young Choi ${ }^{1}$, Seung Kew Yoon ${ }^{1}$, Jung Young Park ${ }^{2}$, Kwang Hyub Han', Sang Gyune Kim ${ }^{3}$, Young Sok Kim ${ }^{3}$, Yeon Seok Seo ${ }^{4}$, Hyung Joon Yim ${ }^{4}$, Soon Ho Um ${ }^{4}$, Woo Jin Chung, Jae Seok Hwang ${ }^{5}$, Sung-Bum Cho ${ }^{6}$, Jong Ryul Eun ${ }^{7}$, Heon Ju Lee ${ }^{7}$

${ }^{1}$ Internal Medicine, The Catholic University of Korea, Seoul, Korea; ${ }^{2}$ Internal Medicine, Yonsei University, Seoul, Korea; ${ }^{3}$ Internal Medicine, Soonchunghyang University, Bucheon, Korea; ${ }^{4}$ Internal Medicine, Korea University, Seoul, Korea; ${ }^{5}$ Internal Medicine, Keimyung University, Daegu, Korea; ${ }^{6}$ Internal Medicine, Chonnam National University, Gwanju, Korea; ${ }^{7}$ Internal Medicine, Yeungnam University, Daegu, Korea

Introduction: This multicenter, randomized, openlabeled, clinical trial evaluated the efficacy and safety of hepatic arterial infusion chemotherapy (HAIC) vs HAIC adding adriamycin in advanced HCC patients.

Methods: Fifty-six patients with advanced HCC were randomized to two treatment groups: (i) HAIC [ $\mathrm{n}=29,5$-fluorouracil (FU), $500 \mathrm{mg} / \mathrm{m} 2$ on days 1-3 and cisplatin, $60 \mathrm{mg} /$ $\mathrm{m} 2$ on day 2] and (ii) HAIC adding adriamycin [ $\mathrm{n}=27$, adriamycin, $50 \mathrm{mg} / \mathrm{m} 2$ on day 1, 5-fluorouracil (FU), $500 \mathrm{mg} / \mathrm{m} 2$ on days $1-3$ and cisplatin, $60 \mathrm{mg} / \mathrm{m} 2$ on day 2] every 4 weeks via an implantable port system. Primary efficacy endpoint was overall survival (OS). Treatment response and time to progression (TTP) were secondary endpoints.

Results: Treatment response rates did not differ significantly between the two treatment groups (partial response: 17.2 vs. $7.4 \%$, stable disease: 48.2 vs. $66.6 \%$ and progressive disease: 34.4 vs. $25.9 \%$, respectively). Time to progression (5.4 vs. 5.8 months, $\mathrm{P}=0.863$ ) and overall survival (OS) (11.1 vs. 8.8 months, $\mathrm{P}=0.448$ ) were not significantly different. When the factors affecting patient OS were analyzed, disease control rate [P < 0.001, HR 6.437 (95\% CI 2.580-16.064)] was independently associated with OS. Age ( $\geq 60$ years) and serum AFP level ( $\geq 200 \mathrm{ng} / \mathrm{dL}$ ) also were significant factors for OS $[\mathrm{P}=0.007, \mathrm{HR} 4.945$ (95\% CI 1.543-15.850), $\mathrm{P}=0.048, \mathrm{HR}$ 2.677 (95\% CI 1.010-7.095), respectively]. Grade 4 treatment related toxicity and mortality was not observed in both groups.

Conclusion(s): Although both HAIC regimens are safe and effective in patients with advanced HCC, HAIC adding adriamycin did not show delayed tumor progression and survival benefit compared to HAIC in advanced HCC. 


\section{Category 10 \\ Novel Approach and Innovative Trials}

\section{PS10-001 \\ Validation of APRI and FIB-4 for Liver Fibrosis Assessment in $1660 \mathrm{HBV}$-Related Hepatocellular Carcinoma after Hepatectomy Guangqin Xiao, Lunan Yan*, Jiayin Yang \\ Department of Liver Surgery, West China Hospital of Sichuan University, Chengdu, China}

Introduction: Non-invasive methods have been developed as alternatives to liver biopsy to assess liver fibrosis. This study examined the validity of the aspartate aminotransferase-to-platelet ratio index (APRI) and the fibrosis index based on four factors (FIB-4) for the assessment of liver fibrosis in hepatitis B virus (HBV)-related hepatocellular carcinoma (HCC) patients after hepatectomy.

Methods: We enrolled HBV-positive patients with liver cancer after liver resection. We investigated the relationship between two non-invasive methods, APRI and FIB-4, in different stages of liver fibrosis using the chi-squared $\left(\chi^{2}\right)$ test and Spearman's rank correlation test. Weverified the accuracy, efficacy and reliability of APRI and FIB-4 for liver fibrosis assessment based on their sensitivity, specificity, diagnostic efficiency, positive predictive value (PPV), negative predictive value (NPV), kappa ( $\kappa$ ) value and area under the receiveroperating characteristic curve (AUC).

Results: A total of 1,660 HBV-related adult HCC patients were included in our study. APRI (rs = 0.266) and FIB-4 (rs=
0.239 ) were positively correlated with liver fibrosis in HBVrelated HCC patients, and $\chi^{2}$ analysis demonstrated that APRI and FIB-4 correlated with liver cirrhosis (both $\mathrm{P}$ values $<0.001)$. The respective AUC values for APRI and FIB-4 were 0.650 and 0.595 for the differentiation of significant fibrosis, 0.688 and 0.656 for the differentiation of advanced fibrosis and 0.680 and 0.657 for the differentiation of cirrhosis.

Conclusion(s): The APRI and FIB-4 models are suitable for the staging of liver fibrosis in adult HBV-related HCC patients. However, the validity of APRI and FIB-4 for the assessment of liver fibrosis is not satisfactory.

\section{PS10-002 \\ Survival Outcomes of Patients with Advanced Unresectable Hepatocellular Carcinoma in Relation to External Radiotherapy \\ Yu-Min Lin*, Chao-Sheng Liao, Kuo-Ching Yang \\ Gastroenterology, Shin Kong Wu Ho-Su Memorial Hospital, Taipei, Taiwan}

Introduction: Radiotherapy (RT) and target therapy have made great progress for cancer treatments recently. We aimed to understand the outcome of external RT in combination with target therapy (RT+target) for the patients with advanced unresectable hepatocellular carcinoma (HCC).

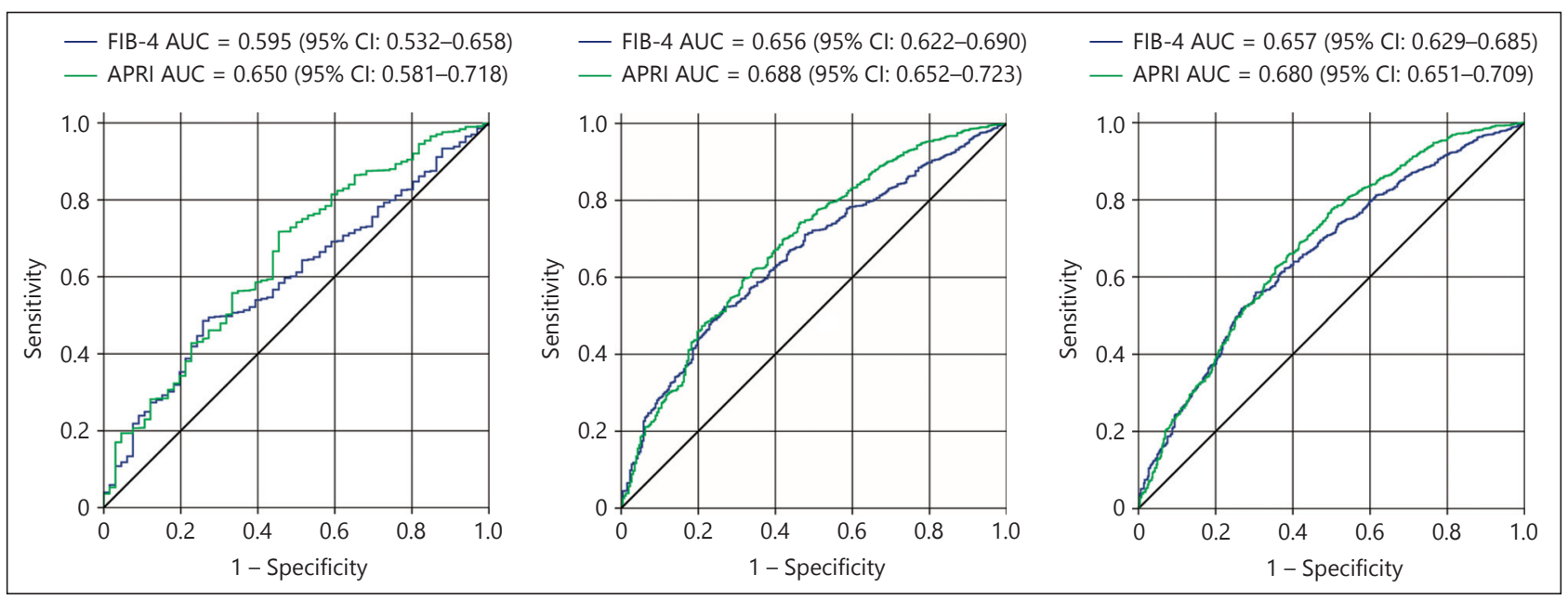

Fig. 1. ROC plot for the fibrosis index based on four factors (FIB-4) and aspartate aminotransferase (AST)-to-platelet ratio index (APRI) in the differentiation of different stages of fibrosis: (A) ROC plot for FIB-4 and APRI in the differentiation of significant fibrosis ( $\geqq S 3$ ); (B) ROC plot for FIB-4 and APRI in the differentiation of advanced fibrosis ( $\geqq S 4$ ); (C) ROC plot for FIB-4 and APRI in the differentiation of cirrhosis ( $\geqq$ S5) (for abstract PS10-001). 


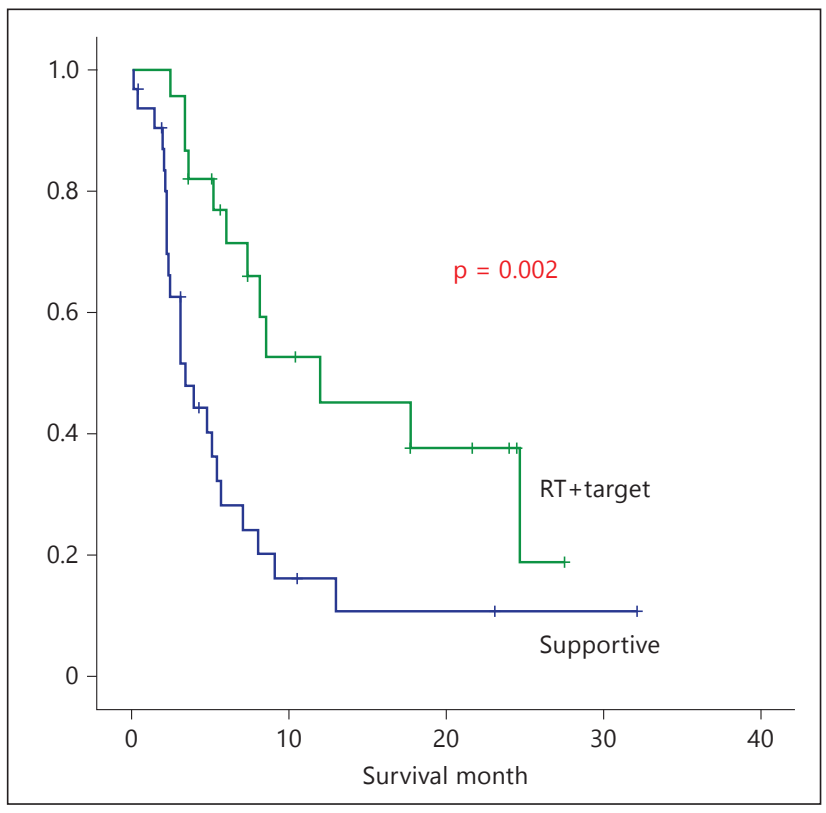

Fig. 1. Survival of advanced HCC with the treatment of RT+target (for abstract PS10-002).

Methods: We retrospectively analyzed 423 patients with HCC registered in the cancer database of Shin-Kong hospital between January 2008 and December 2011. The stage of HCC was determined according to BCLC system. The mean age is $63.3 \pm 12.6$ years. Male to female ratio is $304: 119$. The numbers of BCLC stage A, B, C and D were 183, 60, 129, and 51 respectively. Among 129 patients with BCLC stage $C, 116$ were unresectable and were enrolled for subsequent analysis. The main outcome measures were overall survival rates in relation to treatment modalities. Statistical methods included Kaplan-Meier analysis and log-rank test. A p value $<0.05$ was considered as significant.

Results: The median survival time of these 116 unresectable advanced HCC patients was 6.6 months $(95 \% \mathrm{CI}$, 4.6-8.7). Treatment modalities for 116 were divided as group I: TACE alone ( $n=23)$, group II: with RT component $(n=61)$ and group III: others $(n=32)$. The median survival time for group I, II and III were 9.9 months (95\% CI, 1.2-18.7), 7.4 months (95\% CI, 5.1-9.4) and 3.5 months (95\% CI, 2.4-4.5) respectively $(\mathrm{p}<0.001)$.

Subsequent survival analysis for group II was performed. The median survival time of patients treated with RT+target $(\mathrm{n}=23)$, TACE+RT $(\mathrm{n}=10), \mathrm{RT}+$ chemotherapy $(\mathrm{n}=18)$, and RT alone $(\mathrm{n}=10)$ were 12.0 months (95\% CI, 1.3-22.8), 6.1 months (95\% CI, 3.2-9.0), 5.7 months (95\% CI, 2.7-8.6), and 3.6 months (95\% CI, 1.6-5.7) respectively ( $\mathrm{p}<0.001)$.

Conclusion(s): Our results suggest RT+target therapy for patients with advanced unresectable HCCs could reach a better survival outcome in comparison with other treatment modalities. Further prospective studies comparing RT+target therapy with sorafenib are needed to understand the survival benefits of this treatment combination.

\section{PS10-004 \\ Efficacy and Safety of Nintedanib vs Sorafenib in Asian Patients with Advanced Hepatocellular Carcinoma: A Randomised Phase II Trial}

Chia-Jui Yen $^{1 *}$, Tae-You Kim², Yin-Hsun Feng ${ }^{3}$, Yee Chao ${ }^{4}$, Deng-Yn Lin ${ }^{5}$, Arsene-Bienvenu Loembe ${ }^{6}$, Julia Hocke ${ }^{7}$, Dennis Chin-Lun Huang ${ }^{8}$, Baek-Yeol Ryoo 9 , Ann-Lii Cheng ${ }^{10}$

${ }^{1}$ Internal Medicine, Division of Hematology and Oncology, National Cheng Kung University Hospital, Tainan, Taiwan; ${ }^{2}$ Internal Medicine, Seoul National University Hospital, Seoul, Korea; ${ }^{3}$ Internal Medicine, Division of Hematology and Oncology, Chi Mei Medical Centre Yongkang, Tainan City, Taiwan; ${ }^{4}$ Cancer Center, Division of Chemoradiotherapy, Taipei Veterans General Hospital, Taipei City, Taiwan; ${ }^{5}$ Gastroenterology and Hepatology, Chang Gung Memorial Hospital; Chang Gung University, College of Medicine, Taoyuan, Taiwan; ${ }^{6}$ Medical, Boehringer Ingelheim B.V., Alkmaar, Netherlands; ${ }^{7}$ Global Biometry and Clinical Applications, Boehringer Ingelheim Pharma GmbH and Co. KG, Biberach, Germany; ${ }^{8}$ Medical, Boehringer Ingelheim Taiwan Ltd., Taipei, Taiwan; ${ }^{9}$ Oncology, Asan Medical Centre, Seoul, Korea; ${ }^{10}$ Medical Oncology, National Taiwan University Hospital, Taipei City, Taiwan

Introduction: Nintedanib is an oral angiokinase inhibitor of vascular endothelial growth factor receptors, platelet derived growth factor receptors, and fibroblast growth factor receptors. This randomised, multicentre, open-label, Phase I/ II study (NCT00987935) evaluated efficacy and safety of nintedanib versus sorafenib in Asian patients with advanced hepatocellular carcinoma (HCC).

Methods: Treatment-naïve patients with advanced HCC unamenable to curative/locoregional therapy, Child-Pugh score 5-6, Eastern Cooperative Oncology Group-Performance Status $\leq 2$, and aspartate aminotransferase (AST)/alanine aminotransferase $\leq 2 \times$ upper limits of normal were enrolled. Patients were randomised 2:1 to nintedanib $200 \mathrm{mg}$ bid or sorafenib $400 \mathrm{mg}$ bid continuously in 28-day cycles. Treatment beyond disease progression (PD) was allowed. Primary endpoint was time to progression (TTP) by central review (RECIST 1.0). Secondary end points included objective tumour response (OR) by central review, overall survival (OS), and incidence and intensity of adverse events (AEs). All analyses were descriptive and exploratory.

Results: Ninety-five eligible patients received nintedanib $(n=63)$ or sorafenib $(n=32)$. Arms were balanced except for macrovascular invasion (nintedanib $48 \%$ vs sorafenib $28 \%$ ). By September 28, 2012, 86 patients had discontinued the study due to PD $(n=67)$, AEs $(n=13)$, or other reasons $(n=$ 6). Median time to discontinuation of nintedanib or sorafenib was 3.0 and 3.8 months, respectively (HR 1.19 [95\% CI: 0.761.88]). TTP was not different between the nintedanib and 
sorafenib arms (median, 2.7 [95\% CI: 1.8-3.7] vs 3.7 [95\% CI: 2.6-7.3] months; HR 1.36 [95\% CI: 0.80-2.30]), as was OR rate (3.2\% vs 3.1\%) and OS (median, 10.7 [95\% CI: 8.1-14.8] vs 9.5 [95\% CI: 7.4-11.0] months; HR 0.77 [95\% CI: 0.42-1.41]). Common Terminology Criteria for AEs Grade 3 (32\% vs 56\%) and Grade 4 ( $8 \%$ vs $16 \%$ ) were less frequent with nintedanib than sorafenib. Any-grade (nintedanib vs sorafenib) diarrhoea, abdominal pain, abdominal pain upper, hand-foot skin reaction, AST increases, rash and alopecia were more common with sorafenib; decreased appetite, nausea, vomiting, abdominal distension, ascites and back pain were more common with nintedanib. Eleven patients $(18 \%)$ in the nintedanib arm and 18 (56\%) in the sorafenib arm had dose reductions due to AEs.

Conclusion(s): Nintedanib appears active and shows similar efficacy to sorafenib, with a more favourable and manageable AE profile in Asian patients with advanced HCC. Further studies of nintedanib in HCC are warranted.

\section{PS10-005 \\ Clinical Activity of G-202, A Thapsigargin- Based Prostate-Specific Membrane Antigen (PSMA)-Activated Prodrug, in Patients with Progressive Hepatocellular Carcinoma (HCC) \\ Devalingam Mahalingam $^{1 *}$, John Nemunaitis², John Sarantopoulos ${ }^{1}$, Victoria Allgood ${ }^{3}$, Michael Kurman ${ }^{4}$, Luis Campos ${ }^{5}$ \\ ${ }^{1}$ Medicine, Institute for Drug Development, Cancer Therapy and Research Center at University of Texas Health Science Center San Antonio, San Antonio, United States; ${ }^{2}$ Medicine, Mary Crowley Cancer Research Center, Dallas, United States; ${ }^{3}$ GenSpera, Inc, San Antonio, United States; ${ }^{4}$ GenSpera, Inc, San Antonio, United States; ${ }^{5}$ Medicine, 4Oncology Consultants, PA, Houston, United States}

Introduction: Thapsigargin induces apoptosis through disruption of calcium homeostasis. G-202 is a thapsigarginbased prodrug whose cytotoxic activity is blocked by a masking peptide that is cleaved by PSMA, a membrane-bound protease expressed in prostate cancer cells and the endothelium of tumor vasculature but not in most other tissues or the vasculature of normal tissue. In a Phase I study of G-202 in patients with advanced solid tumors, prolonged disease stabilization was observed in the subset of patients with hepatocellular carcinoma and prompted development of a Phase II study to further evaluate activity of G-202 in this patient population.

Methods: G-202 was administered by intravenous infusion on Days 1, 2 and 3 of a 28-day cycle. Five patients were treated in the Phase I study at $40 \mathrm{mg} / \mathrm{m} 2$ on Day 1 and $66.8 \mathrm{mg} / \mathrm{m} 2$ on Days 2 and 3 (40/66.8/66.8). Sixteen patients have been treated in the Phase II study, which remains open to enrollment. Among the 16, 6 were treated at 40/66.8/66.8, but this regimen was not well-tolerated and it was modified to $40 \mathrm{mg} / \mathrm{m} 2$ on Days 1,2 and $3(40 / 40 / 40)$. All patients were ECOG 0-1, Child-Pugh A5, A6 or B7 and had progressed on sorafenib.

Results: Among the combined 21 patients with HCC treated to date in the Phase I and Phase II studies, G-202 related AEs of any grade in at least $3(14 \%)$ patients were ALT increased ( $6 \mathrm{pts}$ ), anorexia (5 pts), bilirubin increased (5 pts), chills (4 pts), creatinine increased ( 8 pts), fatigue (8 pts), hiccups (5 pts), hyperkalemia (4 pts), nausea (8 pts), rash/ pruritis (13 pts). G-202 related AEs $\geq$ Grade 2 in at least 2 (11\%) patients were albumin decreased ( 2 pts), ALT increased (4 pts), bilirubin increased (4 pts), BUN increased (2 pts), creatinine increased ( $5 \mathrm{pts})$, diarrhea (2 pts), fatigue (5 pts), hyperglycemia (3 pts), hyperkalemia (4 pts), LDH increased (2 pts), nausea (2 pts), rash/pruritis (2 pts), thrombocytopenia ( 2 pts). Due to creatinine elevations, sometimes reported as acute kidney injury or acute renal failure, prophylactic intravenous hydration on each day of infusion is administered to ameliorate the incidence and severity of creatinine elevations. Related SAEs in this patient population have been creatinine increase/acute renal failure/acute kidney injury (3 pts) and congestive heart failure (1 pt). Among these 21 patients, 77 cycles of G-202 have been administered (average 4.4 cycles, range 1-12), including 41 cycles at $40 / 40 / 40$ and 36 cycles at 40/66.8/66.8. To date, 18 patients are evaluable for response; while objective responses (CR, PR) have not been observed in these patients with advanced disease, the rate of disease stabilization has been remarkable, with 13 of 18 patients patients with HCC (72\%) exhibiting SD. 9 pts (50\%) had SD $\geq 4$ months; 5 pts (28\%) had SD $\geq 6$ months with some patients still on study. One patient completed 9 cycles before experiencing a dose delay due to an unrelated AE; he was removed from the study but has received 7 additional cycles (total 16 cycles over 20 months) and remains in SD under a compassionate use protocol.

Conclusion(s): G-202 administered intravenously for 3 consecutive days of a 28-day cycle is generally well-tolerated and promotes disease stabilization in patients with advanced HCC who have progressed on sorafenib. HCC lesions are typically highly vascularized and exploratory studies to evaluate possible effects of G-202 on tumor vasculature have been incorporated into the Phase II study. 


\section{PS10-006 \\ Results from a Phase 2 Study Support a Phase 3 Trial of Cabozantinib vs Placebo in Hepatocellular Carcinoma Patients Post-Sorafenib (Celestial; NCT01908426)}

Ghassan K. Abou-Alfa ${ }^{1 *}$, Ann-Lii Cheng ${ }^{2}$, Tim Meyer ${ }^{3}$, Anthony B. El-Khoueiry', Yihua Lee ${ }^{5}$, Anne E. Borgman-Hagey ${ }^{6}$, Robin Kate Kelley

${ }^{1}$ Medicine, Memorial Sloan-Kettering Cancer Center and Weill Cornell Medical College, New York, United States; ${ }^{2}$ Oncology, National Taiwan University Hospital, Taipei, Taiwan; ${ }^{3}$ Medical Oncology, Royal Free Hospital, London, United Kingdom; ${ }^{4}$ Medicine, University of Southern California, Keck School of Medicine, Los Angeles, United States; ${ }^{5}$ Biostatistics, Exelixis Inc., South San Francisco, United States; ${ }^{6}$ Clinical Development, Exelixis Inc., South San Francisco, United States; ${ }^{7}$ Medicine, UCSF Helen Diller Family Comprehensive Cancer Center, San Francisco, United States

Introduction: Currently, no systemic therapies are approved for patients (pts) with hepatocellular carcinoma (HCC) who progress following sorafenib (SOR). Cabozantinib (Cabo) is an oral inhibitor of tyrosine kinases including MET, RET and VEGFRs. MET and VEGFR signaling have been implicated in tumor neo-angiogenesis and invasion. MET is overexpressed in many HCC tumors compared with non-tumor liver tissue. Higher MET expression is linked to poor prognosis and may mediate escape from VEGFR inhibition. Thus, simultaneous inhibition of MET and VEGFRs in HCC is a rational approach for clinical investigation.

Methods: A cohort of pts with advanced HCC was treated with cabo in a phase 2 randomized discontinuation study (XL184-203). Pts had measurable disease per RECIST, $\leq 1$ prior systemic regimen and Child-Pugh score of A. Pts received cabo at $100 \mathrm{mg}$ qd over a $12 \mathrm{wk}$ Lead-In stage. Tumor response (protocol-specified RECIST) was assessed q6 wks. Treatment $\geq$ wk 12 was based on response: pts with partial response (PR) continued open-label cabo, pts with stable disease (SD) were randomized to cabo vs placebo, and pts with progression discontinued. The primary endpoint was response rate (ORR) per RECIST in the Lead-In stage, and progression-free survival (PFS) in the Randomized stage.

Results: XL184-203 HCC pts' ( $\mathrm{N}=41)$ characteristics were: male 76\%; Asian 37\%; HBV/HCV 24\%/22\%; extrahepatic spread $73 \%$; prior systemic therapy $80 \%$ (prior SOR in 51\%). $2 / 37$ pts evaluable for tumor assessment at $12 \mathrm{wks}$ had a confirmed PR (CPR) by RECIST (RR 5\%). In addition, one pt randomized to placebo at Week 12 achieved a cPR at 18 weeks. $28 / 36$ pts $(78 \%)$ with $\geq 1$ post-baseline scan had tumor regression. The overall disease control rate (PR+SD) at Wk 12 was $66 \%$ (Asian subgroup: 73\%). Median PFS was 4.4 mos overall from Study Day 1, and 5.2 months if prior SOR. AFP responses (decrease of $>50 \%$ in pts with baseline elevated
AFP and $\geq 1$ post-baseline result): $10 / 26$ (38\%). Most common Gr 3/4 AEs: diarrhea (20\%), palmar-plantar erythrodysesthesia (15\%), and thrombocytopenia (15\%).

Conclusion(s): Cabo treatment exhibited activity in HCC in XL184-203 with a safety profile comparable to other VEGFR tyrosine kinase inhibitors. These results provided the rationale for development of the recently initiated CELESTIAL trial.

CELESTIAL is a phase 3, randomized, double-blind study that evaluates the efficacy and safety of cabo compared with placebo in pts with advanced HCC previously treated with SOR who have progressed following 1-2 prior systemic treatments. Pts must be $\geq 18$ year old, Child-Pugh Score of A, and ECOG PS $\leq 1$. Pts are randomized 2:1 to receive either cabo (60 $\mathrm{mg} \mathrm{qd}$ ) or placebo. Stratification factors are etiology of disease, geographic region and the presence of extrahepatic spread of disease and/or macrovascular invasion. The primary endpoint is OS. Secondary endpoints are PFS and ORR by RECIST 1.1 . Additional endpoints include safety, tolerability, circulating tumor cells, serum bone and plasma biomarkers, effects on bony disease assessed by bone scan and health-related quality of life. Enrollment was initiated in September 2013. Target recruitment is 760 pts. A total of 621 events would provide $90 \%$ power to detect a $31.6 \%$ increase in OS (HR $=0.76)$. This global study is ongoing in 14 countries.

\section{PS10-008 \\ NF-kB Mediated CD47 Upregulation Promotes Sorafenib Resistance and Its Therapeutic Implications in Hepatocellular Carcinoma}

Jessica Lo, Terence Kin-Wah Lee*, Irene Oi-Lin Ng

Pathology, State Key Laboratory for Liver Research, The University of Hong Kong, Hong Kong

Introduction: Hepatocellular carcinoma (HCC) is the third leading cause of cancer-related death worldwide. It often diagnosed at an advanced stage where surgical resection is not possible. Currently, sorafenib is the new standard treatment used to treat patients with advanced HCC. Unfortunately, the survival benefit of sorafenib is modest with a median survival of 3 months due to treatment resistance hence a better understanding of the resistance mechanism is urgently needed. Accumulating evidence suggests that tumor contains a subpopulation of cancer stem cells (CSCs) capable of treatment resistance. Elimination of this subpopulation is vital in tackling treatment resistance. Previously, our group identified CD47 as a novel therapeutic target against liver CSCs, which suggests a possibility of targeting CD47 to eliminate the CSCs thereby evading sorafenib resistance. This, together with bioinformatics data showing two putative $\mathrm{NF}-\mathrm{KB}$ binding sites on CD47 promoter, has promoted us to investigate the involvement of NF- $\mathrm{KB}$ in CD47 expression and 


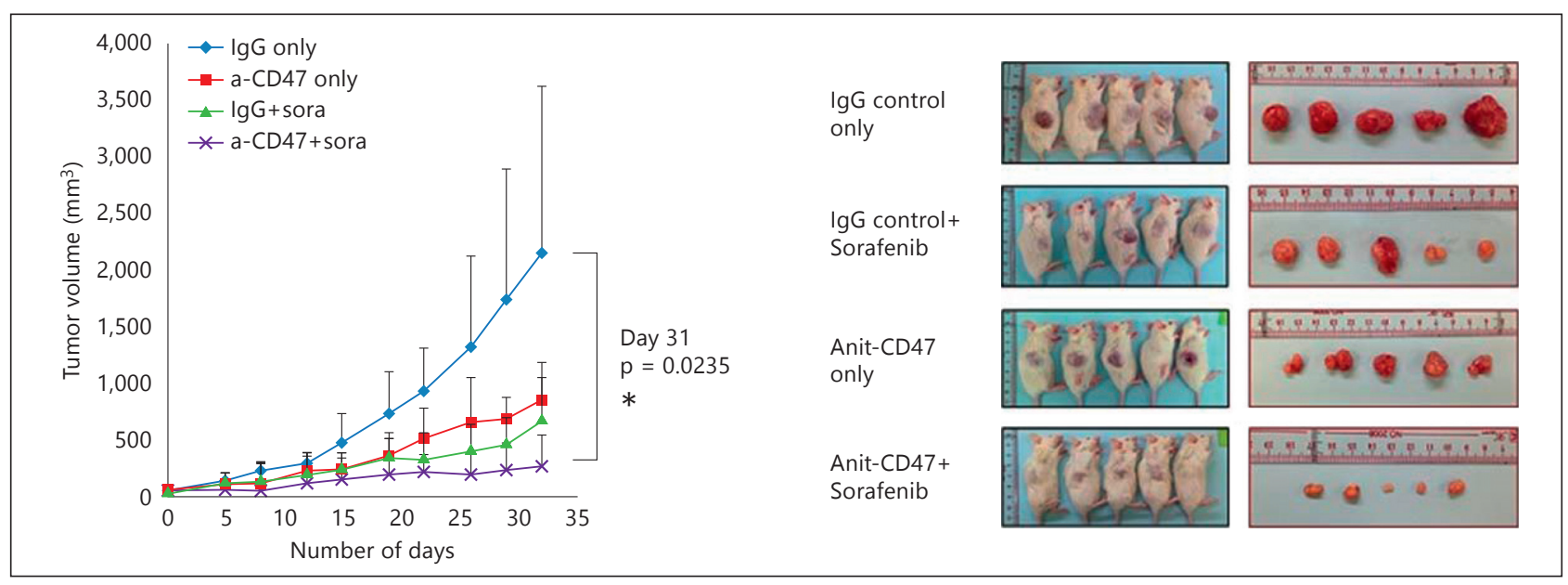

Fig. 1. In vivo anti-CD47 antibody and sorafenib cotreatment experiment using patient derived xenograft mouse model (for abstract PS10-008).

the suitability of CD47 as a novel therapeutic target for combination therapy with sorafenib in HCC.

Methods: Sorafenib-resistant clones were developed both in vitro and in vivo through continuous exposure to Sorafenib. CD47 and NF- $\mathrm{KB}$ expression of the resistant clones were detected by flow cytometry and western blot analyses respectively. To investigate the direct interaction between $\mathrm{NF}-\kappa \mathrm{B}$ and CD47, inhibitor and stimulator of NF- $\kappa \mathrm{B}$ (IMD0354 and TNF- $\alpha$ ) was used to examine the effect of NF- $\kappa B$ alternation on CD47 expression. In addition, ChIP promoter binding assay was employed to examine NF- $\kappa B$ binding on the putative sites. Stable knockdown approach was used to examine the role of CD47 in sorafenib sensitivity. The therapeutic role of targeting the CD47 alone or in combination with sorafenib was evaluated both in vitro and in vivo.

Results: Sorafenib-resistant clones were successfully developed in vitro in Huh7 and BEL7402 cell lines and in vivo using patient-derived xenografts PDTX\#1 and PDTX\#5. We found that sorafenib-resistant clones showed enhanced CSC properties, which is also accompanied with increase in CD47 expression. Consistent to the bioinformatics analysis, activation of NF- $\mathrm{KB}$ was also observed. The expression of CD47 was found to be regulated by NF- $\mathrm{KB}$ through a specific responsive element in the CD47 promoter as assessed by ChIP promoter binding assay. Further evidence of NF- $\mathrm{B}$ regulation of CD47 was shown by treatment with NF- $\kappa$ B inhibitor (IMD0354 ) and stimulator TNF- $\alpha$. These results suggested that NF- $\kappa B$ mediated CD47 upregulation promotes sorafenib resistance in HCC. Suppression of CD47 expression in MHCC97L and Huh7 cells by lentiviral based knockdown approach showed increased sensitivity to sorafenib. Using patientderived xenograft (PDTX\#5), we examined the effect of sorafenib (100 mg/kg/day) and its combined effect with antiCD47 antibody (500 $\mu \mathrm{g} / \mathrm{mice} /$ day) when the tumor reached approximately $4 \times 4 \mathrm{~mm}$. After 31 days of treatment, we found sorafenib and anti-CD47 antibody alone suppressed the tumor growth with similar effects by approximately 2.5 and 3 -folds respectively. The combination of sorafenib and anti-CD47 antibody exhibited a maximal effect on tumor suppression by approximately 8-folds (Refer to figure).

Conclusion(s): NF- $\kappa \mathrm{B}-$ mediated CD47 upregulation may contribute to sorafenib resistance and combination of CD47 antibody and sorafenib is a potential novel therapeutic regimen against HCC.

\section{PS10-010 \\ Efficacy and Safety of Stereotactic Body Radiation Therapy for Hepatocellular Carcinoma Refractory or Unsuitable for Other Therapeutic Modalities}

Sang Youn Hwang ${ }^{1 *}$, Jung Woo $\mathrm{Im}^{1}$, Seon-Mi Lee ${ }^{1}$, Joon Suk Kim ${ }^{1}$, Sang Bu Ahn', Eun Kyeong ${ }^{3}$, Cheol Won ${ }^{4}$, Gwang $\mathrm{Mo}^{5}$

${ }^{1}$ Internal Medicine, Dongnam Institute of Radiological \& Medical Sciences, Busan, Korea; ${ }^{2}$ Radiology, Dongnam Institute of Radiological \& Medical Sciences, Busan, Korea; ${ }^{3}$ Radiology, Ji, Busan, Korea; ${ }^{4}$ Radiation Oncology, Choi, Busan, Korea; ${ }^{5}$ Radiation Oncology, Yang, Busan, Korea

Introduction: The aim of this study was to evaluate efficacy and safety of stereotactic body radiotherapy (SBRT) for the naïve or salvage treatment of inoperable hepatocellular carcinoma (HCC) were unsuitable for other therapeutic options

Methods: The authors reviewed the medical records of 49 patients that were treated by SBRT in our institution when they had HCC without another standard treatment option or complete response of loco-regional therapy between August 
2010 and February 2014. All patients SBRT dosages (24-60 Gy from two to five fractions) were administered according to tumor volume. Survival, response, and toxicities were evaluated. Response evaluation was performed according to modified Response Evaluation Criteria for Solid Tumors.

Results: Forty-three patients had Child-Pugh class A disease, 6 patients had class B disease. Twenty patients had macrovascular invasion (12 portal vein thrombosis, 4 hepatic vein thrombosis, 4 both venous thrombosis), 2 patients had bile duct invasion. The median greatest tumor dimenstion was $32 \mathrm{~mm}$ (range, 10-170 mm). The median overall survival was 10 months (range 2-43 months) and the median progressionfree survival was 7 months (range 1-42 months). Thirteenseven patients $(75.5 \%)$ achieved complete response within 6 months after complete SBRT, 4 patients $(8.1 \%)$ had a partial response, 7 patients $(14.3 \%)$ had stable disease, and 1 (2.1\%) patients had progression disease. Infield local recurrence was observed in 4 patients (8.1\%), and outfield intrahepatic failure was 20 patients $(40.8 \%)$ and extrahepatic metastasis was 15 patients $(30.6 \%)$. Three patients $(10 \%)$ experienced grade 3 gastrointestinal toxicity, 1 patients $(3.3 \%)$ experienced grade 4 gastric ulcer perforation, and 4 patients (13.3\%) experienced mild pneumonitis and 1 patients $(2.1 \%)$ experienced burn requiring skin graft.

Conclusion(s): Our study suggests that SBRT can be effective and safe modality that achieves promising rates of local control and survival in HCC refractory or unsuitable for other therapeutic options, even with vascular or bile duct invasion. A further well controlled, large scaled study to reduce gastrointestinal and pulmonary toxicity is recommended.

\section{PS10-011 \\ Combined Targeting of mTORC1 and mTORC2 Synergistically Inhibits Proliferation of Hepatocellular Carcinoma Cells: Effects of Everolimus and Ku0063794}

\author{
Say-June Kim* \\ Department of Surgery, the Catholic University of \\ Korea, Daejeon St. Mary's Hospital, Daejeon, Korea
}

Introduction: The mTOR has recently generated great interest in cancer treatment. Rapamycin, which is both an immunosuppressant regimen and an effective inhibitor of mTOR as well, showed growth inhibitory and anti-angiogenic properties in certain gastrointestinal malignancies in experimental models. Furthermore, it was also reported that de novo rapamycin-based immunosuppression in patients who had received liver transplantation due to hepatocellular carcinoma was associated with the satisfactory oncologic outcome, even in selected patients beyond Milan criteria. However, rapamycin only exerts the inhibitory effects to mTORC1, its effects should be compared with the effects exerted by a complete mTORC1/2 blocker.

Methods: Everolimus (Rapamycin analogue; mTORC1 inhibitor) and $\mathrm{Ku}-0063794$ (mTORC1/2 inhibitor) was treated in HepG2 cell line respectively and with the combination. Ez-syntax assay, FACS, Autophagy assay, and Western blot were implemented.

Results: Increase in cell proliferation was pronounced in combined treatment with everolimus and Ku-0063794

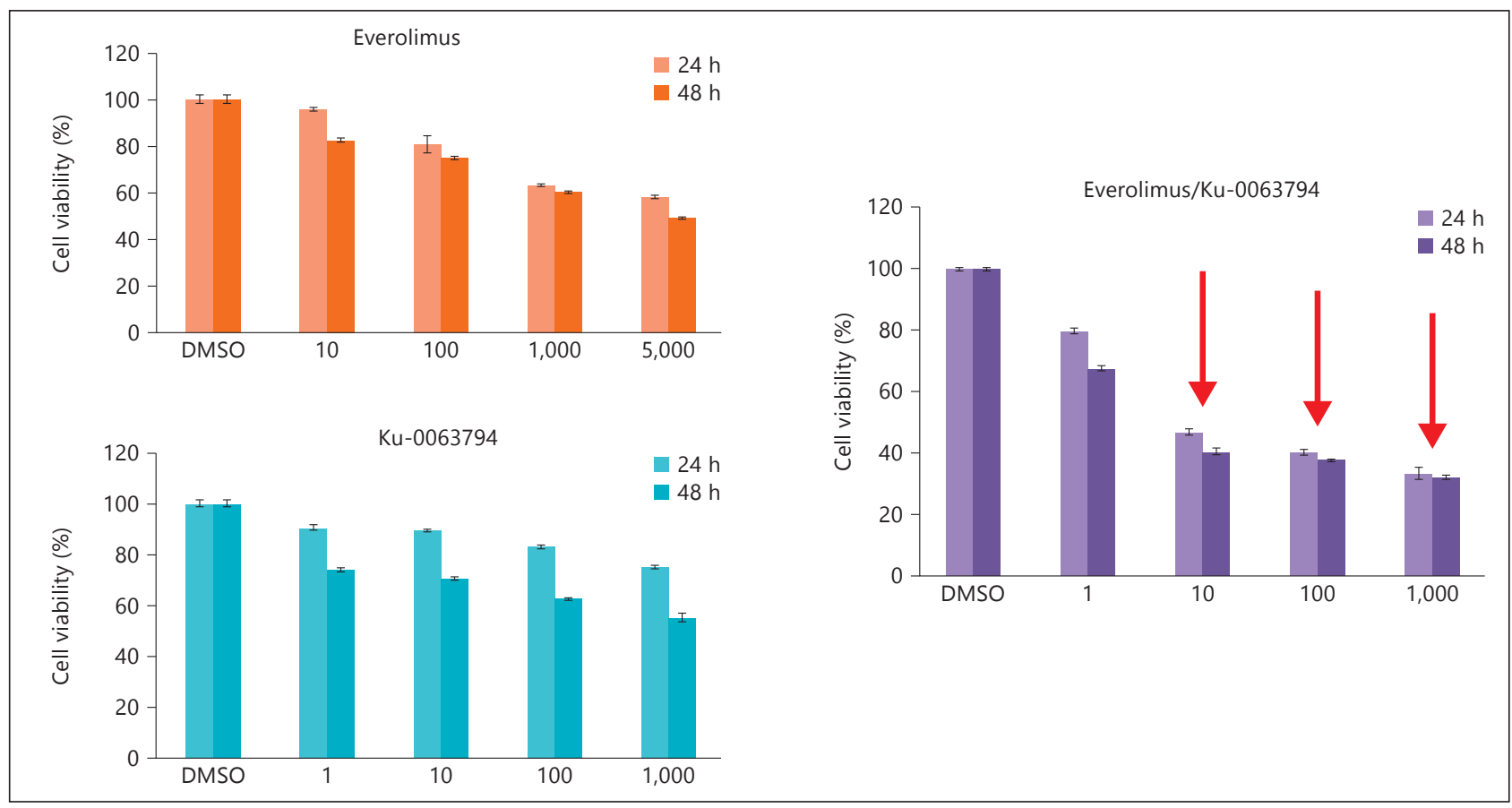

Fig. 1. (for abstract PS10-011). 
compared with either drug alone. The proportion of cells arrested in G1 and G0 phase was increased in the combination group. In the combination group, the cell death was demonstrated in the form of autophagy rather than apoptosis. Further, the combination could noticeably suppress tumor cell invasion and the phosphorylation of AKT, p70S6K and STAT3.

Conclusion(s): The combination of everolimus and $\mathrm{Ku}-0063794$ effectively suppressed the tumor biology in terms of survival, cell cycling, invasion, and related signal transduction. Theoretically, solitary inhibition of mTORC1 can release the inhibition of PI3 kinase, which activates AKT, favoring tumor growth. Therefore, balanced blockage of mTORC1 and mTORC1 should be taken into consideration to effectively enhance anti-cancer effects.

\section{PS10-012 \\ Intermittent Microwave Energy Radiation Create a Larger Ablation Zone with Lower Tissue Temperature \\ Massatoshi Tanaka $^{1 *}$, Yoshinobu Kohara² \\ ${ }^{1}$ Hepatology, Yokokura Hospital, Miyama, Japan; \\ ${ }^{2}$ Research Labo., Alfresa-Pharma. Co. Ltd, Osaka, Japan}

Introduction: The ablation effect of microwave (MW) energy (molecular dipole rotation theory) differs fundamentally from that of radiofrequency (RF) energy (electric currency theory), and microwave ablation (MWA) may expand the role of tumor ablation by providing a more homogenous and predictable ablation zone. However, the MW energy radiation make a higher tissue temperature than RF energy, and it may lead a serious complication sometimes with charred tissue around MW antenna. Herein, we report experimental results of a new MWA protocol using ex-vivo cattle liver.

Methods: Microtaze AZM-550 (Alfresa, Okaka, Japan) creating $2.45 \mathrm{GHz}$ MW energy was used, and MW antenna used was $2 \mathrm{~mm}$ in size. While Japanese conventional protocol was $80 \mathrm{~W}$ and $1 \mathrm{~min}$. continuous radiation, our new protocol was $80 \mathrm{~W}$, and $10 \mathrm{sec}$. radiation and $5 \mathrm{sec}$. rest as one cycle, and this cycle repeated 20 to 30 times. Using ex-vivo cattle liver in water bath maintaining 250, single MWA session was conducted with single MW antenna comparing different protocol, and ablation area was measured, respectively. Standing Wave Ratio and tissue temperature around antenna during MWA were also monitored.

Results: While a conventional MWA protocol created a $15 \mathrm{~mm}$ width, our new protocol created a $25 \mathrm{~mm}$ width by 20 cycles (5 min.) and $30 \mathrm{~mm}$ width by 30 cycles $(7.5 \mathrm{~min}$.), respectively. SWR and tissue temperature of a new protocol were also lower than a conventional one. With triplet antenna setting $2 \mathrm{~cm}$ distance each created over $5.5 \mathrm{~cm}$ width ablation zone by $80 \mathrm{~W}, 30$ cycle MWA.
Conclusion(s): Our new protocol of MWA product a wider ablation zone with an effective radiation energy (lower SWR) and lower tissue temperature around antenna than conventional protocol currently applied in Japan. Our protocol for MW energy radiation may be useful for liver tumor ablation.

\section{PS10-013 \\ Proton-Beam Radiotherapy Is an Effective Alternative Therapy for Locally Unresectable Hepatocellular Carcinoma Not-Feasible Curative Treatment \\ Masatoshi Tanaka* \\ Hepatology, Yokokura Hospital, Miyama, Japan}

Introduction: This phase II trial was conducted to determine the efficacy and toxicity of proton beam radiotherapy for patients with locally unresectable hepatocellular carcinoma.

Methods: Cirrhotic patients were eligible if they had a Child-Pugh score of 7 or less. Eligible patients included single or localized multiple hepatocellular carcinoma, sized less than $5 \mathrm{~cm}$ with in BCLC-A stage. Patients with lymph node or distant metastases were ineligible. Daily proton beam radiotherapy was directed to the liver tumor with an additional 1-2 cm margin. The total dose was 66 cobalt Gray equivalents, administered in 10 divided fractions within 2 weeks.

Results: Fourteen patients have completed treatment and have been followed up for a minimum of 3 months, with a median follow-up period of 14 months. The mean age was 70 years, and average tumor size was $2.8 \mathrm{~cm}$. Post-treatment toxicity included a small but significant decline in albumin levels and increased total bilirubin; 1 experienced oesophagial varices bleeding (Grade III by CTCAE ver. 3) 3 months after the treated tumor and he recovered after endoscopic oesophagial ligation. All of other complications after treatment were Grade I (one dry cough and 2 radiation dermatitis). One and two-year actuarial data showed a 100\% local tumor control rate and an overall recurrence rate was $40 \%$, and all recurred tumors were diagnosed as out-field recurrence. Over-all survival rate was $100 \%$, and all of patients responded to treatment, and the therapy was well tolerated.

Conclusion(s): Proton-beam radiotherapy is an effective alternative treatment for locally unresectable hepatocellulae carcinoma not-feasible curative treatment in BCLC guideline. 


\section{PS10-014 \\ A Phase 2 Study of a Novel Transforming Growth Factor-Beta (TGF- $\beta$ ) Receptor I Kinase Inhibitor, LY2157299 Monohydrate, in Patients with Advanced Hepatocellular Carcinoma (HCC)}

Peter R. Galle ${ }^{1}$, Sandrine Faivre ${ }^{2}$, Armando Santoro ${ }^{3}$, Robin K. Kelley ${ }^{4}$, Philippe Merle ${ }^{5}$, Ed Gane $^{6}$, Jean-Yves Douillard ${ }^{7}$, Dirk Waldschmidt ${ }^{8}$, Mary Mulcahy ${ }^{9}$, Charlotte Costentin ${ }^{10}$, Beatriz Minguez ${ }^{11}$, Pasqua P. Papappicco ${ }^{12}$, Ivelina Gueorguieva ${ }^{13}$, Ann Cleverly ${ }^{14}$, Durisala Desaiah ${ }^{15}$, Michael M. Lahn ${ }^{16}$, Sophie Ameryckx ${ }^{17}$, Karim A. Benhadji ${ }^{16}$, Eric Raymond ${ }^{18}$, Gianluigi Giannelli ${ }^{12 *}$

${ }^{1}$ I Medizinische Klinik und Poliklinik, Universitätsmedizin, Berlin, Germany; ${ }^{2}$ Department of Service de Cancérologie, Hospital Beaujon, Clichy, France; ${ }^{3}$ Department of Oncology, Istituto Clinico Humanitas, Milano, Italy; ${ }^{4}$ Division of Hematology Oncology, Department of Medicine, University of California, San Francisco, United States; ${ }^{5}$ Molecular Physiopathology and New Therapies in Viral Hepatitis, Hospital De La Croix Rousse, Lyon, France; ${ }^{6}$ New Zealand Liver Transplant Unit, Auckland City Hospital, Auckland, New Zealand; ${ }^{7}$ Integrated Centers of Oncology, Centre Rene Gauducheau, Saint Herblain Cedex, France; ${ }^{8}$ Klinik I für Innere Medizin, Universitätsklinikum Köln, Köln, Germany; ${ }^{9}$ Medicine, Hematology Oncology Division; Feinberg School of Medicine, Northwestern University, Chicago, United States; ${ }^{10}$ Department of Hepatology, Hospital Henri Mondor, Creteil, France; ${ }^{11}$ Liver Unit, Medical Oncology, Vall d'Hebron Hospital, Barcelona, Spain; ${ }^{12}$ Internal Medicine, Immunology, Infectious Diseases, University of Bari Medical School, Bari, Italy; ${ }^{13} \mathrm{PK} / \mathrm{PD}$, Eli Lilly and Company, Indianapolis, United States; ${ }^{14}$ Statistics, Eli Lilly and Company, Indianapolis, United States; ${ }^{15}$ Global Scientific Communications, Eli Lilly and Company, Indianapolis, United States; ${ }^{16}$ Medical, Eli Lilly and Company, Indianapolis, United States; ${ }^{17}$ Clinical Operations, Eli Lilly and Company, Indianapolis, United States; ${ }^{18}$ Department of Medical Oncology, Hospital Beaujon, Clichy, France

Introduction: TGF- $\beta$ signaling is associated with HCC progression in moderate to poorly differentiated tumors overexpressing alpha-fetoprotein (AFP) levels. We report here the safety, antitumor activity and overall survival (OS) in HCC patients with elevated AFP treated with LY.

Methods: Patients with advanced HCC who progressed on sorafenib (SF) or are ineligible to receive SF, advanced ChildPugh A/B7 HCC, AFP $\geq 1.5 x$ ULN, ECOG PS $\leq 1$, measurable disease (RECIST 1.1), and $\leq 1$ prior systemic regimen were eligible. LY was administered as intermittent dosing of 14 days on/off (28 days $=1$ cycle). Patients were randomized to either
$160 \mathrm{mg} /$ day (Arm A) or $300 \mathrm{mg} /$ day (Arm B) LY. Primary endpoints were time-to-progression (TTP) and biomarker changes (serum AFP, TGF- $\beta$ and E-cadherin) for each dose. AFP responders were defined as $\geq 20 \%$ decline from baseline. Secondary endpoints included toxicity (CTCAE, V 4.0) and pharmacokinetics (PK). Available data from interims are presented.

Results: 109 patients were enrolled (Arm $A=37 ; B=72$ ). Baseline characteristics were (Arm A/B): median age 61/63 years; PS = 0, 60\%/53\%; Child-Pugh A 97\%/88\%; etiology: hepatitis C 30\%/38\%, hepatitis B 27\%/24\%, alcohol $24 \% / 25 \%$. Overall, $78 \% / 83 \%$ of patients had received prior SF; $65 \% / 57 \%$ had AFP $\geq 400 \mathrm{ng} / \mathrm{mL}$. Median TTP was 12.0 weeks $(90 \%$ CI: $6.6,12.6)$ in the overall population (Arm A/B, $12.1 / 10.0$ weeks). In SF-naïve patients, TTP was 18.3 weeks (90\% CI: 6.6-42.4). TTP was higher in the non-alcohol compared to alcohol-only etiology group (median 12.1 vs. 6.1 weeks). Median baseline serum TGF- $\beta 1$ was $3.4 \mathrm{ng} / \mathrm{mL}$ (range: 1.4-3.7) and E-cadherin was $6.1 \mathrm{mg} / \mathrm{mL}$ (range: $1.9-17.3$ ). AFP decline of $\geq 20 \%$ occurred in $24 \%$ of patients. Based on the latest interim, median OS was 36 weeks. In AFP responders, median OS was 93.1 weeks vs 29.6 weeks in non AFP responders ( $\log$ Rank $\mathrm{p}=0.0006)$. Four patients discontinued treatment due to AEs. Most common grade 3/4 related AEs were: neutropenia $(n=3)$, GI bleeding $(n=2)$, fatigue $(n=2)$, and anemia $(n=2)$. Preliminary PK analysis $(n=51)$ demonstrated moderate interpatient exposure variability (42\%).

Conclusion(s): Based on the manageable toxicity profile, the evidence for biomarker/TTP responses, analysis of the aggregate PK/PD data, and the OS data, the $300 \mathrm{mg} /$ day dose was chosen for future studies in HCC.

\section{PS10-017 \\ Survival Advantage with the Use of Metformin in Hepatocellular Carcinoma Patients Receiving Radiotherapy: A Propensity Score Matching Analysis Won Il Jang ${ }^{1}$, Mi-Sook Kim ${ }^{1 *}$, Jung Sub Lim², Chul Koo Cho', Hyung Jun Yoo', Young Seok Seo', Chul Ju Han', Su Cheol Park ${ }^{3}$, Chul Seung Kay', Myungsoo Kim ${ }^{4}$, Hong Seok Jang ${ }^{4}$, Dong Soo Lee ${ }^{4}$, Ah Ram Chang ${ }^{5}$, Hae Jin Park ${ }^{5}$ \\ ${ }^{1}$ Radiation Oncology, Korea Institute of Radiological \& Medical Sciences, Seoul, Korea; ${ }^{2}$ Pediatrics, Korea Institute of Radiological \& Medical Sciences, Seoul, Korea; ${ }^{3}$ Internal Medicine, Korea Institute of Radiological \& Medical Sciences, Seoul, Korea; ${ }^{4}$ Radiation Oncology, College of Medicine, The Catholic University of Korea, Seoul, Korea; ${ }^{5}$ Radiation Oncology, Soonchunhyang University Hospital, Seoul, Korea}

Introduction: Metformin use has been associated with a decreased risk of cancer and mortality. However, its effects on the survival of hepatocellular carcinoma (HCC) patients are 


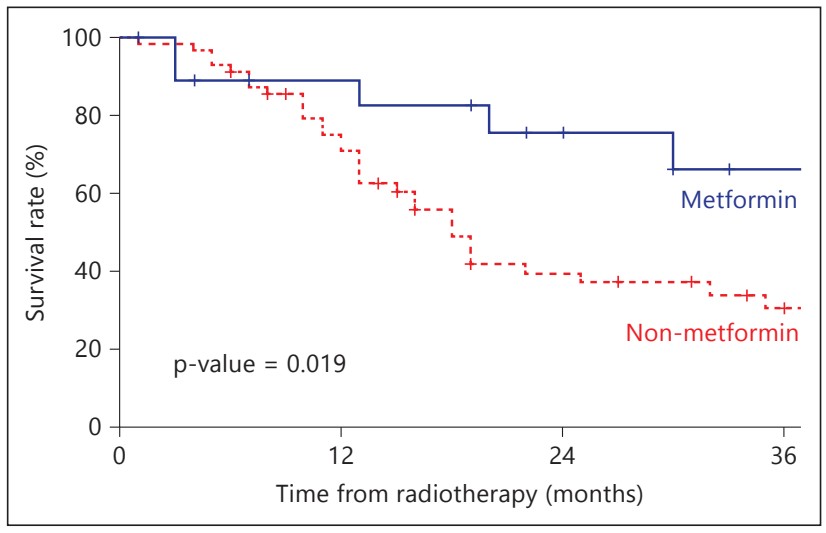

Fig. 1. Overall survival in the metformin group than non-metformin group after propensity score matching (for abstract PS10-017).

not defined. We perfomred this study to evaluate effects on the clinical outcomes of HCC patients received radiotherapy.

Methods: The medical records of 217 HCC patients treated with stereotactic body or hypofractionated radiotherapy. Of the enrolled patients, 48 had type 2 diabetes and 19 used metformin. We performed a 1:3 case-control study comparing patients who received metformin (metformin group) and those who did not (non-metformin group) using propensity score matching.

Results: In the propensity score-matched cohort ( $\mathrm{n}=$ 76), the overall survival (OS), progression-free survival (PFS), and extrahepatic metastasis-free survival (EMFS) rates were higher in the metformin group than non-metformin group ( 2 years, $76 \%$ vs. $39 \%, p=0.019 ; 46 \%$ vs. $19 \%$, $p=0.016$; and $85 \%$ vs. $80 \%, p=0.044$, respectively). Multivariate analysis revealed that metformin use (adjusted hazard ratio [HR], 2.933; 95\% confidence interval [CI], 1.125-7.634; $p=0.028$ ) and tumor size of more than $5 \mathrm{~cm}$ (HR, 2.319; 95\% CI, 1.2084.453; $\mathrm{p}=0.011$ ) were significant factors for OS.

Conclusion(s): Metformin use in HCC patients receiving radiotherapy was associated with higher OS, PFS, and EMFS rates. Therefore, metformin might be potentially useful in the combined treatment of inoperable HCC.

\section{PS10-018 \\ Partial Splenic Embolization for Secondary Hypersplenism in Hepatocellular Carcinoma with Cirrhosis}

\author{
Dae Sung Lee Hong Joo Kim, Yong Kyun Cho, \\ Woo Kyu Jeon, Byung Ik Kim* \\ Internal Medicine, Sungkyunkwan University School of \\ Medicine, Kangbuk Samsung Hospital, Seoul, Korea
}

Introduction: Patients with hepatocellular carcinoma (HCC) and cirrhosis have a tendency of bleeding due to low platelet level. The bleeding risk in HCC patients with cirrhosis makes invasive therapy difficult in operation, paracentesis, trans-arterial chemo-embolization (TACE) and radiofrequency ablation (RFA). Partial splenic embolization (PSE) has been used for treatment of thrombocytopenia caused by secondary hypersplenism in patients with liver cirrhosis. The authors investigated the effect and safety of PSE in HCC with cirrhosis.

Methods: Thirteen HCC with cirrhotic patients were presented to our institution between March 2001 and December 2011 with thrombocytopenia (platelet count $<45 \times 1000 / \mathrm{mm} 3$ ) with hypersplenism and the data was collected retrospectively. Pre-, and post-infarcted splenic volume was measured by computed tomography. The changes of platelet count after PSE were evaluated by repeated measures ANOVA. The result of WBC, hemoglobin (Hb), AST, ALT, albumin, bilirubin, INR, Child-Turcotte-Pugh (CTP) score, alpha fetoprotein (AFP), proteins induced by vitamin K antagonist-II (PIVKA-II) and liver volume were analyzed by General linear model after PSE. In addition, the state of HCC with cirrhosis after PSE was analyzed for the aggravation and side effect.

Results: PSE was performed in all 13 patients in this study. Five patients were female (38.4\%) and mean age was $50 \pm 17$ years old. The mean infarction ratio was $60.6 \% \pm 16.5 \%$ (range $32.5 \%-95.3 \%$ ). The platelet count significantly increased from $(34.3 \pm 6.9) \times 103 / \mathrm{mm} 3$ before PSE to $(119.7 \pm 52.2) \times 1000 / \mathrm{mm} 3$ and $(97.4 \pm 68.5) \times 1000 / \mathrm{mm} 3$ at 1 month and 6 months after PSE, respectively $(p=0.001)$. The splenic volume is significantly decreased after PSE $(p<0.001)$. $\mathrm{Hb}, \mathrm{AST}, \mathrm{ALT}$, albumin, bilirubin, INR, CTP score, AFP and PiVKA-II were not changed significantly after PSE (respectively, p-value is $0.391,0.638,0.134,0.278,0.609,0.898$, $0.932,0.357$ and 0.938$)$. However, WBC level was significantly increased from $2731 \pm 1112 / \mathrm{mm} 3$ before PSE to $5936 \pm 3580$ / $\mathrm{mm} 3$ and $4942 \pm 1848 / \mathrm{mm} 3$, respectively at one and six month later $(\mathrm{p}=0.004)$. There was one case of newly developed HCC after PSE ( $n=1,7.6 \%)$ without aggravation of previous HCC with cirrhosis. There were minor complications which were controlled easily; pleural effusion $(n=4,30.7 \%)$, ascites $(n=$ $2,15.3 \%)$, atelectasis ( $n=1,7.6 \%)$.

Conclusion(s): PSE is an effective procedure for the treatment of thrombocytopenia by secondary hypersplenism 
in HCC patients with cirrhosis. There were no severe side effects including aggravation of HCC with cirrhosis after PSE. The infarction ratio and WBC count are important factors for the platelet count increase after PSE in HCC with cirrhosis.

\section{PS10-019 \\ Impact of Single Nucleotide Polymorphisms (SNPS) of Drug-Metabolizing Genes on the Therapeutic Efficacy of GEMOX Alone or with Cetuximab in Advanced Biliary Tract Cancers}

\author{
Nai-Jung Chiang ${ }^{1}$, Jeffrey S. Chang ${ }^{1}$, Chiun $\mathrm{Hsu}^{2}$, \\ Jen-Shi Chen ${ }^{3}$, Yee Chao ${ }^{4}$, Yang-Shen Shan ${ }^{5}$, \\ Shiu-Feng Huang ${ }^{6}$, Li-Tzong Chen ${ }^{1 *}$ \\ ${ }^{1}$ National Institute of Cancer Research, National Health \\ Research Institutes, Tainan, Taiwan; ${ }^{2}$ Department \\ of Oncology, National Taiwan University Hospital, \\ Taipei, Taiwan; ${ }^{3}$ Division of Hematology/Oncology, \\ Department of Internal Medicine, Linkou Chang Gung \\ Memorial Hospital, Taoyuan, Taiwan; ${ }^{4}$ Taipei Veterans \\ General Hospital, Cancer Center, Taipei, Taiwan; \\ ${ }^{5}$ National Cheng Kung University Hospital, Department \\ of Surgery, Tainan, Taiwan; ${ }^{6}$ Institute of Molecular and \\ Genomic Medicine, National Health Research Institutes, \\ Miaoli, Taiwan
}

Introduction: T1210 was a randomized phase II trial that showed adding cetuximab could marginally improve the overall response rate (ORR) and median progression-free survival (PFS) of advanced biliary tract cancer (ABTC) patients receiving GEMOX. The aim of this study is to evaluate the clinical relevance of drug metabolism-related SNPs in an uniform cohort.

Methods: DNA was extracted from prospectively collected peripheral blood mononuclear cells (PBMC) of 122 participants in T1210 study, and analyzed by mass spectrometry-based genotyping (Sequenom, San Diego, CA, USA), including 11 SNPs for RRM1, DCK and hENT1 (for gemcitabine), ERCC1, GSTP1 and MGMT (for oxaliplatin) and EGFR and FCGR3A (for cetuximab). Those clinically relevant SNPs were then validated by direct sequencing.

Results: Of the 11 SNPs of 8 drug-metabolizing genes, four (GSTP1 rs1695, DCK rs4694362, RRM1 rs 12806698 and RRM1 rs 11030918) showed prognostic significance for PFS by mass spectrometry-based genotyping. After direct sequencing, patients with homozygous wild-type of GSTP1 rs1695, RRM1 rs12806698 or RRM1 rs11030918 had better PFS than those with any variant allele of corresponding gene regardless of treatment arm. The median PFS was the best for C-GEMOX/wild-type SNP and subsequently followed by C-GEMOX/variant SNP, GEMOX/wild-type SNP and GEMOX/ variant SNP, for GSTP1 rs1695 ( $\mathrm{p}=0.2)$ ), RRM1 rs12806698 $(\mathrm{p}=0.05)$ and RRM1 rs11030918 ( $\mathrm{p}=0.049)$. For patients with wild-type of DCK rs4694362, C-GEMOX treatment resulted in longer PFS than GEMOX. In contrast, adding cetuximab did not benefit patients with any variant alleles.

Conclusion(s): C-GEMOX exhibited better PFS than GEMOX regardless of the genotypes of GSTP1 rs1695, RRM1 rs12806698 and RRM1 rs11030918. In contrast, C-GEMOX treatment is only suggested in patients with wild-type genotype of DCK rs4694362, indicating that DCK rs4694362 may be a potential biomarker for selecting patients to achieve significant survival benefit from adding cetuximab to chemotherapy.

\section{PS10-020 \\ Axitinib as Second-Line Treatment for Advanced Hepatocellular Carcinoma (HCC): Preliminary Results of A Phase 2 Trial \\ Zhong-Zhe Lin ${ }^{1}$, Yee Chao ${ }^{2}$, Ying-Chun Shen ${ }^{1}$, Bang-Bin Chen ${ }^{3}$, Chih-Hung Hsu', Yu-Yun Shao', Ying-Hui Lee ${ }^{1}$, Chiun Hsu ${ }^{1 *}$, Ann-Lii Cheng ${ }^{1}$ \\ ${ }^{1}$ Oncology, National Taiwan University Hospital, Taipei, Taiwan; ${ }^{2}$ Internal Medicine, Veteran General Hospital, Taipei, Taiwan; ${ }^{3}$ Medical Imaging, National Taiwan University Hospital, Taipei, Taiwan}

Introduction: Axitinib can inhibit the activity of multiple angiogenesis-related signaling pathways and can improve survival in patients with renal cell carcinoma after failure from sorafenib treatment. Its efficacy in HCC patients as second-line treatment was undefined.

Methods: Eligible criteria included histological or clinical diagnosis of HCC, documented progression with or intolerance to sorafenib treatment as first-line therapy for advanced HCC, ECOG score 0 or 1, Child-Pugh class A, and measurable tumors (by RECIST 1.1). All enrolled patients received axitinib $5 \mathrm{mg}$ orally twice daily until tumor progression or unacceptable toxicity. Dose titration was done according to severity of adverse events. Tumor response was assessed after 4 weeks and 8 weeks of treatment and every 8 weeks thereafter until objective disease progression. The primary endpoint was disease stabilization (DS), defined as complete or partial response according to RECIST or stable disease that lasted for at least 8 weeks without progression of tumor related symptoms.

Results: Since April 2011, 28 patients were enrolled (M: F 23:5; median age 58.6 years (range 38.8-76.2); HBV/HCV 22/6; median alpha-fetoprotein (AFP) $723.2 \mathrm{ng} / \mathrm{mL}$ (range 3.1-50563.4); CLIP score 0-1/2/3/4 10/11/6/1; extrahepatic metastases 21 patients). All patients had documented progression after prior sorafenib treatment. The DS rate in 24 evaluable patients was 0.58 (95\% C.I. 0.37-0.82). Partial response was documented in 2 patients. As of 10 April 2014 the median progression-free survival and overall survival was 23.6 weeks (95\% C.I. 8.5-38.6) and 43.1 weeks (95\% C.I. 10.8-75.4), respectively. Grade 3/4 adverse events occurring 
to $>10 \%$ of patients included transaminase elevation (5 patients), bleeding ( 4 patients), and hand-foot skin reaction (3 patients).

Conclusion(s): Axitinib can achieve DS in a proportion of advanced HCC patients after failure from sorafenib treatment with manageable safety profile.

\section{PS10-021 \\ Metronomic Chemotherapy Compared with Conventional Transcatheter Arterial Chemoembolization in Patients with Advanced Hepatocellular Carcinoma with Poor Liver Function}

Hyun Yang ${ }^{1}$, Hee Chul Nam ${ }^{1}$, Hae Lim Lee ${ }^{1}$, Sung Won Lee ${ }^{1}$, Do Seon Song ${ }^{1}$, Jeong Suk Oh², Jeong Won Jang ${ }^{1}$, Ho Jong Chun ${ }^{2}$, Young Seok Kim³, Si Hyun Bae ${ }^{1 *}$, Jong Young Choi ${ }^{1}$, Seung Kew Yoon ${ }^{1}$

${ }^{1}$ Department of Internal Medicine, Catholic Liver Research Center, The Catholic University of Korea, Seoul, Korea, Seoul, Korea; ${ }^{2}$ Department of Radiology, College of Medicine, The Catholic University of Korea, Seoul, Korea, Seoul, Korea; ${ }^{3}$ Department of Internal Medicine, Soon Chun Hyang University College of Medicine, Seoul, Korea

Introduction: Metronomic chemotherapy (MET) is a treatment modality characterized by frequent administration of comparatively low doses of chemotherapeutic agent without extended break.

The aim of this study is to evaluate the efficacy and safety of MET compared with conventional transcatheter arterial chemoembolization (TACE) in patients with advanced hepatocellular carcinoma (HCC) and poor liver function.

Methods: Major inclusion criteria were Barcelona Clinic Liver Cancer stage C and child-pugh class B or C. Minor criteria were the presence of portal vein tumor thrombosis (PVTT), largest tumor diameter $(>8 \mathrm{~cm})$ and infiltrative type HCC. We analyzed patients who fulfilled all major criteria and at least one minor criteria. Total of 45 patients who had undergone MET and 30 patients who had received conventional TACE between April, 2007 and August, 2013 were retrospectively analyzed. Epirubicin $35 \mathrm{mg} /$ body surface area (BSA) was administrated every 4 weeks, and cisplatin $15 \mathrm{mg} / \mathrm{BSA}$ and 5-fluorouracil $50 \mathrm{mg} / \mathrm{BSA}$ were administrated weekly for 3 weeks with one week break between each cycle. In the MET group, the chemotherapeutic agents were administered through hepatic arterial chemoport.

Results: Median follow up period was 97 days (4-663). The patients in the MET group received median 2 cycles (range 1-6) of chemotherapy and the patients in the TACE group, 1 cycle (range 1-4) respectively. Overall survival time of the MET group and the TACE group were 130 days (4-663) and 89 days (7-367), respectively $(\mathrm{P}>0.05)$.
Multivariate analysis revealed extrahepatic metastasis (hazard ratio $(\mathrm{HR})=1.832, \mathrm{P}=0.043)$, larger tumor size $(\mathrm{HR}=$ $1.066, P=0.022)$, and TACE $(H R=1.891, P=0.029)$ as independent predictive factors of poorer survival.

Conclusion(s): MET showed relatively a favorable outcome in terms of overall survival than conventional TACE in advanced HCC with poor liver function. Therefore, in cases of PVTT, large tumor, infiltrative type HCC and poor liver function, MET may be considered as a treatment option.

\section{PS10-022 \\ High Seroprevalence of Human Herpesvirus Type 8 in Hepatoma Patients with Decompensated Cirrhosis \\ Cheng-Chuan Su ${ }^{1 *}$,Kuo-Chih Tseng ${ }^{2}$, Ming-Nan Lin ${ }^{3}$, Tsung-Cheng Hsieh ${ }^{4}$, Tang-Yuan $\mathrm{Chu}^{4}$ \\ ${ }^{1}$ Departments of Clinical Pathology, Buddhist Dalin Tzu Chi Hospital, Chiayi, Taiwan; ${ }^{2}$ Department of Internal Medicine, Buddhist Dalin, Chiayi, Taiwan; ${ }^{3}$ Department of Family Medicine, Buddhist Dalin Tzu Chi Hospital, Chiayi, Taiwan; ${ }^{4}$ Institute of Medical Sciences, Tzu Chi University, Hualien, Taiwan}

Introduction: Immunologic insufficiency and a high seroprevalence of human herpesvirus type 8 (HHV-8) have been found in cirrhotics. Nearly all hepatocellular carcinoma (HCC) patients are cirrhotic and have immunoabnormalities. This study assessed the HHV-8 seroprevalence and hemograms in HCC patients.

Methods: Blood samples from 95 HCC patients; 95 age-, sex-, and Child-Pugh class-matched cirrhosis patients; and 95 age- and sex-matched healthy controls were analyzed for antiHHV-8 antibody; HHV-8 DNA; and lymphocyte, monocyte, and platelet counts.

Results: Similar to cirrhotics, HCC patients had lower lymphocyte and platelet counts and a higher monocyte count than the healthy controls (each $\mathrm{p}<0.0001$ ). HCC patients, and particularly those with a severe Child-Pugh class, had higher platelet counts than the corresponding cirrhotics $(p=0.003$ and 0.002 , respectively). HHV-8 seropositivity and antibody titers in HCC patients were comparable with values in cirrhotics and were much higher than in controls (both $\mathrm{p}<0.0001$ ). HCC patients, but not cirrhotics, had a higher prevalence of high anti-HHV-8 antibody titers $(\geq 1: 160)$ than did healthy controls ( $p=0003$ ). HCC patients with lymphopenia or thrombocytopenia had lower HHV-8 seropositivity than patients without ( $p=0.04$ and 0.01 , respectively). One HCC patient and one cirrhosis patient were positive for HHV-8 DNA.

Conclusion(s): HCC patients seemed to suffer from less severe or shorter-duration of portal hypertension compared with the Child-Pugh class-matched cirrhotics. HCC patients had a high HHV-8 seroprevalence, which seemed to be inversely associated with lymphopenia and thrombocytopenia. 
PS10-023

\section{Survival Benefit of Particle Radiotherapy Using Protons and Carbon-Ions as First- Line Treatment for Initial Hepatocellular Carcinoma}

Kazuki Terashima*, Yusuke Demizu, Fumiko Nagano, Kuniaki Katsui, Masaru Takagi, Dongcun Jin, Naoki Hashimoto, Masayuki Mima, Osamu Fujii, Tomoaki Okimoto, Nobukazu Fuwa

Department of Radiology, Hyogo Ion Beam Medical Center, Tatsuno, Japan

Introduction: The high efficacy of particle radiotherapy (PRT) in patients with hepatocellular carcinoma (HCC) has been indicated by several reports. In this study, we retrospectively analyzed the survival benefit of first-line PRT for initial HCC to compare to surgical resection and radiofrequency ablation.

Methods: A total of 148 tumors from 140 patients from April 2001 to March 2009 were treated with PRT using proton or carbon-ion beams as first-line treatment for initial HCC.

Results: The median follow-up period was 30 months. The local control, overall survival (OS), and disease-free survival rates at 5-year for all 140 patients were 87.0, 58.3, and $11.0 \%$, respectively (Fig. 1 ). The OS rates at 2-, 3-, and 4-year for tumor size $<30 \mathrm{~mm}$ were $87.6,77.8$, and $71.6 \%$, respectively. The 5-year OS rate for operable stage according to the Barcelona Clinic Liver Cancer classification was $66.7 \%$, and the OS rate at 5-year for inoperable stage was $46.7 \%$. Acute and late adverse events were feasible and required no invasive treatment.

Conclusion(s): The OS of PRT with less invasion for small HCC (tumor size $<30 \mathrm{~mm}$ ) and operable HCC were comparable to radiofrequency ablation and surgical resection,

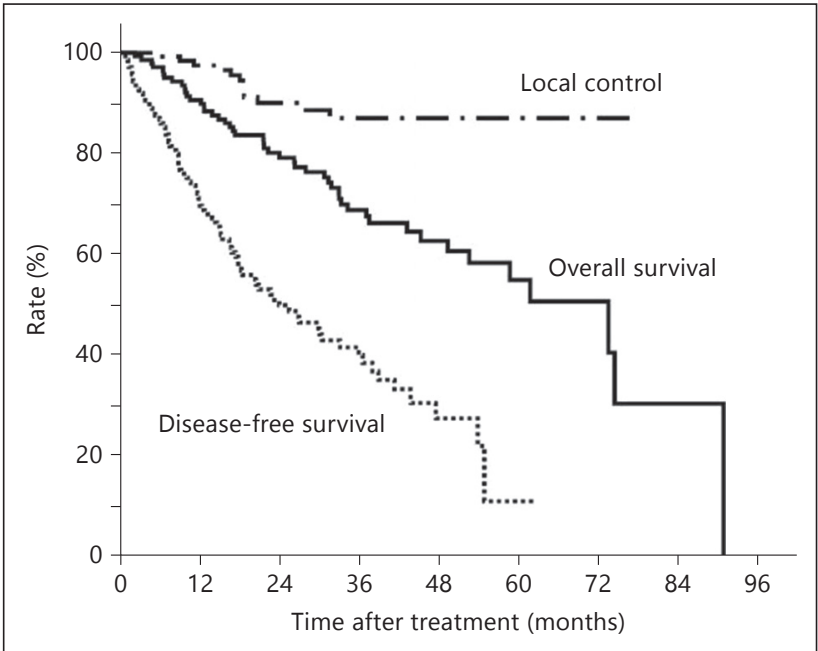

Fig. 1. Local control, disease-free survival, and overall survival rates for all 140 patients (for abstract PS10-023). respectively, generally considered as curative treatment. PRT has the potential to be used as a major treatment option for HCC.

\section{PS10-024}

Cannabinoid Receptor 1 Promotes Hepatic Lipid Accumulation and Lipotoxicity through the Induction of SREBP-1c Expression in Zebrafish

Chia-Chun Hsu ${ }^{1}$, Guor Mour Her ${ }^{2 *}$

${ }^{1}$ Radiology, Taichung Tzu Chi Hospital, Taichung, Taiwan; ${ }^{2}$ Institute of Bioscience and Biotechnology, National Taiwan Ocean University, Keelung, Taiwan

Introduction: The activated cannabinoid receptor 1 (CB1R) is exclusively responsible for food intake and weight gain and regulates several pathological features associated with obesity in mammals. However, the precise role of CB1R in non-mammalian model systems is poorly understood.

Methods: To investigate the functions of CB1R in zebrafish liver, we conditionally expressed CB1R proteins using a liverspecific Tetoff transgenic system.

Results: In this study, we found hepatic lipid accumulation in CB1R transgenic zebrafish (CB) without doxycycline treatment (-Dox) and a suppression of CB1R expression, resulting in the loss of lipid accumulation in the livers of $\mathrm{CB}$ fish that received doxycycline treatment (?Dox). Oil Red 0 (ORO)-stained hepatocytes were predominant in the liver buds of CB-Dox larvae, indicating that CB1R functionally promotes lipid accumulation during $\mathrm{CB}$ hepatogenesis. More than $73 \%$ of CB-Dox adults showed increased lipid content, which leads, in turn, to steatosis. Liver histology and ORO staining of CB-Dox hepatocytes also indicated the accumulation of fatty droplets in the CB liver samples, consistent with the specific pathological features of liver steatosis or steatohepatitis. We also found that hepatic CB1R overexpression accompanies the stimulation of the lipogenic transcription factor SREBP-1c and its target enzymes, acetyl coenzyme-A carboxylase-1 (ACC1) and fatty acid synthase (FAS), and increases de novo fatty acid synthesis.

Conclusion(s): This study is the first to report CB1R as a potential hepatic stimulator for zebrafish liver steatosis. 


\section{PS10-025}

\section{Synergistic Effects of Metformin in} Combination with Rapamycin on Hepatocellular Carcinoma Cell Lines

Kwang-Woong Lee*, Suk-Won Suh, Jaehong Jeong, Hyeyoung Kim, Nam-Joon Yi, Kyung-Suk Suh

Department of Surgery, Seoul National University College of Medicine, Seoul, Korea

Introduction: Several studies have shown that metformin had an anti-tumor effect on several cancers, including hepatocellular carcinoma (HCC). After liver transplantation (LT), immunosuppression is needed to avoid rejection and graft loss, however, it can stimulate HCC recurrence and progression. The aim of this study was to evaluate interactions between metformin and immunosuppressive agents for antitumor activity.

Methods: Antiproliferative effects were assessed using HCC cell line as HepG2, Hep3B, Huh7. Metformin (10 mM/L) and several immunosuppressive agents such as Sirolimus ( $5 \mathrm{ng} / \mathrm{ml}$ ), Tacrolimus (5 ng/ml), and MMF (500 ng/ml) were evaluated. Cell viability was determined using a standard colorimetric 3-(4,5-dimethylthiazol-2-yl)-2 5-diphenyltetrazolium bromide (MTT) assay after 48 hours. Western blot analysis were performed to investigate protein levels in HCC cells.

Results: Metformin and Sirolimus itself had antitumor effect in all of the HCC cell lines (73\% and 66\%, HepG2; 84\% and $73 \%$, Hep3B; 77\% and 66\%, Huh7). They also had significant synergistic antiproliferative effect in all of the HCC cell lines (68\%, HepG2; 57\%, Hep3B; 63\%, Huh7), however, there was no synergistic effect with other immunosuppressive agents. In addition, combination of metformin, sirolimus and MMF showed some synergistic antiproliferative effect in Hep3B and Huh7 HCC cell lines (53\%, Hep3b; 53\%, HuH7).
Conclusion(s): Metformin had synergistic interactions with Sirolimus in terms of antitumor effects for HCC cell lines. These results may provide a foundation for further studies to evaluate combination therapies for patients with HCC who underwent LT in clinical era.

\section{PS10-026 \\ Clinical Outcomes of Cyberknife Stereotactic Body Radiation Therapy for Unresectable Hepatocellular Carcinoma

\author{
Jenny Que*, Li-Ching Lin, Chia-Hui Lin, Kuei-Li Lin, \\ Yu-Wei Lin, Ching-Chieh Yang \\ Radiation Oncology, Chi Mei Medical Center, Tainan, \\ Taiwan
}

Introduction: Stereotactic body radiation therapy (SBRT) has been an emerging non-invasive local treatment option for patients with hepatocellular carcinoma (HCC) when established curative treatment modalities cannot be applied. In the present study, we report our clinical experience regarding Cyberknife SBRT for unresectable HCC and evaluate the efficacy and clinical outcomes of this highly sophisticated treatment modality.

Methods: Between 2008 and 2012, 115 patients with unresectable HCC treated with Cyberknife SBRT were retrospectively analyzed. Dose range from 26 Gy to 40 Gy in 3 to 5 fractions were given in 3 to 5 consecutive days. The tumor response was determined by CT or MRI performed 2-3 months after the completion of SBRT. Tumor response was assessed according to the Response Evaluation Criteria in Solid Tumor criteria (RECIST). The probability of cumulative survival was calculated according to the Kaplan-Meier method and compared statistically using log-rank test. Univariate and

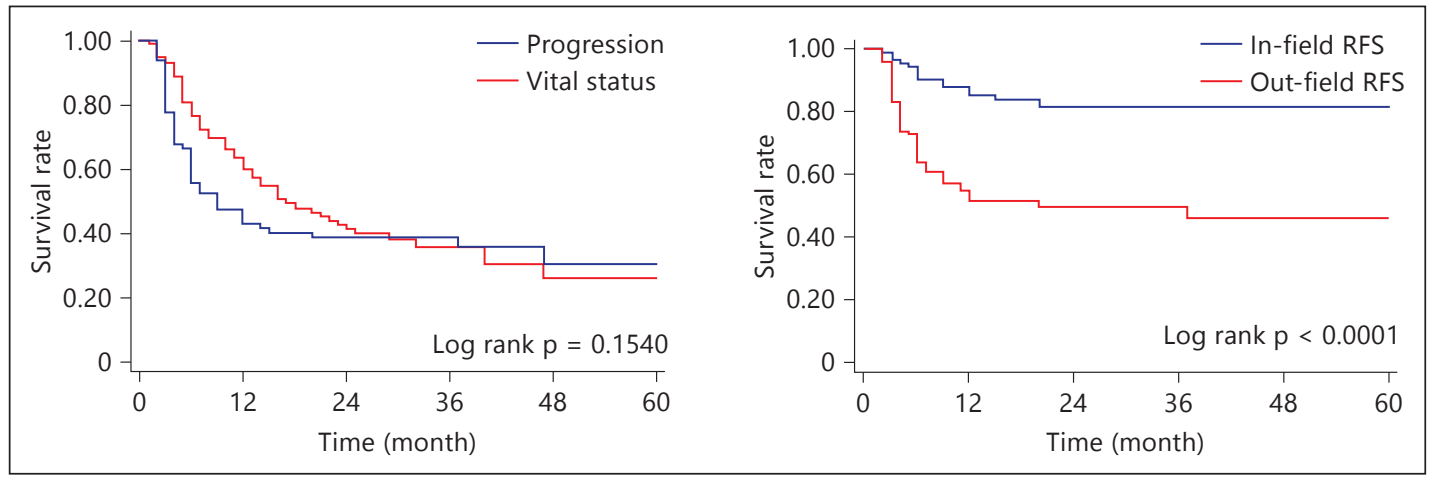

Fig. 1. Overall survival curve of 115 patients treated with Cyberknife SBRT. 1-yr and 2-yrs OSR were 63.5\% (54-71.5\%) and 41.3\% (31.6-50.6\%), median survival times was 15 months (4-25 mos.), While 1-yr and 2-yrs Progression-free survival rate were 42.8\% (33.0-52.2\%) and 38.8\% (29.0-48.4\%), median progressive-free survival times was 6 months, respectively (for abstract PS10-026). 
multivariate analysis were performed using Cox porportional hazard models.

Results: The median follow-up was 15.5 months (range, 2-60 months). We found that $48.7 \%$ patients achieved a complete response and $40 \%$ achieved a partial response. Median survival was 15 months (4-25 mos.) months. Overall survival (OS) at 1-, 2- and 3-years were 63.5\% (54-71.5\%), $41.3 \%$ (31.6-50.6\%) and 35.8\% (25.8-45.9\%). While 1- and 2-years Progression-free Survival (PFS) were 42.8\% (33.0$52.2 \%)$ and $38.8 \%$ (29.0-48.4\%), median to progression was 6 months. In-field intrahepatic recurrence free rates was $85.3 \%(76.2-91.1 \%)$ and $81.6 \%(72.2-88.6 \%)$ at $1-y r$ and 2 yrs., median times was 13 months (6-25 mos.), respectively. While the 1-yr and 2-yrs Out-field intrahepatic-recurrence free rate were $52.5 \%$ (41.2-60.8\%) and 49.5\% (38.9-59.2\%), median times was 7 months (4-17 mos.), respectively. Multivariate analysis reveal Child-Pugh score (A vs B), Portal vein tumor thrombosis (PVTT( + ) vs PVTT(-)), Tumor size $(\leq 4 \mathrm{~cm}$ vs $>4-9 \mathrm{~cm} / \geq 10 \mathrm{~cm}$ ), Tumor response after SBRT (CR vs PR/ stable) were independent predictors of OS. Acute toxicities were mostly transient and tolerable.

Conclusion(s): SBRT appears to be an effective noninvasive treatment modality for the local management of unresectable HCC. These results suggested that SBRT can be a good alternative modality for the treatment of unresectable HCC unsuitable for other standard treatment. However, outfield intrahepatic recurrence remain a major cause of failure. Prospective studies of combinations of SBRT with other treatment modalities may be needed. 